
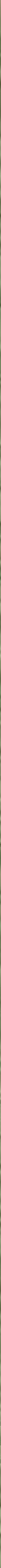

L 6 r r ver

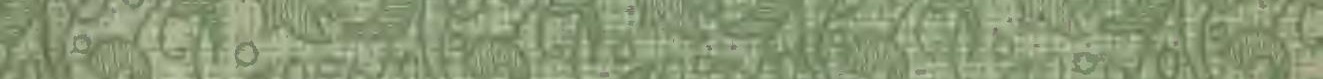

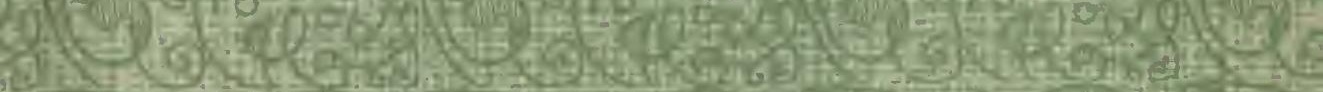

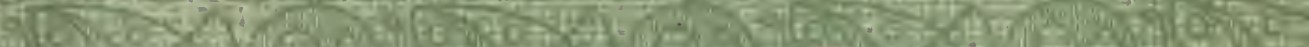

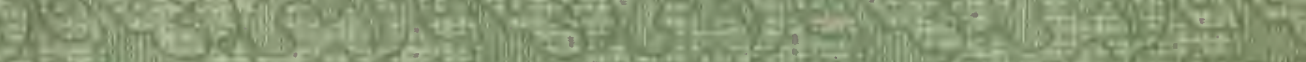
If

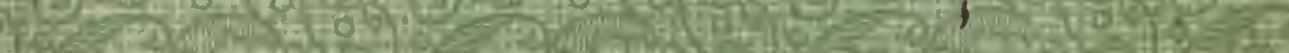

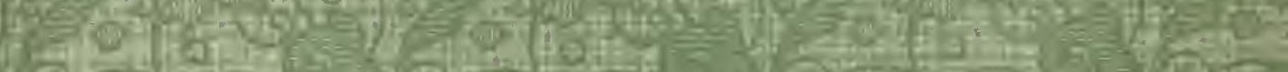
6.3. 19.

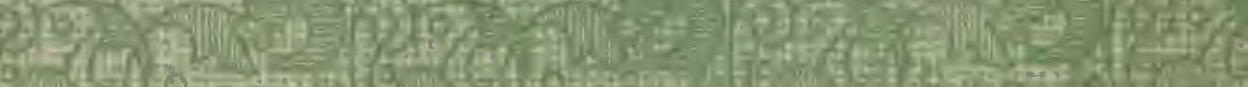

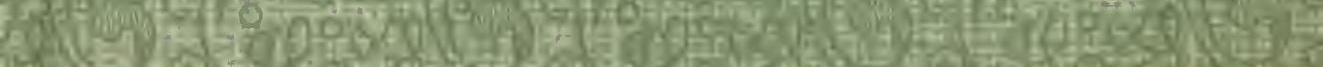
d. 39 . 

Digitized by the Internet Archive in 2008 with funding from Microsoft Corporation 



\section{H E M I S T R Y}

IN ITS APPLICATION TO

\section{AGRICULTURE AND PHYSIOLOGY.}

BY

!

JUSTUS LIEBIG, M.D.; Pн.D., F.R.S., M.R.I.A.,

PROFESSOR OF CHEMISTRY IN THE UNIVERSITY OF GIESSEN, ErC., ErC., ETC.

EDITED FROM THE MANUSCRIPT OF THE AUTHOR

By LYON PLAYFAIR, Pн.D. काTि ? $?$

VERY NUMIEROUS ADDITIONS, AND A NEW CHAPTER ON SOILS.

THIRD AMERICAN, FROM THE SECOND ENGLISH EDITION,

WITH

NOTES, AND APPENDIX,

BY

JOHN W. WEBSTER, M. D.,

ERVING PROFESSOR OF CHEMISTRY IN HARVARD UNIVERSITY.

012 2este

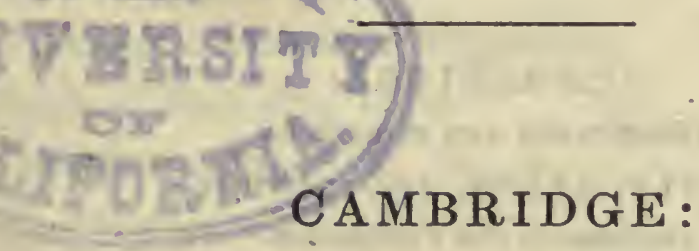

PUBLISHED BY JOHN OWEN.

BOSTON, JAMES MUNROE AND COMPANY, AND CHARLES C. LTTTLE AND JAMES BROWN;

NEW YORK, WILEY AND PUTNAM, AND GEORGE C. THURBURN; PHLADELPHIA, THOMAS, COWPERTHWAIT, AND COMPANY, AND CAREY AND HART;

BALTIMORE, CUSHINO AND BROTHER. 


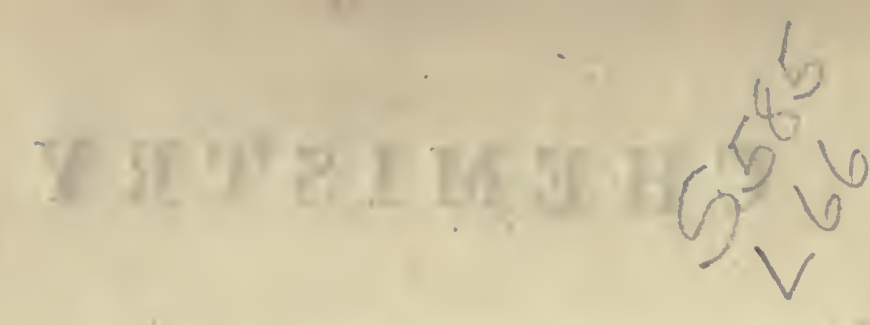

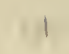

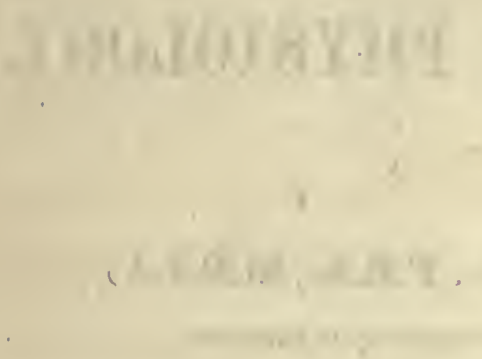

Entered according to Act of Congress, in the year 1842, by

J O H N OW N,

in the Clerk's office of the District Court of the District of Massachusetts.

58114

\section{A M B R I D G E ;}

STEREOTYPED AND PRINTED BY

METCALF, KEITH, AND NICHOLS, PRINTERS TO THE UNIVERSITY. 


\section{CONTENTS.}

PAGB

Preface to the Third American Edition . • v Dedication

Preface to the Second English Edition

- XIII OBJECT OF THE WORK XVII

21

\section{PÁRT FIRST.}

ON THE CHEMICAL PROCESSES IN THE NUTRITION OF VEGETABLES. CHAPTER

PAGE

I. - On the Constituent Elements of Plants . . - 24

II. - On the Assimilation of Carbon . . . 30

III. - On the Origin and Action of Humus . $\quad 63$

IV. - On the Assimilation of Hydrogen . . . 80

V. - On the Origin and Assimilation of Nitrogen . $\quad 85$

VI. - On the Inorganic Constituents of Plants : $\quad$ - 105

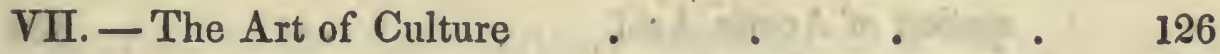

VIII. - On the Alternation (Rotation) of Crops $\quad \cdots 161$

IX. - On Manure . . . . . . . 174

Supplementary Chapter. - On the Chemical Constituents of Soils . . $\quad . \quad \ldots \quad 208$

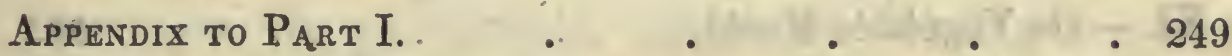

Action of Charcoal on Vegetation $\quad$ - 249

Mode of Manuring Vines . . . . 253

Root Secretions . . . . . . 256

Peat Compost .. . . . . . 258

Source of the Carbon of Plants : . . 260

Source of the Hydrogen of Plants . • . 263

Dependence of the Nutritive Qualities of Plants on 
Difference in the Power of Plants to decompose Ammonia

Practical Inferences

Use of Phosphate of Soda

Daniell's Artificial Manure

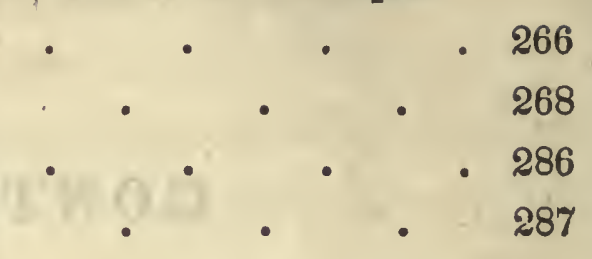

\section{PART SECOND.}

ON THE CHEIMICAL PROCESSES OF FERMENTATION, DECAY, AND PUTREFACTION.

CHAPTER PAGE

I. - Chemical Transformations 289

II. - On the Causes which effect Fermentation, Decay, and Putrefaction

III. - Fermentation and Putrefaction

IV. - On the Transformation of Bodies which do not contain Nitrogen as a constituent, and of those in which it is present

V. - Fermentation of Sugar

VI. - Eremacausis, or Decay 322

VII. - Eremacausis of Bodies destitute of Nitrogen : Formation of Acetic Acid

VIII. - Eremacausis of Substances containing Nitrogen : Nitrification 334

IX. - On Vinous Fermentation : Wine and Beer- - . 338

X. - On the Decay of Woody Fibre . • • 357

XI. - On Vegetable Mould . . $\quad$. $\quad$. 363

XII. - On the Mouldering of Bodies : Paper, Brown Coal, and Mineral Coal

XIII. - On Poisons, Contagions, and Miasms _ - 373 Appendix to Part II.

Tables, - Showing the Proportion between the Hessian and English Standard of Weights and Measures 


\section{PREFACE}

TO THE

\section{THIRD AMERICAN EDITION.}

This volume constitutes the First Part of Professor Liebig's Report on Organic Chemistry, drawn up by request of the British Association for the Advancement of Science.*

The interest excited in Great Britain on the appearance of this work from one of the most eminent chemists in Europe, and the high encomiums bestowed upon it by individuals, and learned bodies, together with the various notices of it which have been published by Professor Lindley, Professor Daubeny, and others, all concurring in the opinion, that the information it contains is of great amount, and that from its publication might be dated a new era

* The Second Part has just been published, viz., "Animal Chemistry, or Organic Chemistry in its Application to Physiology and Pathology. By Justus Liebig, M. D., F. R. S., M. R. I. A., Professor of Chemistry in the University of Giessen, \&c., \&c., \&c. Edited from the Author's Manuscript, by William Gregory, M. D., F. R. S., M. R. I. A., Professor of Medicine and Chemistry in the University and King's College, Aberdeen. With Additions, Notes, and Corrections, by Dr. Gregory, and others by John W. Webster, M. D., Erving Professor of Chemistry in Harvard University." 
in the art of agriculture, induced the editor to suggest its republication in this country.

Contrary to the expectations of the author, and of the editor, the work has received the attention not only of scientific readers, for whom it was written, but of practical agriculturists, and those who could hardly have been supposed prepared to derive much advantage from its perusal. The influence of the opinions of Professor Liebig, and the impetus the appearance of the present work gave to the advancement of scientific agriculture, have been evinced by the many publications which have since appeared, both in Great Britain and in this country.

What is valuable in too many of these publications, diluted as it has been and mingled with erroneous statements, was for the first time given in a consistent shape in the present work.

Although the fact that nitrogen is essential to the nutrition of plants was known before the publication of Professor Liebig's work, and it had, indeed, been ascertained by Saussure, that germinating seeds absorb nitrogen, it was not supposed that it is derived from the atmosphere exclusively. And this has been deemed the chief discovery_of the author, so far as practical questions are concerned. It had indeed been suspected, that very small quantities of ammonia in the atmosphere might furnish the nitrogen, ammonia being a compound of nitrogen and hydrogen. It may be objected, that the quantity of ammonia present in the atmosphere, and in rain and snow water, is 
exceedingly small, quite insufficient for the supply of all the nitrogen that enters into the vegetable structure. To this it has been replied by Professor Lindley, in an elaborate review of Liebig's work, that "the quantity of ammonia given off from thousands of millions of putrefying animals must furnish an abundant, an everlasting source of that principle."

Important as ammonia, or its nitrogen, is conceived to be to plants, it will be seen that Liebig considers carbon not less so.

Since the appearance of the former editions of this work, the opinions of American chemists in regard to humus, have become so generally diffused, in the various Agricultural Reports, that it has not been deemed necessary to retain, in this edition, much that was appended to the second.

Professor Lindley, in speaking of humus, recognises it as "the dark substance which remains when manure is thoroughly rotted, and which colors the soil black, and without going into any technical examination of this product, we may state," he continues, "that it is a substance formed by the decay of plants, and very rich in carbon." He then quotes the expression of Liebig, that this substance, in the form in which it exists in the soil, does not yield nourishment to plants, and expresses surprise, that the author should have thought it worth his while to raise such a phantom for the mere pleasure of subduing it, for no one in Great Britain now entertains the opinion, that humus is in itself the food of plants. "Every 
student of botany is taught, that humus becomes the food of plants only by combining with the oxygen of the atmosphere and forming carbonic acid gas, and hence the great importance of preserving the roots of plants in communication with the atmosphere, which is the great source of oxygen.".

In noticing the effect of alkalies, Professor Lindley remarks, that it will lead to the explanation of many things that were inexplicable before. "When it is said, that a plant becomes tired of a soil, and we find that manuring fails to invigorate it, the destruction of alkalies in the soil, and the want of a sufficient supply of those bases in the manure, seem to offer a solution of the enigma. And in like manner the gradual decay of trees in public squares and promenades, where the soil is incessantly robbed of alkaline matter for the sake of neatness, may probably be ascribed to the same cause. So also the injurious action of weeds is explained, by their robbing the soil of that particular kind of food which is necessary to the crops among which they grow. Each will partake of the component parts of the soil, and in proportion to the vigor of their growth, that of the crop must decrease; for what one receives the others are deprived of."

"It is impossible for any one acquainted with gar, dening not to perceive the immense importance of these considerations, which show, that by adopting the modern notion, that the action of soil is chiefly mechanical, the science of horticulture has been carried backwards, instead of being advanced; and that 
the most careful examination of the chemical nature both of the soil in which a given plant grows, and of the plant itself, must be the foundation of all exact and economical methods of cultivation."

Of the importance of alkalies and salts to plants, there would seem to be no doubt, and although the credit of this discovery is in England given to Liebig, it was not new in the United States, having been announced by Dr. S. L. Dana of Lowell, and urged upon the attention of cultivators in the various Reports on the Agriculture of Massachusetts, several years ago.

As in this work many chemical and technical terms are necessarily made use of, and it may come into the hands of some persons who are not familiar with them, explanatory notes have been added which it is hoped may render the text more intelligible. The notes that are contained in the original work are distinguished by initials or abbreviations.

A valuable addition has been made in the extracts from the lectures delivered after the appearance of Liebig's work by Professor Daubeny at Oxford, on Agriculture and Rural Economy. The greater part of the third lecture is given in the Appendix, being . a summary of the practical applications of the principles developed and discussed in the body of this work.

It has been highly gratifying to the' editor, to learn from the gentleman under whose supervision the work first appeared in England, that its republication, and 
the manner in which it has been edited in this country, have met with his entire approbation. To Dr. Playfair the editor is also indebted for some valuable suggestions which were followed in preparing the second edition, and for which he would express his thanks.

A copious index, in which the original work is deficient, has been added, and numerous errors of the English press have been corrected.

The estimation in which Professor Liebig's work was viewed by the "British Association for the Advancement of Science," before whom it was brought as a Report, has been expressed by Professor Gregory, of King's College, in the remark, "that the Association had just reason to be proud of such a work, as originating in their recommendation."

On the 30 th of November, 1840 , at the anniversary meeting of the Royal Society; one of the Copley medals was awarded to the author; and on this occasion, in his absence, the President, the Marquis of Northampton, addressed his representative, Professor Daniell, as follows.

"Professor Daniell, I hold in my hand, and deliver . to you one of the Copley medals, which has been awarded by us to Professor Liebig. My principal difficulty, in the present exercise of this the most agreeable part of my official duty, is to know whether to consider M. Liebig's inquiries as most important in a chemical or in a physiological light. However that may be, he has a double claim on the scientific 
world, enhanced by the practical and useful ends to which he has turned his discoveries."

To Dr. S. L. Dana, of Lowell, the editor would acknowledge his obligations for valuable suggestions and the communication of some important additions, and also to Mr. Charles E. Buckingham, of the Medical School of this University, for his valuable assistance in correcting the proofs.

J. W. W.

Cambridgr, September, 1842. 


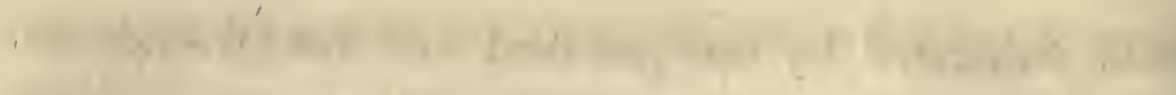

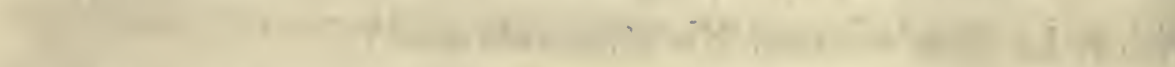

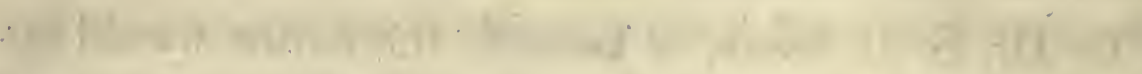

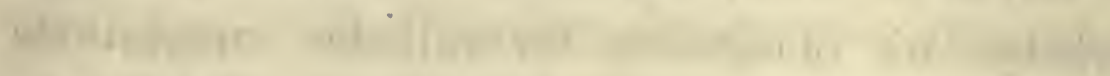

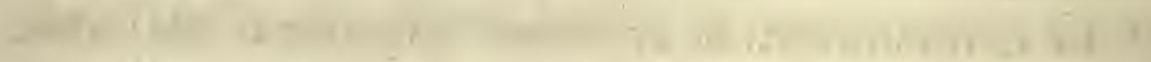

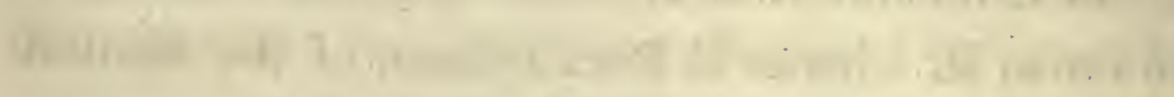

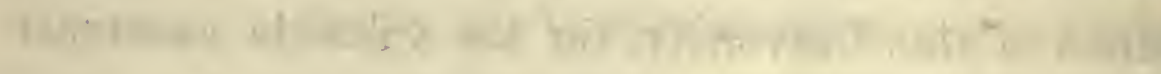
a. W - W it
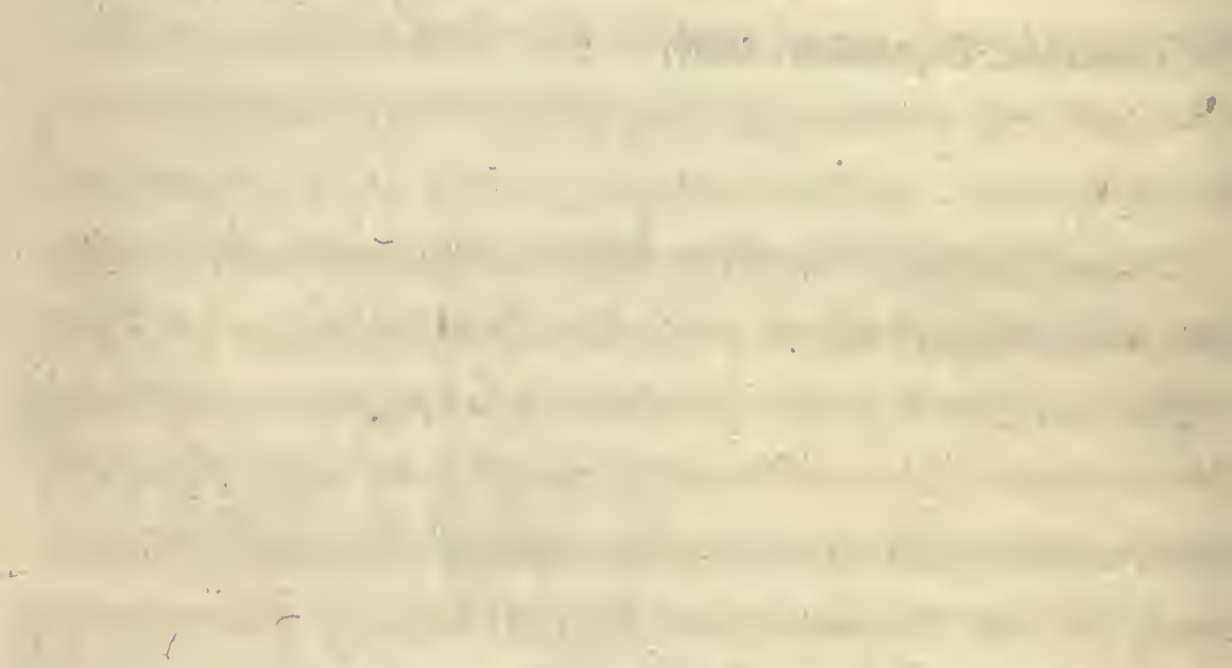

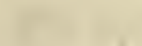
$+2$ 


\title{
THE BRITISH ASSOCIATION
}

\author{
FOR THE
}

ADVANCEMENT OF SCIENCE.

ONE of the most remarkable features of modern times is the combination of large numbers of individuals representing the whole intelligence of nations, for the express purpose of advancing science by their united efforts, of learning its progress, and of communicating new discoveries. The formation of such associations is, in itself, an evidence that they were needed.

It is not every one who is called by his situation in life to assist in extending the bounds of science; but all mankind have a claim to the blessings and benefits which accrue from its earnest cultivation. The foundation of scientific institutions is an acknowledgment of these benefits, and this acknowledgment, proceeding from whole nations, may be considered as the triumph of mind over empiricism.

Innumerable are the aids afforded to the means of life, to manufactures and to commerce, by the truths. 


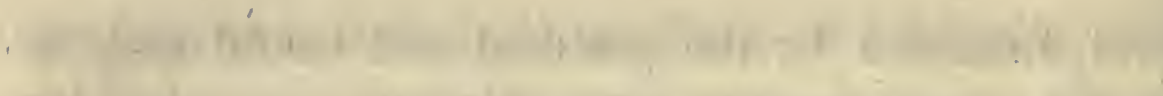

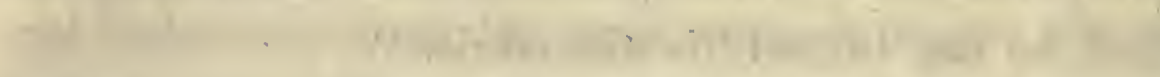

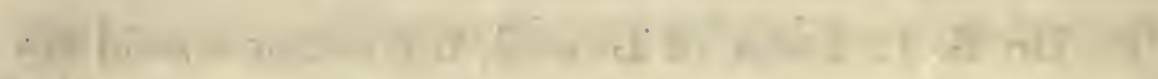

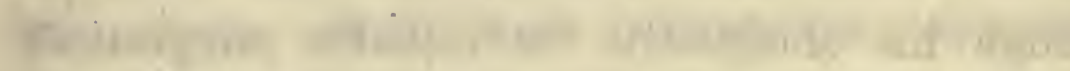

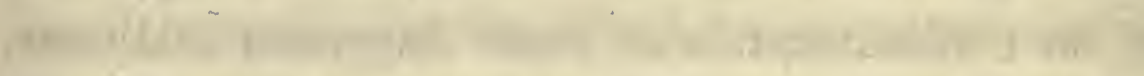

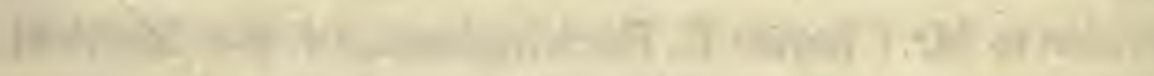
i.

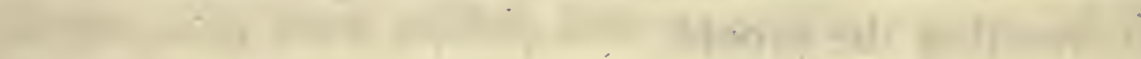
$+7+2 y^{2}$

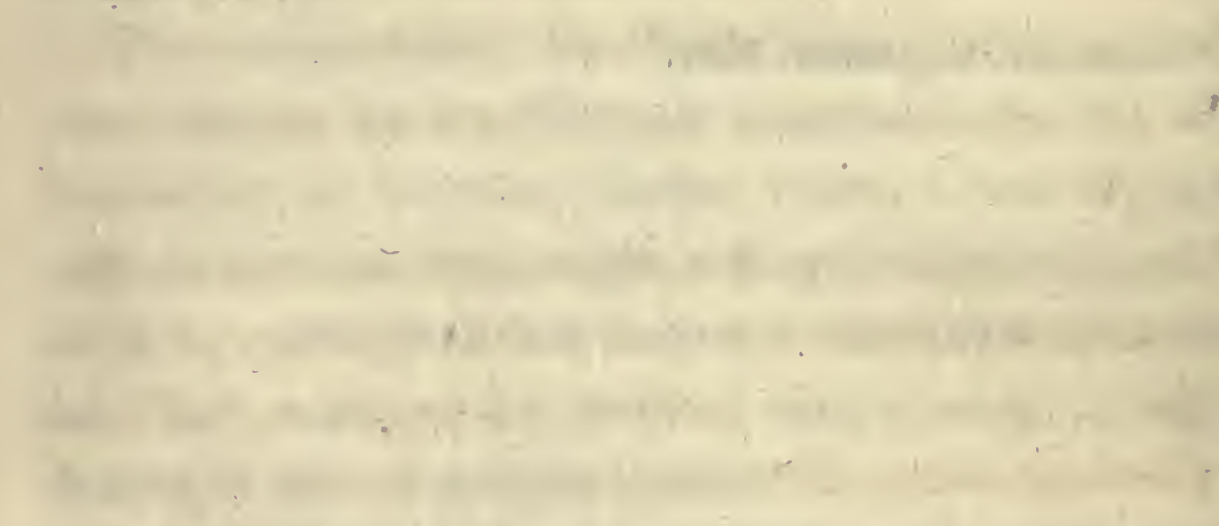

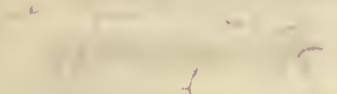

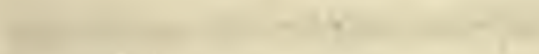

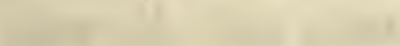

,

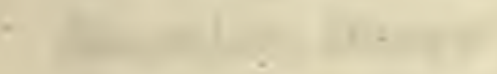

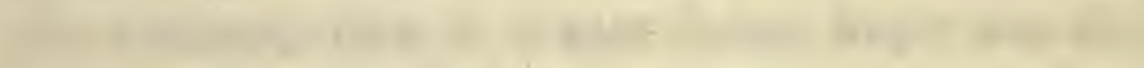

,

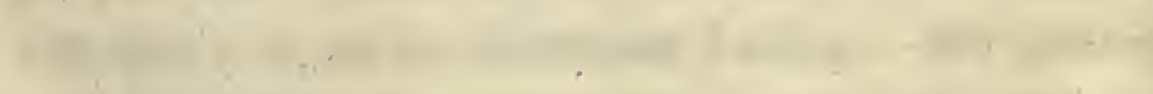
$-$

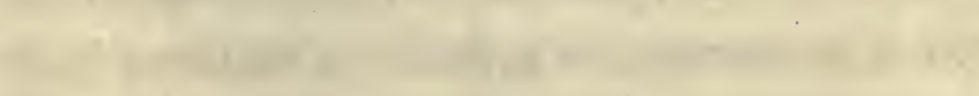

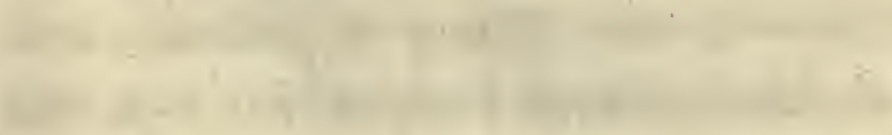




\title{
THE BRITISH ASSOCIATION
}

\author{
FOR THE
}

ADVANCEMENT OF SCIENCE.

ONE of the most remarkable features of modern times is the combination of large numbers of individuals representing the whole intelligence of nations, for the express purpose of advancing science by their united efforts, of learning its progress, and of communicating new discoveries. The formation of such associations is, in itself, an evidence that they were needed.

It is not every one who is called by his situation in life to assist in extending the bounds of science; but all mankind have a claim to the blessings and benefits which accrue from its earnest cultivation. The foundation of scientific institutions is an acknowledgment of these benefits, and this acknowledgment, proceeding from whole nations, may be considered as the triumph of mind over empiricism.

Innumerable are the aids afforded to the means of life, to manufactures and to commerce, by the truths. 
which assiduous and active inquirers have discovered and rendered capable of practical application. But it is not the mere practical utility of these truths which is of importance. Their influence upon mental culture is most beneficial; and the new views acquired by the knowledge of them enable the mind to recognise, in the phenomena of nature, proofs of an Infinite Wisdom, for the unfathomable profundity of which, language has no expression.

At one of the meetings of the chemical section of the "British Association for the Advancement of Science," the honorable task of preparing a Report upon the state of Organic Chemistry was imposed upon me. In the present work I present to the Association a part of this Report.

I have endeavored to develop, in a manner correspondent to the present state of science, the fundamental principles of Chemistry in general, and the laws of Organic Chemistry in particular, in their applications to Agriculture and Physiology; to the causes of fermentation, decay, and putrefaction; to the vinous and acetous fermentations, and to nitrification. The conversion of woody fibre into wood and mineral coal, the nature of poisons, contagions, and miasms, and the causes of their action on the living organism, have been elucidated in their chemical relations.

I shall be happy if I succeed in attracting the attention of men of science to subjects which so well merit to engage their talents and energies. Perfect Agriculture is the true foundation of all trade and in- 
dustry, - it is the foundation of the riches of states. But a rational system of Agriculture cannot be formed without the application of scientific principles; for such a system must be based on an exact acquaintance with the means of nutrition of vegetables, and with the influence of soils and action of manure upon them. This knowledge we must seek from chemistry, which teaches the mode of investigating the composition and of studying the characters of the different substances from which plants derive. their nourishment.

The chemical forces play a part in all the processes of the living animal organism ; and a number of transformations and changes in the living body are exclusively dependent on their influence. The diseases incident to the period of growth of man, contagion and contagious matters, have their analogues in many chemical processes. 'The investigation of the chemical connexion subsisting between those actions proceeding in the living body, and the transformations presented by chemical compounds, has also been a subject of my inquiries. A perfect exhaustion of this subject, so highly important to medicine, cannot be expected without the coöperation of physiologists. Hence I have merely brought forward the purely chemical part of the inquiry, and hope to attract attention to the subject.

Since the time of the immortal author of the "Agricultural Chemistry," no chemist has occupied himself in studying the applications of chemical principles to the growth of vegetables, and to organic processes. 
I have endeavored to follow the path marked out by Sir Humphry Davy, who based his conclusions only on that which was capable of inquiry and proof. This is the path of true philosophical inquiry, which promises to lead us to truth, - the proper object of our research.

In presenting this Report to the British Association I feel myself bound to convey my sincere thanks to Dr. Lyon Playfair, of St. Andrew's, for the active assistance which has been afforded me in its preparation by that intelligent young chemist during his residence in Giessen. I cannot suppress the wish, that he may succeed in being as useful, by his profound and wellgrounded knowledge of chemistry, as his talents promise.

JUSTUS LIEBIG.

GIESSEN, September 1, 1840. 


\section{EDITOR'S PREFACE}

TO

THE SECOND ENGLISH EDITION.

THE former edition of this work was prepared in the form of a Report on the present state of Organic Chemistry. The state of a science such as this could not be exhibited by a systematic treatise on organic compounds, but by showing, that the science was so far advanced as to be useful in its practical applications.

'The work was written by a Chemist, and addressed to Chemists. The author did not flatter himself, that his opinions would be so eagerly embraced by agriculturists, as circumstances have shown to be the case. Hence his language and style were less ádapted for them than for those who are conversant with the abstract details of chemical science. But the eager reception of the work by agriculturists has shown, that they possess an ardent desire to profit by the aids offered to them by Chemistry. It, therefore, became necessary to adapt the work for those who have not had an opportunity of making that science a peculiar object of study. 
The Editor has endeavored to effect this change. In doing so, it was necessary to retain the original character of the work; hence those alterations only have been made which are calculated to render the work more generally useful. It must be remembered, that the object of the author was not to write a "System of Agricultural Chemistry," but to furnish a "Treatise on the Chemistry of Agriculture." It is to be hoped, that those who are acquainted with the general doctrines of Chemistry will find no difficulty in comprehending any of the principles here developed.

The author has enriched the present edition with many valuable additions; allusion may be particularly made to the practical illustration of his principles furnished in the supplementary Chapter on Soils. The analyses of soils contained in that chapter will serve to point out the culpable negligence exhibited in the examination of English soils. Even in the analyses of professional chemists, published in detail, and with every affectation of accuracy, the estimation of the most important ingredients is neglected. How rarely do we find phosphoric acid amongst the products of their analyses? potash and soda would appear to be absent from all soils in the British territories! Yet these are invariable constituents of fertile soils, and are conditions indispensable to their fertility.

It is necessary to state, that all additions and alterations, with a few unimportant exceptions, have been 
submitted to the revision of the author. The Index at the end of the volume has been principally compiled from one furnished by Professor Webster, of Harvard University, in his American edition of this work. The editor gladly avails himself of this opportunity to thank this gentleman for the care and attention which he has displayed in superintending its republication.

Primrose, Nevember 22, 1841. 



\section{ORGANIC CHEMISTRY}

IN ITS APPLICATION TO

VEGETABLE PHYSIOLLOGY AND AGRICULTURE.

THE object of Chemistry is to examine into the composition of the numerous modifications of matter, which occur in the organic and inorganic kingdoms of nature, and to investigate the laws by which the combination and decomposition of their parts is effected.

Although material substances assume a vast variety of forms, yet chemists have not been able to detect more than fifty-five bodies which are simple, or contain only one kind of matter, and from these all other substances are produced. They are considered simple only because it has not been proved that they consist of two or more parts. The greater number of the elements occur in the inorganic kingdom. Four only are found in organic matter.

But it is evident that this limit to their number must render it more difficult to ascertain the precise circumstances, under which their union is effected, and the laws which regulate their combinations. Hence chemists have only lately turned their attention to the study of the nature of bodies generated by organized beings. A few years have, however, sufficed to throw much light upon this interesting department of science, and numerous facts have been discorered which cannot fail to be of importance in their practical applications. 
The peculiar object of organic chemistry* is to discover the chemical conditions essential to the life and perfect development of animals and vegetables, and generally to investigate all those processes of organic nature which are due to the operation of chemical laws. Now, the continued existence of all living beings is dependent on the reception by them of certain substances, which are applied to the nutrition of their frame. An inquiry, therefore, into the conditions on which the life and growth of living beings depend, involves the study of those substances which serve them as nutriment, as well as the investigation of the sources whence these substances are derived, and the changes which they undergo in the process of assimilation.

A beautiful connexion subsists between the organic and inorganic kingdoms of nature. Inorganic matter affords food to plants, and they, on the other hand, yield the means of subsistence to animals. The conditions necessary for animal and vegetable nutrition are essentially different. An animal requires for its development, and for the sustenance of its vital functions, a certain class of substances which can only be generated by organic beings possessed of life. Although many animals are entirely carnivorous, yet their primary nutriment must be derived from plants; for the animals upon which they subsist receive their nourishment from vegetable matter. But plants find new nutritive material only in inorganic substances. Hence one great end of vegetable life is to generate matter adapted for the nutrition of animals out of inorganic substances, which are not fitted for this purpose. Now the pur-

\footnotetext{
* Every vegetable and animal constitutes a machine of greater or less complexity, composed of a variety of parts dependent on each other, and acting all of them to produce a certain end. Vegetables and animals, on this account, are called organized beings; and the chemical history of those compounds which are of animal or vegetable origin, or of organic substances, is called organic chemistry. See Thomson's Chemistry of Organic Bodies, and Webster's Manual of Chemistry, 3d edit., p. 362.
} 
port of this work is, to elucidate the chemical processes engaged in the nutrition of vegetables.

The first part of it will be devoted to the examination of the matters which supply the nutriment of plants, and of the changes which these matters undergo in the living organism. The chemical compounds which afford to plants their principal constituents, viz. carbon and nitrogen, will here come under consideration, as well as the relations in which the vital functions of vegetables stand to those of the animal economy and to other phenomena of nature.

The second part of the work will treat of the chemical processes which effect the complete destruction of plants and animals after death, such as the peculiar modes of decomposition, usually described as fermentation, putrefaction, and decay; and in this part the changes which organic substances undergo in their conversion into inorganic compounds, as well as the causes which determine these changes, will become matter of inquiry. 


\section{PART I.}

OF THE CHEMICAL PROCESSES IN THE NUTRITION OF VEGETABLES.

\section{CHAPTER I.}

\section{OF THE CONSTITUENT ELEMENTS OF PLANTS.}

The ultimate constituents of plants are those which form organic matter in general, namely, Carbon, $\mathrm{Hy}-$ drogen, Nitrogen, and Oxygen. These elements are always present in plants, and produce by their union the various proximate principles of which they consist. It is, therefore, necessary, to be acquainted with their individual characters, for it is only by a correct appreciation of these that we are enabled to explain the functions which they perform in the vegetable organization.

Carbon is an elementary substance, endowed with a considerable range of affinity. With oxygen it unites in two proportions, forming the gaseous compounds known under the names of carbonic acid and carbonic oxide. The former of these is emitted in immense quantities from many volcanoes and mineral springs, and is a product of the combustion and decay of organic matter. It is subject to be decomposed by various agencies, and its elements then arrange themselves into new combinations. Carbon is familiarly known as charcoal, but in this state it is mixed with several earthy bodies; in a state of absolute purity it constitutes the diamond.*

* Wood charcoal contains about 1-50th of its weight of alkaline and earthy salts, which constitute the ashes when it is burned. 
Hydrogen (Inflammable Air) is a very important constituent of vegetable matter. It possesses a special affinity for oxygen, with which it unites and forms water. The whole of the phenomena of decay depend upon the exercise of this affinity, and many of the processes engaged in the nutrition of plants originate in the attempt to gratify it. Hydrogen, when in the state of a gas, is very combustible, and the lightest body known; but it is never found in nature in an isolated condition. Water is the most common combination in which it is presented; and it may be removed by various processes from the oxygen, with which it is united in this body.

Nitrogen ${ }^{*}$ is quite opposed in its chemical characters to the two bodies now described. Its principal characteristic is an indifference to all other substances, and an apparent reluctance to enter into combination with them. When forced by peculiar circumstances to do so, it seems to remain in the combination by a vis inertia; and very slight forces effect the disunion of these feeble compounds.

Yet nitrogen is an invariable constituent of plants, and during their life is subject to the control of the vital powers. But when the mysterious principle of

* This gas was discovered in 1772, and is called also azote or azotic gas, from the Greek, expressive of its being incapable of supporting life. The name Nitrogen was given to it from its entering into the composition of nitric acid (aqua fortis). It has been suspected to be a compound, but this has not been verified. The atmosphere is composed of four fifths nitrogen and one fifh oxygen, not, however, chemically united; it also contains a ten thousandth part of carbonic acid and watery vapor. A mixture of oxygen and nitrogen in the proportions named, exhibits the general properties of the atmosphere. Nitrogen may be obtained from common air by removing its oxygen, and from the lean part of flesh meat by boiling it in diluted nitric acid. It unites with different proportions of oxygen, and forms as many distinct compounds, viz.

\begin{tabular}{|c|c|c|c|c|}
\hline $\begin{array}{c}\text { Nitrog. } \\
\qquad 100\end{array}$ & $t$ & $\begin{array}{c}\text { Oxyg. } \\
50\end{array}$ & form & $\left\{\begin{array}{c}\text { Protoxide of Nitrogen, nitrous } \\
\text { oxide, or exhilarating gas. }\end{array}\right.$ \\
\hline 100 & + & 100 & "6 & $\left\{\begin{array}{l}\text { Binoxide of Nitrogen } \\
\text { or Nitric oxide. }\end{array}\right.$ \\
\hline $\begin{array}{l}100 \\
100 \\
100\end{array}$ & + & $\begin{array}{l}150 \\
200 \\
250\end{array}$ & "6 & $\begin{array}{l}\text { Hyponitrous acid. } \\
\text { Nitrous acid. } \\
\text { Nitric acid. }\end{array}$ \\
\hline
\end{tabular}

For other details, see Webster's Chemistry, 3d edit., p. 134, \&c. 
life has ceased to exercise its influence, this element resumes its chemical character, and materially assists in promoting the decay of vegetable matter, by escaping from the compounds of which it formed a constituent.

Oxygen, the only remaining constituent of organic matter, is a gaseous element, which plays a most important part in the economy of nature. It is the agent employed in effecting the union and disunion of a vast number of compounds. It is superior to all other elements in the extensive range of its affinities. The phenomena of combustion and decay are examples of the exercise of its power.

Oxygen is the most generally diffused element on the surface of the earth; for, besides constituting the principal part of the atmosphere which surrounds it, it is a component of almost all the earths and minerals found on its surface. In an isolated state it is a gaseous body, possessed of neither taste nor smell. It is slightly soluble in water, and hence is usually found dissolved in rain and snow, as well as in the water of running streams.

Such are the principal characters of the elements which constitute organic matter; but it remains for us to consider in what form they are united in plants.

The substances which constitute the principal mass of every vegetable are compounds of carbon with oxygen and hydrogen, in the proper relative proportions for forming water. Woody fibre, starch, sugar, and gum, for example, are such compounds of carbon with the elements of water. In another class of substances containing carbon as an element, oxygen and hydrogen are again present; but the proportion of oxygen is greater than would be required for producing water by union with the hydrogen. The numerous organic acids met with in plants belong, with few exceptions, to this class.

A third class of vegetable compounds contains carbon and hydrogen, but no oxygen, or less of that element than would be required to convert all the 
hydrogen into water. These may be regarded as compounds of carbon with the elements of water, and an excess of hydrogen. Such are the volatile and fixed oils, wax, and the resins. Many of them have acid characters.

The juices of all vegetables contain organic acids, generally combined with the inorganic bases, or metallic oxides; for these metallic oxides exist in every plant, and may be detected in its ashes after incineration.

Nitrogen is an element of vegetable albumen and gluten; it is a constituent of the acid, and of what are termed the "indifferent substances" of plants, as well as of those peculiar vegetable compounds which possess all the properties of metallic oxides, and are known as "organic bases."

Estimated by its proportional weight, nitrogen forms only a very small part of plants; but it is never entirely absent from any part of them. Even when it does not absolutely enter into the composition of a particular part or organ, it is always to be found in the fluids which pervade it.

It follows from the facts thus far detailed, that the development of a plant requires the presence, first, of substances containing carbon and nitrogen, and capable of yielding these elements to the growing organism; secondly, of water and its elements; and lastly, of a soil to furnish the inorganic matters which are likewise essential to vegetable life.

OF THE COMPOSITION OF THE ATMOSPHERE.

In the normal state of growth, plants can only derive their nourishment from the atmosphere and the soil. Hence it is of importance to be acquainted with the composition of these, in order that we may be enabled to judge from which of their constituents the nourishment is afforded.

The composition of the atmosphere has been exam- 
ined by many chemists with great care, and the results of their researches have shown, that its principal constituents are always present in the same proportion. These are the two gases, oxygen and nitrogen, the general properties of which have been already described. One hundred parts, by weight, of atmospheric air contain $23 \cdot 1$ parts of oxygen, and 76:9 parts of nitrogen; or 100 volumes of air contain nearly 21 volumes of oxygen gas. From the extensive range of affinity which this gas possesses, it is obvious, that were it alone to constitute our atmosphere, and left unchecked to exert its powerful effects, all nature would be one scene of universal destruction. It is on this account that nitrogen is present in the air in so large proportion. It is peculiarly adapted for this purpose, as it does not possess any disposition to unite with oxygen, and exerts no action upon the processes proceeding on the earth. These two gases are intimately mixed, by virtue of a property which all gases possess in common, of diffusing themselves equally through every part of another gas, with which they are placed in contact.

Although oxygen and nitrogen form the principal constituents of the atmosphere, yet they are not the only substances found in it. Watery vapor and carbonic acid gas materially modify its properties. 'The former of these falls upon the earth as rain, and brings with it any soluble matter which it meets in its passage through the air.

Carbonic acid gas is discharged in immense quantities from the active volcanoes of America, and from many of the mineral springs which abound in various parts of Europe; it is also generated during the combustion and decay of organic matter. It is not, therefore, surprising that it should have, been detected in every part of the atmosphere in which its presence has been looked for. Saussure found it even in the air on the summit of Mont Blanc, which is covered with perpetual snow, and where it could 
not have been produced by the immediate agency of vegetable matter. Carbonic acid gas performs a most important part in the process of vegetable nutrition, the consideration of which belongs to another part of the work.

Carbonic acid, water, and ammonia (a compound of hydrogen and nitrogen) are the final products of the decay of animal and vegetable matter. In an isolated condition, they usually exist in the gaseous form. Hence, on their formation, they must escape into the atmosphere. But ammonia has not hitherto been enumerated amongst the constituents of the air, although, according to our view, it can never be absent. The reason of this is, that it exists in extremely minute quantity in the amount of air usually subjected to experiment in chemical analysis; it has consequently escaped detection. But rain which falls through a large extent of air, carries down in solution all that remains in suspension in it. Now ammonia always exists in rain-water, and from this fact we must conclude that it is invariably present in the atmosphere. Nor can we be surprised at its presence when we consider that many volcanoes now in activity emit large quantities of it.* This subject will, however, be discussed more fully in another part of the work.

Such are the principal constituents of the atmosphere from which plants derive their nourishment; for although other matters are supposed to exist in it in minute quantity, yet they do not exercise any influence on vegetation, nor has even their presence been satisfactorily demonstrated.

\section{OF SOILS.}

A soil may be considered a magazine of inorganic matters, which are prepared by the plant to suit the

* The annual evolution of carbonic acid from springs and fissures in the ancient volcanic district of the Eifel, on the Rhine, has been estimated by Bischof, at not less than 100,000 tons, containing 27,000 tons of carbon. 
purposes for which they are destined in its nutrition. The composition and uses of such substances cannot, however, be studied with advantage, until we have considered the manner in which the organic matter is obtained by plants.

Some virgin soils, such as those of America, contain vegetable matter in large proportion; and as these have been found eminently adapted for the cultivation of most plants, the organic matter contained in them has naturally been recognised as the cause of their fertility. To this matter, the term "vegetable mould" or humus has been applied. Indeed, this peculiar substance appears to play such an important part in the phenomena of vegetation, that vegetable physiologists have been induced to ascribe the fertility of every soil to its presence. It is believed by many to be the principal nutriment of plants, and is supposed to be extracted by them from the soil in which they grow. It is itself the product of the decay of regetable matter, and must therefore contain many of the constituents which are found in plants during life. Its action will therefore be examined in considering whence these constituents are derived.

\section{CHAPTER II. \\ OF , THE ASSIMILATION OF CARBON.}

COMPOSITION OF HUMUS.

The humus, to which allusion has been made, is described by chemists as a brown substance easily soluble in alkalies, but only slightly so in water, and produced during the decomposition of vegetable matters by the action of acids or alkalies. It has, however, received various names according to the different external characters and chemical properties which it presents. Thus, ulmin, humic acid, coal of 
humus, and humin, are names applied to modifications of humus. They are obtained by treating peat, woody fibre, soot, or brown coal with alkalies; by decomposing sugar, starch, or sugar of milk by means of acids; or by exposing alkaline solutions of tannic and gallic acids to the action of the air.

The modifications of humus which are soluble in alkalies, are called humic acid; while those which are insoluble have received the designations of humin and coal of humus.*

The names given to these substances might cause it to be supposed that their composition is identical. But a more erroneous notion could not be entertained; since even sugar, acetic acid, and resin do not differ more widely in the proportions of their constituent elements, than do the various modifications of humus.

\section{Humic acid formed by the action of hydrate $f$ of}

* The soluble matters were formerly called by the eminent Swedish chemist Berzelius, extract of humus, and the insoluble geine (from the Greek $\gamma \tilde{\eta}$, the eurth), also apotheme and carbonaceous humus. This substance is now known to be composed of various ingredients, and of these the two acids, which have received the names of Crenic and Apocrenic, are particularly interesting.

See Professor Hitchcock's Report, and American Journal of Science, Vol. XXXVI., Art. XII.

Dr. S. L. Dana considers geine as forming the basis of all the nourishing part of all vegetable manures, and, in the three states of "vegetable extract, geine, and carbonaceous mould," to be the principle which gives fertility to soils long after the action of common manures has ceased. See Report on the reexamination of the Economical Geology of Massachusetts. In the Third Report on the Agriculture of the State of Massachusetts, 1840, Dr. Dana remarks, that geine "is the decomposed organic matter of the soil. It is the product of putrefaction; continually subjected to air and moisture, it is finally wholly dissipated in air, leaving only the inorganic bases of the plant, with which it was once combined. Now, whether we consider this as a simple substance, or composed of several others, called crenic, apocrenic, puteanic, ulmic acids, glairin, apotheme, extract, humus, or mould, agriculture ever has and probably ever will consider it one and the same thing, requiring always similar treatment to produce it; similar treatment to render it soluble when produced; similar treatment to render it an effectual manure. It is the end of all compost heaps to produce soluble geine, no matter how compound our chemistry may teach this substance to be." Page 191.

+ Hydrates are compounds of oxides, salts, \&c., with definite quan. tities of water, - a substance from which all the water has been removed is anhydrous. Even after exposure to a red heat, caustic potash retains water. 
potash upon sawdust contains, according to the accurate analysis of Peligot, 72 per cent. of carbon, while the humic acid obtained from turf and brown coal contains, according to Sprengel, only 58 per cent.; that produced by the action of dilute sulphuric acid upon sugar, 57 per cent. according to Malaguti; and that, lastly, which is obtained from sugar or from starch, by means of muriatic acid, according to the analysis of Stein, 64 per cent. All these analyses have been repeated with care and accuracy, and the proportion of carbon in the respective cases has been found to agree with the estimates of the different chemists above mentioned; so that there is no reason to ascribe the difference in this respect between the varieties of humus to the mere difference in the methods of analysis or degrees of expertness of the operators. Malaguti states, moreover, that humic acid contains an equal number of equivalents of oxygen and hydrogen, that is to say, that these elements exist in it in the proportions for forming water; while, according to Sprengel, the oxygen is in excess, and Peligot even estimates the quantity of oxygen at 14 equivalents, and the hydrogen at only 6 equivalents, making the deficiency of hydrogen as great as 8 equivalents. And although Mulder* has very recently explained many of these conflicting results, by showing that there are several kinds of humus and humic acids essentially distinct in their characters, and fixed in their composition, yet he has afforded no proof that the definite compounds obtained by him really exist, as such, in the soil. On the contrary, they appear to have been formed by the action of the potash and ammonia, which he employed in their preparation.

It is quite evident, therefore, that chemists have been in the habit of designating all products of the decomposition of organic bodies which had a brown or brownish-black. color by the names of humic

* Bulletin des Scienc. Phys. et Natur. de Neerl. 1840, p. 1-102. 
acid or humin, according as they were soluble or insoluble in alkalies; although in their composition and mode of origin, the substances thus confounded might be in no way allied.

Not the slightest ground exists for the belief that one or other of these artificial products of the decomposition of vegetable matters exists in nature in the form and endowed with the properties of the vegetable constituents of mould; there is not the shadow of a proof that one of them exerts any influence on the growth of plants either in the way of nourishment or otherwise.

Vegetable physiologists have, without any apparent reason, imputed the known properties of the humus and humic acids of chemists to that constituent of mould which has received the same name, and in this way have been led to their theoretical notions respecting the functions of the latter substance in vegetation.

The opinion, that the substance called humus is extracted from the soil by the roots of plants, and that the carbon entering into its composition serves in some form or other to nourish their tissues, is considered by many as so firmly established, that any new argument in its favor has been deemed unnecessary; the obvious difference in the growth of plants, according to the known abundance or scarcity of humus in the soil, seemed to afford incontestable proof of its correctness.*

Yet, this position, when submitted to a strict examination, is found to be untenable, and it becomes evident, from most conclusive proofs, that humus, in the form in which it exists in the soil, does not yield the smallest nourishment to plants.

The adherence to the above incorrect opinion has

* This remark applies more to German than to English botanists and physiologists. In England, the idea that humus, as such, affords nourishment to plants is by no means general; but on the Continent, the views of Berzelius on this subject have been almost universally adopted. - ED. 
hitherto rendered it impossible for the true theory of the nutritive process in vegetables to become known, and has thus deprived us of our best guide to a rational practice in agriculture. Any great improvement in that most important of all arts is inconceivable, without a deeper and more perfect acquaintance with the substances which nourish plants, and with the sources whence they are derived; and no other cause can be discovered to account for the fluctuating and uncertain state of our knowledge on this subject up to the present time, than that modern physiology has not kept pace with the rapid progress of chemistry.

In the following inquiry, we shall suppose the $h u$ mus of vegetable physiologists to be really endowed with the properties recognised by chemists in the brownish black deposits, which they obtain by precipitating an alkaline decoction of mould or peat by means of acids, and which they name humic acid.*

Humic acid, when first precipitated, is a flocculent substance, is soluble in 2500 times its weight of water, and combines with alkalies, lime and magnesia, forming compounds of the same degree of solubility. (Sprengel.)

Vegetable physiologists agree in the supposition that by the aid of water humus is rendered capable

* The extract obtained by Berzelius from black, brownish soils has been designated as humic extract, in some cases with a substance called glairin. The glairin is described by Thomson as a peculiar substance which has been observed in certain sulphureous mineral waters, and was first noticed by Vauquelin (Ann. de Chim. XXXIX. 17:3), who described several of its properties and considered it analogous to gelatin. An account of it was drawn up by M. Anglada, of Montpellier, and communicated to the Royal Academy of Medicine of Paris, in 1827. It gelatinizes with water when sufficiently concentrated. Sometimes it is white, and at others of a red color: when dried it shrinks to $\frac{1}{60}$ th of its bulk when moist. It saturates ammonia, and decomposes several me. tallic salts. It is destitute of smell and taste. It does not glue substances together like gelatin and albumen. It yields ammonia by decomposition, and is capable of putrefaction like animal bodies. The general opinion is, that it is of vegetable origin, and allied to the genus tremella, though its existence in mineral waters has not been accounted for. Thomson's Organic Chemistry, 694. I found it very abundant about the hot sulphureous waters of the island of St. Michael, Azores. - $W$. 
of being absorbed by the roots of plants. But according to the observation of chemists, humic acid is soluble only when newly precipitated, and becomes completely insoluble when dried in the air, or when exposed in the moist state to the freezing temperature. (Sprengel.)

Both the cold of winter and the heat of summer therefore are destructive of the solubility of humic acid, and at the same time of its capability of being assimilated by plants. So that, if it is absorbed by plants, it must be in some altered form.

The correctness of these observations is easily demonstrated by treating a portion of good mould with cold water. The fluid remains colorless, and is found to have dissolved less than 100,000 part of its weight of organic matters, and to contain merely the salts which are present in rain-water.

Decayed oak-wood, likewise, of which humic acid is the principal constituent, was found by Berzelius to yieid to cold water only slight traces of soluble materials; and I have myself verified this observation on the decayed wood of beech and fir.

These facts, which show that humic acid, in its unaltered condition, cannot serve for the nourishment of plants, have not escaped the notice of physiologists ; and hence they have assumed that the lime or the different alkalies, found in the ashes of vegetables, render soluble the humic acid and fit it for the process of assimilation.

Alkalies and alkaline earths do exist in the different kinds of soil in sufficient quantity to form such soluble compounds with the humic acid.

Now, let us suppose that humic acid is absorbed by plants in the form of that salt which contains the largest proportion of humic acid, namely, in the form of humate of lime, and then, from the known quantity of the alkaline bases contained in the ashes of plants, let us calculate the amount of humic acid which might be assimilated in this manner. Let us admit, likewise, that potash, soda, and the oxides of iron 
and manganese have the same capacity of saturation as lime with respect to humic acid, and then we may take as the basis of our calculation the analysis of M. Berthier, who found that 1000 lbs. of dry fir-wood yielded $4 \mathrm{lbs}$. of ashes, and that in every $100 \mathrm{lbs}$. of these ashes, after the chloride of potassium and sulphate of potash were extracted, $53 \mathrm{lbs}$. consisted of the basic metallic oxides, potash, soda, lime, magnesia, iron, and manganese.

One Hessian acre* of woodland yields annually, according to Dr. Heyer, on an average, $2920 \mathrm{lbs}$. of dry fir-wood, which contain $6.17 \mathrm{lbs}$. of metallic oxides.

Now, according to the estimates of Malaguti and Sprengel, $1 \mathrm{lb}$ of lime combines chemically with 12 lbs. of humic acid; $6.17 \mathrm{lbs}$. of the metallic oxides would accordingly introduce into the trees 74.04 of humic acid, which, admitting humic acid to contain 58 per cent. of carbon, would correspond to $100 \mathrm{lbs}$. of dry wood. But we have seen that $2920 \mathrm{lbs}$. of fir-wood are really produced.

Again, if the quantity of humic acid which might be introduced into wheat in the form of humates is calculated from the known proportion of metallic oxides existing in wheat straw, (the sulphates and chlorides also contained in the ashes of the straw not being included,) it will be found that the wheat growing on 1 Hessian acre would receive in that way $63 \mathrm{lbs}$. of humic acid, corresponding to $93.6 \mathrm{lbs}$. of woody fibre. But the extent of land just mentioned produces, independently of the roots and grain, $1961 \mathrm{lbs}$. of straw, the composition of which is the same as that of woody fibre.

It has been taken for granted in these calculations that the basic metallic oxides which have served to introduce humic acid into the plants do not return to the soil, since it is certain that they remain fixed

* One Hessian acre is equal to 40,000 square feet, Hessian, or 26,910 square feet, English measure. $-P$. 
in the parts newly formed during the process of growth.

Let us now calculate the quantity of humic acid which plants can receive under the most favorable circumstances, viz., through the agency of rainwater.

The quantity of rain which falls at Erfurt, one of the most fertile districts of Germany, during the months of April, May, June, and July, is stated by Schubler to be $19.3 \mathrm{lbs}$. over every square foot of surface; 1 Hessian acre, or 26,910 square feet, consequently receive $519,363 \mathrm{lbs}$. of rain-water.

If, now, we suppose that the whole quantity of this rain is taken up by the roots of a summer plant, which ripens four months after it is planted, so that not a pound of this water evaporates except from the leaves of the plant; and if we further assume that the water thus absorbed is saturated with humate of lime (the most soluble of the humates, and that which contains the largest proportion of humic acid); then the plants thus nourished would not receive more than $330 \mathrm{lbs}$. of humic acid, since: one part of humate of lime requires 2500 parts of water for solution.

But the extent of land which we have mentioned produces $2843 \mathrm{lbs}$. of corn (in grain and straw, the roots not included), or $22,000 \mathrm{lbs}$. of beet-ront (without the leaves and small radical fibres). It is quite evident that the $330 \mathrm{lbs}$. of humic acid, supposed to be absorbed, cannot account for the quantity of carbon contained in the roots and leaves alone, even if the supposition were correct, that the whole of the rain-water was absorbed by the plants. But since it is known that only a small portion of the rain-water which falls upon the surface of the earth evaporates through plants, the quantity of carbon which can be conveyed into them in any conceivable manner by means of humic acid must be extremely trifling, in comparison with that actually. produced in vegetation. 
Other considerations of a higher nature confute the common view respecting the nutritive office of humic acid, in a manner so clear and conclusive that it is difficult to conceive how it could have been so generally adopted.

Fertile land produces carbon in the form of wood, hay, grain, and other kinds of growth, the masses of which differ in a remarkable degree.

$2920 \mathrm{lbs}$. of firs, pines, beeches, \&c. grow as wood upon one Hessian acre of forest-land with an average soil. The same superficies yields $2755 \mathrm{lbs}$. of hay.

A similar surface of corn-land gives from 19,000 to $22,004 \mathrm{lbs}$. of beet-root, or $881 \mathrm{lbs}$. of rye, and $1961 \mathrm{lbs}$. of straw, 160 sheaves of $15.4 \mathrm{lbs}$. each, in all, $2843 \mathrm{lbs}$.

One hundred parts of dry fir-wood contain 38 parts of carbon; therefore, 2920 lbs. contain 1109 lbs. of carbon.

One hundred parts of hay, ${ }^{*}$ dried in air, contain 44.31 parts carbon. Accordingly, $2755 \mathrm{lbs}$. of hay contain $1110 \mathrm{lbs}$. of carbon.

Beet-roots contain from 89 to 89.5 parts water, and from 10.5 to 11 parts solid matter, which consists of from 8 to 9 per cent. sugar, and from 2 to $2 \frac{1}{2}$ per cent. cellular tissue. Sugar contains 42.4 per cent.; cellular tissue, 47 per cent. of carbon.

$22,004 \mathrm{lbs}$. of beet-root, therefore, if they contain 9 per cent. of sugar, and 2 per cent. of cellular tissue, would yield 1031 lbs. of carbon, of which 833 lbs. would be due to the sugar, and $198 \mathrm{lbs}$. to the cellular tissue; the carbon of the leaves and small roots not being included in the calculation.

One hundred parts of straw, $\dagger$ dried in air, contain

* 100 parts of hay, dried at $100^{\circ} \mathrm{C}$. $\left(212^{\circ} \mathrm{F}\right.$.) and burned with oxide of copper in a stream of oxygen gas, yielded 51.93 water, 165.8 carbonic acid, and 6.82 of ashes. This gives 45.87 carbon, 5.76 hydrogen, 31.55 oxygen, and 6.82 ashes. Hay, dried in the air, loses $11.2 \mathrm{p}$. c. water at $100^{\circ} \mathrm{C}$. (212 F. ) - (Dr. Will.)

+ Straw analyzed in the same manner, and dried at $100^{\circ} \mathrm{C}$, gave 46.37 p. c. of carbon, 5.68 p. c. of hydrogen, 43.93 p. c. of oxygen, and 4.02 p. c. of ashes. Straw dried in the air at $100^{\circ} \mathrm{C}$. lost 18 p. c. of water. - (Dr. Will.) 
38 per cent. of carbon; therefore 1961 lbs. of straw contain 745 lbs. of carbon. One hundred parts of corn contain 43 parts of carbon; 882 lbs. must therefore contain 379 lbs., - in all, 1124 lbs. of carbon.

26,910 square feet of wood and meadow land produce, consequently, 1109 lbs. of carbon; while the same extent of arable land yields in beet-root, without leaves, 1032 lbs., or in corn, 1124 lbs.

It must be concluded from these incontestable facts, that equal surfaces of cultivated land of an average fertility produce equal quantities of carbon; yet, how unlike have been the different conditions of the growth of the plants from which this has been deduced!

Let us now inquire whence the grass in a meadow, or the wood in a forest, receives its carbon, since there no manure - no carbon - has been given to it as nourishment? and how it happens, that the soil, thus exhausted, instead of becoming poorer, becomes every year richer in this element?

A certain quantity of carbon is taken every year from the forest or meadow, in the form of wood or hay, and, in spite of this, the quantity of carbon in the soil augments; it becomes richer in humus.

It is said that in fields and orchards all the carbon which may have been taken away as herbs, as straw, as seeds, or as fruit, is replaced by means of manure; and yet this soil produces no more carbon than that of the forest or meadow, where it is never replaced. It cannot be conceived that the laws for the nutrition of plants are changed by culture, - that the sources of carbon for fruit or grain, and for grass or trees, are different.

It is not denied that manure exercises an influence upon the development of plants; but it may be affirmed with positive certainty, that it neither serves for the production of the carbon, nor has any influence upon it, because we find that the quantity of carbon produced by manured lands is not greater 
than that yielded by lands which are not manured. The discussion as to the manner in which manure acts has nothing to do with the present question, which is, the origin of the carbon. The carbon must be derived from other sources; and as the soil does not yield it, it can only be extracted from the atmosphere.

In attempting to explain the origin of carbon in plants, it has never been considered that the question is intimately connected with that of the origin of humus. It is universally admitted that hurnus arises from the decay of plants. No primitive humus, therefore, can have existed, - for plants must have preceded the humus.

Now, whence did the first vegetables derive their carbon? and in what form is the carbon contained in the atmosphere?

These two questions involve the consideration of two most remarkable natural phenomena, which by their reciprocal and uninterrupted influence maintain the life of the individual animals and vegetables, and the continued existence of both kingdoms of organic nature.

One of these questions is connected with the invariable condition of the air with respect to oxygen. One hundred volumes of air have been found, at every period and in every climate, to contain 21 volumes of oxygen, with such small deviations that they must be ascribed to errors of observation.

Although the absolute quantity of oxygen contained in the atmosphere appears very great when represented by numbers, yet it is not inexhaustible. One man consumes by respiration 25 cubic feet of oxygen in 24 hours; $10 \mathrm{cwt}$. of charcoal consume 32,066 cubic feet of oxygen during its combustion; and a small town like Giessen (with about 7000 inhabitants) extracts yearly from the air, by the wood employed as fuel, more than 551 millions of cubic feet of this gas.

When we consider facts such as these, our former 
statement, that the quantity of oxygen in the atmosphere does not diminish in the course of ages, ${ }^{*}$ that the air at the present day, for example, does not contain less oxygen than that found in jars buried for 1800 years in Pompeii, - appears quite incomprehensible, unless some source exists whence the oxygen abstracted is replaced. How does it happen, then, that the proportion of oxygen in the atmosphere is thus invariable?

The answer to this question depends upon another; namely, what becomes of the carbonic acid, which is produced during the respiration of animals, and by the process of combustion? A cubic foot of oxygen gas, by uniting with carbon so as to form carbonic acid, does not change its volume. The billions of cubic feet of oxygen extracted from the atmosphere, produce the same number of billions of cubic feet

* If the atmosphere possessed, in its whole extent, the same density as it does on the surface of the sea, it would have a height of 24,555 Parisian feet; but it contains the vapor of water, so that we may assume its height to be one geographical mile $=22,843$ Parisian feet. Now the radius of the earth is equal to 860 geographical miles; hence the

Volume of the atmosphere $=9,307,500$ cubic miles.

$\begin{aligned} \text { Volume of oxygen } . \quad & =\text { cube of } 210 \cdot 4 \text { miles. } \\ & =1,954,578 \text { cubic miles. } \\ & =\text { cube of } 125 \text { miles. } \\ \text { Volume of carbonic acid } & =3,862 \cdot 7 \text { cubic miles. } \\ & =\text { cube of } 15.7 \text { miles. }\end{aligned}$

The maximum of the carbonic acid contained in the atmosphere has not here been adopted, but the mean, which is equal to 0.000415 . (L.) The weight of carbon which presses upon each square inch of the earth's surface being $17 \cdot 39$ grains, on an acre of land will be 7 tons. - (Johnston.)

A man daily consumes 45,000 cubic inches (Parisian). A man yearly consumes $9505 \cdot 2$ cubic feet. 100 million men yearly consume $9,505,200,000,000$ cubic feet.

Hence a thousand million men yearly consume 0.79745 cubic miles of oxygen. But the air is rendered incapable of supporting the process of respiration, when the quantity of its oxygen is decreased 12 per cent.; so that a thousand million men would make the air unfit for respiration in a million years. The consumption of oxygen by animals, and by the process of combustion, is not introduced into the calculation.

When the air returns from the lungs, the carbonic acid gas amounts, on an average, to $\frac{1}{25}$ th of the whole; or its quantity is increased one hundred times. - (Johnston.) A full grown man gives off from his lungs, in the course of a year, upwards of $100 \mathrm{lbs}$. of carbon. It is estimated by Johnston, that at least one third of the carbon of the food of men is daily returned to the air. 
of carbonic acid, which immediately supply its place.

The most exact and most recent experiments of De Saussure, made in every seasor for a space of three years, have shown, that the air contains on an average 0.000415 of its own volume of carbonic acid gas; so that, allowing for the inaccuracies of the experiments, which must diminish the quantity obtained, the proportion of carbonic acid in the atmosphere may be regarded as nearly equal to $\frac{1}{1000}$ th part of its weight. The quantity varies according to the seasons; but the yearly average remains continually the same.

We have no reason to believe that this proportion was less in past ages; and nevertheless, the immense masses of carbonic acid which annually flow in to the atmosphere from so many sources, ought perceptibly to increase its quantity from year to year. But we find that all earlier observers describe its volume as from one-half to ten times. greater than that which it has at the present time; so that we can hence at most conclude that it has diminished.

It is quite evident that the quantities of carbonic acid and oxygen in the atmosphere, which remain unchanged by lapse of time, must stand in some fixed relation to one another; a cause must exist which prevents the increase of carbonic acid by removing that which is constantly forming; and there must be some means of replacing the oxygen, which is removed from the air by the processes of combustion and putrefaction, as well as by the respiration of animals.

Both these causes are united in the process of vegetable life.

The facts which we have stated in the preceding pages prove, that the carbon of plants must be derived exclusively from the atmosphere. Now, carbon exists in the atmosphere only in the form of carbonic acid, and therefore in a state of combination with oxygen. 
It has been already mentioned likewise, that carbon and the elements of water form the principal constituents of vegetables; the quantity of the substances which do not possess this composition being in a very small proportion. Now, the relative quantity of oxygen in the whole mass is less than in carbonic acid; for the latter contains two equivalents of oxygen, whilst one only is required to unite with hydrogen in the proportion to form water. The vegetable products which contain oxygen in larger proportion than this, are, comparatively, few in number; indeed in many the hydrogen is in great excess. It is obvious, that when the hydrogen of water is assimilated by a plant, the oxygen in combination with it must be liberated, and will afford a quantity of this element sufficient for the wants of the plants. If this be the case, the oxygen contained in the carbonic acid is quite unnecessary in the process of vegetable nutrition, and it will consequently escape into the atmosphere in a gaseous form. It is therefore certain, that plants must possess the power of decomposing carbonic acid, since they appropriate its carbon for their own use. The formation of their principal component substances must necessarily be attended with the separation of the carbon of the carbonic acid from the oxygen, which must be returned to the atmosphere, whilst the carbon enters into combination. with water or its elements. The atmosphere must thus receive a volume of oxygen for every volume of carbonic acid which has been decomposed.

This remarkable property of plants has been demonstrated in the most certain manner, and it is in the power of every person to convince himself of its existence. The leaves and other green parts of a plant absorb carbonic acid, and emit an equal volume of oxygen. They possess this property quite independently of the plant; for if, after being separated from the stem, they are placed in water containing carbonic acid, and exposed in that condition to the 
sun's light, the carbonic acid is, after a time, found to have disappeared entirely from the water. If the experiment is conducted under a glass receiver filled with water, the oxygen emitted from the plant may be collected and examined. When no more oxygen gas is evolved, it is a sign that all the dissolved carbonic acid is decomposed; but the operation recommences if a new portion of it is added.

Plants do not emit gas when placed in water which either is free from carbonic acid, or contains an alkali that protects it from assimilation.

These observations were first made by Priestley and Sennebier. The excellent experiments of De Saussure have further shown, that plants increase in weight during the decomposition of carbonic acid and separation of oxygen. This increase in weight is greater than can be accounted for by the quantity of carbon assimilated; a fact which confirms the view, that the elements of water are assimilated at the same time.

The life of plants is closely connected with that of animals, in a most simple manner, and for a wise and sublime purpose.

The presence of a rich and luxuriant vegetation may be conceived without the concurrence of animal life, but the existence of animals is undoubtedly dependent upon the life and development of plants.

Plants not only afford the means of nutrition for the growth and continuance of animal organization, but they likewise furnish that which is essential for the support of the important vital process of respiration; for besides separating all noxious matters from the atmosphere, they are an inexhaustible source of pure oxygen, which supplies the loss which the air is constantly sustaining. Animals on the other hand expire carbon, which plants inspire; and thus the composition of the medium in which both exist, namely, the atmosphere, is maintained constantly unchanged.

It may be asked, - Is the quantity of carbonic acid 
in the atmosphere, which scarcely amounts to $\frac{1}{10}$ th per cent., sufficient for the wants of the whole vegetation on the surface of the earth, - is it possible that the carbon of plants has its origin from the air alone? This question is very easily answered. It is known, that a column of air of 2441 lbs. weight rests upon every square Hessian foot $(=0.567$ square foot English) of the surface of the earth; the diameter of the earth and its superficies are likewise known, so that the weight of the atmosphere can be calculated with the greatest exactness. 'The thousandth part of this is carbonic acid, which contains upwards of 27 per cent. carbon. By this calculation it can be shown, that the atmosphere contains 3306 billion lbs. of carbon; a quantity which amounts to more than the weight of all the plants, and of all the strata of mineral and brown coal, which exist upon the earth. This carbon is, therefore, more than adequate to all the purposes for which it is required. The quantity of carbon contained in sea-water is proportionally still greater.

If, for the sake of argument, we suppose the superficies of the leaves and other green parts of plants, by which the absorption of carbonic acid is effected, to be double that of the soil upon which they grow, a supposition which is much under the truth in the case of woods, meadows, and corn-fields ; and if we further suppose that carbonic acid equal to 0.00067 of the volume of the air, or $\frac{1}{1000}$ th of its weight, is abstracted from it during every second of time, for eight hours daily, by a field of 53,820 square feet ( $=2$ Hessian acres); then those leaves would receive 1102 lbs. of carbon in 200 days.*

* The quantity of carbonic acid which can be extracted from the air in a given time, is shown by the following calculation. During the white-washing of a small chamber, the superficies of the walls and roof of which we will suppose to be 105 square metres, and which receives six coats of lime in four days, carbonic acid is abstracted from the air, and the lime is consequently converted, on the surface, into a carbonate. It has been accurately determined that one square decimetre receives in this way, a coating of carbonate of lime which weighs 0.732 grammes. Upon the 105 square metres already mentioned there nust 
But it is inconceivable, that the functions of the organs. of a plant can cease for any one moment during its life. The roots and other parts of it, which possess the same power, absorb constantly water and carbonic acid. This power is independent of solar light. During the day, when plants are in the shade, and during the night, carbonic acid is accumulated in all parts of their structure; and the assimilation of the carbon and the exhalation of oxygen commence from the instant that the rays of the sun strike them. As soon as a young plant breaks through the surface of the ground, it begins to acquire color from the top downwards; and the true formation of woody tissue commences at the same time.*

The proper, constant, and inexhaustible sources of oxygen gas are the tropics and warm climates, where a sky, seldom clouded, permits the glowing rays of the sun to shine upon an immeasurably luxuriant vegetation. The temperate and cold zones, where artificial warmth must replace deficient heat of the sun, produce, on the contrary, carbonic acid in superabundance, which is expended in the nutrition of the tropical plants. 'The same stream of air, which moves by the revolution of the earth from the equator to the poles, brings to us, in its passage from the equator, the oxygen generated there, and carries away the carbonic acid formed during our winter.

accordingly be formed 7686 grammes of carbonate of lime, which con. tain $4325^{\circ} 6$ grammes of carbonic acid. The weight of one cubic decimetre of carbonic acid being calculated at two grammes, (more accurately 1.97978,) the above-mentioned surface must absorb in four days $2 \cdot 163$ cubic metres of carbonic acid. 2500 square metres (one Hessian acre) would absorb, under a similar treatment, $51 \frac{1}{2}$ cubic metres $=1818$ cubic feet of carbonic acid in four days. In 200 days it would absorb 2575 cubic metres $=904,401$ cubic feet, which contain 11,353 lbs. of carbonic acid, of which 3304 lbs. are carbon, a quantity three times as great as that which is assimilated by the leaves and roots growing upon the same space. - L. L.

* Plants that grow in the dark, are well known to be colorless. This is seen in the blanching of celery (etiolation), the earth is heaped around the stalks to exclude the light. 
The experiments of De Saussure have proved, that the upper strata of the air contain more carbonic acid than the lower, which are in contact with plants; and that the quantity is greater by night than by day, when it undergoes decomposition.

Plants thus improve the air, by the removal of carbonic acid, and by the renewal of oxygen, which is immediately applied to the use of man and animals. The horizontal currents of the atmosphere bring with them as much as they carry away, and the interchange of air between the upper and lower strata, which their difference of temperature causes, is extremely trifling when compared with the horizontal movements of the winds. Thus vegetable culture heightens the healthy state of a country, and a previously healthy country would be rendered quite uninhabitable by the cessation of all cultivation.

The various layers of wood and mineral coal, as well as peat, form the remains of a primeval vegetation. 'The carbon which they contain must have been originally in the atmosphere as carbonic acid, in which form it was assimilated by the plants which constitute these formations. It follows from this, that the atmosphere must be richer in oxygen at the present time than in former periods of the earth's history. The increase must be exactly proportional to the quantity of carbon and hydrogen contained in these carboniferous deposits. Thus, during the formation of 353 cubic feet of Newcastle splint-coal, the atmosphere must have received 643 cubic feet of oxygen produced from the carbonic acid assimilated, and also 158 cubic feet of the same gas resulting from the decomposition of water. In former ages, therefore, the atmosphere must have contained less oxygen, but a much larger proportion of carbonic acid, than it does at the present time, a circumstance which accounts for the richness and luxuriance of the earlier vegetation.

But a certain period must have arrived in which the quantity of carbonic acid contained in the air 
experienced neither increase nor diminution in any appreciable quantity. For if it received an additional quantity to its usual proportion, an increased vegetation would be the natural consequence, and the excess would thus be speedily removed. And, on the other hand, if the gas was less than the normal quantity, the progress of vegetation would be retarded, and the proportion would soon attain its proper standard.

The most important function in the life of plants, or, in other words, in their assimilation of carbon, is the separation, we might almost say, the generation of oxygen. No matter can be considered as nutritious, or as necessary to the growth of plants, which possesses a composition either similar to or identical with theirs, and the assimilation of which, therefore, could take place without exercising this function. The reverse is the case in the nutrition of animals. Hence such substances as sugar, starch, and gum, which are themselves products of plants, cannot be adapted for assimilation. And this is rendered certain by the experiments of vegetable physiologists, who have shown that aqueous solutions of these bodies are imbibed by the roots of plants, and carried to all parts of their structure, but are not assimilated; they cannot therefore be employed in their nutrition. We could scarcely conceive a form more convenient for assimilation than that of gum, starch, and sugar, for they all contain the elements of woody fibre, and nearly in the same proportions.

In the second part of the work we shall adduce satisfactory proofs that decayed woody fibre (humus) contains carbon and the elements of water, without an excess of oxygen; its composition differing from that of woody fibre in its being richer in carbon.

Misled by this simplicity in its constitution, physiologists found no difficulty in discovering the mode. of the formation of woody fibre; for they say, ${ }^{*}$ hu- 
mus has only to enter into combination with water, in order to effect the formation of woody fibre, and other substances similarly composed, such as sugar, starch, and gum. But they forget, that their own experiments have sufficiently demonstrated the inaptitude of these substances for assimilation.

All the erroneous opinions concerning the modus operandi of humus have their origin in the false notions entertained respecting the most important vital functions of plants; analogy, that fertile source of error, having, unfortunately, led to the very unapt comparison of the vital functions of plants with those of animals.

Substances, such as sugar, starch, \&c., which contain carbon and the elements of water, are products of the life of plants which live only whilst they generate them. The same may be said of humus, for it can be formed in plants like the former substances. Smithson, Jameson, and Thomson, found that the black excretions of unhealthy elms, oaks, and horse-chesnuts, consisted of humic acid in combination with alkalies. Berzelius detected similar products in the bark of most trees. Now, can it be supposed that the diseased organs of a plant possess the power of generating the matter to which its sustenance and vigor are ascribed?

How does it happen, it may be asked, that the absorption of carbon from the atmosphere by plants is doubted by all botanists and vegetable physiologists, and that by the greater number the purification of the air by mearis of them is wholly denied?

The action of plants on the air in the absence of light, that is during night, has been much misconceived by botanists, and from this we may trace most of the errors which abound in the greater part of their writings. The experiments of Ingenhouss were in a great degree the cause of this uncertainty of opinion regarding the influence of plants in purifying the air. His observation, that green plants. emit carbonic acid in the dark, led De Saussure and 
Grischow to new investigations, by which they ascertained, that under such conditions plants do really absorb oxygen and emit carbonic acid; but that the whole volume of air undergoes diminution at the same time. From the latter fact it follows, that the quantity of oxygen gas absorbed is greater than the volume of carbonic acid separated; for, if this were not the case, no diminution could occur. These facts cannot be doubted, but the views based on them have been so false, that nothing, except the total want of observation and the utmost ignorance of the chemical relations of plants to the atmosphere, can account for their adoption.

It is known that nitrogen, hydrogen; and a number of other gases, exercise a peculiar, and in general an injurious influence upon living plants. Is it, then, probable, that oxygen, one of the most energetic agents in nature, should remain without influence on plants when one of their peculiar processes of assimilation has ceased?

It is true that the decomposition of carbonic acid is arrested by absence of light. But then, namely, at night, a true chemical process commences, in consequence of the action of the oxygen in the air, upon the organic substances composing the leaves, blossoms, and fruit. This process is not at all connected 'with the life of the vegetable organism, because it goes on in a dead plant exactly as in a living one.

The substances composing the leaves of different plants being known, it is a matter of the greatest ease and certainty to calculate which of them, during life, should absorb most oxygen by chemical action when the influence of light is withdrawn.

The leaves and green parts of all plants containing volatile oils or volatile constituents in general, which change into resin by the absorption of oxygen, should absorb more than other parts which are free from such substances. Those leaves, also, which contain either the constituents of nut-galls, or com- 
pounds in which nitrogen is present, ought to absorb more oxygen than those which do not contain such matters. The correctness of these inferences has been distinctly proved by the observations of $\mathrm{De}$ Saussure; for, whilst the tasteless leaves of the Agave americana absorb only 0.3 of their volume of oxygen in the dark, during 24 hours, the leaves of the Pinus Abies, which contain volatile and resinous oils, absorb 10 times, those of the Quercus Robur containing tannic acid 14 times, and the balmy leaves of the Populus alba 21 times that quantity. This chemical action is shown very plainly, also, in the leaves of the Cotyledon calycinum, the Cacalia ficoides, and others; for they are sour like sorrel in the morning, tasteless at noon, and bitter in the evening. The formation of acids is effected during the night by a true process of oxidation: these are deprived of their acid properties during the day and evening, and are changed by separation of a part of their oxygen into compounds containing oxygen and hydrogen, either in the same proportions as in water, or even with an excess of hydrogen, which is the composition of all tasteless and bitter substances.

Indeed the quantity of oxygen absorbed could be estimated pretty nearly by the different periods which the green leaves of plants require to undergo alteration in color, by the influence of the atmosphere. Those which continue longest green will abstract less oxygen from the air in an equal space of time, than those, the constituent parts of which suffer a more rapid change. It is found, for example, that the leaves of the Ilex aquifolium, distinguished by the durability of their color, absorb only 0.86 of their volume of oxygen gas in the same time that the leaves of the poplar absorb 8, and those of the beech $9 \frac{1}{2}$ times their volume; both the beech and poplar being remarkable for the rapidity and ease with which the color of their leaves changes.

When the green leaves of the poplar, the beech, the oak, or the holly, are dried under the air-pump, 
with exclusion of light, then moistened with water, and placed under a glass globe filled with oxygen, they are found to absorb that gas in proportion as they change in color. The chemical nature of this process is thus completely established. The diminution of the gas which occurs can only be owing to the union of a large proportion of oxygen with those substances which are already in the state of oxides, or to the oxidation of the hydrogen in those vegetable compounds which contain it in excess. "The fallen brown or yellow leaves of the oak contain no longer tannin, and those of the poplar no balsamic constituents.

The property which green leaves possess of absorbing oxygen belongs also to fresh wood, whether taken from a twig or from the interior of the trunk of a tree. When fine chips of such wood are placed in a moist condition under a jar filled with oxygen, the gas is seen to diminish in volume. But wood, dried by exposure to the atmosphere and then moistened, converts the oxygen into carbonic acid, without change of volume; fresh wood, therefore, absorbs most oxygen.

MM. Petersen and Schödler have shown, by the careful elementary analysis of 24 different kinds of wood, that they contain carbon and the elements of water, with the addition of a certain quantity of hydrogen. Oak wood, recently taken from the tree, and dried at $100^{\circ}$ C. $\left(212^{\circ}\right.$ F.), contains 49.432 carbon, 6.069 hydrogen, and 44.499 oxygen.

The proportion of hydrogen which is necessary to combine with 44.498 oxygen in order to form water, is $\frac{1}{8}$ of this quantity, namely, 5.56 ; it is evident, therefore, that oak wood contains $\frac{1}{12}$ more hydrogen than corresponds to this proportion. In Pinus Larix, $\boldsymbol{P}$. Abies, and $\boldsymbol{P}$. picea, the excess of hydrogen amounts to $\frac{1}{7}$, and in Tilia europaea to $\frac{1}{5}$. The quantity of hydrogen stands in some relation to the specific weight of the wood; the lighter kinds of wood contain more of it than the heavier. In ebony 
wood (Diospyros Ebenum) the oxygen and hydrogen are in exactly the same proportion as in water.

The difference between the composition of the varieties of wood, and that of simple woody fibre, depends, unquestionably, upon the presence of constituents, in part soluble, and in part insoluble, such as resin and other matters, which contain a large proportion of hydrogen: the hydrogen of such substances being in the analysis of the various woods superadded to that of the true woody fibre.

It has previously been mentioned that mouldering oak wood contains carbon and the elements of water, without any excess of hydrogen. But the proportions of its constituents must necessarily have been different, if the volume of the air had not changed during its decay, because the proportion of hydrogen in those component substances of the wood which contained it in excess is here diminished, and this diminution could only be effected by an absorption of oxygen, and consequent formation of water.

Most vegetable physiologists have connected the emission of carbonic acid during the night with the absorption of oxygen from the atmosphere, and have considered these actions as a true process of respiration in plants, similar to that of animals, and like it, having for its result the separation of carbon from some of their constituents. This opinion has a very weak and unstable foundation.

The carbonic acid, which has been absorbed by the leaves and by the roots, together with water, ceases to be decomposed on the departure of daylight; it is dissolved in the juices which pervade all parts of the plant, and escapes every moment through the leaves in quantity corresponding to that of the water which evaporates.

A soil in which plants vegetate vigorously, contains a certain quantity of moisture which is indispensably necessary to their existence. Carbonic acid, likewise, is always present in such a soil, whether it has been abstracted from the air or has 
been generated by the decay of vegetable matter. Rain and well water, and also that from other sources, invariably contains carbonic acid.-Plants during their life constantly possess the power of absorbing by their roots moisture, and, along with it, air and carbonic acid. Is it, therefore, surprising that the carbonic acid should be returned unchanged to the atmosphere, along with water, when light (the cause of the fixation of its carbon) is absent?

Neither this emission of carbonic acid nor the absorption of oxygen has any connexion with the process of assimilation; nor have they the slightest relation to one another; the one is a purely mechanical, the other a purely chemical process. A cotton wick, inclosed in a lamp, which contains a liquid saturated with carbonic acid, acts exactly in the same manner as a living plant in the night. Water and carbonic acid are sucked up by capillary attraction, and both evaporate from the exterior part of the wick.

Plants which live in a soil containing humus exhale much more carbonic acid during the night than those which grow in dry situations; they also yield more in rainy than in dry weather. These facts point out to us the cause of the numerous contradictory observations, which have been made with respect to the change impressed upon the air by living plants, both in darkness and in common daylight, but which are unworthy of consideration, as they do not assist in the solution of the main question.

There are other facts which prove in a decisive manner that plants yield more oxygen to the atmosphere than they extract from it; these proofs, however, are to be drawn with certainty only from plants which live under water.

When pools and ditches, the bottoms of which are covered with growing plants, freeze upon their surface in winter, so that the water is completely excluded from the atmosphere by a clear stratum of ice, small bubbles of gas are observed to escape, con- 
tinually, during the day, from the points of the leaves and twigs. 'These bubbles are seen most distinctly when the rays of the sun fall upon the ice; they are very small at first, but collect under the ice and form larger bubbles. They consist of pure oxygen gas. Neither during the night, nor during the day when the sun does not shine, are they observed to diminish in quantity. The source of this oxygen is the carbonic acid dissolved in the water, which is absorbed by the plants, but is again supplied to the water, by the decay of vegetable substances contained in the soil. If these plants absorb oxygen during the night, it can be in no greater quantity than that which the surrounding water holds in solution, for the gas, which has been exhaled, is not again absorbed. The action of water-plants cannot be supposed to form an exception to a great law of nature, and the less so,'as the different action of aërial plants upon the atmosphere is very easily explained.

The opinion is not new, that the carbonic acid of the air serves for the nutriment of plants, and that its carbon is assimilated by them; it has been admitted, defended, and argued for, by the soundest and most intelligent natural philosophers, namely, by Priestley, Sennebier, De Saussure, and even by Ingenhouss himself. There scarcely exists a theory in natural science, in favor of which there are more clear and decisive arguments. How, then, are we to account for its not being received in its full extent by most other physiologists, for its being even disputed by many, and considered by a few as quite refuted?

All this is due to two causes, which we shall now consider.

One is, that in botany the talent and labor of inquirers has been wholly spent in the examination of form and structure: chemistry and physics have not been allowed to sit in council upon the explanation of the most simple processes; their experience and their laws have not been employed, though the most 
powerful means of help in the acquirement of true knowledge. They have not been used, because their study has been neglected.

All discoveries in physics and in chemistry, all explanations of chemists, must remain without fruit and useless, because, even to the great leaders in physiology, carbonic acid, ammonia, acids, and bases, are sounds without meaning, words without sense, terms of an unknown language, which awaken no thoughts and no associations. They treat these sciences like the vulgar, who despise a foreign literature in exact proportion to their ignorance of it; since even when they have had some acquaintance with them, they have not understood their spirit and application.

Physiologists reject the aid of chemistry in their inquiry into the secrets of vitality, although it alone could guide them in the true path; they reject chemistry, because in its pursuit of knowledge it destroys the subjects of its investigation; but they forget that the knife of the anatomist must dismember the body, and destroy its organs, if an account is to be given of their form, structure, and functions.

When pure potato starch is dissolved in nitric acid, a ring of the finest wax remains. What can be opposed to the conclusion of the chemist, that each grain of starch consists of concentric layers of wax and amylin, which thus mutually protect each other against the action of water and ether? Can results of this kind, which illustrate so completely both the nature and properties of bodies, be attained by the microscope? Is it possible to make the gluten in a piece of bread visible in all its connexions and ramifications? It is impossible by means of instruments; but if the piece of bread is placed in a lukewarm decoction of malt, the starch, and the substance called dextrine, ${ }^{*}$ are seen to dissolve like

* According to Raspail, starch consists of vesicles inclosing within them a fluid resembling gum. Starch may be put in cold water without being dissolved; but, when placed in hot water, these spherules 
sugar in water, and, at last, nothing remains except the gluten, in the form of a spongy mass, the minute pores of which can be seen only by a microscope.

Chemistry offers innumerable resources of this kind which are of the greatest use in an inquiry into the nature of the organs of plants; but they are not used, because the need of them is not felt. The most important organs of animals and their functions are known, although they may not be visible to the naked eye. But in vegetable physiology, a leaf is in every case regarded merely as a leaf, notwithstanding that leaves generating oil of turpentine or oil of lemons must possess a different nature from those in which oxalic acid is formed. Vitality, in its peculiar operations, makes use of a special apparatus for each function of an organ. A rose-twig engrafted upon a lemon-tree does not bring forth lemons, but roses. Vegetable physiologists in the study of their science have not directed their attention to that part of it which is most worthy of investigation.

The second cause of the incredulity with which physiologists view the theory of the nutrition of plants by the carbonic acid of the atmosphere is, that the art of experimenting is not known in physiology, it being an art which can be learned accurately only in the chemical laboratory. Nature speaks to us in a peculiar language, in the language of phenomena; she answers at all times the questions which are put to her; and such questions are experiments. An experiment is the expression of a thought: we are near the truth when the phenomenon elicited by the experiment corresponds to the thought; while

burst, and allow the escape of the liquid. This liquid is the dextrine of Biot, so called because it possesses the property of turning the plane of the polarization of light to the right hand. It is white, insipid, transparent in thin flakes and gummy. At $230^{\circ} \mathrm{F}$. it becomes brown and acquires the flavor of toasted bread. It is much employed by the French pastry cooks and confectioners; being reduced to powder it may be introduced into all kinds of pastries, bread, chocolate, \&c. For its preparation, \&c., see URE's Dictionary of Arts and Manufactures, and WEBSTER's Chemistry, 510. 
the opposite result shows that the question was falsely stated, and that the conception was erroneous.

The critical repetition of another's experiments must be viewed as a criticism of his opinions; if the result of the criticism be merely negative, if it do not suggest more correct ideas in the place of those which it is intended to refute, it should be disregarded; because the worse experimenter the critic is, the greater will be the discrepancy between the results he obtains and the views proposed by the other.

It is too much forgotten by physiologists, that their duty really is not to refute the experiments of others, nor to show that they are erroneous, but to discover truth, and that alone. It is startling, when we reflect that all the time and energy of a multitude of persons of genius, talent, and knowledge, are expended in endeavors to demonstrate each other's errors.

The question whether carbonic acid is the food of plants or not has been made the subject of experiments with perfect zeal and good faith; the results have been opposed to that view. But how was the inquiry instituted?

The seeds of balsamines, beans, cresses, and gourds, were sown in pure Carrara marble, and sprinkled with water containing carbonic acid. The seeds sprang, but the plants did not attain to the development of the third small leaf. In other cases, they allowed the water to penetrate the marble from below, yet, in spite of this, they died. It is worthy of observation, that they lived longer with pure distilled water than with that impregnated with carbonic acid; but still, in this case also, they eventually perished. Other experimenters sowed seeds of plants in flowers of sulphur and sulphate of barytes, and tried to nourish them with carbonic acid, but without success.

Such experiments have been considered as positive proofs, that carbonic acid will not nourish plants; 
but the manner in which they were instituted is opposed to all rules of philosophical inquiry, and to all the laws of chemistry.

Many conditions are necessary for the life of plants; those of each genus require special conditions; and should but one of these be wanting, although the rest be supplied, the plants will not be brought to maturity. The organs of a plant, as well as those of an animal, contain substances of the most different kinds; some are formed solely of carbon and the elements of water, others contain nitrogen, and in all plants we find metallic oxides in the state of salts. The food which can serve for the production of all the organs of a plant, must necessarily contain all its elements. These most essential of all the chemical qualities of nutriment may be united in one substance, or they may exist separately in several; in which case, the one contains what is wanting in the other. Dogs die although fed with jelly, a substance which contains nitrogen; they cannot live upon white bread, sugar, or starch, if these are given as food, to the exclusion of all other substances. Can it be concluded from this, that these substances contain no elements suited for assimilation? Certainly not.

Vitality is the power which each organ possesses of constantly reproducing itself; for this it requires a supply of substances which contain the constituent elements of its own substance, and are capable of undergoing transformation. All the organs together cannot generate a single element, carbon, nitrogen, or a metallic oxide.

When the quantity of the food is too great, or is not capable of undergoing the necessary transformation, or exerts any peculiar chemical action, the organ itself is subjected to. a change: all poisons act in this manner. The most nutritious substances may cause death. In experiments such as those described above, every condition of nutrition should be considered. Besides those matters which form their principal constituent parts, both animals and plants 
require others, the peculiar functions of which are unknown. These are inorganic substances, such as common salt, the total want of which is in animals inevitably productive of death. Plants, for the same reason, cannot live unless supplied with certain metallic compounds.

If we knew with certainty that there existed a substance capable, alone, of nourishing a plant and of bringing it to maturity, we might be led to a knowledge of the conditions necessary to the life of all plants, by studying its characters and composition. If humus were such a substance, it would have precisely the same value as the only single food which nature has produced for animal organization, namely, milk. (Prout.) The constituents of milk are cheese or caseine, a compound containing nitrogen in large proportion; butter, in which hydrogen abounds; and sugar of milk, a substance with a large quantity of hydrogen and oxygen in the same proportion as in water. It also contains in solution, lactate of soda, phosphate of lime, and common salt; and a peculiar aromatic product exists in the butter, called butyric acid. The knowledge of the composition of milk is a key to the conditions necessary for the purposes of nutrition of all animals.

All substances which are adequate to the nourishment of animals contain those materials united, though not always in the same form; nor can any one be wanting for a certain space of time, without a marked effect on the health being produced. The employment of a substance as food presupposes a knowledge of its capacity of assimilation, and of the conditions under which this takes place.

A carnivorous animal dies in the vacuum of an air-pump, even though supplied with a superabundance of food; it dies in the air, if the demands of its stomach are not satisfied; and it dies in pure oxygen gas, however lavishly nourishment be given to it. Is it hence to be concluded, that neither flesh, 
nor air, nor oxygen, is fitted to support life ? Certainly not.

From the pedestal of the Trajan column at Rome we might chisel out each single piece of stone, if upon the extraction of the second we replaced the first. But could we conclude from this that the column was suspended in the air, and not supported by a single piece of its foundation? Assuredly not. Yet the strongest proof would have been given that each portion of the pedestal could be removed, without the downfall of the column.

Animal and vegetable physiologists, however, come to such conclusions with respect to the process of assimilation. They institute experiments, without being acquainted with the circumstances necessary for the continuance of life, - with the qualities and proper nutriment of the animal or plant on which they operate, - or with the nature and chemical constitution of its organs. These experiments are considered by them as convincing proofs, whilst they are fitted only to awaken pity.

Is it possible to bring a plant to maturity by means of carbonic acid and water, without the aid of some substance containing nitrogen, which is an essential constituent of the sap, and indispensable for its production? Must the plant not die, however abundant the supply of carbonic acid may be, as soon as the first small leaves have exhausted the nitrogen contained in the seeds?

Can a plant be expected to grow in Carrara marble, even when an azotized substance is supplied to it, if the marble be sprinkled with an aqueous solution of carbonic acid, which dissolves the lime and forms bicarbonate of lime? A plant of the family of the Plumbaginea, upon the leaves of which fine hornlike, or scaly processes of crystallized carbonate of lime are formed, might perháps' attain maturity under such circumstances; but these experiments. are only sufficient to prove, that cresses, gourds, and balsamines, cannot be nourished by bicarbonate of 
lime, in the absence of matter containing nitrogen. We may, indeed, conclude, that the salt of lime acts as a poison, since the development of plants will advance further in pure water, when lime and carbonic acid are not used.

Moist flowers of sulphur attract oxygen from the atmosphere, and become acid. Is it possible that a plant can grow and flourish in presence of free sulphuric acid, with no other nourishment than carbonic acid? It is true, the quantity of sulphuric acid formed thus in hours, or in days, may be small, but the property of each particle of the sulphur to absorb oxygen and retain it, is present every moment.

When it is known that plants require moisture, carbonic acid, and air, should we choose, as the soil for experiments on their growth, sulphate of barytes, which, from its nature and specific gravity, completely prevents the access of air.

All these experiments are valueless for the decision of any question. It is absurd to take for them any soil, at mere hazard, so long as we are ignorant of the functions performed in plants by those inorganic substances which are apparently foreign to them. It is quite impossible to mature a plant of the family of the Graminea, or of the Equisetacea, the solid framework of which contains silicate of potash, without silicic acid and potash, or a plant of the genus Oxalis without potash, or'saline plants such as the saltworts (Salsola and Salicornia) without chloride of sodium, or at least some salt of similar properties. All seeds of the Graminea contain phosphate of magnesia; the solid parts of the roots of the althae contain more phosphate of lime than woody fibre. Are these substances merely accidentally present? A plant should not be chosen for experiment, when the matter which it requires for its assimilation is not well known.

What value, now, can be attached to experiments in which all those matters which a plant requires in the process of assimilation, besides its mere nutri- 
ment, have been excluded with the greatest care? Can the laws of life be investigated in an organized being which is diseased or dying?

The mere observation of a wood or meadow is infinitely better adapted to decide so simple a question than all the trivial experiments under a glass globe; the only difference is, that instead of one plant there are thousands. When we are acquainted with the nature of a single cubic inch of their soil, and know the composition of the air and rain-water, we are in possession of all the conditions necessary to their life. The source of the different elements entering into the composition of plants cannot possibly escape us, if we know in what form they take up their nourishment, and compare its composition with that of the vegetable substances which compose their structure.

All these questions will now be examined and discussed. It has been already shown, that the carbon of plants is derived from the atmosphere: it still remains for us to inquire, what power is exerted on vegetation by the humus of the soil and the inorganic constituents of plants, and also to trace the sources of their nitrogen.

\section{CHAPTER III.}

ON THE ORIGIN AND ACTION OF HUMUS.

IT will be shown in the second part of this work, that all plants and vegetable structures undergo two processes of decomposition after death. One of these is named fermentation; the other, putrefaction, decay, or eremacausis.*

* The word cremacausis was proposed by the author some time since, in order to explain the true nature of decay; it is compounded from

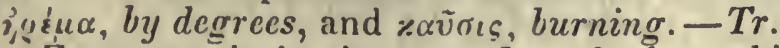

Eremacausis is the act of gradual combination of the combustible 
It will likewise be shown, that decay is a slow process of combustion, - a process, therefore, in which the combustible parts of a plant unite with the oxygen of the atmosphere.

The decay of woody fibre (the principal constituent of all plants) is accompanied by a phenomenon of a peculiar kind. This substance, in contact with air or oxygen gas, converts the latter into an equal volume of carbonic acid, and its decay ceases upon the disappearance of the oxygen. If the carbonic acid is removed, and oxygen replaced, its decay recommences, that is, it again converts oxygen into carbonic acid. Woody fibre consists of carbon and the elements of water; and if we judge only from the products formed during its decomposition, and from those formed by pure charcoal, burned at a high temperature, we might conclude that the causes were the same in both: the decay of woody fibre proceeds, therefore, as if no hydrogen or oxygen entered into its composition.*

A very long time is required for the completion of this process of combustion, and the presence of water is necessary for its maintenance; alkalies promote it, but acids retard it; all antiseptic sub-

elements of a body with the oxygen of the air; a slow combustion or oxidation.

The conversion of wood into humus, the formation of acetic acid out of alcohol, nitrification, and numerous other processes, are of this nature. Vegetable juices of every kind, parts of animal and vegetable substances, moist sawdust, blood, \&c., cannot be exposed to the air, without suffering immediately a progressive change of color and properties, during which oxygen is absorbed. These changes do not take place when water is excluded, or when the substances are exposed to the temperature of $32^{\circ}$, and different bodies require different degrees of heat, in order to effect the absorption of oxygen, and, consequently, their eremacausis. The property of suffering this change is possessed in the highest degree by substances which contain nitrogen. - Liebig. Org. Chem. Part 2d.

* In the Appendix to the Third Report of the Agriculture of Massachusetts, 1840, Dr. S. L. Dana adduces the following example, to show that even a moist plant will not decay, if air is excluded. A piece of a white birch tree was taken from a depth of twenty-five feet below the surface, in Lowell. "It must have been inhumed there probably before the creation of man, yet this most perishable of all wood is nearly as sound as if cut from the forest last fall." 
stances, such as sulphurous acid, the mercurial salts, empyreumatic oils, \&c., cause its complete cessation.

Woody fibre in a state of decay is the substance called humus.*

The property of woody fibre to convert surrounding oxygen gas into carbonic acid diminishes in proportion as its decáy advances, and at last a certain quantity of a brown coaly-looking substance remains, in which this property is entirely wanting. This substance is called mould; it is the product of the complete decay of woody fibre. Mould constitutes the principal part of all the strata of brown coal and peat.

Humus acts in the same manner in a soil permeable to air as in the air itself ; it is a continued source of carbonic acid, which it emits very slowly. An atmosphere of carbonic acid, formed at the expense of the oxygen of the air, surrounds every particle of decaying humus. The cultivation of land, by tilling and loosening the soil, causes a free and unobstructed access of air. An atmosphere of carbonic acid is therefore contained in every fertile soil, and is the first and most important food for the young plants which grow in it.

In spring, when those organs of plants are absent which nature has appointed for the assumption of nourishment from the atmosphere, the component substance of the seeds is exclusively employed in the formation of the roots. Each new radicle fibril which a plant acquires may be regarded as constituting at the same time a mouth, a lung, and a stomach. The roots perform the functions of the leaves from the first moment of their formation: they extract from the soil their proper nutriment, namely, the carbonic acid generated by the humus.

By loosening the soil which surrounds young plants, we favor the access of air, and the formation

* The humic acid of chemists is a product of the decomposition of humus by alkalies; it does not exist in the humus of vegetable physiologists. $-L$. 
of carbonic acid; and, on the other hand, the quantity of their food is diminished by every difficulty which opposes the renewal of air. A plant itself effects this change of air at a certain period of its growth. The carbonic acid, which protects the undecayed humus from further change, is absorbed and taken away by the fine fibres of the roots, and by the roots themselves; this is replaced by atmospheric air, by which process the decay is renewed, and a fresh portion of carbonic acid formed. A plant at this time receives its food both by the roots and by the organs above ground, and advances. rapidly to maturity.

When a plant is quite matured, and when the organs by which it obtains food from the atmosphere are formed, the carbonic acid of the soil is no further required.

Deficiency of moisture in the soil, or its complete dryness, does not now check the growth of a plant, provided it receives from the dew and the atmosphere as much as is requisite for the process of assimilation. During the heat of summer it derives its carbon exclusively from the atmosphere.

We do not know what height and strength nature has allotted to plants; we are acquainted only with the size which they usually attain. Oaks are shown, both in London and Amsterdam, as remarkable curiosities, which have been reared by Chinese gardeners, and are only one foot and a half in height, although their trunks, barks, leaves, branches; and whole habitus, evince a venerable age. The small parsnep grown at Teltow, ${ }^{*}$ when placed in a soil which yields as much nourishment as it can take up, increases to several pounds in weight.

The size of a plant is proportional to the surface of the organs. which are destined to convey food to it.

* Teltow is a village near Berlin, where small parsneps are cultivated in a sandy soil; they are much esteemed, and weigh rarely above one ounce. $-L$. 
A plant gains another mouth and stomach with every new fibre of root, and every new leaf.

The power which roots possess of taking up nourishment does not cease as long as nutriment is present. When the food of a plant is in greater quantity than its organs require for their own perfect development, the superfluous nutriment is not returned to the soil, but is employed in the formation of new organs. At the side of a cell, already formed, another cell arises; at the side of a twig and leaf, a new twig and a new leaf are developed. These new parts could not have been formed had there not been an excess of nourishment. The sugar and mucilage produced in the seeds, form the nutriment of the young plants, and disappear during the development of the buds, green sprouts, and leaves.

The power of absorbing nutriment from the atmosphere, with which the leaves of plants are endowed, being proportionate to the extent of their surface, every increase in the size and number of these parts is necessarily attended with an increase of nutritive power, and a consequent further development of new leaves and branches. Leaves, twigs, and branches, when completely matured, as they do not become larger, do not need food for their support." For their existence as organs, they require only the means-necessary for the performance of the special functions to which they are destined by nature; they do not exist on their own account.

We know that the functions of the leaves and other green parts of plants are to absorb carbonic acid, and with the aid of light and moisture, to appropriate its carbon. These processes are continually in operation; they commence with the first formation of the leaves, and do not cease with their perfect development. But the new products arising from this continued assimilation are no longer employed by the perfect leaves in their own increase : they serve for the formation of woody fibre, and all the solid matters of similar composition. The leaves: 
now produce sugar, amylin or starch; and acids, which were previously formed by the roots, when they were necessary for the development of the stem, buds, leaves, and branches of the plant.

The organs of assimilation, at this period of their life, receive more nourishment from the atmosphere than they employ in their own sustenance; and when the formation of the woody substance has advanced to a certain extent, the expenditure of the nutriment, the supply of which still remains the same, takes a new direction, and blossoms are produced. The functions of the leaves of most plants cease upon the ripening of their fruit, because the products of their action are no longer needed. They now yield to the chemical influence of the oxygen of the air, generally suffer a change in color, and fall off.

A peculiar "transformation" of the matters contained in all plants takes place in the period between blossoming and the ripening of the fruit; new compounds are produced, which furnish constituents of the blossoms, fruit, and seed. An organic chemical "transformation" is the separation of the elements of one or several combinations, and their reunion into two or several others, which contain the same number of elements, either grouped in another manner, or in different proportions. Of two compounds formed in consequence of such a change, one remains as a component part of the blossom or fruit, while the other is separated by the roots in the form of excrementitious matter. No process of nutrition can be conceived to subsist in animals or vegetables, without a separation of effete matters. We know, indeed, that an organized body cannot generate substances, but can only change the mode of their combination, and that its sustenance and reproduction depend upon the chemical transformation of the matters which are employed as its nutriment, and which contain its own constituent elements.

Whatever we regard as the cause of these transformations, whether the Vital Principle, Increase of 
Temperature, Light, Galvanism, or any other influence, the act of transformation is a purely chemical process. Combination and Decomposition can take place only when the elements are disposed to these changes. That which chemists name affinity indicates only the degree in which they possess this disposition. It will be shown, when considering the processes of fermentation and putrefaction, that every disturbance of the mutual attraction subsisting between the elements of a body gives rise to a transformation. The elements arrange themselves according to the degrees of their reciprocal attraction into new combinations, which are incapable of further change under the same conditions.

The products of these transformations vary with their causes, that is, with the different conditions on which their production depended; and are as innumerable as these conditions themselves. The chemical character of an acid, for example, is its unceasing disposition to saturation by means of a base; * this disposition differs in intensity in different acids; but when it is satisfied, the acid character entirely disappears. The chemical character of a base is exactly the reverse of this, but both an acid and a base, notwithstanding the great difference in their

* Liebig applies the term base to compounds which unite with acids and neutralize their characters. The product is a salt. When the characters of both acids and bases disappear the compound is neutral.

Some acids contain oxygen, others hydrogen. Several metals form acids with oxygen; but the greater number of metallic oxides, are, in their relations, totally different from the acids. They form compounds, which, for the most part, are insoluble in water; those soluble in water have an alkaline taste, and possess the property of restoring the blue color of vegetables, which have been reddened by acids. These also change many vegetable yellows to red or brown. The alkalies are soluble bases. Many salts redden vegetable blues, and others again restore the blue color of vegetables reddened by acids; in the first instance, the salt possesses an acid, and in the latter an alkaline, reaction.

A simple body, which is capable of forming either an acid or a base, is termed a radical; a compound radical consists of two or three simple radicals, and comports itself in a similar manner to the simple radicals; that is, it is capable of forming acids and bases. 
properties, effect, in most cases, the same kind of transformations.

Hydrocyanic acid (prussic acid)* and water contain the elements of carbonic acid, ammonia, urea, cyanuric acid, cyanilic acid, oxalic acid, formic acid, melam, ammelin, melamin, azulmin, mellon, hydromellonic acid, allantoin, \&॰c. $\dagger$ It is well known, that all these very different substances can be obtained

* Cyanogen is considered by Liebig as a compound base, and as such uniting with oxygen, hydrogen, and most other nonmetallic elements and with the metals. Cyanogen gas, or bicarburet of nitrogen, is a compound of nitrogen and carbon, and was named from its affording a blue color and being an ingredient of Prussian blue. For the method of obtaining it, \&c., see WEBSTER's Chemistry, 3d edition, p. 219.

With hydrogen it constitutes hydrocyanic acid.

+ Carbonic acid is a gaseous compound of 1 equivalent of carbon, and 2 equivalents of oxygen, represented thus, $C+20$ or $\ddot{\mathrm{C}}$, the two dots denoting the two of oxygen.

Ammonia consists of 3 equivalents of hydrogen, and 1 equivalent of nitrogen, represented thus, $\mathrm{N}+3 \mathrm{H}$, or $\mathrm{NH}_{3}$.

Urea contains the elements of cyanate of ammonia $\left(\mathrm{NH}_{4} \mathrm{O}+\mathrm{C}_{4} \mathrm{NO}\right)$, and exists in urine, from which it is obtained in colorless, transparent crystals.

Cyanuric acid is a product of the decomposition of chloride of cyanogen, of urea, \&c. It is called a tribasic acid, and its hydrate is thus represented, $\mathrm{Cy}_{3} \mathrm{O}_{3}+3 \mathrm{HO}$.

Oxalic ucid is a solid acid. obtained from several plants, particularly of the genera oxalis, rumex, \&c. combined with potassa in roots, and with lime in several kinds of liclens. Oxalate of linie is found in urinary calculi. It is represented thus, $2 \mathrm{CO}+\mathrm{O}$ ( 2 equivalents of carbonic oxide +1 oxygen). The so-called Essential salt of lemons is a binoxalate of potash. It is poisonous.

Formic acid, obtained from ants, hence its name. It is now obtained from sugar and other vegetable substances. Represented by $\mathrm{C}_{2} \mathrm{HO}_{3}$.

Melam is a compound of $\mathrm{C}_{12} \mathrm{~N}_{11} \mathrm{H}_{9}$; it is a white powder insoluble in water, and, by the action of acids, converted into cyanuric acid and ammonia.

Ammelin, a saline base, represented thus, $\mathrm{C}_{6} \mathrm{~N}_{5} \mathrm{H}_{5} \mathrm{O}_{2}$, a product of the decomposition of melam by acids and alkalies.

Melamin, a saline base, product of the decomposition of melam, $\mathrm{C}_{6} \mathrm{~N}_{6} \mathrm{H}_{6}$. Decomposed by acids into ammonia and ammelid or ammelin.

Azulmen, the base of azulmic acid, obtained by the decomposition of cyanogen. The acid is $\mathrm{C}_{8} \mathrm{H}_{4} \mathrm{~N}_{4} \mathrm{O}_{4}$.

Mellon, a compound base, a yellow powder. Decomposed into 3 volumes cyanogen and 1 volume nitrogen gas. $\mathrm{C}_{6} \mathrm{~N}_{4}$.

Hydromellonic acid is $\mathrm{C}_{6} \mathrm{~N}_{4}+\mathrm{H}$.

Allantoinc or allantoic acid occurs in the allantoic fluid of the cow ; it is formed when uric acid is boiled in water with peroxide of lead. It is $\mathrm{C}_{4} \mathrm{H}_{3} \mathrm{~N}_{2} \mathrm{O}_{3}$ or $2 \mathrm{Cy}+3 \mathrm{HO}$. 
from hydrocyanic acid and the elements of water, by various chemical transformations.

The whole process of nutrition may be understood by the consideration of one of these transformations.

Hydrocyanic acid and water, for example, when brought into contact with muriatic acid, are decomposed into formic acid and ammonia; both of these products of decomposition contain the elements of hydrocyanic acid and water, although in another form, and arranged in a different order. The change results from the strong disposition or struggle of muriatic acid to undergo saturation, in consequence of which the hydrocyanic acid and water suffer mutual decomposition. The nitrogen of the hydrocyanic acid and the hydrogen of the water unite together and form a base, ammonia, with which the acid unites; the chemical characters of the acid being at the same time lost, because its desire for saturation is satisfied by its uniting with ammonia. Ammonia itself was not previously present, but only its elements, and the power to form it. The simultaneous decomposition of hydrocyanic acid and water in this instance does not take place in consequence of the chemical affinity of muriatic acid for ammonia, since hydrocyanic acid and water contain no ammonia. An affinity of one body for a second which is totally without the sphere of its attractions, or which, as far as it is concerned, does not exist, is quite inconceivable. The ammonia in this case is formed only on account of the existing attractive desire of the acid for saturation. Hence we may perceive how much these modes of decomposition, to which the name of transformations or metamorphoses has been especially applied, differ from the ordinary chemical decompositions.

In consequence of the formation of ammonia, the other elements of hydrocyanic acid, namely, carbon and hydrogen, unite with the oxygen of the decomposed water, and form formic acid, the elements of this substance with the power of combination being 
present. Formic acid here represents the excrementitious matters; ammonia, the new substance, assimilated by an organ of a plant or animal.

Each organ extracts from the food presented to it what it requires for its own sustenance; while the remaining elements, which are not assimilated, combine together and are separated as excrement. The excrementitious matters of one organ come in contact with another during their passage through the organism, and in consequence suffer new transformations; the useless matters rejected by one organ containing the elements for the nutrition of a second and a third organ: but at last, being capable of no further transformations, they are separated from the system by the organs destined for that purpose. Each part of an organized being is fitted for its peculiar functions. A cubic inch of sulphuretted hydrogen introduced into the lungs would cause instant death, but it is formed, under a variety of circumstances, in the intestinal canal without any injurious effect.*

In consequence of such transformations as we have described, excrements are formed of various composition; some of these contain carbon in excess, others nitrogen, and others again hydrogen and oxygen. The kidneys, liver, and lungs, are organs of excretion; the first separate from the body all those substances in which a large proportion of nitrogen is contained; the second, those with an excess of carbon; and the third, such as are composed principally of oxygen and hydrogen. Alcohol, also, and the volatile oils which are incapable of being assimilated, are exhaled through the lungs, and not through the skin.

Respiration must be regarded as a slow process of combustion or constant decomposition. If it be subject to the laws which regulate the processes

* The danger of breathing carbonic acid gas is well known, but large quantities can be taken into the stomach with impunity and even benefit. 
of decomposition generally, the oxygen of the inspired air cannot combine directly with the carbon of compounds of that element contained in the blood; the hydrogen only can combine with the oxygen of the air, or undergo a higher degree of oxidation. Oxygen is absorbed without uniting with carbon; and carbonic acid is disengaged, the carbon and oxygen of which must be derived from matters previously existing in the blood.*

All superabundant nitrogen is eliminated from the body, as a liquid excrement, through the urinary passages; all solid substances, incapable of further transformation, pass out by the intestinal canal, and all gaseous matter by the lungs.

We should not permit ourselves to be withheld by the idea of a vital principle, from considering in a chemical point of view the process of the transformation of the food, and its assimilation by the various organs. This is the more necessary, as the views, hitherto held, have produced no results, and are quite incapable of useful application.

Is it truly vitality, which generates sugar in the germ for the nutrition of young plants, or which gives to the stomach the power to dissolve, and to prepare for assimilation, all the matter introduced into it? A decoction of malt possesses as little power to reproduce itself, as the stomach of a dead calf; both are, unquestionably, destitute of life.

* The examination of the air expired by consumptive persons, as well as of their blood, would doubtless throw much light on the nature of phthisis pulmonulis. Considered in a chemical point of view, the decomposition of the blood, as it takes place in the lungs, is a true process of putrefaction. (See Part II.) The lungs are also the seat of the transformation of the various substances contained in the blood. It certainly well merits consideration, that the most approved remedies for counteracting or stopping the progress of this frightful malady are precisely those which are found most efficacious in retarding putrefaction. Thus, it is well known, that much relief is afforded by a residence in works in which empyreumatic oils are manufactured by: dry distillation, such as manufactories for the preparation of gas or salammoniac. For the same reason, the respiration of wood vinegar(pyroligneous acid), of chlorine, and certain of the acids, has been: recognised as a means of alleviating the disease. $-L$. 
But when amylin or starch is introduced into a decoction of malt, it changes, first into a gummy-like matter, and lastly into sugar. Hard-boiled albumen and muscular fibre can be dissolved in a decoction of a calf's stomach, to which a few drops of muriatic acid have been added, precisely as in the stomach itself.* (Schwann, Schulz.)

The power, therefore, to effect transformations, does not belong to the vital principle: each transformation is owing to a disturbance in the attraction of the elements of a compound, and is consequently a purely chemical process. There is no doubt that this process takes place in another form from that of the ordinary decomposition of salts, oxides, or sulphurets. But is it the fault of chemistry that physiology has hitherto taken no notice of this new form of chemical action?

Physicians are accustomed to administer whole ounces of borax to patients suffering under urinary calculi, when it is known that the bases of all alkaline salts formed by organic acids are carried through the urinary passages in the form of alkaline carbonates, capable of dissolving calculi (Wöhler). Is this rational? The medical reports state, that upon the Rhine, where so much cream of tartar is consumed in wine, the only cases of calculous disorders are those which are imported from other districts. We know that the uric acid calculus is transformed into the mulberry calculus (which contains oxalic acid), when patients suffering under the former exchange the town for the country, where less animal and more vegetable food is used. Are all these circumstances incapable of explanation?

The volatile oil of the roots of valerian may be obtained from the oil generated during the fermentation of potatoes (Dumas), and the oil of the Spircea ulmaria from the crystalline matter of the

* This remarkable action has been completely confirmed in this laboratory (Giessen), by Dr. Yogel, a highly distinguished young physiologist. $-L$. 
bark of the willow (Piria). We are able to form in our laboratories formic acid, oxalic acid, urea, and the crystalline substances existing in the liquid of the allantois of the cow, all products, it is said, of the vital principle. We see, therefore, that this mysterious principle has many relations in common with chemical forces, and that the latter can indeed replace it. What these relations are, it remains for physiologists to investigate. Truly it would be extraordinary if this vital principle, which uses everything for its own purposes, had allotted no share to chemical forces, which stand so freely at its disposal. We shall obtain that which is obtainable in a rational inquiry into nature, if we separate the actions belonging to chemical powers from those which are subordinate to other influences. But the expression "vital principle" must in the mean time be considered as of equal value with the terms specific or dynamic in medicine: everything is specific which we cannot explain, and dynamic is the explanation of all which we do not understand; the terms having been invented merely for the purpose of concealing ignorance by the application of learned epithets.

Transformations of existing compounds are constantly taking place during the whole life of a plant, in consequence of which, and as the results of these transformations, there are produced gaseous matters which are excreted by the leaves and blossoms, solid excrements deposited in the bark, and fluid soluble substances which are eliminated by the roots. Such secretions are most abundant immediately before the formation and during the continuance of the blossoms; they diminish after the development of the fruit. Substances containing a large proportion of carbon are excreted by the roots and absorbed by the soil. Through the expulsion of these matters unfitted for nutrition, the soil receives again with usury the carbon which it had at 
first yielded to the young plants as food, in the form of carbonic acid.

The soluble matter thus acquired by the soil is still capable of decay and putrefaction, and by undergoing these processes furnishes renewed sources of nutrition to another generation of plants; it becomes humus. The cultivated soil is thus placed in a situation exactly analogous to that of forests and meadows; for the leaves of trees which fall in the forest in autumn, and the old roots of grass in the meadow, are likewise converted into humus by the same influence: a soil receives more carbon in this form than its decaying humus had lost as carbonic acid.

Plants do not exhaust the carbon of a soil in the normal condition of their growth; on the contrary, they add to its quantity. But if it is true that plants give back more carbon to a soil than they take from it, it is evident that their growth must depend upon the reception of nourishment from the atmosphere in the form of carbonic acid. The influence of humus upon vegetation is explained by the foregoing facts in the most clear and satisfactory manner.

Humus does not nourish plants by being taken up and assimilated in its unaltered state, but by presenting a slow and lasting source of carbonic acid, which is absorbed by the roots, and is the principal nutriment of young plants at a time when, being destitute of leaves, they are unable to extract food from the atmosphere.

In former periods of the earth's history; its surface was covered with plants, the remains of which are still found in the coal formations. These plants, - the gigantic monocotyledons, ferns, palms, and reeds, - belong to a class to which nature has given the power, by means of an immense extension of their leaves, to dispense with nourishment from the soil. They resemble in this respect the plants which we raise from bulbs and tubers, and which live while young upon the substances contained in their seed, 
and require no food from the soil when their exterior organs of nutrition are formed. This class of plants is even at present ranked amongst those which do not exhaust the soil.

'The necessity of the existence of plants such as these at the commencement of vegetation, must now be apparent. Humus is a product of the decay of vegetable matter, and therefore could not have existed to supply the first plants with the food necessary for the development of the more delicate kinds. Hence the plants capable of flourishing under such circumstances could only be those which receive their nourishment from the air alone. By their decay, however, the soil in which they grew became supplied with vegetable matter, and the progress of vegetation must have furnished to the earth materials adapted for the development of those plants, which depend upon the nutriment contained in the soil, until those organs are formed which are destined for the assumption of nourishment from the atmosphere.

The plants of every former period are distinguished from those of the present by the inconsiderable development of their roots. Fruit, leaves, seeds, nearly every part of the plants of a former world, except the roots, are found in the brown coal formation. The vascular bundles, and the perishable cellular tissue, of which their roots consisted, have been the first to suffer decomposition. But when we examine oaks and other trees, which in consequence of revolutions of the same kind occurring in later ages have undergone the same changes, we never find their roots absent.

The verdant plants of warm climates are very often such as obtain from the soil only a point of attachment, and are not dependent on it for their growth. How extremely small are the roots of the Cactus, Sedum, and Sempervivum, in proportion to their mass, and to the surface of their leaves! Large forests are often found growing in soils absolutely des- 
titute of carbonaceous matter; and the extensive prairies of the Western Continent show that the carbon necessary for the sustenance of a plant may be entirely extracted from the atmosphere. Again, in the most dry and barren sand, where it is impossible for nourishment to be obtained through the roots, we see the milky-juiced plants attain complete perfection. The moisture necessary for the nutrition of these plants is derived from the atmosphere, and when assimilated is secured from evaporation by the nature of the juice itself. Caoutchouc and wax, which are formed in these plants, surround the water, as in oily emulsions, with an impenetrable envelope by which the fluid is retained, in the same manner as milk is prevented from evaporating by the skin which forms upon it. These plants, therefore, become turgid with their juices.

Particular examples might be cited of plants, which have been brought to maturity, upon a small scale, without the assistance of mould; but fresh proofs of the accuracy of our theory respecting the origin of carbon would be superfluous and useless, and could not render more striking; or more convincing, the arguments already adduced. It must not, however, be left unmentioned, that common wood charcoal, by virtue merely of its ordinary well-known properties, can completely replace vegetable mould or humus. The experiments of Lukas, which are appended to this work, spare me all further remarks upon its efficacy.

Plants thrive in powdered charcoal, and may be brought to blossom and bear fruit if exposed to the influence of the rain and the atmosphere; the charcoal may be previously heated to redness. Charcoal is the most "indifferent" and most unchangeable substance known; it may be kept for centuries without change, and is therefore not subject to decomposition. The only substances which it can yield to plants are some salts, which it contains, amongst which is silicate of potash. It is known, however, 
to possess the power of condensing gases within its pores, and particularly carbonic acid. And it is by virtue of this power that the roots of plants are supplied in charcoal, exactly as in humus, with an atmosphere of carbonic acid and air, which is renewed as quickly as it is abstracted.

In charcoal powder, which had been used for this purpose by Lukas for several years, Buchner found a brown substance soluble in alkalies. This substance was evidently due to the secretions from the roots of the plants which grew in it.

A plant placed in a closed vessel in which the air, and therefore the carbonic acid, cannot be renewed, dies exactly as it would do in the vacuum of an airpump, or in an atmosphere of nitrogen or carbonic acid, even though its roots be fixed in the richest mould.*

Plants do not, however, attain maturity, under ordinary circumstances, in charcoal powder, when they are moistened with pure distilled water instead of rain or river water. Rain water must, therefore, contain within it one of the essentials of vegetable life; and it will be shown, that this is the presence of a compound containing nitrogen, the exclusion of which entirely deprives humus and charcoal of their influence upon vegetation.

* A few years since I had an opportunity of observing a striking instance of the effect of carbonic acid upon vegetation in the volcanic island of St. Michael (Azores). The gas issued from a fissure in the base of a hill of trachyte and tuffa from which a level field of some acres extended. This field, at the time of my visit, was in part covered with Indian corn. The corn at the distance of ten or fifteen yards from the fissure, was nearly full grown, and of the usual height, but the height regularly diminished until within five or six feet of the hill, where it attained but a few inches. This effect was owing to the great specific gravity of the carbonic acid, and its spreading upon the ground, but as the distance increased, and it became more and more mingled with atmospheric air, it had produced less and less effect. $-W$. 


\section{CHAPTER IV.}

\section{ON THE ASSIMILATION OF HYDROGEN.}

THE atmosphere contains the principal food of plants in the form of carbonic acid, in the state, therefore, of an oxide. The solid part of plants (woody fibre) contains carbon and the constituents of water, or the elements of carbonic acid, together. with a certain quantity of hydrogen. It has formerly been mentioned that water consists of the two gases, oxygen and hydrogen. The range of affinity possessed by both these elements is so extensive, that numerous causes occur which effect the decomposition of water. Indeed, there is no compound which plays a more general or more important part in the phenomena of combination and decomposition. We can conceive the wood to arise from a combination of the carbon of the carbonic acid with the elements of water, under the influence of solar light. In this case, 72.35 parts of oxygen, by weight, must be separated as a gas for every 27.65 parts of carbon, which are assimilated by a plant; for this is the composition of carbonic acid in 100 parts. Or, what is much more probable, plants, under the same circumstances, may decompose water, the hydrogen of which is assimilated along with carbonic acid, whilst its oxygen is separated. If the latter change takes place, 8.04 parts of hydrogen must unite with 100 parts of carbonic acid, in order to form woody fibre, and the 72.35 parts by weight of oxygen, which was in combination with the hydrogen of the water, and which exactly corresponds in quantity with the oxygen contained in the carbonic acid, must be separated in a gaseous form.

Each acre of land, which produces $10 \mathrm{cwts}$. of carbon, gives annually to the atmosphere $2865 \mathrm{lbs}$. of free oxygen gas. The specific weight of oxygen is 
expressed by the number $1 \cdot 1026$; hence 1 cubic metre of oxygen weighs $3.157 \mathrm{lbs}$., and $2865 \mathrm{lbs}$. of oxygen correspond to 908 cubic metres, or 32,007 cubic feet.

An acre of meadow, wood, or cultivated land in general replaces, therefore, in the atmosphere as much oxygen as is exhausted by $10 \mathrm{cwts}$. of carbon, either in its ordinary combustion in the air or in the respiratory process of animals.

It has been mentioned at a former page that pure woody fibre contains carbon and the component parts of water, but that ordinary wood contains more hydrogen than corresponds to this proportion. This excess is owing to the presence of the green principle of the leaf, wax, resin, and other bodies rich in hydrogen. Water must be decomposed, in order to furnish the excess of this element, and consequently one equivalent of oxygen must be given back to the atmosphere for every equivalent of hydrogen appropriated by a plant to the production of those substances. The quantity of oxygen thus set at liberty cannot be insignificant, for the atmosphere must receive 547 cubic feet of oxygen for every pound of hydrogen assimilated.

It has already been stated, that a plant, in the formation of woody fibre, must always yield to the atmosphere the same proportional quantity of oxygen; that the volume of this gas set free would be the same whether it were due to the decomposition of carbonic acid or of water. A little consideration will show that this must be the case. It has repeatedly been stated, that woody fibre contains carbon in combination with oxygen and hydrogen in the same proportion in which they exist in water. Water contains 1 equivalent of each element, whilst carbonic acid consists of 1 equivalent of carbon, united to 2 equivalents of oxygen. In the formation of woody fibre, 2 equivalents of oxygen must therefore be liberated. The woody fibre can only be formed in one of two ways: either the carbon of carbonic acid 
unites directly with water, or the hydrogen of water combines with the oxygen of the carbonic acid. In the former of these cases, the two equivalents of oxygen in the carbonic acid must be liberated; in the latter, two atoms of water must be decomposed, the hydrogen of which unites with the oxygen of the carbonic acid, whilst the oxygen of the water, thus set free, is disengaged in the state of a gas. It was considered most probable that the latter was the case.

From their generating caoutchouc, wax, fats, and volatile oils containing hydrogen in large quantity, and no oxygen, we may be certain that plants possess the property of decomposing water, because from no other body could they obtain the hydrogen of those matters. It has also been proved by the observations of Humboldt on the fungi, that water may be decomposed without the assimilation of hydrogen. Water is - a remarkable combination of two elements, which have the power to separate themselves from one another, in innumerable processes, in a manner imperceptible to our senses ; while carbonic acid, on the contrary, is only decomposable by violent chemical action.

Most vegetable structures contain hydrogen in the form of water, which can be separated as such, and replaced by other bodies; but the hydrogen which is essential to their constitution cannot possibly exist in the state of water.

All the hydrogen necessary for the formation of an organic compound is supplied to a plant by the decomposition of water. The process of assimilation, in its most simple form, consists in the extraction of hydrogen from water, and carbon from carbonic acid, in consequence of which, either all the oxygen of the water and carbonic acid is separated, as in the formation of caoutchouc, the volatile oils which contain no oxygen, and other similar substances, or only a part of it is exhaled.

The known composition of the organic compounds 
most generally present in vegetables, enables us to state in definite proportions the quantity of oxygen separated during their formation.

36 eq. carbonic acid and 22 eq. hydrogen derived
from 22 eq. water

with the separation of 72 eq. oxygen.

36 eq. carbonic acid and 36 eq. hydrogen derived
from 36 eq. water

with the separation of 72 eq. oxygen.

36 eq. carbonic acid and 30 eq. hydrogen derived
from $30 \mathrm{eq}$. water

with the separation of 72 eq. oxygen.

36 eq. carbonic acid and 16 eq. hydrogen derived
from 16 eq. water

with the separation of 64 eq. oxygen.

36 eq. carbonic acid and 18 eq. hydrogen derived

from 18 eq. water

with the separation of 45 eq. oxygen.

36 eq. carbonic acid and 18 eq. hydrogen derived $\}=$ Malic Acid,
from 18 eq. water

with the separation of 54 eq. oxygen.

$\left.\begin{array}{l}36 \text { eq. carbonic acid and } 24 \text { eq. hydrogen derived } \\ \text { from } 24 \text { eq. water }\end{array}\right\}=$ Oil of Turpentine, with the separation of 84 eq. oxygen.

It will readily be perceived, that the formation of the acids is accompanied with the smallest separation of oxygen; that the amount of oxygen set free increases with the production of the sonamed neutral substances, and reaches its maximum in the formation of the oils. Fruits remain acid in cold summers; while the most numerous trees under the tropics are those which produce oils, caoutchouc, and other substances containing very little oxygen. The action of sunshine and influence of heat upon the ripening of fruit is thus, in a certain measure, represented by the numbers above cited.

The green resinous principle of the leaf diminishes in quantity, while oxygen is absorbed, when fruits are ripened in the dark; red and yellow coloring matters are formed; tartaric, citric, and tannic acids disappear, and are replaced by sugar, amylin, or gum. 6 eq. Tartaric Acid, by absorbing 6 eq. oxygen from the air, form Grape Sugar, with the separation of 12 eq. carbonic acid. 1 eq. Tannic Acid, by absorbing 8 eq. oxygen from the air, and 4 eq. 
water, form 1 eq. of Amylin, or starch, with separation of 6 eq. carbonic acid.

We can explain, in a similar manner, the formation of all the component substances of plants which contain no nitrogen, whether they are produced from carbonic acid and water, with separation of oxygen, or by the conversion of one substance into the other, by the assimilation of oxygen and separation of carbonic acid. We do not know in what form the production of these constituents takes place; in this respect, the representation of their formation which we have given must not be received in an absolute sense, it being intended only to render the nature of the process more capable of apprehension; but it must not be forgotten, that if the conversion of tartaric acid into sugar, in grapes, be considered as a fact, it must take place under all circumstances in the same proportions.

The vital process in plants is, with reference to the point we have been considering, the very reverse of the chemical processes engaged in the formation of salts. Carbonic acid, zinc, and water, when brought into contact, act upon one another, and hydrogen is separated, while a white pulverulent compound is formed, which contains carbonic acid, zinc, and the oxygen of the water. A living plant represents the zinc in this process: but the process of assimilation gives rise to compounds, which contain the elements of carbonic acid and the hydrogen of water, whilst oxygen is separated.

Decay has been described above as the great operation of nature, by which that oxygen, which was assimilated by plants during life, is again returned to the atmosphere. During the progress of growth, plants appropriate carbon in the form of carbonic acid, and hydrogen from the decomposition of water, the oxygen of which is set free, together with a part of all of that contained in the carbonic acid. In the process of putrefaction, a quantity of water, exactly corresponding to that of the hydro- 
gen, is again formed by extraction of oxygen from the air; while all the oxygen of the organic matter is returned to the atmosphere in the form of carbonic acid: Vegetable matters can emit carbonic acid, during their decay, only in proportion to the quantity of oxygen which they contain; acids, therefore, yield more carbonic acid than neutral compounds; while fatty acids, resin, and wax, do not putrefy; they remain in the soil without any apparent change.

The numerous springs which emit carbonic acid in the neighborhood of extinct volcanoes, must be regarded as another means of compensating for the carbonic acid absorbed and retained by plants during life, and consequently as a source by which oxygen is supplied to the atmosphere. Bischof calculated that the springs of carbonic acid in the Eifel (a volcanic district near Coblenz) send into the air every day more than $99,000 \mathrm{lbs}$. of carbonic acid, corresponding to $71,000 \mathrm{lbs}$. of pure oxygen.

\section{CHAPTER V.}

\section{ON THE ORIGIN AND ASSIMILATION OF NITROGEN.}

WE cannot suppose that a plant could attain maturity, even in the richest vegetable mould, without the presence of matter containing nitrogen; since we know that nitrogen exists in every part of the vegetable structure. The first and most important question to be solved, therefore, is: How and in what form does nature furnish nitrogen to vegetable albumen, and gluten, to fruits and seeds?

This question is susceptible of a very simple solution.

Plants, as we know, grow perfectly well in pure charcoal, if supplied at the same time with rainwater. Rain-water can contain nitrogen only in two forms, either as dissolved atmospheric air, or as. 
ammonia, which consists of this element and hydrogen. Now, the nitrogen of the air cannot be made to enter into combination with any element except oxygen, even by the employment of the most powerful chemical means. We have not the slightest reason for believing that the nitrogen of the atmosphere takes part in the processes of assimilation of plants and animals; on the contrary, we know that many plants emit the nitrogen which is absorbed by their roots, either in the gaseous form, or in solution in water. But there are on the other hand numerous facts, showing, that the formation in plants of substances containing nitrogen, such as gluten, takes place in proportion to the quantity of this element which is conveyed to their roots in the state of ammonia, ${ }^{*}$ derived from the putrefaction of animal matter.

Ammonia, too, is capable of undergoing such a multitude of transformations, when in contact with other bodies, that in this respect it is not inferior to water, which possesses the same property in an eminent degree. It possesses properties which we do not find in any other compound of nitrogen: when pure, it is extremely soluble in water; it forms soluble compounds with all the acids; and when in contact with certain other substances, it completely resigns its character as an alkali, and is capable of assuming the most various and opposite forms. Formate of ammonia $f$ changes, under the influence of a high temperature, into hydrocyanic acid and water, without the separation of any of its elements.

* Ammonia is a compound gas, consisting of one volume of nitrogen and three volumes of hydrogen. It is produced during the decomposition of many animal substances. It is given off when sal-ammoniac and lime are rubbed together. It was formerly called volatile alkali.

+ Formic acid (p. 70.n.) is also obtained from sugar and many other vegetable substances; a pound of sugar yields a quantity capable of saturating five or six ounces of carbonate of lime. A process for obtaining it has been given by Emmet in the American Journal, Vol. XXXII. p. 140. See details in WeBster's Manual of Chemistry, 3d edition, p. 374 .

Its composition is carbon 2 , water 3 . With ammonia and other bases it yields the salts called formates. 
Ammonia forms urea, * with cyanic acid, $\dagger$ and a series of crystalline compounds, with the volatile oils of mustard and bitter almonds. It changes into splendid blue or red coloring matters, when in contact with the bitter constituent of the bark of the apple-tree (phloridzin), with the sweet principle of the Variolaria dealbata (orcin), or with the tasteless matter of the Rocella tinctoria (erythrin). All blue coloring matters which are reddened by acids, and all red coloring substances which are rendered blue by alkalies, contain nitrogen, but not in the form of a base.

These facts are not sufficient to establish the opinion that it is ammonia which affords all vegetables, without exception, the nitrogen which enters into the composition of their constituent substances. Considerations of another kind, however, give to this opinion a degree of certainty which completely excludes all other views of the matter.

Let us picture to ourselves the condition of a well-cultured farm, so large as to be independent of assistance from other quarters. On this extent of land there is a certain quantity of nitrogen contained both in corn and fruit which it produces, and in the men and animals which feed upon them, and also in their excrements. We shall suppose this quantity to be known. The land is cultivated without the importation of any foreign substance containing nitrogen. Now, the products of this farm must be exchanged every year for money, and other necessaries of life - for bodies, therefore, which contain no nitrogen. A certain proportion of nitrogen is exported with corn and cattle; and this exportation takes place every year, without the smallest compensation; yet after a given number of years, the quantity of nitrogen will be found to have increased.

* Urea was discovered in urine, being a constituent of uric acid. It contains the elements of cyanate of ammonia $\left(\mathrm{NH}_{4} \mathrm{O}+\mathrm{C}_{4} \mathrm{NO}\right)$.

+ This acid consists of 1 cyanogen and 1 oxygen. See Webster's Chemistry, p. 398. 
Whence, we may ask, comes this increase of nitrogen? The nitrogen in the excrements cannot reproduce itself, and the earth cannot yield it. Plants, and consequently animals, must, therefore, derive their nitrogen from the atmosphere.

It will in a subsequent part of this work be shown, that the last products of the decay and putrefaction of animal bodies present themselves in two different forms. They are in the form of a combination of hydrogen and nitrogen, - ammonia, - in the temperate and cold climates, and in that of a compound containing oxygen, - nitric acid, - in the tropics and hot climates. The formation of the latter is preceded by the production of the first. Ammonia is the last product of the putrefaction of animal bodies ; nitric acid is the product of the transformation of ammonia. A generation of a thousand million men is renewed every thirty years : thousands of millions of animals cease to live, and are reproduced, in a much shorter period. Where is the nitrogen which they contained during life? There is no question which can be answered with more positive certainty. All animal bodies during their decay yield the nitrogen which they contain to the atmosphere, in the form of ammonia. Even in the bodies buried sixty feet under ground in the churchyard of the Eglise des Innocens, at Paris, all the nitrogen contained in the adipocire was in the state of ammonia.* Ammonia is the simplest of all compounds of nitrogen; and hydrogen is the element for which nitrogen possesses the most powerful affinity.

The nitrogen of putrefied animals is contained in the atmosphere as ammonia, in the form of a gas

* In 1786-7, when this churchyard was cleared out, it was discovered that many of the bodies had been converted into a soapy. white substance. Fourcroy attempted to prove that the fatty body was an ammoniacal soap, containing phosphate of lime, that the fat was similar to spermaceti and to wax, hence he called it adipocire. Its melting point was $126.5^{\circ} \mathrm{F}$.

For notice of the analysis and opinions of other chemists, see URE's Dictionary of Arts and Manufactures, p. 14. 
which is capable of entering into combination with carbonic acid and of forming a volatile salt. Ammonia in its gaseous form, as well as all its volatile compounds, is of extreme solubility in water.* Ammonia, therefore, cannot remain long in the atmosphere, as every shower of rain must condense it, and convey it to the surface of the earth. Hence also, rain-water must at all times contain ammonia, though not always in equal quantity. It must be greater in summer than in spring or in winter, because the intervals of time between the showers are in summer greater; and when several wet days occur, the rain of the first must contain more of it than that of the second. The rain of a thunder-storm, after a longprotracted drought, ought for this reason to contain the greatest quantity which is conveyed to the earth at one time.

But we have formerly stated, that all the analyses of atmospheric air hitherto made have failed to demonstrate the presence of ammonia, although, according to our view, it can never be absent. Is it possible that it could have escaped our most delicate and most exact apparatus? The quantity of nitrogen contained in a cubic foot of air is certainly extremely small, but, notwithstanding this, the sum of the quantities of nitrogen from thousands and millions of dead animals is more than sufficient to supply all those living at one time with this element.

From the tension of aqueous vapor at $15^{\circ} \mathrm{C}$. (59 $\mathrm{F}.)=6,98$ lines (Paris measure), and from its known specific gravity at $0^{\circ}$ C. $\left(32^{\circ} \mathrm{F}\right.$.), it follows that when the temperature of the air is $59^{\circ} \mathrm{F}$. and the height of the barometer $28^{\prime \prime}, 1$ cubic metre or $35 \cdot 3$ cubic feet of aqueous vapor are contained in 487 cubic metres, or 17,198 cubic feet of air ; $35 \cdot 3$ cubic feet of aqueous vapor weigh about $1.65 \mathrm{lb}$. Consequently, if we suppose that the air saturated with moisture at $59^{\circ} \mathrm{F}$. allows all the water which it con-

* According to Dr. Thomson, water absorbs 780 times its bulk of ammonia. 
tains in the gaseous form to fall as rain, then $1 \cdot 1$ pound of rain water must be obtained from every 11,477 cubic feet of air. The whole quantity of ammonia contained in the same number of cubic feet will also be returned to the earth in this one pound of rain-water. But if the 11,477 cubic feet of air contain a single grain of ammonia, then ten cubic inches, - the quantity usually employed in an analysis, - must contain only 0.000000050 of a grain. This extremely small proportion is absolutely inappreciable by the most delicate and best eudiometer; * it might be classed among the errors of observation, even were its quantity ten thousand times greater. But the detection of ammonia must be much more easy when a pound of rain-water is examined, for this contains all the gas that was diffused through 11,477 cubic feet of air.

If a pound of rain-water contain only $\frac{1}{4}$ th of a grain of ammonia, then a field of 26,910 square feet must receive annually upwards of $88 \mathrm{lbs}$. of ammonia, or $71 \mathrm{lbs}$. of nitrogen; for by the observations of Schubler, which were formerly alluded to, about 770,000 lbs. of rain fall over this surface in four months, and consequently the annual fall must be $2,310,000 \mathrm{lbs}$. This is much more nitrogen than is contained in the form of vegetable albumen and gluten, in $2920 \mathrm{lbs}$. of wood, $3085 \mathrm{lbs}$. of hay, or 200 cwt. of beet-root, which are the yearly produce of such a field; but it is less than the straw, roots, and grain of corn, which might grow on the same surface, would contain. $f$

* A eudiometer is an instrument used in the analyses of the atmo. sphere. It means a measure of purity. It is also used in the analysis of mixtures of gases. Several varieties are described in WEBsTER's Manual, p. 137.

+ The advocates of the importance of humus as a nourishment for plants, being driven from their position by the facts brought forward in the preceding chapters, have found in the ammonia of the atmosphere an explanation of the manner in which humus acquires its solubility, and therefore its capability of being assimilated by plants. Now, it is very true that humic acid is soluble in ammonia; but the humic acid of chemists is not contained in soils. Were it so, on treating mould with water we should obtain a dark-colored solution of humate of ammonia. But we obtain a solution which is entirely devoid of this acid. It can- 
Experiments made in this laboratory (Giessen) with the greatest care and exactness have placed the presence of ammonia in rain-water beyond all doubt. It has hitherto escaped observation, because no person thought of searching for it.* All the rain-water employed in this inquiry was collected 600 paces southwest of Giessen, whilst the wind was blowing in the direction of the town. When several hundred pounds of it were distilled in a copper still, and the first two or three pounds evaporated with the addition of a little muriatic acid, a very distinct crystallization of sal-ammoniac was obtained: the crystals had always a brown or yellow color.

Ammonia may likewise be always detected in snowwater. Crystals of sal-ammoniac were obtained by evaporating in a vessel with muriatic acid several pounds of snow, which were gathered from the surface of the ground in March when the snow had a depth of 10 inches. Ammonia was set free from these crystals by the addition of hydrate of lime. The inferior layers of snow which rested upon the ground contained a quantity decidedly greater than those which formed the surface. $\dagger$

It is worthy of observation, that the ammonia contained in rain and snow water possesses an offensive smell of perspiration and animal excrements, - a fact which leaves no doubt respecting its origin.

not be too distinctly kept in mind that humic acid is the product of the decomposition of humus, by means of caustic alkalies. Again, if the colored solutions of humates of ammonia, lime, or magnesia, be poured upon good mould or decayed oak-wood (which is nearly pure humus), and allowed to filter, the solutions are observed to pass through quite colorless ; they are decolorized just as if they had been filtered through charcoal. Here, then, humus possesses the property of extracting humic acid from water; or, in other words, soils have the power of rendering humic acid insoluble, or unfit for assimilation. - ED.

* It has been discovered by Mr. Hayes in rain-water in Vermont, - and in hailstones by M. Girardin, see London and Edinburgh Philosophical Magazine, 1839, Vol. XV. p. 252. See note in APPENDix.

$\uparrow$ Johnston detected it in snow which fell at Durham, G. B., by adding two drops of sulphuric acid to four pints of snow-water, evaporating to dryness, and mixing the dry mass with quicklime or caustic potash. The residual mass contained a brown organic matter, mixed with the sulphate of ammonia. 
Hünefield has proved that all the springs in Greifswalde, Wick, Eldena, and Kostenhagen, contain carbonate and nitrate of ammonia. Ammoniacal salts have been discovered in many mineral springs in Kissingen and other places. The ammonia of these salts can only arise from the atmosphere.

Any one may satisfy himself of the presence of ammonia in rain by simply adding a little sulphuric or muriatic acid to a quantity of rain-wafer, and evaporating this nearly to dryness in a clean porcelain basin. The ammonia remains in the residue, in combination with the acid employed; and may be detected either by the addition of a little chloride of platinum, or more simply by a little powdered lime, which separates the ammonia, and thus renders its peculiar pungent smell sensible.* The sensation which is perceived upon moistening the hand with rain-water, so different from that produced by pure distilled water, and to which the term softness is vulgarly applied, is also due to the carbonate of ammonia contained in the former. $\dagger$

The ammonia which is removed from the atmosphere by rain and other causes, is as constantly replaced by the putrefaction of animal and vegetable matters. A certain portion of that which falls with the rain evaporates again with the water, but another portion is, we suppose, taken up by the roots of plants, and, entering into new combinations in the different organs of assimilation, produces albumen, gluten, quinine, morphia, cyanogen, and a number of other compounds containing nitrogen. The chemical characters of ammonia render it capable of

* Since the appearance of the first edition, this experiment has been repeated by many in France, Germany, America, and England, and the existence of ammonia in the atmosphere has been completely confirmed. The assertion, that this ammonia possesses the "offensive smell of perspiration and animal excrements," has been ridiculed by many as fanciful, - by none, however, who have made the experiment. The experiment is so exceedingly easy to perform, that any one may convince himself of the accuracy of the statement. - ED.

$\dagger$ A small quantity of ammonia voater, added to what is commonly called hard water, will give it the softness of rain or snow water. 
entering into such combinations, and of undergoing numerous transformations. We have now only to consider whether it really is taken up in the form of ammonia by the roots of plants, and in that form applied by their organs to the production of the azotized matters contained in them. This question is susceptible of easy solution by well-known facts.

In the year 1834, I was engaged with Dr. Wilbrand, Professor of Botany in the University of Giessen, in an investigation respecting the quantity of sugar contained in different varieties of mapletrees, which grew upon soils which were not manured. We obtained crystallized sugars from all, by simply evaporating their juices, without the addition of any foreign substance ; and we unexpectedly made the observation, that a great quantity of ammonia was emitted from this juice when mixed with lime, and also from the sugar itself during its refinement. The vessels which hung upon the trees in order to collect the juice were watched with greater attention, on account of the suspicion that some evil-disposed persons had introduced urine into them, but still a large quantity of ammonia was again found in the form of neutral salts. The juice had no color, and had no reaction on that of vegetables. Similar observations were made upon the juice of the birch tree; the specimens subjected to experiment were taken from a wood several miles distant from any house, and yet the clarified juice, evaporated with lime, emitted a strong odor of ammonia.

In the manufactories of beet-root sugar, many thousand cubic feet of juice are daily purified with lime, in order to free it from vegetable albumen and gluten, and it is afterwards evaporated for crystallization. Every person who has entered such a manufactory must have been astonished at the great quantity of ammonia which is volatilized along with the steam. This ammonia must be contained in the form of an ammoniacal salt, because the neutral 
juice possesses the same characters as the solution of such a salt in water; it acquires, namely, an acid reaction during evaporation, in consequence of the neutral salt being converted by loss of ammonia into an acid salt. The free acid which is thus formed is a source of loss to the manufacturers of sugar from beet-root, by changing a part of the sugar into uncrystallizable grape sugar and syrup.

The products of the distillation of flowers, herbs, and roots, with water, and all extracts of plants made for medicinal purposes, contain amroonia. The unripe, transparent, and gelatinous pulp of the almond and peach emit much ammonia when treated with alkalies. (Robiquet.) The juice of the fresh tobacco leaf contains ammoniacal salts. The water which exudes from a cut vine, when evaporated with a few drops of muriatic acid, also yields a gummy, deliquescent mass, which evolves much ammonia on the addition of lime. Ammonia exists in every part of plants, in the roots (as in beet-root), in the stem (of the maple-tree), and in all blossoms and fruit in an unripe condition.

The juices of the maple and birch contain both sugar and ammonia, and therefore afford all the conditions necessary for the formation of the azotized components of the branches, blossoms, and leaves, as well as of those which contain no azote or nitrogen. In proportion as the development of those parts advances, the ammonia diminishes in quantity, and when they are fully formed, the tree yields no more juice.

The employment of animal manure in the cultivation of grain, and the vegetables which serve for fodder to cattle, is the most convincing proof that the nitrogen of vegetables is derived from ammonia. The quantity of gluten in wheat, rye, and barley, is very different; these kinds of grain also, even when ripe, contain this compound of nitrogen in very different proportions. Proust found French wheat to contain 12.5 per cent. of gluten; Vogel found that 
the Bavarian contained 24 per cent.; Davy obtained 19 per cent. from winter, and 24 from summer wheat; from Sicilian 21, and from Barbary wheat 19 per cent. The meal of Alsace wheat contains, according to Boussingault, $17 \cdot 3$ per cent. of gluten; that of wheat grown in the "Jardin des Plantes" 26.7 , and that of winter wheat 3.33 per cent. Such great differences must be owing to some cause, and this we find in the different methods of cultivation. An increase of animal manure gives rise not only to an increase in the number of seeds, but also to a most remarkable difference in the proportion of the substances containing nitrogen, such as the gluten which they contain.

Animal manure, in as far as regards the assimilation of nitrogen, acts only by the formation of ammonia. One hundred parts of wheat grown on a soil manured with cow-dung (a manure containing the smallest quantity of nitrogen), afforded only 11.95 parts of gluten, and 64.34 parts of amylin, or starch; whilst the same quantity, grown on a soil manured with human urine, yielded the maximum of gluten, namely $35 \cdot 1$ per cent. Putrefied urine contains nitrogen in the forms of carbonate, phosphate, and lactate of ammonia, and in no other form than that of ammoniacal salts.

"Putrid urine is employed in Flanders as a manure with the best results. During the putrefaction of urine, ammoniacal salts are formed in large quantity, it may be said exclusively; for under the influence of heat and moisture, urea, the most prominent ingredient of the urine, is converted into carbonate of ammonia. The barren soil on the coast of Peru is rendered fertile by means of a manure called Guano, which is collected from several islands in the South Sea**. It is sufficient to add a small quantity of guano to a soil, which consists only of

* The guario, which forms a stratum several feet in thickness upon the surface of these 'islands, consists of the putrid excrements of in- 
sand and clay, in order to procure the richest crop of maize. The soil itself does not contain the smallest particle of organic matter, and the manure employed is formed only of urate, phosphate, oxalate, and carbonate of ammonia, together with a few earthy salts.*"

Ammonia, therefore, must have yielded the nitrogen to these plants. Gluten is obtained not only from corn, but also from grapes and other plants; but that extracted from the grapes is called vegetable albumen, although it is identical in composition and properties with the ordinary gluten.

It is ammonia which yields nitrogen to the vegetable albumen, the principal constituent of plants; and it must be ammonia which forms the red and blue

numerable sea fowl that remain on them during the breeding season. (See the Chapter on Manures.)

According to Fourcroy and Vauquelin it contains a fourth part of its weight of uric acid, with ammonia and potash.

The London and Edinburgh Philosophical Magazine, for July, 1841, contains a new analysis of the guano, made by $M$. Voelckel in the laboratory of Professor Wöhler, and confirms what Klaproth found, viz., that it contains, besides unchanged uric acid, a considerable quantity of two of its usual products of decomposition, viz. oxalic acid and animonia. 100 parts of moist guano, contain,

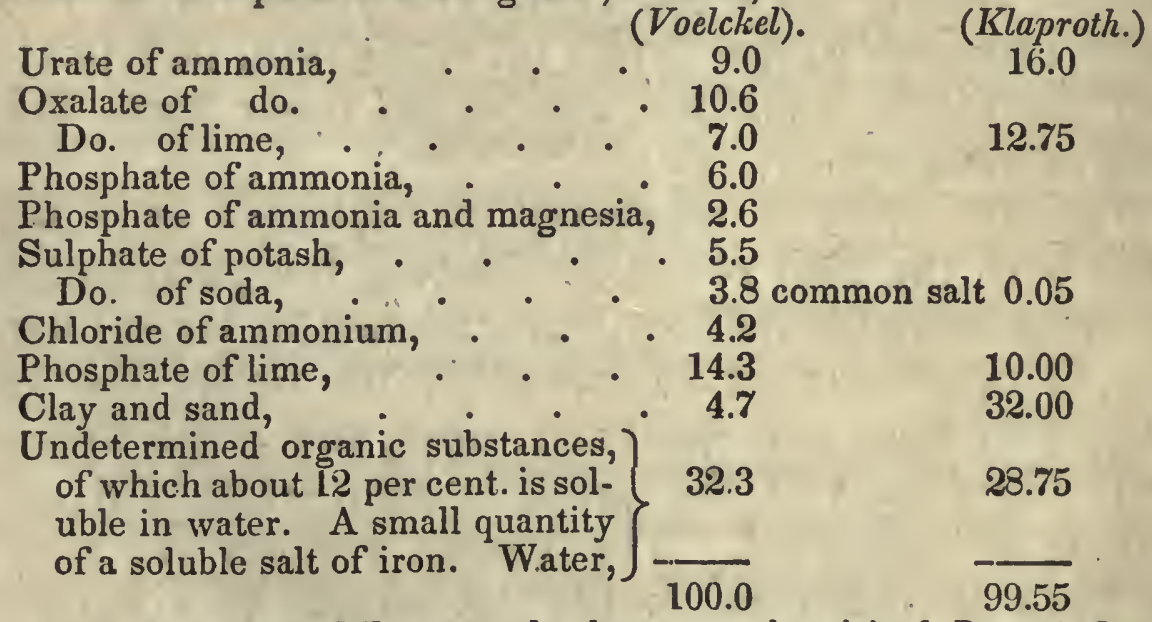

Mr. J. H. Blake of Boston, who has recently visited Peru, informs me, that near Pabellon de Pica there is a high hill, the base of which, consisting chiefly of guano, is washed by the sea. From this bed, which is nearly a mile in length, and from 800 to 900 feet high, guano might be obtained at a cost, which would probably not exceed a cent and a half per pound, delivered in the United States. (See also Appendix.)

* Boussingault. Ann. de Ch. et de Phys. lxv. p. 319. 
coloring matters of flowers. Nitrogen is not presented to wild plants in any other form capable of assimilation. Ammonia, by its transformation, furnishes nitric acid to the tobacco plant, sun-flower, Chenopodium, and Borago officinalis, when they grow in a soil completely free from nitre. Nitrates are necessary constituents of these plants, which thrive only when ammonia is present in large quantity, and when they are also subject to the influence of the direct rays of the sun, an influence necessary to effect the disengagement within their stem and leaves of the oxygen, which shall unite with the ammonia to form nitric acid.

The urine of men and of carnivorous animals contains a large quantity of nitrogen, partly in the form of phosphates, partly as urea. Urea is converted during putrefaction into carbonate of ammonia, that is to say, it takes the form of the very salt which occurs in rain-water. Human urine is the most powerful manure for all vegetables containing nitrogen; that of horses and horned cattle contains less of this element, but infinitely more than the solid excrements of these animals. In addition to urea, the urine of herbivorous animals contains hippuric acid,* which is decomposed during putrefaction into benzoic acid $\dagger$ and ammonia. The latter enters into the composition of the gluten, but the benzoic acid often remains unchanged: for example, in the Anthoxanthum odoratum.

The solid excrements of animals contain comparatively very little nitrogen, but this could not be otherwise. The food taken by animals supports them only in so far as it offers elements for assimilation to the various organs which they may require

* Rouelle announced the discovery of an acid in the urine of the horse, which he called benzoic, but in 1834 Liebig showed that this wasnot benzoic acid, but one easily convertible into it, and distinguished it

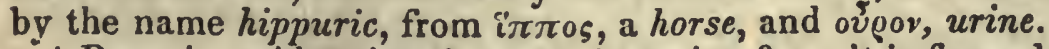

+ Benzoic acid exists in gum benzoin, \&c.; it is formed, according to Liebig, by the oxidation of a supposed base called benzule. Its. composition is carbon 14, hydrogen 5 , oxygen 2. 
for their increase or renewal. Corn, grass, and all plants, without exception, contain azotized substances.* The quantity of food which animals take for their nourishment, diminishes or increases in the same proportion as it contains more or less of the substances containing nitrogen. A horse may be kept alive by feeding it with potatoes, which contain a very small quantity of nitrogen; but life thus supported is a gradual starvation; the animal increases neither in size nor strength, and sinks under every exertion. The quantity of rice which an Indian eats astonishes the European; but the fact that rice contains less nitrogen than any other kind of grain at once explains the circumstance. $\dagger$

Now, as it is evident that the nitrogen of the plants and seeds used by animals as food must be employed in the process of assimilation, it is natural to expect that the excrements of these animals will be deprived of it in proportion to the perfect digestion of the food, and can only contain it when mixed with secretions from the liver and intestines. Under all circumstances, they must contain less nitrogen than the food. When, therefore, a field is manured with animal excrements, a smaller quantity of matter

* The late Professor Gorham obtained from Indian corn a substance to which he gave the name Zeine, according to whose analysis it con. tains no nitrogen; but ammonia has since been obtained from it.

$\dagger$ According to the analysis of Braconnot (Ann. de Chim. et de Phys. t. iv. p. 370 ), this grain is thus constituted.

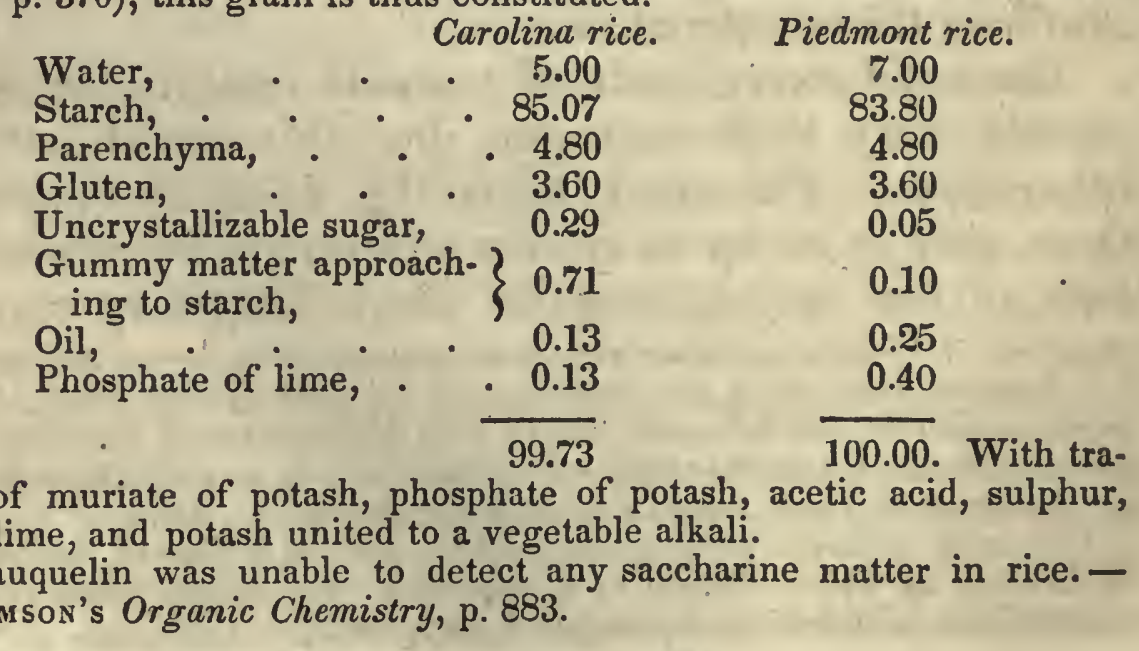


containing nitrogen is added to it than has been taken from it in the form of grass, herbs, or seeds. By means of manure, an addition only is made to the nourishment which the air supplies.

In a scientific point of view, it should be the care of the agriculturist so to employ all the substances containing a large proportion of nitrogen which his farm affords in the form of animal excrements, that they shall serve as nutriment to his own plants. This will not be the case unless those substances are properly distributed upon his land. A heap of manure lying unemployed upon his land would serve him no more than his neighbors. The nitrogen in it would escape as carbonate of ammonia into the atmosphere, and a mere carbonaceous residue of decayed plants would, after some years, be found in its place.

All animal excrements emit carbonic acid and ammonia, as long as nitrogen exists in them. In every stage of their putrefaction an escape of ammonia from them may be induced by moistening them with a potash ley; the ammonia being apparent to the senses by a peculiar smell, and by the dense white vapor which arises when a solid body moistened with an acid is brought near it. This ammonia evolved from manure is imbibed by the soil either in solution in water, or in the gaseous form, and plants thus receive a larger supply of nitrogen than is afforded to them by the atmosphere.

But it is much less the quantity of ammonia, yielded to a soil by animal excrements, than the form in which it is presented by them, that causes their great influence on its fertility. Wild plants obtain more nitrogen from the atmosphere in the form of ammonia than they require for their growth, for the water which evaporates through their leaves and blossoms, emits, afterr some time, a putrid smell, a peculiarity possessed only by such bodies as contain nitrogen. Cultivated plants receive the same quantity of nitrogen from the atmosphere as trees, 
shrubs, and other wild plants; but this is not sufficient for the purposes of agriculture. Agriculture differs essentially from the cultivation of forests, inasmuch as its principal object consists in the production of nitrogen under any form capable of assimilation; whilst the object of forest culture is confined principally to the production of carbon. All the various means of culture are subservient to these two main purposes. A part only of the carbonate of ammonia which is conveyed by rain to the soil is received by plants, because a certain quantity of it is volatilized with the vapor of water; only that portion of it can be assimilated which sinks deeply into the soil, or which is conveyed directly to the leaves by dew, or is absorbed from the air along with the carbonic acid.

Liquid animal excrements, such as the urine with which the solid excrements are impregnated, contain the greatest part of their ammonia in the state of salts, in a form, therefore, in which it has completely lost its volatility; when presented in this condition, not the smallest portion of the ammonia is lost to the plants; it is all dissolved by water, and imbibed by their roots. The evident influence of gypsum upon the growth of grasses - the striking fertility and luxuriance of a meadow upon which it is strewed - depends only upon its fixing in the soil the ammonia of the atmosphere, which would otherwise be volatilized, with the water which evaporates.* The carbonate of ammonia contained in rain-water is decomposed by gypsum, in precisely the same manner as in the manufacture of sal-ammoniac. Soluble sulphate of ammonia and carbonate of lime are formed; and this salt of ammonia possessing no volatility is consequently retained in the soil. All the gypsum gradually disappears, but its action upon

\footnotetext{
* It has long been the practice in some parts of the country to strew the floors of stables with gypsum. This prevents the disagreeable odor arising from the putrefaction of stable manure, by decomposing the ammoniacal salts which are formed.- ED.
} 
the carbonate of ammonia continues as long as a trace of it exists.

The beneficial influence of gypsum and of many other salts has been compared to that of aromatics, which increase the activity of the human stomach and intestines, and give a tone to the whole system. But plants contain no nerves; we know of no substance capable of exciting them to intoxication and madness, or of lulling them to sleep and repose. No substance can possibly cause their leaves to appropriate a greater quantity of carbon from the atmosphere, when the other constituents which the seeds, roots, and leaves require for their growth are wanting.* The favorable action of small quantities of aromatics upon man, when mixed with his food, is undeniable; but aromatics are given to plants without food to be digested, and still they flourish with greater luxuriance.

It is quite evident, therefore, that the common view concerning the influence of certain salts upon the growth of plants evinces only ignorance of its cause.

The action of gypsum or chloride of calcium really consists in their giving a fixed condition to the nitrogen - or ammonia which is brought into the soil, and which is indispensable for the nutrition of plants.

In order to form a conception of the effect of

* In 1831, I suggested to a well known and most successful cultivator (Mr. Haggerston), the application of a weak solution of chlorine gas to the soil in which plants were growing. It appeared to act merely as a stimulant, the plants flourished for a time with great luxuriance, and in some the foliage was remarkable. The leaves of a Pelargonium (well known as the Washington Geranium) attained the diameter of a foot, but the flowers were by no means equal to those of similar plants cultivated in the usual manner; the plants soon perished. Probably a supply of nutriment proportioned to the increased demand was not supplied.

The necessity for this supply is now well known, and Pelargoniums are now grown with great luxuriance and perfection, both of leaves and flowers, by the free use of "manure water," obtained by steeping horsedung in rain-water. The soil, too, best adapted to the plants is chiefly prepared from decayed vegetable matter, derived from decomposed leaves and plants, mixed with that from the sods of fields. 
gypsum, it may be sufficient to remark, that $110 \mathrm{lbs}$. of burned gypsum fixes as much ammonia in the soil as 6887 lbs. of horse's urine* would yield to it, even on the supposition that all the nitrogen of the urea and hippuric acid were absorbed by the plants without the smallest loss, in the form of carbonate of ammonia. If we admit with Boussingault $\dagger$ that the nitrogen in grass amounts to $\frac{1}{100}$ of its weight, then every pound of nitrogen which we add increases the produce of the meadow $110 \mathrm{lbs}$., and this increased produce of $110 \mathrm{lbs}$. is effected by the aid of a little more than $4 \mathrm{lbs}$. of gypsum.

Water is absolutely necessary to effect the decomposition of the gypsum, on account of its difficult solubility, ( 1 part of gypsum requires 400 parts of water for solution, ) and also to assist in the absorption of the sulphate of ammonia by the plants: hence it happens, that the influence of gypsum is not observable on dry fields and meadows. In such it would be advisable to employ a salt of more easy solubility, such as chloride of calcium.

The decomposition of gypsum by carbonate of ammonia does not take place instantaneously; on the contrary, it proceeds very gradually, and this explains why the action of the gypsum lasts for several years.

The advantage of manuring fields with burned clay, and the fertility of ferruginous soils, which have been considered as facts so incomprehensible, may be explained in an equally simple manner. They have been ascribed to the great attraction for water, exerted by dry clay and ferruginous earth; but common dry arable land possesses this property

* The urine of the horse contains, according to Fourcroy and Vauquelin, in 1000 parts,

$$
\begin{aligned}
& \text { Urea } \\
& \text { Salts and water } \\
& \text { Sippurate of soda }
\end{aligned} \cdot \frac{24 \text { parts. }}{979 \text { " }}
$$

† Boussingault, Ann. de Ch. et de Phys., t. lxiii. page 243. 
in as great a degree: and besides, what influence can be ascribed to a hundred pounds of water spread over an acre of land, in a condition in which it cannot be serviceable either by the roots or leaves? The true cause is this:-

The oxides of iron and alumina are distinguished from all other metallic oxides by their power of forming solid compounds with ammonia. The precipitates obtained by the addition of ammonia to salts of alumina or iron are true salts, in which the ammonia is contained as a base. Minerals containing alumina or oxide of iron also possess, in an eminent degree, the remarkable property of attracting ammonia from the atmosphere and of retaining it. Vauquelin, whilst engaged in the trial of a criminal case, discovered that all rust of iron contains. a certain quantity of ammonia. Chevalier afterwards found that ammonia is a constituent of all minerals containing iron; that even hematite, a mineral which is not at all porous, contains one per cent. of it. Bouis showed also, that the peculiar odor observed on moistening minerals containing alumina, is partly owing to their exhaling ammonia. Indeed, gypsum and some varieties of alumina, pipe-clay for example, emit so much ammonia, when moistened with caustic potash, that even after they have been exposed for two days, reddened litmus paper held over them becomes blue. Soils, therefore, which contain oxides of iron, and burned clay, must absorb ammonia, an action which is favored by their porous condition; they further prevent the escape of the ammonia once absorbed, by their chemical properties.' Such soils, in fact, act precisely as a mineral acid would do, if extensively spread over their surface; with this difference, that the acid would penetrate the ground, enter into combination with lime, alumina, and other bases, and thus lose, in a few hours, its property of absorbing ammonia from the atmosphere. The addition of burned clay to soils has also a secondary influence; 
it renders the soil porous, and, therefore, more permeable to air and moisture.

The ammonia absorbed by the clay or ferruginous oxides is separated by every shower of rain, and conveyed in solution to the soil.

Powdered charcoal possesses a similar action, but surpasses all other substances in the power which it possesses of condensing ammonia. within its pores, particularly when it has been previously heated to redness. Charcoal absorbs 90 times its volume of ammoniacal gas, which may be again separated by simply moistening it with water. (De Saussure.) Decayed wood approaches very nearly to charcoal in this power; decayed oak wood absorbs 72 times its volume, after having been completely dried under the air-pump.* We have here an easy and satisfactory means of explaining still further the properties of humus, or wood in a decaying state. It is not only a slow and constant source of carbonic acid, but it is also a means by which the necessary nitrogen is conveyed to plants.

Nitrogen is found in lichens, which grow on basaltic rocks. Our fields produce more of it than we have given them as manure, and it exists in all kinds of soils and minerals which were never in contact with organic substances. The nitrogen in these cases could only have been extracted from the atmosphere.

We find this nitrogen in the atmosphere in rain water and in all kinds of soils, in the form of ammonia, as a product of the decay and putrefaction of preceding generations of animals and vegetables. We find likewise that the proportion of azotized matters in plants is augmented by giving them a larger supply of ammonia conveyed in the form of animal manure.

No conclusion can then have a better foundation

* In experiments instituted by Dr. Daubeny, with a view of ascertaining whether vegetable mould had not the same property, he found that both carbonic acid and ammoniacal gases were condensed within its pores, as they would be by a lump of charcoal. 
than this, that it is the ammonia of the atmosphere which furnishes nitrogen to plants.*

Carbonic acid, water, and ammonia, contain the elements necessary for the support of animals and vegetables. The same substances are the ultimate products of the chemical processes of decay and putrefaction. All the innumerable products of vitality resume, after death, the original form from which they sprung. And thus death, - the complete dissolution of an existing generation, - becomes the source of life for a new one.

\section{CHAPTER VI.}

OF THE INORGANIC CONSTITUENTS OF PLANTS.

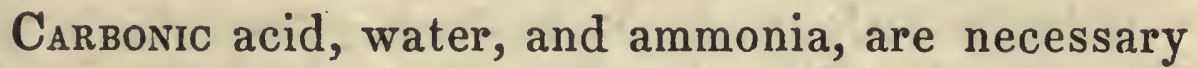
for the existence of plants, because they contain the elements from which their organs are formed; but other substances are likewise requisite for the formation of certain organs destined for special functions peculiar to each family of plants. Plants obtain these substances from inorganic nature. In the ashes left after the incineration of plants, the same substances are found, although in a changed condition.

Although the vital principle exercises a great power over chemical forces, yet it does so only by directing the way in which they are to act, and not by changing the laws to which they are subject. Hence when the chemical forces are employed in the processes of vegetable nutrition, they must produce the same results which are observed in ordinary chemical phenomena. The inorganic matter contained in plants

* From some experiments with respect to the action of light upon plants, Dr. Daubeny is inclined to suspect that in some cases hydrogen is assimilated whilst nitrogen is disengaged. See his Memoir in Philos. Trans. 1836. 
must, therefore, be subordinate to the laws which regulate its combinations in common chemical processes.

The most important division of inorganic substances is that of acids and alkalies. Both of these have a tendency to unite together, and form neutral compounds, which are termed salts. According to the doctrine of equivalents, these combinations are always effected in definite proportions, that is to say, one equivalent of an acid always unites with one or two equivalents of a base, whatever that base may be. Thus 501.17 parts by weight of sulphuric acid unite with 1 eq. of potash, and form 1 eq. of sulphate of potash; the same quantity unites with 1 eq. of soda, and produces sulphate of soda. From this fact follows the rule, - that the quantity, which an acid requires of an alkali for its saturation, may be represented by a very simple number.

It is perfectly necessary to form a proper conception of what chemists denominate the "capacity for saturation of an acid," before we are able to form a correct idea of the functions performed in plants, by their inorganic constituents. The power of a base to neutralize an acid does not depend upon the quantity of radical which it contains, but altogether upon the quantity of its oxygen. Thus protoxide of iron contains 1 eq. of oxygen, and unites with 1 eq. of sulphuric acid in forming a neutral salt; but peroxide of iron contains 3 eq. of oxygen, and requires 3 eq. of the same acid for its neutralization. Hence when a given weight of an acid is neutralized by different bases, the quantity of oxygen contained in these bases must be the same as is exhibited by the following scale:-

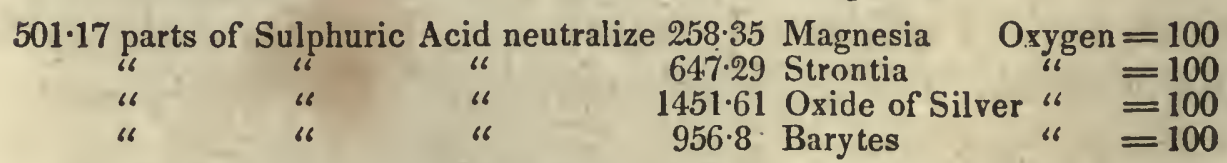

It follows from the law of equivalents, that the quantity of oxygen in a base must stand in a simple relation to the quantity of oxygen in an acid which 
unites with it. By this is meant, that the quantities in both cases must either be equal or multiples of each other; for the doctrine of equivalents denies the possibility of their uniting in fractional parts. This will be rendered obvious by a consideration of the two following examples:

100 parts of Cyanic Acid contain 2326 oxygen =1.

100 parts of Cyanic Acid saturate 137.21 parts of potash, which contain 2:3.26 oxygen $=1$.

100 parts of Nitric Acid contain 73.85 oxygen $=5$.

100 parts of Nitric Acid saturate 214.40 parts of oxide of silver, which contain 14.77 oxygen $=1$.

In the first of these cases, the relation of the oxygen of the base to that of the acid is as $1: 1$; in the second, as 1:5. The capacity for saturation of each acid is, therefore, the constant quantity of oxygen necessary to neutralize 100 parts of it.

Many of the inorganic constituents vary according to the soil in which the plants grow, but a certain number of them are indispensable to their development. All substances in solution in a soil are absorbed by the roots of plants, exactly as a sponge imbibes a liquid, and all that it contains, without selection. The substances thus conveyed to plants are retained in greater or less quantity, or are entirely separated when not suited for assimilation.

Phosphate of magnesia in combination with ammonia is an invariable constituent of the seeds of all kinds of grasses. It is contained in the outer horny husk, and is introduced into bread along with the flour, and also into beer. The bran of flour contains the greatest quantity of it. It is this salt which forms large crystalline concretions, often amounting to several pounds in weight, in the cacum of horses belonging to millers; and when ammonia is mixed with beer, the same salt separates as a white precipitate.

Most plants, perhaps all of them, contain organic acids of very different composition and properties, all of which are in combination with bases, such as 
potash, soda, lime, or magnesia. These bases evidently regulate the formation of the acids, for the diminution of the one is followed by a decrease of the other: thus in the grape, for example, the quantity of potash contained in its juice is less when it is ripe than when unripe; and the acids, under the same circumstances, are found to vary in a similar manner. Such constituents exist in small quantity in those parts of a plant in which the process of assimilation is most active, as in the mass of woody fibre; and their quantity is greater in those organs whose office it is to prepare substances conveyed to them for assimilation by other parts. 'The leaves contain more inorganic matters than the brancies, and the branches more than the stem. The potato plant contains more potash before blossoming than after it.

The acids found in the different families of plants are of various kinds; it cannot be supposed that their presence and peculiarities are the result of accident. 'The fumaric and oxalic acids in the liverwort, the kinovic acid in the China nova, the rocellic acid in the Rocella tinctoria, the tartaric acid in grapes, and the numerous other organic acids, must serve some end in vegetable life. But if these acids constantly exist in vegetables, and are necessary to their life, which is incontestable, it is equally certain that some alkaline base is also indispensable, in order to enter into combination with the acids which are always found in the state of salts. All plants yield by incineration ashes containing carbonic acid; all therefore must contain salts of an organic acid.*

Now, as we know the capacity of saturation of organic acids to be unchanging, it follows that the quantity of the bases united with them cannot vary, and for this reason the latter substances ought to

* Salts of organic acids yield carbonates on incineration, if they contain either alkaline or earthy bases. 
be considered with the strictest attention both by the agriculturist and physiologist.

We have no reason to believe that a plant in a condition of free and unimpeded growth produces more of its peculiar acids than it requires for its own existence; hence, a plant, on whatever soil it grows, must contain an invariable quantity of alkaline bases. Culture alone will be able to cause a deviation.

In order to understand this subject clearly, it will be necessary to bear in mind that any one of the alkaline bases may be substituted for another, the action of all being the same. Our conclusion is therefore by no means endangered by the existence of a particular alkali in one plant, which may be absent in others of the same species. If this inference be correct, the absent alkali or earth must be supplied by one similar in its mode of action, or in other words, by an equivalent of another base. The number of equivalents of these various bases which may be combined with a certain portion of acid must necessarily be the same, and therefore the amount of oxygen contained in them must remain unchanged under all circumstances and on whatever soil they grow.

Of course, this argument refers only to those alkaline bases which in the form of organic salts. form constituents of the plants. Now, these salts are preserved in the ashes of plants as carbonates, the quantity of which can be easily ascertained.

It has been distinctly shown, by the analysis of De Saussure and Berthier, that the nature of a soil exercises a decided influence on the quantity of the different metallic oxides contained in the plants which grow on it; that magnesia, for example, was contained in the ashes of a pine-tree grown at Mont Breven, whilst it was absent from the ashes of a tree of the same species from Mont La Salle, and that even the proportion of lime and potash was: very different. 
Hence it has been concluded, (erroneously, I believe,) that the presence of bases exercises no particular influence upon the growth of plants: but even were this view correct, it must be considered as a most remarkable accident that these same analyses furnish proof for the very opposite opinion. For although the composition of the ashes' of these pine-trees - was so very different, they contained, according to the analyses of De Saussure, an equal number of equivalents of metallic oxides; or, what is the same thing, the quantity of oxygen contained in all the bases was in both cases the same.

100 parts of the ashes of the pine-tree from Mont Breven contained -

Carbonate of Potash . 3.60 Quantity of oxygen in the Potash 0.41

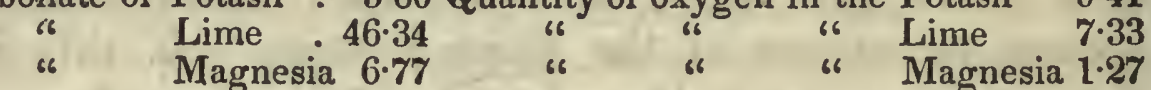

Sum of the carbonates $\overline{56 \cdot 71}$ Sum of the oxygen in the bases $\overline{9 \cdot 01}$ 100 parts of the ashes of the pine from Mont La Salle contained ${ }^{*}$ -

Carbonate of Potash . 7.36 Quantity of oxygen in the Potash 0.85

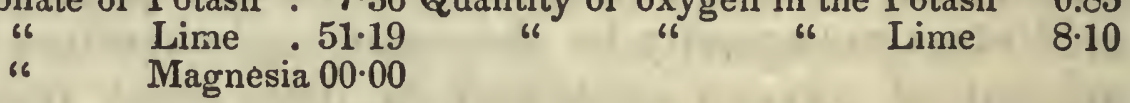

Sum of the carbonates $\overline{58.55}$ Sum of the oxygen in the bases $\overline{8.95}$

The numbers 9.01 and 8.95 resemble each other as nearly as could be expected even in analyses made for the very purpose of ascertaining the fact above demonstrated, which the analyst in this case had not in view.

Let us now compare Berthier's analyses of the ashes of two fir-trees, one of which grew in Norway, the other in Allevard (départment de l'Isère). One contained 50, the other 25 per cent. of soluble salts. A greater difference in the proportion of the alkaline bases could scarcely exist between two totally dif-

\footnotetext{
* According to the experiments of Saussure, 1000 parts of the wood of the pine from Mont Breven gave 11.87 parts of ashes; the same quantity of wood from Mont La Salle yielded 11.28 parts. From this we might conclude that the two pines, although brought up in different soils, yet contained the same quantity of inorganic elements.
} 
ferent plants, and yet even here the quantity of oxygen in the bases of both was the same.

100 parts of the ashes of firwood from Allevard contained, according to Berthier, (Ann. de Chim. et de Phys. t. xxxii. p. 248,)

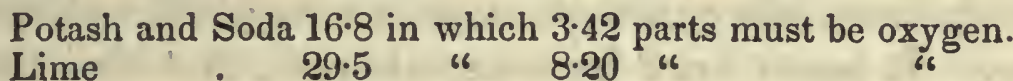

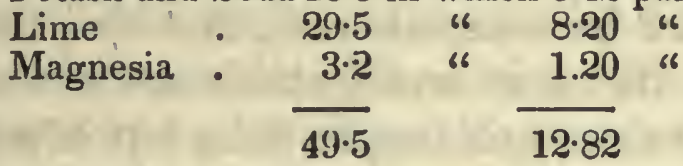

Only part of the potash and soda in these ashes was in combination with organic acids; the remainder was in the form of sulphates, phosphates, and chlorides. One hundred parts of the ashes contain 3.1 sulphuric acid, $4 \cdot 2$ phosphoric acid, and $0.3 \mathrm{hy}-$ drochloric acid, which together neutralize a quantity of base containing 1.20 oxygen. This number therefore must be subtracted from $12 \cdot 82$. The remainder 11.62 indicates the quantity of oxygen in the alkaline bases, combined with organic acids in the firwood of Allevard.

The firwood of Norway contained in 100 parts, *-

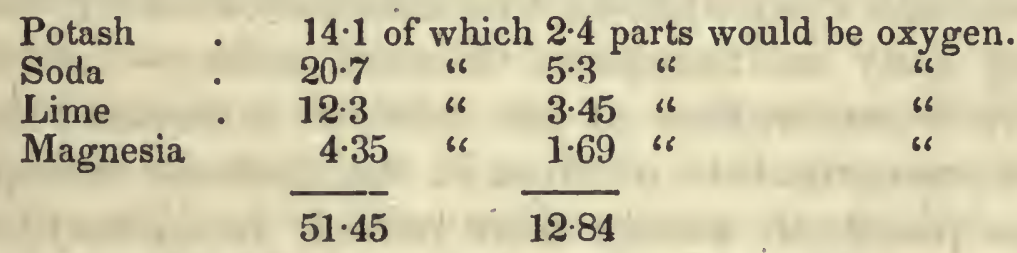

And if the quantity of oxygen of the bases in combination with sulphuric and phosphoric acid, viz. 1.37 , be again subtracted from $12.84,11.47$ parts remain as the amount of oxygen contained in the bases which were in combination with organic acids.

These remarkable approximations cannot be accidental; and if further examinations confirm them in other kinds of plants, no other explanation than that already given can be adopted.

* This calculation is exact only in the case where the quantity of ashes is equal in weight for a given quantity of wood; the difference cannot, however, be admitted to be so great as to change sensibly the above proportions. Berthier has not mentioned the proportion of ashes contained in the wood. 
It is not known in what form silica, manganese, and oxide of iron, are contained in plants; but we are certain that potash, soda, and magnesia, can be extracted from all parts of their structure in the form of salts of organic acids. The same is the case with lime, when not present as insoluble oxalate of lime. It must here be remembered, that in plants yielding oxalic acid, the acid and potash never exist in the form of a neutral or quadruple salt, but always as a double acid salt, on whatever soil they may grow. The potash in grapes also, is more frequently found as an acid salt, viz. cream of tartar (bitartrate of potash), than in the form of a neutral compound. As these acids and bases are never absent from plants, and as even the form in which they present themselves is not subject to change, it may be affirmed that they exercise an important influence on the development of the fruits and seeds, and also on many other functions of the nature of which we are at present ignorant.

'The quantity of alkaline bases existing in a plant also depends evidently on this circumstance of their existing only in the form of acid salts, - for the capacity of saturation of an acid is constant; and when we see oxalate of lime in the lichens occupying the place of woody fibre which is absent, we must regard it as certain that the soluble organic salts are destined to fulfil equally important though different functions, so much so that we could not conceive the complete development of a plant without their presence, that is, without the presence of their acids, and consequently of their bases.

From these considerations we must perceive, that exact and trustworthy examinations of the ashes of plants of the same kind growing upon different soils would be of the greatest importance to vegetable physiology, and would decide whether the facts above mentioned are the results of an unchanging law for each family of plants, and whether an invariable number can be found to express the quantity 
of oxygen which each species of plant contains in the bases united with organic acids. In all probability such inquiries will lead to most important results; for it is clear that if the production of a certain unchanging quantity of an organic acid is required by the peculiar nature of the organs of a plant, and is necessary to its existence, then potash or lime must be taken up by it in order to form salts with this acid; that if these do not exist in sufficient quantity in the soil, other bases must supply their place; and that the progress of a plant must be wholly arrested when none are present.

Seeds of the Salsola Kali, when sown in common garden soil, produce a plant containing both potash and soda; while the plants grown from the seeds of this contain only salts of potash, with mere traces of muriate of soda: (Cadet.)

The examples cited above, in which the quantity of oxygen contained in the bases was shown to be the same, lead us to the legitimate conclusion, that the development of certain plants is not retarded by the substitution of the bases contained in them. But it was by no means inferred that any one base could replace all the others, which are found in a plant in its normal condition. On the contrary, it is known that certain bases are indispensable for the growth of a plant, and these could not be substituted without injuring its development. Our inference has been drawn from certain plants, which can bear without injury this substitution; and it can only be extended to those plants which are in the same condition. It will be shown afterwards that corn or vines can only thrive on soils containing potash, and that this alkali is perfectly indispensable to their growth. Experiments have not been sufficiently multiplied so as to enable us to point out in what plants potash or soda may be replaced by lime or magriesia; we are only warranted in affirming that such substitutions are in many cases common. 'The ashes of various kinds of plants contain very differ- 
ent quantities of alkaline bases, such as potash, soda, lime, or magnesia. When lime exists in the ashes in large proportion, the quantity of magnesia is diminished, and in like manner according as the latter increases the lime or potash decreases. In many kinds of ashes not a trace of magnesia can be detected.

The existence of regetable alkalies in combination with organic acids gives great weight to the opinion, that alkaline bases in general are connected with the development of plants.

If potatoes are grown where they are not supplied with earth, the magazine of inorganic bases, (in cellạrs, for example,) a true alkali, called Solanin, of very poisonous nature, is formed in the sprouts which extend towards the light, while not the smallest trace of such a substance can be discovered in the roots, herbs, blossoms, or fruits of potatoes grown in fields. (Otto.)* In all the species of the Cinchona, kinic acid is found; but the quantity of quinia, cinchonina, and lime, which they contain is most variable. From the fixed bases in the products of incineration, however, we may estimate pretty accurately the quantity of the peculiar organic bases. A maximum of the first corresponds to a minimum of the latter, as must necessarily be the case if they mutually replace one another according to their equivalents. We know that different kinds of opium contain meconic acid in combination with very different quantities of narcotina, morphia, codeia, \&c., the quantity of one of these alkaloids diminishing on the increase of the others. Thus the smallest

* 'The analysis of potatoes afforded M. Henry

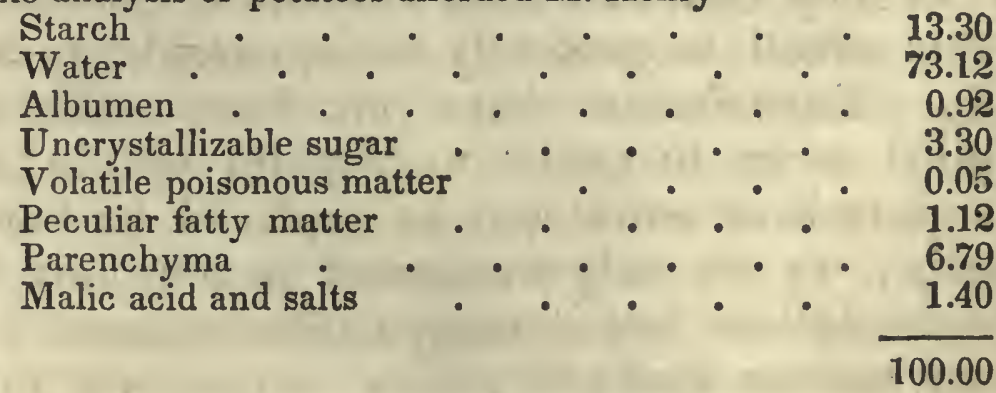


quantity of morphia is accompanied by a maximum of narcotina. Not a trace of meconic acid * can be discovered in many kinds of opium, but there is not on this account an absence of acid, for the meconic is here replaced by sulphuric acid. Here, also, we have an example of what has been before stated, for in those kinds of opium where both these acids exist, they are always found to bear a certain relative proportion to one another. Attention to these facts must be very important in the selection of soils destined for the cultivation of plants which yield the regetable alkaloids.

Now if it be found, as appears to be the case in the juice of poppies, that an organic acid may be replaced by an inorganic, without impeding the growth of a plant, we must admit the probability of this substitution taking place in a much higher degree in the case of the inorganic bases.

When roots find their more appropriate base in sufficient quantity, they will take up less of another.

'These phenomena do not show themselves so frequently in cultivated plants, because they are subjected to special external conditions for the purpose of the production of particular constituents or particular organs.

When the soil, in which a white hyacinth is growing in a state of blossom, is sprinkled with the juice of the Phytolacca decandra, the white blossoms assume in one or two hours a red color, which again disappears after a few days under the influence of sunshine, and they become white and colorless as before.t The juice in this case evidently enters into all parts of the plant, without being at all changed in its chemical nature, or without its presence being apparently either necessary or injurious. But this

* Robiquet did not obtain a trace of meconate of lime from $300 \mathrm{lbs}$. of opium, whilst in other kinds the quantity was very considerable. Ann. de Chim. liii. p. 425.

$\dagger$ American nightshade.

$\ddagger$ Biot, in the Comptes rendus des Séances de l'Académie des Sciences, d Paris, 1er Sémestre, 1837, p. 12. 
condition is not permanent, and when the blossoms have again become colorless, none of the coloring matter remains; and if it should occur that any of its elements were adapted for the purposes of nutrition of the plant, then these alone would be retained, whilst the rest would be excreted in an altered form by the roots.

Exactly the same thing must happen when we sprinkle a plant with a solution of chloride of potassium, nitre, or nitrate of strontia; they will enter into the different parts of the plant, just as the colored juice mentioned above, and will be found in its ashes if it should be burnt at this period. Their presence is merely accidental; but no conclusion can be hence deduced against the necessity of the presence of other bases in plants. The experiments of Macaire-Princep have shown, that plants made to vegetate with their roots in a weak solution of acetate of lead, and then in rain-water, yield to the latter all the salt of lead which they had previously absorbed. They return, therefore, to the soil all matters which are unnecessary to their existence. Again, when a plant, freely exposed to the atmosphere, rain, and sunshine, is sprinkled with a solution of nitrate of strontia, the salt is absorbed, but it is again separated by the roots and removed further from them by every shower of rain, which moistens the soil, so that at last not a trace of it is to be found in the plant.

Let us consider the composition of the ashes of two fir-trees as analyzed by an acute and most accurate chemist. One of these grew in Norway, on a soil the constituents of which never changed, but to which soluble salts, and particularly common salt, were conveyed in great quantity by rain-water. How did it happen that its ashes contained no appreciable trace of salt, although we are certain that its roots must have absorbed it after every shower?

We can explain the absence of salt in this case by means of the direct and positive observations refer- 
red to, which have shown that plants have the power of returning to the soil all substances unnecessary to their existence; and the conclusion to which all the foregoing facts lead us, when their real value and bearing are apprehended, is that the alkaline bases existing in the ashes of plants must be necessary to their growth, since if this were not the case they would not be retained.

The perfect development of a plant, according to this view, is dependent on the presence of alkalies or alkaline earths; for when these substances are totally wanting its growth will be arrested, and when they are only deficient it must be impeded.

In order to apply these remarks, let us compare two kinds of trees, the wood of which contains unequal quantities of alkaline bases, and we shall find that one of these grows luxuriantly in several soils upon which the others are scarcely able to vegetate. For example, 10,000 parts of oak-wood yield 250 parts of ashes, the same quantity of fir-wood only 83 , of linden-wood 500 , of rye 440 , and of the herb of the potato-plant 1500 parts.*

Firs and pines find a sufficient quantity of alkalies in granitic and barren sandy soils in which oaks will not grow; and wheat thrives in soils favorable for the linden-tree, because the bases which are necessary to bring it to complete maturity, exist there in sufficient quantity. The accuracy of these conclusions, so highly important to agriculture and to the cultivation of forests, can be proved by the most evident facts.

All kinds of grasses, the Equisetacea, for example, contain in the outer parts of their leaves and stalk a large quantity of silicic acid and potash in the form of acid silicate of potash. The proportion of this salt does not vary perceptibly in the soil of corn-fields, because it is again conveyed to them as manure in the form of putrefying straw. But this is

* Berthier, Annales de Chimic et de Physique, t. xxx. p. 248. 
not the case in a meadow', and hence we never find a luxuriant crop of grass * on sandy and calcareous soils, which contain little potash, evidently because one of the constituents indispensable to the growth of the plants is wanting. Soils formed from basalt, grauwacke, and porphyry, are, cateris paribus, the best for meadow-land, on account of the quantity of potash which enters into their composition. The potash abstracted by the plants is restored during the annual irrigation. The potash contained in the soil itself is inexhaustible in comparison with the quantity removed by plants. But when we increase the crop of grass in a meadow by means of gypsum, we remove a greater quantity of potash with the hay than can under the same circumstances be restored. Hence it happens that, after the lapse of several years, the crops of grass on the meadows manured with gypsum diminish, owing to the deficiency of potash. But if the meadow be strewed from time to time with wood-ashes, even with the lixiviated ashes which have been used by soap-boilers, (in Germany much soap is made from the ashes of wood,) then the grass thrives as luxuriantly as before. The ashes are only a means of restoring the potash. $\dagger$

* It would be of importance to examine what alkalies are contained in the ashes of the seashore plants which grow in the humid hollows of downs, and especially in those of the millet-grass. If potash is not found in them,-it must certainly be replaced by soda as in the Salsola, or by lime as in the Plumbaginea. $-L$.

$\dagger$ The compost which has been employed with most advantage as a top dressing to grass by Mr. Haggerston, on the estate of J. P. Cushing, Esq., at Watertown, is prepared from peat and barilla alone.

The peat previously cut and dried is made into heaps with alternate layers of barilla, the thickness of each layer of peat being eight inches, and of the barilla four inches. This heap is allowed to remain undisturbed during the winter, in the spring it is carefully turned and then allowed to remain until the ensuing autumn, when it is spread upon the land.

Peat which is to be ploughed into the land, having been deposited in the yard to which swine have free access, is mixed with stable manure in the proportion of two thirds peat to one third manure.

Barilla is the crude soda which is imported from Spain, Sicily, \&c., where it is prepared by burning the plant called salsolu soda. According to Dr. Ure it contains 20 per cent. of real alkali (soda) with muriates and sulphates of soda, some lime and alumina, with very little sulphur. 
A harvest of grain is obtained every thirty or forty years from the soil of the Luneburg heath, by strewing it with the ashes of the heath-plants (Erica vulgaris) which grow on it. These plants during the long period just mentioned collect the potash and soda, which are conveyed to them by rain-water; and it is by means of these alkalies that oats, barley, and rye, to which they are indispensable, are enabled to grow on this sandy heath.

The woodcutters in the vicinity of Heidelberg have the privilege of cultivating the soil for their own use, after felling the trees used for making tan. Before sowing the land thus obtained, the branches, roots, and leaves, are in every case burned, and the ashes used as a manure, which is found to be quite indispensable for the growth of the grain. The soil itself upon which the oats grow in this district consists of sandstone; and although the trees find in it a quantity of alkaline earths sufficient for their own sustenance, yet in its ordinary condition it is incapable of producing grain.

The most decisive proof of the use of strong manure was obtained at Bingen (a town on the Rhine), where the produce and development of vines were highly increased by manuring them with such substances as shavings of horn, \&c.; but after some years the formation of the wood and leaves decreased to the great loss of the possessor, to such a degree that he has long had cause to regret his departure from the usual methods. By the manure employed by him, the vines had been too much hastened in their growth; in two or three years they had exhausted the potash in the formation of their fruit, leaves, and wood, so that none remained for the future crops, his manure not having contained any potash.

There are vineyards on the Rhine the plants of which are above a hundred years old, and all of these have been cultivated by manuring them with cow-dung, a manure containing a large proportion 
of potash, although very little nitrogen. All the potash, in fact, which is contained in the food consumed by a cow is again immediately discharged in its excrements.

The experience of a proprietor of land in the vicinity of Gottingen offers a most remarkable example of the incapability of a soil to produce wheat or grasses in general, when it fails in any one of the materials necessary to their growth. In order to obtain potash, he planted his whole land with. wormwood, the ashes of which are well known to contain a large proportion of the carbonate of that alkali. The consequence was, that he rendered his land quite incapable of bearing grain for many years, in consequence of having entirely deprived the soil of its potash.

The leaves and small branches of trees contain the most potash; and the quantity of them which is annually taken from a wood, for the purpose of being employed as litter, ${ }^{*}$ contains more of that alkali than all the old wood which is cut down. The bark and foliage of oaks, for example, contain from 6 to 9 per cent. of this alkali; the needles of firs and pines, 8 per cent.

With every $2920 \mathrm{lbs}$. of firwood which are yearly removed from an acre of forest, only from 0.125 to $0.58 \mathrm{lbs}$. of alkalies are abstracted from the soil, calculating the ashes at 0.83 per cent. The moss, however, which covers the ground, and of which the ashes are known to contain so much alkali, continues uninterrupted in its growth, and retains that potash on the surface, which would otherwise so easily penetrate with the rain through the sandy soil. By its decay, an abundant provision of alkalies

* This refers to a custom some time since very prevalent in Germany, although now discontinued. The leaves and small twigs of trees were gleaned from the forests by poor people, for the purpose of being used as litter for their cattle. The trees, however, were found to suffer so much in consequence, that their removal is now strictly prohibited. The cause of the injury was that stated in the text. - Ev.] 
is supplied to the roots of the trees, and a fresh supply is rendered unnecessary.

'The supposition of alkalies, metallic oxides, or inorganic matter in general, being produced by plants, is entirely refuted by these well-authenticated facts.

It is thought very remarkable, that those plants of the grass tribe, the seeds of which furnish food for man, follow him like the domestic animals. But saline plants seek the seashore or saline springs, and the Chenopodium the dunghill from similar causes. Saline plants require common salt, and the plants which grow only on dunghills need ammonia and nitrates, and they are attracted whither these can be found, just as the dung-fly is to animal excrements. So likewise none of our corn-plants can bear perfect seeds, that is, seeds yielding flour, without a large supply of phosphate of magnesia and ammonia, substances which they require for their maturity. And hence, these plants grow only in a soil where these three constituents are found combined, and no soil is richer in them than those where men and animals dwell together; where the urine and excrements of these are found corn-plants appear, because their seeds cannot attain maturity unless supplied with the constituents of those matters.

When we find sea-plants near our salt-works, several hundired miles distant from the sea, we know that their seeds have been carried there in a very natural manner, namely, by wind or birds, which have spread them over the whole surface of the earth, although they grow only in those places in which they find the conditions essential to their life.

Numerous small fish, of not more than two inches in length (Gasterosteus aculeatus), are found in the salt-pans of the graduating house at Nidda (a village in Hesse Darmstadt). No living animal is found in the salt-pans of Neuheim, situated about 18 miles from Nidda; but the water there contains so much carbonic acid and lime, that the walls of the graduating house are covered with stalactites. Hence: 
the eggs conveyed to this place by birds do not find the conditions necessary for their development, which they found in the former place.*

How much more wonderful and inexplicable does it appear, that bodies which remain fixed in the strong heat of a fire, have under certain conditions the property of volatilizing, and, at ordinary temperatures, of passing into a state, of which we cannot say whether they have really assumed the form of a gas or are dissolved in one; Steam or vapors in general have a very singular influence in causing the volatilization of such bodies, that is, of causing them to assume the gaseous form. A liquid during evaporation communicates the power of assuming the same state in a greater or less degree to all substances dissolved in it, although they do not of themselves possess that property.

Boracic acid $\dagger$ is a substance which is completely fixed in the fire; it suffers no change of weight appreciable by the most delicate balance, when exposed to a white heat, and, therefore, it is not volatile. Yet its solution in water cannot be evaporated by the gentlest heat, without the escape of a sensible quantity of the acid with the steam. Hence it is that a loss is always experienced in the analysis of minerals containing this acid, when liquids in

* The itch-insect (Acarus Scabiei) is considered by Burdach as the production of a morbid condition, so likewise lice in children; the original generation of the fresh-water muscle (mytilus) in fish-ponds, of sea-plants in the vicinity of salt-works, of nettles and grasses, of fish in pools of rain, of trout in mountain streams, \&c., is according to the same natural philosopher not impossible. A soil consisting of crumbled rocks, decayed vegetables, rain and salt water, \&c., is here supposed to possess the power of generating shell-fish, trout, and saltwort (salicornia). All inquiry is arrested by such opinions, when propagated by a teacher who enjoys a merited reputation, obtained by knowledge and hard labor. These subjects, however, have hitherto met with the most superficial observation, although they well merit strict investigation. The dark, the secret, the mysterious, the enigmatic, is, in fact, too seducing for the youthful and philosophic mind, which would penetrate the deepest depths of nature, without the assistance of the shaft or ladder of the miner. This is poetry, but not sober philosophical inquiry.

+ The acid from borax. 
which it is dissolved are evaporated. The quantity of boracic acid which escapes with a cubic foot of steam, at the temperature of boiling water, cannot be detected by our most sensible re-agents; and nevertheless the many hundred tons annually brought from Italy as an article of commerce, are procured by the uninterrupted accumulation of this apparently inappreciable quantity. The hot steam which issues from the interior of the earth is allowed to pass through cold water in the lagoons of Castel Nuova and Cherchiago; in this way the boracic acid is gradually accumulated, till at last it may be obtained in crystals by the evaporation of the water. It is evident, from the temperature of the steam, that it must have come out of depths in which human beings and animals never could have lived, and yet it is very remarkable and highly important that ammonia is never absent from it. In the large works in Liverpool, where natural boracic acid is converted into borax, many hundred pounds of sulphate of ammonia are obtained at the same time.

This ammonia has not been produced by the animal organism, it existed before the creation of human beings; it is a part, a primary constituent, of the globe itself.*

The experiments instituted under Lavoisier's guidance by the Direction des Poudres et Saltpêtres, have proved that during the evaporation of the saltpetre ley, the salt volatilizes with the water, and causes a loss which could not before be explained. It is known also, that in sea-storms, leaves of plants in the direction of the wind are covered with crystals of salt, even at the distance of from 20 to 30 miles from the sea.t But it does not require a storm to cause the volatilization of the salt, for the air hanging over the sea always contains enough of this substance to make a solution of nitrate of silver turbid,

* See extract from Professor Daubeny's Lectures, in Appendix.

$\uparrow$ This was observed in the United States after the great storm of September 23, 1815. See Professor Farrar's account in Mem. A. A. S. 
and every breeze must carry this away. Now, as thousands of tons of sea-water annually evaporate into the atmosphere, a corresponding quantity of the salts dissolved in it, viz. of common salt, chloride of potassium, magnesia, and the remaining constituents of the sea-water, will be conveyed by wind to the land.

This volatilization is a source of considerable loss in salt-works, especially where the proportion of salt in the water is not large. This has been completely proved at the salt-works of Nauheim, by the very intelligent director of that establishment, $M$. Wilhelmi. He hung a plate of glass between two evaporating houses, which were about 1200 paces distant from each other, and found in the morning, after the drying of the dew, that the glass was covered with crystals of salt on one or the other side, according to the direction of the wind.

By the continual evaporation of the sea, its salts * are spread over the whole surface of the earth; and being subsequently carried down by the rain, furnish

* Analyses of sea-water.

Of the British Channel.

In 1000 parts. - MARCET.

$$
\text { - ScHWEITZER. }
$$

Water

Grs.

Chloride of Sodium

.... 964.74372

964.74372
27.05948

"6 of Potassium $\quad 1.232 \quad 0.76552$

"6 of Magnesium $\quad 5.152 \quad 3.66658$

Of the Mediterranean.

Bromide of Do.

0.02929

Sulphate of Soda

$$
\text { " of Magnesia }
$$

$$
4.660
$$

1.5

1.40662

- Laurens.

Grs.

95.9 .26

27.22

0.01

6.14

Carbonate of Lime

2.29578

0.15

According to $\mathrm{M}^{\prime} \mathrm{Clemm}$, the water of the North Sea contains in 1000 parts,

24.84 Chloride of Sodium.

2.42 Chloride of Magnesium.

2.06 Sulphate of Magnesia.

1.35 Chloride of Potassium.

1.20 Sulphate of Lime.

In addition to these constituents, it also contains inappreciable quantities of carbonate of lime, magnesia, iron, manganese, phosphate of lime, iodides and bromides, silica, sulphuretted hydrogen, and organic matter, together with ammonia and carbonic acid. (Liebig's Annalen der Chemie, Bd. xxxvii. s. 3.) 
to the vegetation those salts necessary to its existence. This is the origin of the salts found in the ashes of plants, in those cases where the soil could not have yielded them.

In a comprehensive view of the phenomena of nature, we have no scale for that which we are accustomed to name, small or great; all our ideas are proportioned to what we see around us, but how insignificant are they in comparison with the whole mass of the globe! that which is scarcely observable in a confined district appears inconceivably large when regarded in its extension through unlimited space. The atmosphere contains only a thousandth part of its weight of carbonic acid; and yet small as this proportion appears, it is quite sufficient to supply the whole of the present generation of living beings with carbon for a thousand years, even if it were not renewed. Sea-water contains $\frac{1}{12400}$ of its weight of carbonate of lime; and this quantity, although scarcely appreciable in a pound, is the source from which myriads of marine mollusca and corals are supplied with materials for their habitations.

Whilst the air contains only from 4 to 6 ten-thousandth parts of its volume of carbonic acid, seawater contains 100 times more, (10,000 volumes of sea-water contain 620 volumes of carbonic acidLaurent, Bouillon, Lagrange). Ammonia* is also found in this water, so that the same conditions which sustain living beings on the land are combined in this medium, in which a whole world of other plants and animals exists.

The roots of plants are constantly engaged in collecting from the rain those alkalies which formed part of the sea-water, and also those of the water of springs, which penetrates the soil. Without alkalies and alkaline bases most plants could not

* When the solid saline residue obtained by the evaporation of sea. water is heated in a retort to redness, a sublimate of sal-ammoniac is obtained. - Marcet. 
exist, and without plants the alkalies would disappear gradually from the surface of the earth.

When it is considered, that sea-water contains less than one-millionth of its own weight of iodine,* and that all combinations of iodine with the metallic bases of alkalies are highly soluble in water, some provision must necessarily be supposed to exist in the organization of sea-weed and the different kinds of Fuci, by which they are enabled during their life to extract iodine in the form of a soluble salt from. sea-water, and to assimilate it in such a manner, that it is not again restored to the surrounding medium. These plants are collectors of iodine, just as landplants are of alkalies; and they yield us this element, in quantities such as we could not otherwise obtain from the water without the evaporation of whole seas.

We take it for granted, that the sea-plants require metallic iodides $f$ for their growth, and that their existence is dependent on the presence of those substances. With equal justice, then, we conclude, that the alkalies and alkaline earths, always found in the ashes of land-plants, are likewise necessary for their development.

\section{CHAPTER VII.}

THE ART OF CULTURE.

THE conditions necessary for the life of all vegetables have been considered in the preceding part

* This substance was discovered in 1812 , and is obtained from marine plants; it is found also in sea-water and several mineral springs in combination with hydrogen, as hydriodic acid. With bases this acid forms hydriodates. Iodine has not been decomposed. It is a solid, and at about $350^{\circ} \mathrm{F}$. passes into vapor of a beautiful violet color; hence its name.

$\uparrow$ Compounds of metals and iodine. 
of the work. Carbonic acid, ammonia, and water yield elements for all the organs of plants. Certain inorganic substances - salts and metallic oxidesserve peculiar functions in their organism, and many of them must be viewed as essential constituents of particular parts.

The atmosphere and the soil offer the same kind of nourishment to the leaves and roots. The former contains a comparatively inexhaustible supply of carbonic acid and ammonia; the latter, by means of its humus, generates constantly fresh carbonic acid, whilst, during the winter, rain and snow introduce into the soil a quantity of ammonia, sufficient for the development of the leaves and blossoms.

The complete, or it may be said, the absolute insolubility in cold water of vegetable matter in progress of decay, (humus,) appears on closer consideration to be a most wise arrangement of nature. For if humus possessed even a smaller degree of solubility than that ascribed to the substance called humic acid, it must be dissolved by rain-water. Thus, the yearly irrigation of meadows, which lasts for several weeks, would remove a great part of it from the ground, and a heavy and continued rain would impoverish a soil. But it is soluble only when combined with oxygen; it can be taken up by water, therefore, only as carbonic acid.

When kept in a dry place, humus may be preserved for centuries; but when moistened with water, it converts the surrounding oxygen into carbonic acid. As soon as the action of the air ceases, that is, as soon as it is deprived of oxygen, the humus suffers no further change. Its decay proceeds only when plants grow in the soil containing it; for they absorb by their roots the carbonic acid as it is formed. The soil receives again from living plants the carbonaceous matter it thus loses, so that the proportion of humus in it does not decrease.

The stalactitic caverns in Franconia, and those in the vicinity of Baireuth, and Streitberg, lie beneath 
a fertile arable soil; the abundant decaying vegetables or humus in this soil, being acted on by moisture and air, constantly evolve carbonic acid, which is dissolved by the rain. The rain-water thus impregnated permeates the porous limestone, which forms the walls and roofs of the caverns, and dissolves in its passage as much carbonate of lime as corresponds to the quantity of carbonic acid contained in it. Water and the excess of carbonic acid evaporate from this solution when it has reached the interior of the caverns, and the limestone is deposited on the walls and roofs in crystalline crusts of various forms. There are few spots on the earth where so many circumstances favorable to the production of humate of lime are combined, if the humus actually existed in the soil in the form of humic acid. Decaying vegetable matter, water, and lime in solution, are brought together, but the stalactites formed contain no trace of vegetable matter, and no humic acid; they are of a glistening white or yellowish color, and in part transparent, like calcareous spar, and may be heated to redness without becoming black.

The subterranean vaults in the old castles near the Rhine, the "Bergstrass," and Wetherau, are constructed of sandstone, granite, or basalt, and present appearances similar to the limestone caverns. The roofs of these vaults or cellars are covered externally to the thickness of several feet with vegetable mould, which has been formed by the decay of plants. The rain falling upon them sinks through the earth, and dissolves the mortar by means of the carbonic acid derived from the mould; and this solution evaporating in the interior of the vaults, covers them with small thin stalactites, which are quite free from humic acid.

In such a filtering apparatus, built by the hand of nature, we have placed before us experiments which have been continued for a hundred or a thousand years. Now, if water possessed the power of dis- 
solving a hundred-thousandth part of its own weight of humic acid or humate of lime, and humic acid were present, we should find the inner surface of the roofs of these vaults and caverns covered with these substances; but we cannot detect the smallest trace of them. There could scarcely be found a more clear and convincing proof of the absence of the humic acid of chemists in common vegetable mould.

The common view, which has been adopted respecting the modus operandi of humic acid, does not afford any explanation of the following phenomenon :- A very small quantity of humic acid dissolved in water gives it a yellow or brown color. Hence it would be supposed that a soil would be more fruitful in proportion as it was capable of giving this color to water, that is, of yielding it humic acid. But it is very remarkable that plants do not thrive in such a soil, and that all manure must have lost this property before it can exercise a favorable influence upon their vegetation. Water from barren peat soils and marshy meadows, upon which few plants flourish, contains much of this humic acid; but all agriculturists and gardeners agree that the most suitable and best manure for plants is that which has completely lost the property of giving a color to water.

-The soluble substance, which gives to water a brown color, is a product of the putrefaction of all animal and vegetable matters; its formation is an evidence that there is not oxygen sufficient to begin, or at least to complete the decay. The brown solutions containing this substance are decolorized in the air by absorbing oxygen, and a black coaly matter precipitates - the substance named "coal of humus." Now if a soil were impregnated with this matter, the effect on the roots of plants would be the same as that of entirely depriving the soil of oxygen; plants would be as little able to grow in such ground as they would if hydrated protoxide of iron were mixed with the soil. Indeed, some 
barren soils have been found to owe their sterility to this very cause. The sulphate of protoxide of iron (copperas), which forms a constituent of these soils, possesses a powerful affinity for oxygen, and consequently prevents the absorption of that gas by the roots of plants in its vicinity.* All plants die in soils and water which contain no oxygen; absence of air acts exactly in the same manner as an excess of carbonic acid. Stagnant water on a marshy soil excludes air, but a renewal of water has the same effect as a renewal of air, because water contains it in solution. If the water is withdrawn from a marsh, free access is given to the air, and the marsh is changed into a fruitful meadow.

In a soil to which the air has no access, or at most but very little, the remains of animals and vegetables do not decay, for they can only do so when freely supplied with oxygen; but they undergo putrefaction, for which air is present in sufficient quantity. Putrefaction is known to be a most powerful deoxidizing process, the influence of which extends to all surrounding bodies, even to the roots and the plants themselves. All substances from which oxygen can be extracted yield it to putrefying bodies; yellow oxide of iron passes into the state of black oxide, sulphate of iron into sulphuret of iron, \&c.

The frequent renewal of air by ploughing, and the preparation of the soil, especially its contact with alkaline metallic oxides, the ashes of brown coal, burnt lime or limestone, change the putrefaction of its organic constituents into a pure process of oxidation; and from the moment at which all the organic matter existing in a soil enters into a state of oxidation or decay, its fertility is increased. The oxygen is no longer employed for the conversion of

* The most obvious method of removing this salt from soils in which it may be contained is to manure the land with lime. The lime unites with the sulphuric acid and liberates the protoxide of iron, which absorbs oxygen with much rapidity, and is converted into the peroxide of iron. This conversion is accelerated by giving free access to the air, that is, by loosening the soil. 
the brown soluble matter into the insoluble coal of humus, but serves for the formation of carbonic acid. This change takes place very slowly, and in some instances the oxygen is completely excluded by it; and whenever this happens, the soil loses its fertility. Thus, in the vicinity of Salzhausen (a village in Hesse Darmstadt, famed for its mineral springs,) upon a meadow called Grünschwalheimer; unfruitful spots are seen here and there covered with a yellow grass. If a hole be bored from twenty to twenty-five feet deep in one of these spots, carbonic acid is emitted from it with such violence that the noise made by the escape of the gas may be distinctly heard at the distance of several feet. Here the carbonic acid rising to the surface displaces completely all the air, and consequently all the oxygen, from the soil; and without oxygen neither seeds nor roots can be developed; a plant will not vegetate in pure nitrogen or carbonic acid gas.*

Humus supplies young plants with nourishment by the roots, until their leaves are matured sufficiently to act as exterior organs of nutrition; its quantity heightens the fertility of a soil by yielding more nourishment in this first period of growth, and consequently by increasing the number of organs of atmospheric nutrition. Those plants which receive their first food from the substance of their seeds, such as bulbous plants, could completely dispense with humus; its presence is useful only in so far as it increases and accelerates their development, but it is not necessary,-indeed, an excess of it at the commencement of their growth is in a certain measure injurious.

The amount of food which young plants can take from the atmosphere in the form of carbonic acid and ammonia is limited; they cannot assimilate more than the air contains. Now, if the quantity of their stems, leaves, and branches has been increased by 
the excess of food yielded by the soil at the commencement of their development, they will require for the completion of their growth, and for the formation of their blossoms and fruits, more nourishment from the air than it can afford, and consequently they will not reach maturity. In many cases the nourishment afforded by the air under these circumstances suffices only to complete the formation of the leaves, stems, and branches. The same result then ensues as when ornamental plants are transplanted from the pots in which they have grown to larger ones, in which their roots are permitted to increase and multiply. All their nourishment is employed for the increase of their roots and leaves; they spring, as it is said, into an herb or weed, but do not blossom. When, on the contrary, we take away part of the branches, and of course their leaves with them, from dwarf trees, since we thus prevent the development of new branches, an excess of nutriment is artificially procured for the trees, and is employed by them in the increase of the blossoms and enlargement of the fruit. It is to effect this purpose that vines are pruned.

A new and peculiar process of vegetation ensues in all perennial plants, such as shrubs, fruit and forest trees, after the complete maturity of their fruit. The stem of annual plants at this period of their growth becomes woody, and their leaves change in color. The leaves of trees and shrubs, on the contrary, remain in activity until the commencement of the winter. The formation of the layers of wood progresses, the wood becomes harder and more.solid, but after August the leaves form no more wood; all the carbonic acid which the plants now absorb is employed for the production of nutritive matter for the following year: instead of woody fibre, starch is formed, and is diffused through every part of the plant by the autumnal sap (sève d'Août).* Ac-

* Hartig, in Erdmann und Schweigger-Seidels Journal, V. 217. 1835. 
cording to the observations of M. Heyer, the starch thus deposited in the body of the tree can be recognised in its known form by the aid of a good microscope. The barks of several aspens and pine-trees * contain so much of this substance, that it can be extracted from them as from potatoes by trituration with water. It exists also in the roots and other parts of perennial plants. A very early winter, or sudden change of temperature, prevents the formation of this provision for the following year; the wood, as in the case of the vine-stock, does not ripen, and its growth is in the next year very limited.

From the starch thus accumulated, sugar and gam are produced in the succeeding spring, while from the gum those constituents of the leaves and young sprouts which contain no nitrogen are in their turn formed. After potatoes have germinated, the quantity of starch in them is found diminished. 'The juice of the maple-tree ceases to be sweet from the loss of its sugar when its buds, blossoms, and leaves attain their maturity.

The branch of a willow, which contains a large quantity of granules of starch in every part of its woody substance, puts forth both roots and leaves in pure distilled rain-water; but in proportion as it

* It is well known that bread is made from the bark of pines in Sweden during famines.

The following directions are given by Professor Autenrieth for preparing a palatable and nutritious bread from the beech and other woods destitute of turpentine. Every thing soluble in water is first removed by frequent maceration and boiling, the wood is then to be reduced to a minute state of division, not merely into fine fibres, but actual powder; and after being repeatedly subjected to heat in an oven, is ground in the usual manner of corn. Wood thus prepared, according to the author, acquires the smell and taste of corn flour. It is, however, never quite white. It agrees with corn flour in not fermenting without the addition of leaven, and in this case some leaven of corn flour is found to answer best. With this it makes a perfectily uniform and spongy bread; and when it is thoroughly baked, and has much crust, it has a much better taste of bread than what in time of scarcity is prepared from the bran and husks of corn. Wood-flour also, boiled in water, forms a thick, tough, trembling jelly, which is very nutritious. - Philosophical Transactions, 1827. 
grows, the starch disappears, it being evidently exhausted for the formation of the roots and leaves. In the course of these experiments, M. Heyer made the interesting observation, that such branches when placed in snow-water (which contains ammonia) produced roots three or four times longer than those which they formed in pure distilled water, and that this pure water remained clear, while the rain-water gradually acquired a yellow color.

Upon the blossoming of the sugar-cane, likewise, part of the sugar disappears; and it has been ascertained, that the sugar does not accumulate in the beet-root until after the leaves are completely formed.

Much attention has recently been drawn to the fact that the produce of potatoes may be much increased by plucking off the blossoms from the plants producing them, a result quite consistent with theory. This important observation has been completely confirmed by M. Zeller, the director of the Agricultural Society at Darmstadt. In the year 1839, two fields of the same size, lying side by side and manured in the same manner, were planted with potatoes. When the plants had flowered, the blossoms were removed from those in one field, while those in the other field were left untouched. The former produced 47 bolls, the latter only 37 bolls.

These well-authenticated observations remove every doubt as to the part which sugar, starch, and gum play in the development of plants; and it ceases to be enigmatical, why these three substances exercise no influence on the growth or process of nutrition of a matured plant, when supplied to them as food.

The accumulation of starch in plants during the autumn has been compared, although certainly erroneously, to the fattening of hibernating animals before their winter sleep; but in these animals every vital function, except the process of respiration, is suspended, and they only require, like a lamp slowly burning, a substance rich in carbon and hydrogen to 
support the process of combustion in the lungs. On their awaking from their torpor in the spring, the fat has disappeared, but has not served as nourishment. It has not caused the least increase in any part of their body, neither has it changed the quality of any of their organs. With nutrition, properly so called, the fat in these animals has not the least connexion.

The annual plants form and collect their future nourishment in the same way as the perennial; they store it in their seeds in the form of vegetable albumen, starch and gum, which are used by the germs for the formation of their leaves and first radicle fibres. The proper nutrition of the plants, their increase in size, begins after these organs are formed.

Every germ and every bud of a perennial plant is the engrafted embryo of a new individual, while the nutriment accumulated in the stem and roots, corresponds to the albumen of the seeds.

Nutritive matters are, correctly speaking, those substances which, when presented from without, are capable of sustaining the life and all the functions of an organism, by furnishing to the different parts of plants the materials for the production of their peculiar constituents.

In animals, the blood is the source of the material of the muscles and nerves; by one of its component parts, the blood supports the process of respiration, by others, the peculiar vital functions ; every part of the body is supplied with nourishment by it, but its own production is a special function, without which we could not conceive life to continue. If we destroy the activity of the organs which produce it, or if we inject the blood of one animal into the veins of another, at all events, if we carry this beyond certain limits, death is the consequence.

If we could introduce into a tree woody fibre in a state of solution, it would be the same thing as placing a potato plant to vegetate in a paste of starch. The office of the leaves is to form'starch, woody fibre, and sugar; consequently, if we convey these sub- 
stances through the roots, the vital functions of the leaves must cease, and if the process of assimilation cannot take another form, the plant must die.

Other substances must be present in a plant, besides the starch, sugar, and gum, if these are to take part in the development of the germ, leaves, and first radicle fibres. There is no doubt that a grain of wheat contains within itself the component parts of the germ and of the radicle fibres, and, we must suppose, exactly in the proportion necessary for their. formation. These component parts are starch and gluten; and it is evident that neither of them alone, but that both simultaneously assist in the formation of the root, for they both suffer changes under the action of air, moisture, and a suitable temperature. The starch is converted into sugar, and the gluten also assumes a new form, and both acquire the capability of being dissolved in water, and of thus being conveyed to every part of the plant. Both the starch and the gum are completely consumed in the formation of the first part of the roots and leaves; an excess of either could not be used in the formation of leaves, or in any other way.

The conversion of starch into sugar during the germination of grain is ascribed to a vegetable principle called diastase, which is generated during the act of commencing germination. But this mode of transformation can also be effected by gluten, although it requires a longer time. Seeds, which have germinated, always contain much more diastase than is necessary for the conversion of their starch into sugar, for five parts by weight of starch can be converted into sugar by one part of malted barley. This excess of diastase can by no means be regarded as accidental, for, like the starch, it aids in the formation of the first organs of the young plant; and disappears. with the sugar; diastase contains nitrogen and furnishes the elements of vegetable albumen.

Carbonic acid, water, and ammonia, are the food of fully-developed plants; starch, sugar, and gum, 
serve, when accompanied by an azotized substance, to sustain the embryo, until its first organs of nutrition are unfolded. The nutrition of a fœtus and development of an egg proceed in a totally different manner from that of an animal which is separated from its parent; the exclusion of air does not endanger the life of the fœtus, but would certainly cause the death of the independent animal. In the same manner, pure water is more advantageous to the growth of a young plant, than that containing carbonic acid, but after a month the reverse is the case.

The formation of sugar in maple-trees does not take place in the roots, but in the woody substance of the stem. The quantity of sugar in the sap augments until it reaches a certain height in the stem of the plant, above which point it remains stationary.

Just as germinating barley produces a substance which, in contact with starch, causes it to lose its insolubility and to become sugar, so in the roots of the maple, at the commencement of vegetation, a substance must be formed, which, being dissolved in water, permeates the wood of the trunk, and converts into sugar the starch, or whatever it may be, which it finds deposited there. It is certain, that when a hole is bored into the trunk of a maple-tree just above its roots, filled with sugar, and then closed again, the sugar is dissolved by the ascending sap. It is further possible that this sugar may be disposed of in the same manner as that formed in the trunks; at all events it is certain, that the introduction of it does not prevent the action of the juice upon the starch, and since the quantity of the sugar present is now greater than can be exhausted by the leaves and buds, it is excreted from the surface of the leaves or bark. Certain diseases of trees, for example that called honey-dew, evidently depend on the want of the due proportion between the quantity of the azotized and that of the unazotized substances which are applied to them as nutriment. 
In whatever form, therefore, we supply plants with those substances which are the products of their own action, in no instance do they appear to have any effect upon their growth, or to replace what they have lost. Sugar, gum, and starch, are not food for plants, and the same must be said of humic acid, which is so closely allied to them in composition.

If now we direct our attention to the particular organs of a plant, we find every fibre and every particle of wood surrounded by a juice containing an azotized matter; while the starch, granules, and sugar, are enclosed in cells formed of a substance containing nitrogen. Indeed everywhere, in all the juices of the fruits and blossoms, we find a substance destitute of nitrogen, accompanied by one which contains that element.

The wood of the stem cannot be formed, quasi wood, in the leaves, but another substance must be produced which is capable of being transformed into wood. This substance must be in a state of solution, and accompanied by a compound containing nitrogen; it is very probable that the wood and the vegetable gluten, the starch granules and the cells containing them, are formed simultaneously, and in this case a certain fixed proportion between them would be a condition necessary for their production.

According to this view, the assimilation of the substances generated in the leaves will (cateris paribus) depend on the quantity of nitrogen contained in the food. When a sufficient quantity of nitrogen is not present to aid in the assimilation of the substances which do not contain it, these substances will be separated as excrements from the bark, roots, leaves, and branches. The exudations of mannite, gum, and sugar, in strong and healthy plants cannot be ascribed to any other cause.*

* M. Trapp in Giessen possesses a Clerodendron fragrans, which grows in the house, and exudes on the surface of its leaves in September large colorless drops of sugar-candy, which form regular crystals upon drying; - I am not aware whether the juice of this plant 
Analogous phenomena are presented by the process of digestion in the human organism. In order that the loss which every part of the body sustains by the processes of respiration and perspiration may be restored to it, the organs of digestion require to be supplied with food, consisting of substances containing nitrogen, and of others destitute of it, in definite proportions. If the substances which do not contain nitrogen preponderate, either they will be expended in the formation of fat, or they will pass unchanged through the organism. This is particularly observed in those people who live almost exclusively upon potatoes; their excrements contain a large quantity of unchanged granules of starch, of which no trace can be detected when gluten or flesh is taken in proper proportions, because in this case the starch has been rendered capable of assimilation. Potatoes, which when mixed with hay alone are scarcely capable of supporting the strength of a horse, form with bread and oats a strong and wholesome fodder.

It will be evident from the preceding considerations, that the products generated by a plant may vary exceedingly, according to the substances given it as food. A superabundance of carbon in the state of carbonic acid conveyed through the roots of plants, without being accompanied by nitrogen, cannot be converted either into gluten, albumen, wood, or any other component part of an organ; but either it will be separated in the form of excrements, such as sugar, starch, oil, wax, resin, mannite,* or gum, or these substances will be deposited in greater or less quantity in the wide cells and vessels.

contains sugar. Professor Redtenbacher, of Prague, informs me that he has analyzed the crystals, and found them to be perfectly pure sugar. - Ev.

Mannite forms the greater part of manna. It is found in the juices of several fruits, in the fermented juice of beet-root, carrots, onions, \&c.; it is also obtained in small quantity when starch is transformed into grape sugar by boiling with dilute sulphuric acid. It crystallizes in prisms, is faintly sweet, soluble in water and hot alcohol. Its aqueous solution cannot be made to undergo the vinous fermentation. Its formula is $\mathrm{C}_{6} \mathrm{H}_{7} \mathrm{O}_{6}$. 
The quantity of gluten, vegetable albumen, and mucilage, will augment when plants are supplied with an excess of food containing nitrogen; and ammoniacal salts will remain in the sap, when, for example, in the culture of the beet, we manure the soil with a highly nitrogenous substance, or when we suppress the functions of the leaves by removing them from the plant.

We know that the ananas is scarcely eatable in its wild state, and that it shoots forth a great quantity of leaves when treated with rich animal manure, without the fruit on that account acquiring a large amount of sugar; that the quantity of starch in potatoes increases when the soil contains much humus, but decreases when the soil is manured with strong animal manure, although then the number of cells increases, the potatoes acquiring in the first case a mealy, in the second a soapy, consistence. Beet-roots taken from a barren, sandy soil contain a maximum of sugar, and no ammoniacal salts; and the 'Teltowa parsnep loses its mealy state in a manured land; because there all the circumstances necessary for the formation of cells are united.*

An abnormal $\dagger$ production of certain component parts of plants presupposes a power and capability of assimilation to which the most powerful chemical action cannot be compared. The best idea of it may be formed by considering that it surpasses in power the strongest galvanic battery, with which we are not able to separate the oxygen from carbonic acid. The affinity of chlorine for hydrogen, and its power to decompose water under the influence of light

* Children fed upon arrow-root, salep, or indeed any kind of amylaceous food, which does not contain ingredients fitted for the formation of bones and muscles, become fat, and acquire much embonpoint; their limbs appear full, but, they do not acquire strength, nor are their organs properly developed. $-L$.

† Abnormal, (Lat. ab, from, and norma, a rule,) Any thing without, or contrary to, systern or rule. In botany, if a flower has five petals, the rule is, that it should have the same number of stamens, or some regular multiple of that number; if it has only four or six stamens, the flower is abnormal. 
and set at liberty its oxygen, cannot be considered as at all equalling the power and energy with which a leaf separated from a plant decomposes the carbonic acid which it absorbs.

The common opinion, that only the direct solar rays can effect the decomposition of carbonic acid in the leaves of plants, and that reflected or diffused light does not possess this property, is wholly an error, for exactly the same constituents are generated in a number of plants, whether the direct rays of the sun fall upon them, or whether they grow in the shade. They require light, and indeed sunlight, but it is not necessary that the direct rays of the sun reach them. Their functions certainly proceed with greater intensity and rapidity in sunshine than in the diffused light of day; but there is nothing more in this than the similar action which light exercises on ordinary chemical combinations; it merely accelerates in a greater or less degree the action already subsisting.

Thus chlorine * and hydrogen combining form muriatic (hydrochloric) acid. This combination is effected in a few hours in common daylight, but it ensues instantly, with a violent explosion, under exposure to the direct solar rays, whilst not the slightest change in the two gases takes place in perfect darkness. When the liquid hydrocarburet of chlorine, resulting from the union of the olefiant gas $\dagger$ of the associated

* Chlorine is a gas named from its green color; it was formerly called oxymuriatic acid. It has not been decomposed. It is one of the most 'suffocating of the gases, and highly irritating, even when much diluted with air. It is largely absorbed by water, and the solution has the property of bleaching. Its solution in water cannot be kept unchanged, as the chlorine unites to the hydrogen of the water and forms muriatic or hydrochloric acid.

Bleaching salts are formed by exposing lime to an atmosphere of chlorine. Chlorine is useful for removing offensive odors. A few table spoonfuls of bleaching powder, sprinkled occasionally in privies, and in larger quantities upon heaps of offensive substances, upon the floors of slaughter-houses, \&c. will destroy the unpleasant odor, and at the same time add to the value of the manure.

For description of chlorine, and the metlod of procuring it, see WEBSTER's Chemistry, 3d edit. p. 180.

$f$ One of the compounds of hydrogen and carbon. 
Dutch chemists with chlorine, is exposed in a vessel with chlorine gas to the direct solar rays, chloride of carbon is immediately produced; but the same compound can be obtained with equal facility in the diffused light of day, a longer time only being required. When this experiment is performed in the way first mentioned, two products only are observed (muriatic acid and perchloride of carbon); whilst by the latter method a class of intermediate bodies are produced, in which the quantity of chlorine constantly augments, until at last the whole liquid hydrocarburet of chlorine is converted into the same two products as in the first case. Here, also, not the slightest trace of decomposition takes place in the dark. Nitric acid is decomposed in common daylight into oxygen, and peroxide of nitrogen; and chloride of silver becomes black in the diffused light of day, as well as in the direct solar rays; - in short, all actions of a similar kind proceed in the same way in diffused light as well as in the solar light, the only difference consisting in the time in which they are effected. It cannot be otherwise in plants, for the mode of their nutriment is the same in all, and their component substances afford proof that their food has suffered absolutely the same change, whether they grow in the sunshine or in the shade.

All the carbonic acid, therefore, which we supply to a plant will undergo a transformation, provided its quantity be not greater than can be decomposed by the leaves. We know that an excess of carbonic acid kills plants, but we know also that nitrogen to a certain degree is not essential for the decomposition of carbonic acid. All the experiments hitherto instituted prove, that fresh leaves placed in water impregnated with carbonic acid, and exposed to the influence of solar light, emit oxygen gas, whilst the carbonic acid-disappears. Now in these experiments no nitrogen is supplied at the same time with the carbonic acid; hence no other conclusion can be drawn 
from them than that nitrogen is not necessary for the decomposition of carbonic acid, - for the exercise, therefore, of one of the functions of plants. And yet the presence of a substance containing this element appears to be indispensable for the assimilation of the products newly formed by the decomposition of the carbonic acid, and their consequent adaptation for entering into the composition of the different organs.

The carbon abstracted from the carbonic acid acquires in the leaves a new form, in which it is soluble and transferable to all parts of the plant. In this new form the carbon aids in constituting several new products; these are named sugar when they possess a sweet taste, gum or mucilage when tasteless, and excrementitious matters when expelled by the roots.

Hence it is evident, that the quantity and quality of the substances generated by the vital processes of a plant will vary according to the proportion of the different kinds of food with which it is supplied. The development of every part of a plant in a free and uncultivated state depends on the amount and nature of the food afforded to it by the spot on which it growis. A plant is developed on the most sterile and unfruitful soil as well as on the most luxuriant and fertile, the only difference which can be observed being in its height and size, in the number of its twigs, branches, leares, blossoms, and fruit. Whilst the individual organs of a plant increase on a fertile soil, they diminish on another where those substances which are necessary for their formation are not so bountifully supplied; and the proportion of the constituents which contain nitrogen and of those which do not in plants varies with the amount of nitrogenous matters in their food.

The development of the stem, leaves, blossoms, and fruit of plants is dependent on certain conditions, the knowledge of which enables us to exercise some influence on their internal constituents 
as well as on their size. It is the duty of the natural philosopher to discover what these conditions are; for the fundamental principles of agriculture must be based on a knowledge of them. There is no profession which can be compared in importance with that of agriculture, for to it belongs the production of food for man and animals; on it depends the welfare and development of the whole human species, the riches of states, and all commerce. There is no other profession in which the application of correct principles is productive of more beneficial effects, or is of greater and more decided influence. Hence it appears quite unaccountable, that we may vainly search for one leading principle in the writings of agriculturists and vegetable physiologists.

The methods employed in the cultivation of land are different in every country, and in every district; and when we inquire the causes of these differences, we receive the answer, that they depend upon circumstances. (Les circonstances font les assolements.) No answer could show ignorance more plainly, since no one has ever yet devoted himself to ascertain what these circumstances are. Thus also when we inquire in what manner manure acts, we are answered by the most intelligent men, that its action is covered by the veil of Isis; and when we demand further what this means, we discover merely that the excrements of men and animals are supposed to contain an incomprehensible something which assists in the nutrition of plants, and increases their size. This opinion is embraced without even an attempt being made to discover the component parts of manure, or to become acquainted with its nature.*

In addition to the general conditions, such as heat, light, moisture, and the component parts of the atmosphere, which are necessary for the growth of all plants, certain substances are found to exercise a

* This statement is now somewhat too general ; both in this country and in Great Britain agriculture has received important aid from the labors of chemists and physiologists. 
peculiar influence on the development of particular families. These substances either are already contained in the soil, or are supplied to it in the form of the matters known under the general name of manure. But what does the soil contain, and what are the components of the substances used as manure? Until these points are satisfactorily determined, a rational system of agriculture cannot exist. The power and knowledge of the physiologist, of the agriculturist and chemist, must be united for the complete solution of these questions; and in order to attain this end, a commencement must be made.

The general object of agriculture is to produce in the most advantageous manner certain qualities, or a maximum size, in certain parts or organs of particular plants. Now, this object can be attained only by the application of those substances which we know to be indispensable to the development of these parts or organs, or by supplying the conditions necessary to the production of the qualities desired.

The rules of a rational system of agriculture should enable us, therefore, to give to each plant that which it requires for the attainment of the object in view.

The special object of agriculture is to obtain an abnormal development and production of certain parts of plants, or of certain vegetable matters, which are employed as food for man and animals, or for the purpose of industry.

The means employed for effecting these two purposes are very different. - Thus the mode of culture; employed for the purpose of procuring fine pliable straw for Florentine hats, is the very opposite to that which must be adopted in order to produce a maximum of corn from the same plant. Peculiar. methods must be used for the production of nitrogen in the seeds, others for giving strength and solidity to the straw, and others again must be followed when we wish to give such strength and solidity to 
the straw as will enable it to bear the weight of the ears.

We must proceed in the culture of plants in precisely the same manner as we do in the fattening of animals. The flesh of the stag and roe, or of wild animals in general, is quite devoid of fat, like the muscular flesh of the Arab; or it contains only small quantities of it. The production of flesh and fat may be artificially increased; all domestic animals, for example, contain much fat. We give food to animals, which increases the activity of certain organs, and is itself capable of being transformed into fat. We add to the quantity of food or we lessen the processes of respiration and perspiration by preventing motion. The conditions necessary to effect this purpose in birds are different from those in quadrupeds; and it is well known that charcoal powder produces such an excessive growth of the liver of a goose, as at length causes the death of the animal.

The increase or diminution of the vital activity of vegetables depends only on heat and solar light, which we have not arbitrarily at our disposal: all that we can do is to supply those substances which are adapted for assimilation by the power already present in the organs of the plant. But what then are these substances? They may easily be detected by the examination of a soil, which is always fertile in given cosmical and atmospheric conditions; for it is evident, that the knowledge of its state and composition must enable us to discover the circumstances under which a sterile soil may be rendered fertile. It is the duty of the chemist to explain the composition of a fertile- soil, but the discovery of its proper state or condition belongs to the agriculturist; our present business lies only with the former.

Arable land is originally formed by the crumbling of rocks, and its properties depend on the nature of their principal component parts. Sand, clay, and 
lime, are the names given to the principal constituents of the different kinds of soil.

Pure sand and pure limestones, in which there are no other inorganic substances except siliceous earth, carbonate or silicate of lime, form absolutely barren soils. But argillaceous earths form always a part of fertile soils. Now from whence come the argillaceous earths in arable land, what are their constituents, and what part do they play in favoring vegetation? They are produced by the disintegration of aluminous minerals by the action of the weather; the common potash and soda felspars, Labrador spar, mica, and the zeolites, are the most common aluminous earths, which undergo this change. These minerals are found mixed with other subtances in granite, gneiss, mica-slate, porphyry, clayslate, grauwacke, and the volcanic rocks, basalt, clinkstone, and lava. In the grauwacke, we have pure quartz, clay-slate, and lime; in the sandstones, quartz and loam. The transition limestone and the dolomites contain an intermixture of clay, felspar, porphyry, and clay-slate; and the mountain limestone is remarkable for the quantity of argillaceous earths which it contains. Jura limestone contains $3-20$, that of the Wurtemberg Alps $45-50$ per cent. of these earths. And in the muschelkalk and the calcaire grossier they exist in greater or less quantity.

It is known, that the aluminous, minerals are the most widely diffused on the surface of the earth, and as we have already mentioned, all fertile soils, or soils capable of culture, contain alumina as an invariable constituent. There must, therefore, be something in aluminous earth which enables it to exercise an influence on the life of plants, and to assist in their development. The property on which this depends is that of its invariably containing potash and soda.

Alumina exercises only an indirect influence on vegetation, by its power of attracting and retaining water and ammonia; it is itself very rarely found in 
the straw as will enable it to bear the weight of the ears.

We must proceed in the culture of plants in precisely the same manner as we do in the fattening of animals. The flesh of the stag and roe, or of wild animals in general, is quite devoid of fat, like the muscular flesh of the Arab; or it contains only small quantities of it. The production of flesh and fat may be artificially increased; all domestic animals, for example, contain much fat. We give food to animals, which increases the activity of certain organs, and is itself capable of being transformed into fat. We add to the quantity of food or we lessen the processes of respiration and perspiration by preventing motion. The conditions necessary to effect this purpose in birds are different from those in quadrupeds; and it is well known that charcoal powder produces such an excessive growth of the liver of a goose, as at length causes the death of the animal.

The increase or diminution of the vital activity of vegetables depends only on heat and solar light, which we have not arbitrarily at our disposal: all that we can do is to supply those substances which are adapted for assimilation by the power already present in the organs of the plant. But what then are these substances? They may easily be detected by the examination of a soil, which is always fertile in given cosmical and atmospheric conditions; for it is evident, that the knowledge of its state and composition must enable us to discover the circumstances under which a sterile soil may be rendered fertile. It is the duty of the chemist to explain the composition of a fertile-soil, but the discovery of its proper state or condition belongs to the agriculturist; our present business lies only with the former.

Arable land is originally formed by the crumbling of rocks, and its properties depend on the nature of their principal component parts. Sand, clay, and 
lime, are the names given to the principal constituents of the different kinds of soil.

Pure sand and pure limestones, in which there are no other inorganic substances except siliceous earth, carbonate or silicate of lime, form absolutely barren soils. But argillaceous earths form always a part of fertile soils. Now from whence come the argillaceous earths in arable land, what are their constituents, and what part do they play in favoring vegetation? They are produced by the disintegration of aluminous minerals by the action of the weather; the common potash and soda felspars, Labrador spar, mica, and the zeolites, are the most common aluminous earths, which undergo this change. These minerals are found mixed with other subtances in granite, gneiss, mica-slate, porphyry, clayslate, grauwacke, and the volcanic rocks, basalt, clinkstone, and lava. In the grauwacke, we have pure quartz, clay-slate, and lime; in the sandstones, quartz and loam. The transition limestone and the dolomites contain an intermixture of clay, felspar, porphyry, and clay-slate; and the mountain limestone is remarkable for the quantity of argillaceous earths which it contains. Jura limestone contains $3-20$, that of the Wurtemberg Alps $45-50$ per cent. of these earths. And in the muschelkalk and the calcaire grossier they exist in greater or less quantity.

It is known, that the aluminous, minerals are the most widely diffused on the surface of the earth, and as we have already mentioned, all fertile soils, or soils capable of culture, contain alumina as an invariable constituent. There must, therefore, be something in aluminous earth which enables it to exercise an influence on the life of plants, and to assist in their development. The property on which this depends is that of its invariably containing potash and soda.

Alumina exercises only an indirect influence on vegetation, by its power of attracting and retaining water and ammonia; it is itself very rarely found in 
kinds of plants grow with the greatest luxuriance. This fertility is owing to the alkalies which are contained in the lava, and which by exposure to the weather are rendered capable of being absorbed by plants. Thousands of years have been necessary to convert stones and rocks into the soil of arable land, and thousands of years more will be requisite for their perfect reduction, that is, for the complete exhaustion of their alkalies.

We see from the composition of the water in rivers, streamlets, and springs, how little rain-water is able to extract alkali from a soil, even after a term of years; this water is generally soft, and the common salt, which even the softest invariably contains, proves, that those alkaline salts, which are carried to the sea by rivers and streams, are returned again to the land by wind and rain.

Nature itself shows us what plants require at the commencement of the development of their germs and first radicle fibres. Becquerel has shown, that the graminea, leguminosa, crucifer $a$, cichoracea, umbelliferce, conifera, and cucurbitacea emit acetic acid during germination. A plant which has just broken through the soil, and a leaf just burst open from the bud, furnish ashes by incineration, which contain as much, and generally more, of alkaline salts than at any period of their life. (De Saussure.) Now we know also, from the experiments of Becquerel, in what manner these alkaline salts enter young plants; the acetic acid formed during germination is diffused through the wet or moist soil, becomes saturated with lime, magnesia, and alkalies, and is again absorbed by the radicle fibres in the form of neutral salts. After the cessation of life, when plants are subjected to decomposition by means of decay and putrefaction, the soil receives again that which had been extracted from it.

Let us suppose, that a soil has been formed by the action of the weather on the component parts of granite, grauwacke, mountain limestone, or porphy- 
ry, and that nothing has vegetated on it for thousands of years. Now this soil would become a magazine of alkalies in a condition favorable for their assimilation by the roots of plants.

The interesting experiments of Struve have proved that water impregnated with carbonic acid decomposes rocks which contain alkalies, and then dissolves a part of the alkaline carbonates. It is evident that plants also, by producing carbonic acid during their decay, and by means of the acids which exude from their roots in the living state, contribute no less powerfully to destroy the coherence of rocks. Next to the action of air, water, and change of temperature, plants themselves are the most powerful agents in effecting the disintegration of rocks.

Air, water, and the change of temperature prepare the different species of rocks for yielding to plants the alkalies which they contain. A soil which has been exposed for centuries to all the influences which affect the disintegration of rocks, but from which the alkalies have not been removed, will be able to afford the means of nourishment to those vegetables which require alkalies for their growth during many years; but it must gradually become exhausted, unless those alkalies which have been removed are again replaced; a period, therefore, will arrive when it will be necessary to expose it from time to time to a further disintegration, in order to obtain a new supply of soluble alkalies. For small as is the quantity of alkali which plants require, it is nevertheless quite indispensable for their perfect development. But when one or more years have elapsed without any alkalies having been extracted from the soil, a new harvest may be expected.

The first colonists of Virginia found a country the soil of which was similar to that mentioned above; harvests of wheat and tobacco were obtained for a century from one and the same field, without the aid of manure; but now whole districts are converted into unfruitful pasture-land, which without manure 
produces neither wheat nor tobacco. From every acre of this land there were removed in the space of one hundred years 13,200 lbs. of alkalies in leaves, grain, and straw ; it became unfruitful, therefore, because it was deprived of every particle of alkali, which had been reduced to a soluble state, and because that which was rendered soluble again in the space of one year was not sufficient to satisfy the demands of the plants. Almost all the cultivated land in Europe is in this condition; fallow is the term applied to land left at rest for further disintegration. It is the greatest possible mistake to suppose that the temporary diminution of fertility in a soil is owing to the loss of humus; it is the mere consequence of the exhaustion of the alkalies.

Let us consider the condition of the country around Naples, which is famed for its fruitful cornland; the farms and villages are situated from eighteen to twenty-four miles distant from one another, and between them there are no roads, and consequently no transportation of manure. Now corn has been cultivated on this land for thousands of years, without any part of that which is annually removed from the soil being artificially restored to it. How can any influence be ascribed to humus under such circumstances, when it is not even known whether humus was ever contained in the soil?

The method of culture in that district completely explains the permanent fertility. It appears very bad in the eyes of our agriculturists, but there it.is the best plan which could be adopted. A field is cultivated once every three years and is in the intervals allowed to serve as a sparing pasture for cattle. The soil experiences no change in the two years during which it there lies fallow, further than that it is exposed to the influence of the weather, by which a fresh portion of the alkalies contained in it are again set free or rendered soluble. The animals fed on these fields yield nothing to these soils which they did not formerly possess. The 
weeds upon which they live spring from the soil, and that which they return to it as excrement must always be less than that which they extract. The fields, therefore, can have gained nothing from the mere feeding of cattle upon them; on the contrary, the soil must have lost some of its constituents.

Experience has shown in agriculture that wheat should not be cultivated after wheat on the same soil, for it belongs with tobacco to the plants which exhaust a soil. But if the humus of a soil gives it the power of producing corn, how happens it that wheat does not thrive in many parts of Brazil, where the soils are particularly rich in this substance, or in our own climate, in soils formed of mouldered wood; that its stalk under these circumstances attains no strength, and droops prematurely? The cause is this, that the strength of the stalk is due to silicate of potash, and that the corn requires phosphate of magnesia, neither of which substances a soil of humus can afford, since it does not contain them; the plant may, indeed, under such circumstances, become an herb, but will not bear fruit.

Again, how does it happen that wheat does not flourish on a sandy soil, and that a calcareous soil is also unsuitable for its growth, unless it be mixed with a considerable quantity of clay?* It is because these soils do not contain alkalies in sufficient quantity, the growth of wheat being arrested by this circumstance, even should all other substances be presented in abundance.

It is not mere accident that only trees of the fir tribe grow on the sandstone and limestone of the Carpathian mountains and the Jura, whilst we find

* In consequence of these remarks in the former edition of this work, Professor Wöhler of Gottingen has made several accurate analyses of different kinds of limestone belonging to the secondary and tertiary formations. He obtained the remarkable result, that all those limestones, by the disintegration of which soils adapted for the culture of wheat - are formed, invariably contain a certain quantity of potash. The same observation has also recently been made by M. Kuhlmann of Lille. The latter observed that the efflorescence on the mortar of walls consists of the carbonates of soda and potash. $-L$. 
on soils of gneiss, mica-slate, and granite in Bavaria, of clinkstone on the Rhone, of basalt in Vogelsberge, and of clay-slate on the Rhine and Eifel, the finest forests of other trees, which cannot be produced on the sandy or calcareous soils upon which pines thrive. It is explained by the fact that trees, the leaves of which are renewed annually, require for their leaves six or ten times more alkalies than the fir-tree or pine, and hence when they are placed in soils in which alkalies are contained in very small quantity, do not attain maturity.* When we see such trees growing on a sandy or calcareous soil the red-beech, the service-tree, and the wild-cherry for example, thriving luxuriantly on limestone, we may be assured that alkalies are present in the soil, for they are necessary to their existence. Can we, then, regard it as remarkable that such trees should thrive in America, on those spots on which forests of pines which have grown and collected alkalies for centuries, have been burnt, and to which the alkalies are thus at once restored; or that the Spartium scoparium;Erysimum latifolium, Blitum capitatum, Senecio viscosus, plants remarkable for the quantity of alkalies contained in their ashes, should grow with the greatest luxuriance on the localities of conflagrations? $†$.

Wheat will not grow on a soil which has produced wormwood, and, vice versâ, wormwood does not thrive where wheat has grown, because they are mutually prejudicial by appropriating the alkalies of the soil.

One hundred parts of the stalks of wheat yield

* One thousand parts of the dry leaves of oaks yielded 55 parts of ashes, of which 24 parts consisted of alkalies soluble in water; the same quantity of pine-leaves gave only 29 parts of ashes, which contain 4.6 parts of soluble salts. (De Saussure.)

$\dagger$ After the great fire in London, large quantities of the Erysimum latifolium were observed growing on the spots where a fire had taken place. On a similar occasion the Blitum capitatum was seen at Copenhagen, the Senecio viscosus in Nassau, and the Spartium scoparium in Languedoc. After the burnings of forests of pines in North America, poplars grew on the same soil. - $L$. 
15.5 parts of ashes (H. Davy); the same quantity of the dry stalks of barley, 8.54 parts (Schrader); and one hundred parts of the stalks of oats, only 4.42 ; - the ashes of all these are of the same composition.

We have in these facts a clear proof of what plants require for their growth. Upon the same field, which will yield only one harvest of wheat, two crops of barley and three of oats may be raised.

All plants of the grass kind require silicate of potash. Now this is conveyed to the soil, or rendered soluble in it, by the irrigation of meadows. The equisetacea, the reeds and species of cane, for example, which contain such large quantities of siliceous earth, or silicate of potash, thrive luxuriantly in marshes, in argillaceous soils, and in ditches, streamlets, and other places where the change of water renews constantly the supply of dissolved silica. The amount of silicate of potash removed from a meadow in the form of hay is very considerable. We need only call to mind the melted vitreous mass found on a meadow between Manheim and Heidelberg after a thunder-storm. This mass was at first supposed to be a meteor, but was found on examination (by Gmelin) to consist of silicate of potash; a flash of lightning had struck a stack of hay, and nothing was found in its place except the melted ashes of the hay.

Potash is not the only substance necessary for the existence of most plants; indeed it has been already shown that the potash may be replaced in many cases by soda, magnesia, or lime; but other substances besides alkalies are required to sustain the life of plants.

Phosphoric acid has been found in the ashes of all plants hitherto examined, and always in combination with alkalies or alkaline earths.* Most seeds con-

* Professor Connall was lately kind enough to show me about half an ounce of a saline powder, which had been taken from an interstice in the body of a piece of teak timber. It consisted essentially of phos- 
tain certain quantities of phosphates. In the seeds of different kinds of corn particularly, there is abundance of phosphate of magnesia.

Plants obtain their phosphoric acid from the soil. It is a constituent of all land capable of cultivation, and even the heath at Lüneburg contains it in appreciable quantity. Phosphoric acid has been detected also in all mineral waters in which its presence has been tested; and in those in which it has not been found it has not been sought for. The most superficial strata of the deposits of sulphuret of lead (galena) contain crystallized phosphate of lead (greenlead ore); clay-slate, which forms extensive strata, is covered in many places with crystals of phosphate of alumina (Wavellite); all its fractured surfaces are overlaid with it. Phosphate of lime (Apatite) is found even in the volcanic boulders on the Laacher See in the Eifel, near Andernach.*

The soil in which plants grow furnishes them with phosphoric acid, and they in turn yield it to animals, to be used in the formation of their bones, and of those constituents of the brain which contain phosphorus. Much more phosphorus is thus afforded to the body than it requires, when flesh, bread, fruit, and husks of grain are used for food, and this excess is eliminated in the urine and the solid excrements. We may form an idea of the quantity of phosphate of magnesia contained in grain, when we consider that the concretions in the cæcum of horses

phate of lime, with small quantities of carbonate of lime and phosphate of magnesia. This powder had been sent to Sir David Brewster from India, with the assurance that it was the same substance which usually is found in the hollows of teak timber. It has long been known that silica, in the form of tabasheer, is secreted by the bamboo; but I am not aware that phosphates have been found in the same condition. Without more precise information, we must therefore suppose that they are left in the hollows by the decay of the wood. Decay is a slow process of combustion, and the incombustible ashes must remain after the organic matter has been consumed. But if this explanation be correct, the wood of the teak-tree must contain an enormous quantity of earthy phosphates. - ED.

* See the analyses of soils in the Appendix. 
consist of phosphate of magnesia and ammonia, which must have been obtained from the hay and oats consumed as food. Twenty-nine of these stones were taken after death from the rectum of a horse belonging to a miller, in Eberstadt, the total weight of which amounted to $3.3 \mathrm{lbs}$.; and Dr. F. Simon has lately described a similar concretion found in the horse of a carrier, which weighed $1.6 \mathrm{lb}$.

It is evident that the seeds of corn could not be formed without phosphate of magnesia, which is one of their invariable constituents; the plant could not under such circumstances reach maturity.

Some plants, however, extract other matters from the soil besides silica, potash, and phosphoric acid, which are essential constituents of the plants ordinarily cultivated.*. These other matters, we must suppose, supply, in part at least, the place and perform the functions of the substances just named. We may thus regard common salt, sulphate of potash, nitre, chloride of potassium, and other matters, as necessary constituents of several plants.

Clay-slate contains generally small quantities of oxide of copper; and soils formed from micaceous schist contain some metallic fluorides. Now, small quantities of these substances also are absorbed into plants, although we cannot affirm that they are necessary to them.

It appears that in certain cases fluoride of calcium $\dagger$ may take the place of the phosphate of lime in the bones and teeth; $\ddagger$ at least it is impossible otherwise to explain its constant presence in the bones of

* For more minute information regarding soils see the supplementary chapter at the end of Part I.

+ Fluorine is the base of the acid contained in Fluor or Derbyshire spar; with hydrogen it forms the hydrofluoric acid. The acid is separated by heating fluor spar with sulphuric acid, and is distinguished by its power of corroding glass, and of uniting with its silica. Compounds of Fluorine are called Fluorides, of the acid Hydrofluates. Calcium is the metallic base of lime.

$\$$ The earthy parts of bones are composed principally of the phosphate and carbonate of lime in various proportions, variable in different animals, and mixed with small quantities, equally variable, of phos- 
antediluvian animals, by which they are distinguished from those of a later period. The bones of human skulls found at Pompeii contain as much fluoric acid as those of animals of a former world, for if they be placed in a state of powder in glass vessels, and digested with sulphuric acid, the interior of the vessel will, after twenty-four hours, be found powerfully corroded (Liebig); whilst the bones and teeth of animals of the present day contain only traces of it. (Berzelius.)

De Saussure remarked, that plants require unequal quantities of the component parts of soils in different stages of their development; an observation of much importance in considering the growth of plants. Thus, wheat yielded ${ }_{1000}^{79}$ of ashes a month before blossoming, ${ }_{1000}^{54}$ while in blossom, and $\frac{33}{1000}$ after the ripening of the seeds. It is therefore evident, that

phate of magnesia and fluate of lime. By acting upon calcined bones with sulphuric acid fluoric acid is disengaged. The following analyses of the hones of man and horned cattle, are given by Berzelius.

\section{Human bone. Ox bone.}

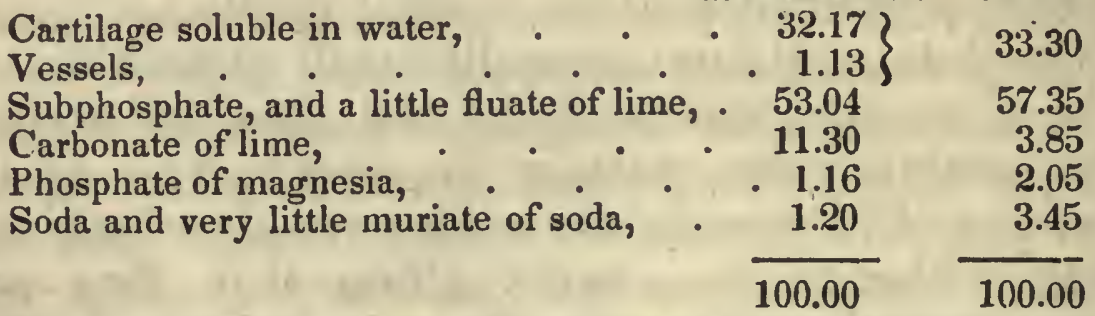

The bones of man contain three times as much carbonate of lime as those of the $0 x$, and the latter are richer in phosphate of lime and magnesia in the same proportion.

The following are the relative proportions of phosphate and carbonate of lime in bones of different animals, according to De Barros.

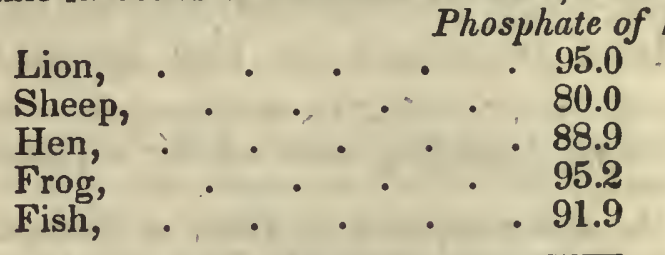

Carbonate of Lime.

2.5

The enamel of the teeth is composed of

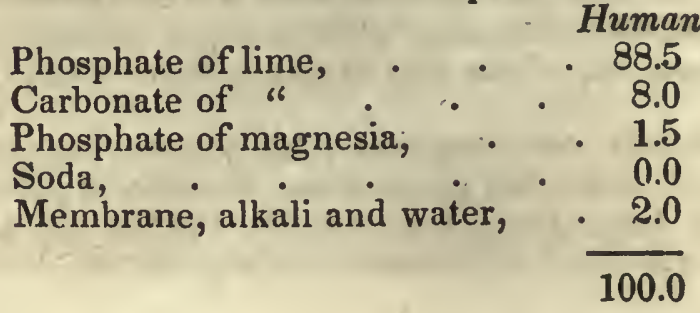


wheat, from the time of its flowering, restores a part of its organic constituents to the soil, although the phosphate of magnesia remains in the seeds.

The fallow-time, as we have already shown, is that period of culture during which land is exposed to a progressive disintegration by means of the influence of the atmosphere, for the purpose of rendering a certain quantity of alkalies capable of being appropriated by plants.

Now, it is evident, that the careful tilling of fallow-land must increase and accelerate this disintegration. For the purpose of agriculture, it is quite indifferent, whether the land is covered with weeds, or with a plant which does not abstract the potash inclosed in it. Now many plants in the family of the leguminosa are remarkable on account of the small quantity of alkalies or salts in general which they contain; the Windsor bean (Vicia Faba), for example, contains no free alkalies, and not one per cent. of the phosphates of lime and magnesia. (Einhof.) The bean of the kidney-bean (Phaseolus vulgaris ) contains only traces of salts. (Braconnot.) The stem of Lucern (Medicago sativa) contains only 0.83 per cent., that of the Lentil (Ervum Lens) only 0.57 of phosphate of lime with albumen. (Crome.) Buck-wheat dried in the sun yields only 0.681 per cent. of ashes, of which 0.09 parts are soluble salts. (Zenneck.)* These plants belong to

* The small quantity of phosphates which the seeds of the lentils, beans, and peas contain, must be the cause of their small value as articles of nourishment, since they surpass all other vegetable food in the quantity of nitrogen which enters into their composition. But as the component parts of the bones (phosphate of lime and magnesia) are absent, they satisfy the appetite without increasing the strength. The following is an analysis of lentils (Playfair). 6.092 grammes lost 0.972 grammes of water at $212^{\circ}$. 0.566 grammes, burned with oxide of copper, gave 0.910 grammes carbonic acid and 0336 grammes of water. The lentils on combustion with oxide of copper, yielded a gas, in which the proportion of the nitrogen to the carbonic acid was as $1: 16$.

Carbon $\quad 44 \cdot 45$

Hydrogen $6 \cdot 59$

Nitrogen 6.42

Water

15.95 
those which are termed fallow-crops, and the cause wherefore they do not exercise any injurious influence on corn which is cultivated immediately after them is, that they do not extract the alkalies of the soil, and only a very small quantity of phosphates.

It is evident that two plants growing beside each other will mutually injure one another, if they withdraw the same food from the soil. Hence it is not surprising that the wild chamomile (Matricaria Chamomilla) and Scotch broom (Spartium Scoparium) impede the growth of corn, when it is considered that both yield from 7 to $\mathbf{7 \cdot 4 3}$ per cent. of ashes, which contain $\frac{6}{10}$ of carbonate of potash. The darnel and the fleabane (Erigeron acre) blossom and bear fruit at the same time as corn, so that when growing mingled with it, they will partake of the component parts of the soil, and in proportion to the vigor of their growth, that of the corn must decrease; for what one receives, the others are deprived of. Plants will, on the contrary, thrive beside each other, either when the substances necessary for their growth which they extract from the soil are of different kinds, or when they themselves are not both in the same stages of development at the same time.

On a soil, for example, which contains potash, both wheat and tobacco may be reared in succession, because the latter plant does not require phosphates, salts which are invariably present in wheat, but requires only alkalies, and food containing nitrogen.

According to the analysis of Posselt and Reimann, 10,000 parts of the leaves of the tobacco-plant contain 16 parts of phosphate of lime, 8.8 parts of silica, and no magnesia; whilst an equal quantity of wheat straw contains $47 \cdot 3$ parts, and the same quantity of the grain of wheat 99.45 parts of phosphates. (De Saussure.)

Now, if we suppose that the grain of wheat is equal to half the weight of its straw, then the quantity of phosphates extracted from a soil by the same 
weights of wheat and tobacco must be as 97.7: 16 . This difference is very considerable. The roots of tobacco, as well as those of wheat, extract the phosphates contained in the soil, but they restore them again, because they are not essentially necessary to the development of the plant.

\section{CHAPTER VIII.}

ON THE ALTERNATION OF CROPS.

IT has long since been found by experience, that the growth of annual plants is rendered imperfect, and their crops of fruit or herbs less abundant, by cultivating them in successive years on the same soil, and that, in spite of the loss of time, a greater quantity of grain is obtained when a field is allowed to lie uncultivated for a year. During this interval of rest, the soil, in a great measure, regains its original fertility.

It has been further observed, that certain plants, such as peas, clover, and flax, thrive on the same soil only after a lapse of years; whilst others, such as hemp, tobacco, helianthus tuberosus, rye, and oats, may be cultivated in close succession when proper manure is used. It has also been found, that several of these plants improve the soil, whilst others, and these are the most numerous, impoverish or exhaust it. Fallow turnips, cabbage, beet, spelt, summer and winter barley, rye and oats, are considered to belong to the class which impoverish a soil; whilst by wheat, hops, madder, late turnips, hemp, poppies, teasel, flax, weld, and licorice, it is supposed to be entirely exhausted.

The excrements of man and animals have been employed from the earliest times for the purpose of increasing the fertility of soils; and it is completely established by all experience, that they restore cer- 
tain constituents to the soil, which are removed with the roots, fruit, or grain, or entire plants grown upon it.

But it has been observed, that the crops are not always abundant in proportion to the quantity of manure employed, even although it may have been of the most powerful kind; that the produce of many plants, for example, diminishes, in spite of the apparent replacement by manure of the substances removed from the soil, when they are cultivated on the same field for several years in succession.

On the other hand it has been remarked, that a field which has become unfitted for a certain kind of plants was not on that account unsuited for another; and upon this observation, a system of agriculture has been gradually founded, the principal object of which is to obtain the greatest possible produce with the least expense of manure.

Now it was deduced from all the foregoing facts, that plants require for their growth different constituents of soil, and it was very soon perceived, that an alternation of the plants cultivated maintained the fertility of a soil quite as well as leaving it at rest or fallow. It was evident, that all plants must give back to the soil in which they grow different proportions of certain substances, which are capable of being used as food by a succeeding generation.

But agriculture has hitherto never sought aid from chemical principles, based on the knowledge of those substances which plants extract from the soil on which they grow, and of those restored to the soil by means of manure. The discovery of such principles will be the task of a future generation, for what can be expected from the present, which recoils with seeming distrust and aversion from all the means of assistance offered it by chemistry, and which does not understand the art of making a rational application of chemical discoveries? A future generation, however, will derive incalculable advantage from these means of help. 
Of all the views which have been adopted regarding the cause of the favorable effects of the alternations of crops, that proposed by M. Decandolle alone deserves to be mentioned as resting on a firm basis.

Decandolle supposes, that the roots of plants imbibe soluble matter of every kind from the soil, and thus necessarily absorb a number of substances which are not adapted to the purposes of nutrition, and must subsequently be expelled by the roots, and returned to the soil as excrements. Now as excrements cannot be assimilated by the plant which ejected them, the more of these matters which the soil contains, the more unfertile must it be for the plants of the same species. These excrementitious matters may, however, still be capable of assimilation by another kind of plants, which would thus remove them from the soil, and render it again fertile for the first. And if the plants last grown also expel substances from their roots, which can be appropriated as food by the former, they will improve the soil in two ways.

Now a great number of facts appear at first sight to give a high degree of probability to this view. Every gardener knows, that a fruit-tree cannot be made to grow on the same spot where another of the same species has stood; at least not until after a lapse of several years. Before new vine-stocks are planted in a vineyard from which the old have been rooted out, other plants are cultivated on the soil for several years. In connexion with this it has been observed, that several plants thrive best when growing beside one another; and, on the contrary, that others mutually prevent each other's development. Whence it was concluded, that the beneficial influence in the former case depended on a mutual interchange of nutriment between the plants, and the injurious one in the latter on a poisonous action of the excrements of each on the other respectively.* 
A series of experiments by Macaire-Princep gave great weight to this theory. He proved beyond all doubt, that many plants are capable of emitting extractive matter from their roots. He found that the excretions were greater during the night than by day (?), and that the water in which plants of the family of the Leguminosa grew acquired a brown color. Plants of the same species placed in water impregnated with these excrements were impeded in their growth, and faded prematurely, whilst, on the contrary, corn-plants grew vigorously in it, and the color of the water diminished sensibly; so that it appeared as if a certain quantity of the excrements of the Leguminose had really been absorbed by the corn-plants. These experiments afforded, as their main result, that the characters and properties of the excrements of different species of plants are different from one another, and that some plants expel excrementitious matter of an acid and resinous character; others mild substances resembling gum. The former of these, according to Macaire-Princep, may be regarded as poisonous, the latter as nutritious.

The experiments of Macaire-Princep afford positive proof that the roots, probably of all plants, expel matters, which cannot be converted in their organism either into woody fibre, starch, vegetable albumen, or gluten, since their expulsion indicates that they are quite unfitted for this purpose. But they

ous to plants of similar species, has been inferred from the fact, that a soil, in which peach or apple trees have grown, is unfit for young shoots of the same description, so as to render it a necessary rule in practice, that a piece of ground should be occupied by forest and by fruit trees alternately.

Reference has also been made to a circumstance, which most travellers in the United States have remarked, and which I myself, during my tour in that country, had frequent opportunities of substantiating, namely, that where a forest of oak or of maple has been destroyed, the trees, that are apt to shoot up spontaneously in their place, are of the fir-tribe; whereas, if a pine forest be cut down, young oaks and other allied species will make their appearance afterwards. - DauBENy's Lectures on Agriculture.

For an account of experiments on this subject now in progress at $O x-$ ford, see Appendix. 
cannot be considered as a confirmation of the theory of Decandolle, for they leave it quite undecided whether the substances were extracted from the soil, or formed by the plant itself from food received from another source. It is certain, that the gummy and resinous excrements observed by Macaire-Princep could not have been contained in the soil, and as we know that the carbon of a soil is not diminished by culture, but, on the contrary, increased, we must conclude that all excrements which contain carbon must be formed from the food obtained by plants from the atmosphere. Now, these excrements are compounds, produced in consequence of the transformations of the food, and of the new forms which it assumes by entering into the composition of the various organs.

M. Decandolle's theory is properly a modification of an earlier hypothesis, which supposed that the roots of different plants extracted different nutritive substances from the soil, each plant selecting that which was exactly suited for its assimilation. According to this hypothesis, the matters incapable of assimilation are not extracted from the soil, whilst M. Decandolle considers that they are returned to it in the form of excrements. Both views explain how it happens that after corn, corn cannot be raised with advantage, nor after peas, peas; but they do not explain how a field is improved by lying fallow, and this in proportion to the care with which it is tilled and kept free from weeds; nor do they show how a soil gains carbonaceous matter by the cultivation of certain plants such as lucern and sainfoin.

Theoretical considerations on the process of nutrition, as well as the experience of all agriculturists, so beautifully illustrated by the experiments of Macaire-Princep, leave no doubt that substances are excreted from the roots of plants, and that these matters form the means by which the carbon received from humus in the early period of their growth is restored to the soil. But we may now inquire wheth- 
er these excrements, in the state in which they are expelled, are capable of being employed as food by other plants.

The excrements of a carnivorous animal contain no constituents fitted for the nourishment of another of the same species; but it is possible that an herbivorous animal, a fish, or a fowl, might find in them undigested matters capable of being digested in their organism, from the very circumstance of their organs of digestion having a different structure. This is the only sense in which we can conceive that the excrements of one animal could yield matter adapted for the nutrition of another.

A number of substances contained in the food of animals pass through their alimentary organs without change, and are expelled from the system; these are excrements but not excretions. Now a part of such excrementitious matter might be assimilated in passing through the digestive apparatus of another animal. The organs of secretion form combinations of which only the elements were. contained in the food. The production of these new compounds is a consequence of the changes which the food undergoes in becoming chyle and chyme, and of the further transformations to which these are subjected by entering into the composition of the organism. These matters, likewise, are eliminated in the excrements, which must therefore consist of two different kinds of substances, namely, of the indigestible constituents of the food, and of the new compounds formed by the vital process. The latter substances have been produced in consequence of the formation of fat, muscular fibre, cerebral and nervous substance, and are quite incapable of being converted into the same substances in any other animal organism.

Exactly similar conditions must subsist in the vital processes of plants. When substances which are incapable of being employed in the nutrition of a plant exist in the matter absorbed by its roots, they must be again returned to the soil. Such excrements 
might be serviceable and even indispensable to the existence of several other plants. But substances that are formed in a vegetable organism during the process of nutrition, which are produced, therefore, in consequence of the formation of woody fibre, starch, albumen, gum, acids, \&c., cannot again serve in any other plants to form the same constituents of vegetables.

The consideration of these facts enables us to distinguish the difference between the views of Decandolle and those of Macaire-Princep. The substances which the former physiologist viewed as excrements, belonged to the soil; they were undigested matters, which although not adapted for the nutrition of one plant might yet be indispensable to another. Those matters, on the contrary, designated as excrements by Macaire-Princep, could only in one form serve for the nutrition of vegetables. It is scarcely necessary to remark, that this excrementitious matter must undergo a change before another season. During autumn and winter it begins to suffer a change from the influence of air and water; its putrefaction, and at length, by continued contact with the air, which tillage is the means of procuring, its decay are effected; and at the commencement of spring it has become converted, either in whole or in part, into a substance which supplies the place of humus, by being a constant source of carbonic acid.

The quickness with which this decay of the excrements of plants proceeds depends on the composition of the soil, and on its greater or less porosity. It will take place very quickly in a calcareous soil : for the power of organic excrements to attract oxygen and to putrefy is increased by contact with the alkaline constituents, and by the general porous nature of such kinds of soil, which freely permit the access of air. But it requires a longer time in heavy soils consisting of loam or clay.

The same plants can be cultivated with advantage on one soil after the second year, but in others not 
until the fifth or ninth, merely on account of the change and destruction of the excrements, which have an injurious influence on the plants being completed in the one, in the second year; in the others, not until the ninth.

In some neighborhoods clover will not thrive till the sixth year, in others not till the twelfth; flax in the second or third year. All this depends on the chemical nature of the soil, for it has been found by experience, that in those districts where the intervals at which the same plants can be cultivated with advantage are very long, the time cannot be shortened even by the use of the most powerful manures: The destruction of the peculiar excrements of one crop must have taken place before a new crop can be produced.

Flax, peas, clover, and even potatoes, are plants the excrements of which, in argillaceous soils, require the longest time for their conversion into humus; but it is evident, that the use of alkalies and burnt lime, or even small quantities of ashes which have not been lixiviated, must enable a soil to permit the cultivation of the same plants in a much shorter time.

A soil lying fallow owes its earlier fertility, in part, to the destruction or conversion into humus of the excrements contained in it, which is effected during the fallow season, at the same time that the land is exposed to a further disintegration.

In the soils in the neighborhood of the Rhine and Nile, which contain much potash, and where crops can be obtained in close succession from the same field, the fallowing of the land is superseded by the inundation; the irrigation of meadows effects the same purpose. It is because the water of rivers and streams contains oxygen in solution, that it effects the most complete and rapid putrefaction of the excrements contained in the soil which it penetrates, and in which it is continually renewed. If it was the water alone which produced this effect, marshy 
meadows should be most fertile. Hence it is not sufficient in irrigating meadows to convert them into marshes, by covering for several months their surface with water, which is not renewed; for the advantage of irrigation consists principally in supplying oxygen to the roots of plants. 'The quantity of water necessary for this purpose is very small, so that it is sufficient to cover the meadow with a very thin layer, if this be frequently renewed.

The cultivation of meadows forms one of the most important branches of rural economy. It contributes materially to the prosperity of the agriculturist by increasing his stock of cattle, and consequently by furnishing him with manure, which may be applied to the augmentation of his crops. Indeed, the great progress which has been made in Germany in the improvement of cattle is mainly attributable to the attention which is devoted in that country to the culture of meadows. The environs of Siegin, in Nassau, are particulary famed in this respect, and every year a large number of young farmers repair to it, for the purpose of studying this branch of agriculture in situ. In that district the culture of grass has attained such great perfection, that the produce of their meadow-land far exceeds that obtained in any other part of Germany. This is effected simply by preparing the ground in such a manner as to enable it to be irrigated both in spring and in autumn. The surface of the soil is fitted to suit the locality, and the quantity of water which can be commanded. Thus if the meadows be situated upon a declivity, banks of from one to two feet in height are raised at short distances from each other. The water is admitted by small channels upon the most elevated bank, and allowed to discharge itself over the sides in such a manner as to run upon the bank situated below. The grass grown upon meadows irrigated in this way is three or four times higher than that obtained from fields which are covered with: water that is deprived of all egress and renewal. 
It follows from what has preceded, that the advantage of the alternation of crops is owing to two causes.

A fertile soil ought to afford to a plant all the inorganic bodies indispensable for its existence in sufficient quantity and in such condition as allows their absorption.

All plants require alkalies, which are contained in some, in the Graminea for example, in the form of silicates; in others, in that of tartrates; citrates, acetates, or oxalates.

When these alkalies are in combination with silicic acid, the ashes obtained by the incineration of the plant contain no carbonic acid; but when they are united with organic acids, the addition of a mineral acid to their ashes causes an effervescence.

A third species of plants requires phosphate of lime, another phosphate of magnesia, and several do not thrive without carbonate of lime.

Silicic acid * is the first solid substance taken up by plants; it appears to be the material from which

* Silica, or siliceous earth, is the most abundant ingredient in the mineral kingdom, being one of the constituents of most rocks, and extensively distributed over the earth in the form of sand, quartz, carnelian, flint, \&c., \&c. It is also held in solution by the water of hot springs, as in the Geysers of Iceland, and the Azores, from which it is deposited, forming what is called siliceous sinter, and often incrusting the stems of plants and other bodies. The vegetable matter in some instances has entirely disappeared, and the silica having taken its place we have silicified or petrified wood, \&c. See Webster's Description of the Island of St. Michael, p. 208. From silica a substance is obtained which is considered as its base and called silicon and silicium. This base, combined with oxygen, constitutes silica, which is capable of combining with other bases; from this and other properties it is called silicic acid. By combination with other substances, as potash, soda, \&c., silica becomes soluble in water. These compounds are called silicates. A white, earthy substance is found beneath peat and in swampy lands and ponds, which has long been mistaken for calcareous marl. It has been proved to consist of the siliceous skeletons of "infusorial vegetables, if they may be so called, or of those equivocal beings, which occupy the borders of the two kingdoms, and render it difficult, not to say impossible, to draw the line between them." This siliceous deposite has been found under nearly every peat bog in this country which has been examined. See Professor Bailey's paper in American Journal of Science, Vol. XXXV. p. 118, and Vol. XL. p. 174. 
the formation of the wood takes its origin, acting like a grain of sand around which the first crystals form in a solution of a salt which is in the act of crystallizing. Silicic acid appears to perform the function of woody fibre in the Equisetacee and bamboos, just as the crystalline salt, oxalate of lime, does in many of the lichens.

When we grow in the same soil for several years in succession different plants, the first of which leaves behind that which the second, and the second that which the third may require, the soil will be a fruitful one for all the three kinds of produce. If the first plant, for example, be wheat, which consumes the greatest part of the silicate of potash in a . soil, whilst the plants which succeed it are of such a kind as require only small quantities of potash, as is the case with Leguminosa, turnips, potatoes, \&c., the wheat may be again sowed with advantage after the fourth year; for during the interval of three years the soil will, by the action of the atmosphere, be rendered capable of again yielding silicate of potash in sufficient quantity for the young plants.

The same precautions must be observed with regard to the other inorganic constituents, when it is desired to grow different plants in succession on the same soil: for a successive growth of plants which extract the same components paris, must gradually render it incapable of producing them. Each of these plants during its growth returns to the soil a certain quantity of substances containing carbon, which are gradually converted into humus, and are for the most part equivalent to as much carbon as the plants had formerly extracted from the soil in a state of carbonic acid. But although this is sufficient to bring many plants to maturity, it is not enough to furnish their different organs with the greatest possible supply of nourishment. Now the object of

* Silica is found in the joints of bamboos, in the form of small round globules, which have received the name of Trbasheer, and are distinguished by their remarkable optical properties. - Ev. 
agriculture is to produce either articles of commerce, or food for man and animals; but a maximum of produce in plants is always in proportion to the quantity of nutriment supplied to them in the first stage of their development.

The nutriment of young plants consists of carbonic acid, contained in the soil in the form of humus, and of nitrogen in the form of ammonia, both of which must be supplied to the plants, if the desired purpose is to be accomplished. The formation of ammonia cannot be effected on cultivated land, but humus may be artificially produced; and this must be considered as an important object in the alternation of crops, and as the second reason of its peculiar advantages.

The sowing of a field with fallow plants, such as clover, rye, buck-wheat, \&c., and the incorporation of the plants, when nearly at blossom, with the soil, affect this supply of humus in so far, that young plants subsequently growing in it find, at a certain period of their growth, a maximum of nutriment, that is, matter in the process of decay.

The same end is obtained, but with much greater certainty, when the field is planted with sainfoin or lucern.* These plants are remarkable on account of the great ramification of their roots, and strong development of their leaves, and for requiring only a small quantity of inorganic matter. Until they reach a certain period of their growth, they retain all the carbonic acid and ammonia which may have been conveyed to them by rain and the air, for that which is not absorbed by the soil is appropriated by the leaves; they also possess an extensive four or

* The alternation of crops with sainfoin and lucern is now universally adopted in Bingen and its vicinity, as well as in the Palatinate; the fields in these districts receive manure only once every nine years. In the first years after the land has been manured turnips are sown upon it, in the next following years barley, with sainfoin or lucern; in the seventh year potatoes, in the eighth wheat, in the ninth barley; on the tenth year it is manured, and then the same rotation again takes place. $-L$. 
six-fold surface, capable of assimilating these bodies, and of preventing the volatilization of the ammonia from the soil, by completely covering it in.

An immediate consequence of the production of the green principle of the leaves, and of their remaining component parts, as well as those of the stem, is the equally abundant excretion of organic matters into the soil from the roots.

The favorable influence which this exercises on the land, by furnishing it with matter capable of being converted into humus, lasts for several years, but barren spots gradually appear after the lapse of some time. Now it is evident that, after from six to seven years, the ground must become so impregnated with excrements, that every fibre of the root will be surrounded with them. As they remain for some time in a soluble condition, the plants must absorb part of them and suffer injurious effects in consequence, because they are not capable of assimilation. When such a field is observed for several years, it is seen that the barren spots are again covered with vegetation, (the same plants being always supposed to be grown,) whilst new spots become bare and apparently unfruitful, and so on alternately. The causes which produce this alternate barrenness and fertility in the different parts of the land are evident. The excrements upon the barren spots receiving no new addition, and being subjected to the influence of air and moisture, they pass into putrefaction, and their injurious influence ceases. The plants now find those substances which formerly prevented their growth removed, and in their place meet with humus, that is, vegetable matter in the act of decay.

We can scarcely suppose a better means of producing humus than by the growth of plants, the leaves of which are food for animals; for they prepare the soil for plants of every other kind, but particularly for those to which, as to rape and flax, 
the presence of humus is the most essential condition of growth.

The reasons why this interchange of crops is so advantageous, - the principles which regulate this part of agriculture, are, therefore, the artificial production of humus, and the cultivation of different kinds of plants upon the same field, in such an order of succession, that each shall extract only certain components of the soil, whilst it leaves behind or restores those which a second or third species of plant may require for its growth and perfect development.

Now, although the quantity of humus in a soil may be increased to a certain degree by an artificial cultivation, still, in spite of this, there cannot be the smallest doubt that a soil must gradually lose those of its constituents which are removed in the seeds, roots, and leaves of the plants raised upon it. The fertility of a soil cannot remain unimpaired, unless we replace in it all those substances of which it has been thus deprived.

Now this is effected by manure.

\section{CHAPTER IX.}

\section{OF MANURE.}

WHEN it is considered that every constituent of the body of man and animals is derived from plants, and that not a single element is generated by the vital principle, it is evident that all the inorganic constituents of the animal organism must be règarded, in some respect or other, as manure. During their life, the inorganic components of plants which are not required by the animal system, are disengaged from the organism, in the form of excrements. After their death, their nitrogen and carbon pass into the atmosphere as ammonia and carbonic acid, 
the products of their putrefaction, and at last nothing remains except the phosphate of lime and other salts in their bones. Now this earthy residue of the putrefaction of animals must be considered, in a rational system of agriculture, as a powerful manure for plants, because that which has been abstracted from a soil for a series of years must be restored to it, if the land is to be kept in a permanent condition of fertility.

\section{ANIMAL MANURES.}

We may now inquire whether the excrements of animals, which are employed as manure, are all of a like nature and power, and whether they, in every case, administer to the necessities of a plant by an identical mode of action. These points may easily be determined by ascertaining the composition of the animal excrements, because we shall thus learn what substances a soil really receives by their means. According to the common view, the action of solid animal excrements depends on the decaying organic matters which replace the humus, and on the presence of certain compounds of nitrogen, which are supposed to be assimilated by plants, and employed in the production of gluten and other azotized substances. But this view requires further confirmation with respect to the solid excrements of animals, for they contain so small a proportion of nitrogen, that they cannot possibly by means of it exercise any influence upon vegetation.

We may form a tolerably correct idea of the chemical nature of the animal excrement without further examination, by comparing the excrements of a dog with its food. When a dog is fed with flesh and bones, both of which consist in great part of organic substances containing nitrogen, a moist white excrement is produced which crumbles gradually to a dry powder in the air. 'This excrement consists of the 
phosphate of lime of the bones, and contains scarcely $\frac{1}{100}$ part of its weight of foreign organic substances. 'The whole process of nutrition in an animal consists in the progressive extraction of all the nitrogen from the food, so that the quantity of this element found in the excrements must always be less than that contained in the nutriment. The analysis of the excrements of a horse by Macaire and Marcet proves this fact completely. The portion of excrements subjected to analysis was collected whilst fresh, and dried in vacuo over sulphuric acid; 100 parts of it (corresponding to from 350 to 400 parts of the dung before being dried) contained 0.8 of nitrogen. Now every one who has had experience in this kind of analysis is aware, that a quantity under one per cent. cannot be determined with accuracy. We should, therefore, be estimating its proportion at a maximum, were we to consider it as equal to one-half per cent. It is certain, however, that these excrements are not entirely free from nitrogen, for they.emit ammonia. when digested with caustic potash.

The excrements of a cow, on combustion with oxide of copper, yielded a gas which contained one vol. of nitrogen gas, and $26.30 \mathrm{vol}$. of carbonic acid. 100 parts of fresh excrements contained

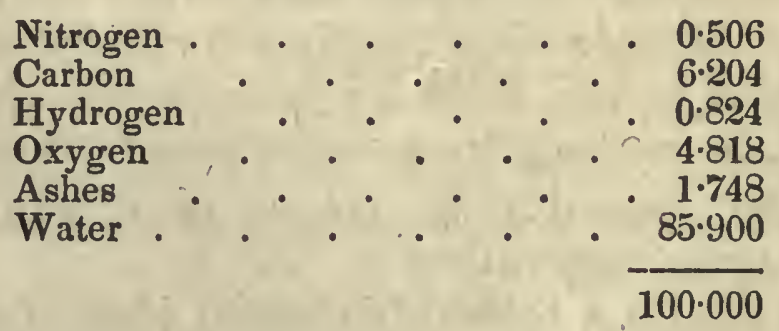

Now, according to the analysis of Boussingault, which merits the greatest confidence, hay contains one per cent. of nitrogen; consequently in the $25 \mathrm{lbs}$. of hay which a cow consumes daily, $\frac{1}{4}$ of a lb. of nitrogen must have been assimilated. This quantity of nitrogen entering into the composition of muscular fibre would yield $8.3 \mathrm{lbs}$. of flesh in its natural 
condition.* The daily increase in size of a cow is, however, much less than this quantity. We find that the nitrogen, apparently deficient, is actually contained in the milk and urine of the animal. The urine of a milch-cow contains less nitrogen than that of one which does not yield milk; and as long as a cow yields a plentiful supply of milk, it cannot be fattened. We must search for the nitrogen of the food assimilated, not in the solid, but in the liquid excrements. The influence which the former exercise on the growth of regetables does not depend upon the quantity of nitrogen which they contain. For if this were the case, hay should possess the same influence; that is, from 20 to $25 \mathrm{lbs}$. ought to have the same power as $100 \mathrm{lbs}$. of fresh cow-dung. But this is quite opposed to all experience.

Which then are the substances in the excrements of the cow and horse which exert an influence on vegetation?

When horse-dung is treated with water, a portion of it to the amount of 3 or $3 \frac{1}{2}$ per cent. is dissolved, and the water is colored yellow. The solution is found to contain phosphate of magnesia, and salts of soda, besides small quantities of organic matters. $\dagger$

* $100 \mathrm{lbs}$. of flesh contain on an average 15.86 of muscular fibre : 18 parts of nitrogen are contained in 100 parts of the latter. $-L$.

The flesh of animals when digested in repeated portions of cold water, affords albumen, saline substances, and coloring and extractive matters. When the part that is no longer acted on by cold water is digested in hot water, the cellular substance is removed in the form of gelatine, and fatty matter separates. The insoluble residue is principally fibrine.

The following is the proportion of water, albumen, and gelatine in the muscular parts of several animals and fishes.

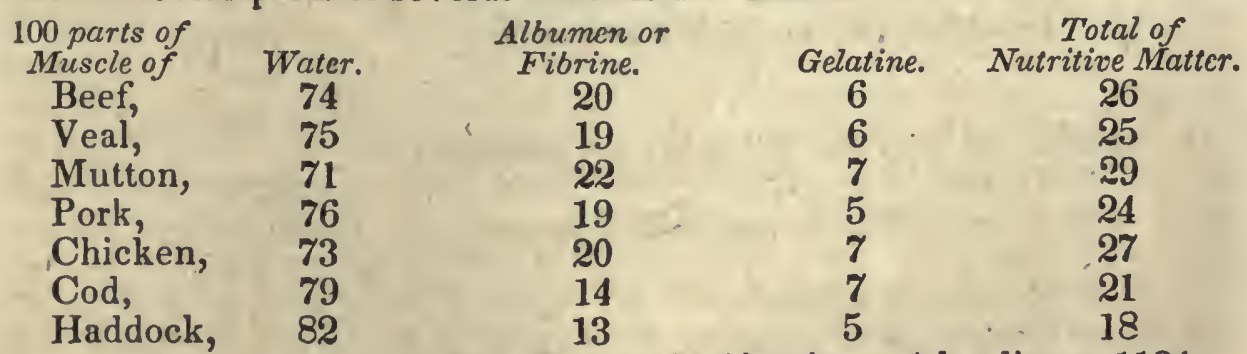

See BrANDE's Chemistry, 4th edit., p. 1184.

+ Dr. C. T. Jackson in his "Geological and Agricultural Survey of Rhode Island," (page 205,) gives the following analysis of horse-dung: 
The portion of the dung undissolved by the water yields to alcohol a resinous substance possessing all the characters of gall which has undergone some change; while the residue possesses the properties of sawdust, from which all soluble matter has been extracted by water, and burns without any smell. 100 parts of the fresh dung of a horse being dried at $100^{\circ} \mathrm{C}$. $\left(212^{\circ} \mathrm{F}\right.$.) leave from 25 to 30 or 31 parts of solid substances, and contained, accordingly, from 69 to 75 parts of water. From the dried excrements, we obtain, by incineration, variable quantities of salts and earthy matters according to the nature of the food which has been taken by the animal. Macaire and Marcet found 27 per cent. in the dung analyzed by them; I obtained only 10 per cent. from that of a horse fed with chopped straw, oats, and hay. It results then that with from 3900 to $4400 \mathrm{lbs}$. of fresh horse-dung, corresponding to $110 \mathrm{lbs}$. of dry dung, we place on the land from 2737 to $3006 \mathrm{lbs}$. of water, and from 804 to $992 \mathrm{lbs}$. of vegetable matter and altered gall, and also from 110 to $297 \mathrm{lbs}$. of salt and other inorganic substances.

The latter are evidently the substances to which our attention should be directed, for they are the same which formed the component parts of the hay, straw, and oats with which the horse was fed. Their

-500 grains, dried at a heat a little above that of boiling water, lost 357 grains of water. The dry mass weighing 143 grains was burned, and left 8 grains of ashes, of which 4.80 grains were soluble in dilute nitric acid, and 3.20 insoluble. The ashes being analyzed, gave

Silica . . . . . . . 32

Phosphate of lime . . . . . 0.4

Carbonate of lime $\quad \cdot{ }^{\circ} \quad .15$

Phosphate of magnesia and soda $\quad$ - $\quad 29$

$8 \cdot 0$

It consists, then, of the following ingredients :-

Water . . 357.0

Vegetable fibre and animal matter $\quad 135.0$

Silica . • . $• . \quad 32$

Phosphate of lime . . . . 0.4

Carbonate of lime . . . . 1.5

Phosphate of magnesia and soda . $\quad 2.9$ 
principal constituents are the phosphates of lime and magnesia, carbonate of lime and silicate of potash; the first three of these preponderated in the corn, the latter in hay.

Thus in 1102 lbs. of horse-dung, we present to a field the inorganic substances contained in $6612 \mathrm{lbs}$. of hay, or $9146 \mathrm{lbs}$. of oats (oats containing 3.1 per cent. ashes according to De Saussure). This is sufficient to supply $1 \frac{1}{2}$ crop of wheat with potash and phosphates.

The excrements of cows, * black cattle, and sheep, contain phosphate of lime, common salt, and silicate of lime, the weight of which varies from 9 to 28 per cent., according to the fodder which the animal receives; the fresh excrements of the cow contain from 86 to 90 per cent. of water.

Human fæces have been subjected to an exact analysis by Berzelius. When fresh they contain, besides $\frac{3}{4}$ of their weight of water, nitrogen in very variable quantity, namely, in the minimum $1 \frac{1}{2}$, in the maximurn 5 per cent. In all cases, however, they were richer in this element than the excrements of other animals. Berzelius obtained by the incineration of 100 parts of dried excrements, 15 parts of ashes, which were principally composed of the phosphates of lime and magnesia.

The following quantitative organic analysis has recently been executed for the purpose of ascertain-

* It has been formerly stated (page 120), that all the potash contained in the food of a cow is again discharged in its excrements. The same also takes place with the other inorganic constituents of food, either when they are not adapted for assimilation, or when present in superabundant quantities. The value of manure may thus be artificially increased. We lately saw, for example, some cow-dung, sent by a farmer, who wished to ascertain the cause of its increased value. He had formerly employed this manure for his land, but with so little advantage that he found it more profitable to dry it, and use it as fuel. On inquiry, it was found, that his cows had been fed upon oil-cakes. This species of food is particularly rich in phosphates. More of these salts being present than were requisite for the purpose of assimilation, they were removed from the system in the form of excrementitious inatter, and in a condition adapted for the uses of plants. The fact that particular kinds of food enrich or impoverish the manure obtained from the cattle fed upon them, has repeatedly been observed. - ED. 
ing the proportion of carbon, nitrogen, and inorganic matter contained in fæces, in comparison with the food taken.* (Playfair.)

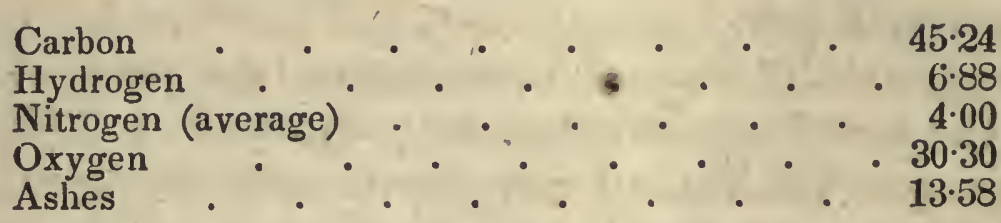

The inorganic matter contained in the excrements analyzed is nearly two per cent. less than that found by Berzelius; but the proportion always varies, according to the nature of the food.

It is quite certain, that the vegetable constituents of the excrements with which we manure our fields cannot be entirely without influence upon the growth of the crops on them, for they will decay, and thus furnish carbonic acid to the young plants. But it cannot be imagined that their influence is very great, when it is considered that a good soil is manured only once every six or seven years, or once every eleven or twelve years, ${ }^{,}$when sainfoin or lucern has been raised on it, that the quantity of carbon thus given to the land corresponds to only 5.8 per cent. of what is removed in the form of herbs, straw, and grain; and further that the rain-water received by a soil contains much more carbon in the form of carbonic acid than these vegetable constituents of the manure.

The peculiar action then, of the solid excrements is limited to their inorganic constituents, which thus restore to a soil that which is removed in the form of corn, roots, or grain. When we manure land with the dung of the cow or sheep, we supply it with silicate of potash and some salts of phosphoric acid. In human fæces we give it the phosphates of lime and magnesia; and in those of the horse, phosphate

* The details of the analysis are as follows : $-2 \cdot 356$ grammes left 0.320 gramme ashes after incineration; these consisted of the phosphate of lime and magnesia. 0.352 gramme yielded, on combustion with oxide of copper, 0.576 gram. carbonic acid, and 0.218 gram. water. (L. P.) 
of magnesia, and silicate of potash. In the straw which has served as litter, we add a further quantity of silicate of potash and phosphates; which, if the straw be putrefied, are in exactly the same condition in which they were before being assimilated.

It is evident, therefore, that the soil of a field will alter but little, if we collect and distribute the dung carefully; a certain portion of the phosphates, however, must be lost every year, being removed from the land with the corn and cattle, and this portion will accumulate in the neighborhood of large towns. The loss thus suffered must be compensated for in a wellmanaged farm, and this is partly done by allowing the fields to lie in grass. In Germany, it is considered that for every 100 acres of corn land, there must, in order to effect a profitable cultivation, be 20 acres of pasture-land, which produce annually, on an average, $551 \mathrm{lbs}$. of hay. Now assuming that the ashes of the excrements of the animals fed with this hay amount to 6.82 per cent., then $376 \mathrm{lbs}$. of the silicate of lime and phosphates of magnesia and lime must be yielded by these excrements, and will in a certain measure compensate for the loss which the corn-land had sustained.

The absolute loss in the salts of phosphoric acid, which are not again replaced, is spread over so great an extent of surface, that it scarcely deserves to be taken account of. But the loss of phosphates is. again replaced in the pastures by the ashes of the wood used in our houses for fuel.

We. could keep our fields in a constant state of fertility by replacing every year as much as we remove from them in the form of produce; but an increase of fertility, and consequent increase of crop. can only be obtained when we add more to them than we take away. It will be found, that of two: fields placed under conditions otherwise similar, the one will be most fruitful upon which the plants are enabled to appropriate more easily and in greater 16 
abundance those contents of the soil which are essential to their growth and development.

From the foregoing remarks it will readily be inferred, that for animal excrements, other subtances containing their essential constituents may be substituted. In Flanders, the yearly loss of the necessary matters in the soil is completely restored by covering the fields with ashes of wood or bones, which may or may not have been lixiviated ${ }^{*}$ and of which the greatest part consists of the phosphates of lime and magnesia. The great importance of manuring with ashes has been long recognised by agriculturists as the result of experience. So great a value, indeed, is attached to this material in the vicinity of Marburg and in the Wetterau, that it is transported as a manure from the distance of 18 or 24 miles. $t$ Its use will be at once perceived, when it is considered that the ashes, after having been washed with water, contain silicate of potash exactly in the same proportion as in straw (10 Si O $3+\mathrm{K} \mathrm{O}$.), and that their only other constituents are salts of phosphoric acid.

But ashes obtained from various kinds of trees are of very unequal value for this purpose; those from - oak-wood are the least, and those from beech the most serviceable. The ashes of oak-wood contain only traces of phosphates, those of beech the fifth part of their weight, and those of the pine and fir from 9 to 15 per cent. The ashes of pines from Norway contain an exceedingly small quantity of phosphates, namely, only 1.8 per cent. of phosphoric acid. (Berthier.) §

* Lixiviation signifies the removal by water of the soluble alkaline or saline matters in any earthy mixture; as from that of lime and potash, or from ashes to obtain a ley.

$\dagger$ Two well known agricultural districts; the first in Hesse-Cassel, the second in Hesse-Darmstadt. - Trans.

$\ddagger$ Ashes are used with great advantage on the light siliceous soil of Long Island, Connecticut, and various other places in the United States.

$\delta$ "The existence of phosphate of lime in the forest soils of the United States, is proved not only by its existence in the pollen of the pinus 
With every $110 \mathrm{lbs}$. of the lixiviated ashes of the beech which we spread over a soil, we furnish as much phosphates as 507 lbs. of fresh human excrements could yield. Again, according to the analysis of De Saussure, 100 parts of the ashes of the grain of wheat contain 32 parts of soluble, and 44.5 of insoluble phosphates, in all 76.5 parts. Now the ashes of wheat straw contain 11.5 per cent. of the same salts; hence with every $110 \mathrm{lbs}$. of the ashes of the beech, we supply a field with phosphoric acid sufficient for the production of $4210 \mathrm{lbs}$. of straw (its ashes being calculated at $4 \cdot 3$ per cent, De Saussure), or for 16-20000 lbs. of corn, the ashes of which amount, according to De Saussure, to 1.3 per cent.

Bone manure possesses a still greater importance in this respect. The primary sources from which the bones of animals are derived are, the hay, straw, or other substances which they take as food. Now if we admit that bones contain 55 per cent. of the phosphates of lime and magnesia (Berzelius), and that hay contains as much of them as wheat straw, it will follow that 8.8lbs. of bones contain as much phosphate of lime as 1102 lbs. of hay or wheatstraw, and $2.2 \mathrm{lbs}$. of it as much as $1102 \mathrm{lbs}$. of the grain of wheat or oats. These numbers express pretty nearly the quantity of phosphates which a soil yields annually on the growth of hay and corn. Now the manure of an acre of land with $44 \mathrm{lbs}$. of bone dust is sufficient to supply three crops of wheat,

abies (which is composed of 3 per cent. phosphate of lime and potash), but by its actual detection in the ashes of pines and other trees. - 10i parts of the ashes of wood of pinus abies give 3 per cent. phosphate of iron; 100 parts of the ashes of the coal of pinus sylvestris give 172 phosphate of lime, 0.25 phosphate of iron; 100 parts of ashes of oak coal give $7 \cdot 1$ phosphate of lime, $3 \cdot 7$ phosphate of iron; 100 parts of the ashes of bass wood give 54 phosphate of lime, $3 \cdot 2$ phosphate of iron; 100 parts of the ashes of birch wood give 7.3 phosphate of lime, 1.25 phosphate of iron; 100 parts of the ashes of oak wood give 1.8 phosphate of lime; 100 parts of the ashes of alder coal give 3.45 phosphate of lime, 9 phosphate of iron. These are the calculated results from Berthier's analyses." - Dr. S. L. Dana, in Report on a Reéxamination of the Economical Geology of Massuchusetts. 
clover, potatoes, turnips, \&c., with phosphates. But the form in which they are restored to a soil does not appear to be a matter of indifference. For the more finely the bones are reduced to powder, and the more intimately they are mixed with the soil, the more easily are they assimilated. The most easy and practical mode of effecting their division is to pour over the bones, in a state of fine powder, half of their weight of sulphuric acid diluted with three or four parts of water, and after they have been digested for some time, to add one hundred parts of water, and sprinkle this mixture over the field before the plough. In a few seconds, the free acids unite with the bases contained in the earth, and a neutral salt is formed in a very fine state of division. Experiments instituted on a soil formed from grauwacke, for the purpose of ascertaining the action of manure thus prepared, have distinctly shown that neither corn, nor kitchen-garden plants, suffer injurious effects in consequence, but that on the contrary they thrive with much more vigor.

It has also been found, that bones act more speedily and efficaciously after being boiled. This is probably owing to the removal of fatty matter, the presence of which impedes the putrefaction of the gelatin contained in them.

In the manufactories of glue, many hundred tons of a solution of phosphates in muriatic acid are yearly thrown away as being useless. It would be important to examine whether this solution might not be substituted for the bones. The free acid would combine with the alkalies in the soil, especially with the lime, and a soluble salt would thus be produced, which is known to possess a favorable action upon the growth of plants. This salt, muriate of lime (or chloride of calcium), is one of those compounds which attracts water from the atmosphere with great avidity, and in dry lands might advantageously supply the place of gypsum in decomposing carbonate of ammonia, with the formation of 
sal-ammoniac and carbonate of lime. A solution of bones in muriatic acid placed on land in autumn or in winter would, therefore, not only restore a necessary constituent of the soil, and attract moisture to it, but would also give it the power to retain all the ammonia which fell upon it dissolved in the rain during the period of six months.*

The ashes of brown coal $\dagger$ and peat often contain silicate of potash, $\ddagger$ so that it is evident, that these

* Immense quantities of bran are used in all print-works, for the purpose of clearing printed goods. After having served this purpose, it is thrown away. But the insoluble part of bran contains much phosphates of magnesia and soda; it would therefore be useful to preserve it as a manure. This has been done for some years in a farm with which I am connected, and its value as a manure has been found so great that it is much preferred to cow.dung. In some work's this waste bran is heaped up into little hillocks, which might be disposed of as a manure, instead of being an annoyance on account of the space which it occupies. - ED.

+ Brown coal. Braunkohle, Lignite has the structure and appearance of carbonized wood. It occurs abundantly in Germany ; in Hessia it forms beds 20 to 40 feet thick, and several square miles in extent. Fibrous and compact varieties occur near Bovey Tracey in England, where it is called Bovey coal. Small quantities are found at Gay Head, Massachusetts.

$\ddagger$ The following is the result of an analysis by Dr. C. T. Jackson, of peat from Lexington, Massachusetts. 100 grains, dried at $300^{\circ} \mathrm{F}$. weighed 74 grains, loss 26 grains, water. Burned in a platina crucible it left $5 \cdot 0$ ashes. The ashes yielded
Silex, . . . . . . . . 1.0
Alumina, iron, and manganese, . . . 0.6 .
Phosphate of lime, . . . . . . $\quad$. 3.0
Potash, traces.
$4 \cdot 6$

Peat from Watertown, Massachusetts, yielded 4.5 grains of ashes, which gave by analysis

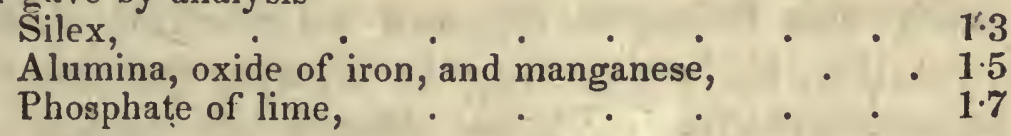

4.5

The vegetable matter amounted to 95.5 per cent., consisting of vegetable fibre, and apocrenic and crenic acids, in part combined with the bases obtained from its ashes. See Report on Rhode Island, p. 233.

Sroump muck contains the same ingredients as peat, but the vegetable matters are more finely divided, more soluble, and there is generally a larger proportion of earthy matters. It is formed of the fine particles of humus, washed out from the upland soils, and of the dead and decomposed leaves and roots of swamp plants.

'The pulpy matter of both peat and swamp muck consists chiefly of the apocrenic acid, in part combined with the earthy bases, and metallic oxides. The crenic acid is frequently united with lime and man- 
might completely replace one of the principal constituents of the dung of the cow and horse, and they contain also some phosphates. Indeed, they are much esteemed in the Wetterau as manure for meadows and moist land.

It is of much importance to the agriculturist, that he should not deceive himself respecting the causes which give the peculiar action to the substances just mentioned. It is known that they possess a very favorable influence on vegetation; and it is likewise certain that the cause of this is their containing a body, which, independently of the influence which it exerts by virtue of its form, porosity, and capability of attracting and retaining moisture, also assists in maintaining the vital processes in plants. If it be treated as an unfathomable mystery, the nature of this aid will never be known.

In medicine, for many centuries, the mode of action of all remedies was supposed to be concealed by the mystic veil of Isis, but now these secrets have been explained in a very simple manner. An unpoetical hand has pointed out the cause of the wonderful and apparently inexplicable healing virtues of the springs in Savoy; by which the inhabitants cured their goitre; it was shown that theycontain small quantities of iodine. In burnt sponges used for the same purpose; the same element was also detected. The extraordinary efficacy of Peruvian bark was found to depend on a small quantity of a crystalline body existing in it, viz. quinine; and the causes of the various effects of opium were detected in as many different ingredients of that drug.

Calico-printers used for a long time the solid excrements of the cow, in order to brighten and fasten colors on cotton goods; this material ap-

ganese; iron and magnesia occur in several of the peats analyzed. Phosphoric acid also exists in them, both in its free state, and in combination with lime and magnesia. In some peats Dr. J. found traces of oxalic acid and oxalates. Ibid., 210. See Appendx, for Peat 
peared quite indispensable, and its action was ascribed to a latent principle which it had obtained from the living organism. But since its action was known to depend on the phosphates contained in it, it has been completely replaced by a mixture of salts, in which the principal constituents are the phosphates of soda and lime.*

Now all such actions depend on a definite cause, by ascertaining which we place the actions themselves at our command.

It must be admitted as a principle of agriculture, that those substances which have been removed from a soil must be completely restored to it, and whether this restoration be effected by means of excrements, ashes, or bones, is in a great measure a matter of indifference. A time will come when fields will be manured with a solution of glass $\dagger$ (silicate of potash), with the ashes of burnt straw, and with salts of phosphoric acid, prepared in chemical manufactories, exactly as at present medicines are given for fever and goître.

There are some plants which require humus, and do not restore it to the soil by their excrements; whilst others can do without it altogether, and add humus to a soil which contains it in small quantity. Hence a rational system of agriculture would employ all the humus at command for the supply of the former, and not expend any of it for the latter ; and would in fact make use of them for supplying the others with humus.

We have now considered all that is requisite in a soil, in order to furnish its plants with the materials necessary for the formation of the woody fibre, the

* This mixture of salts is sold to calico-printers in large quantities under the naine of "dung substitute." It would be well worth experiment to try its effects as a manure upon land. Its cost is $3 \mathrm{~d}$. or $4 \mathrm{~d}$. per pound, and is not, therefore, dearer than nitrate of soda, which is now so extensively used. - ED.

t When glass contains a very large proportion of potash, it is soluble in boiling water; and by combination with other substances, silica becomes soluble in water. According to Dr. Jackson, crenic acid enables water to take it up. 
grain, the roots, and the stem, and now proceed to the consideration of the most important object of agriculture, viz. the production of nitrogen in a form capable of assimilation, - the production, therefore, of substances containing this element. The leaves, which nourish the woody matter, the roots, from which the leaves are formed, and which prepare the substances for entering into the composition of the fruit, and, in short, every part of the organism of a plant, contain azotized matter in very varying proportions, but the seeds and roots are always particularly rich in them.

Let us now examine in what manner the greatest possible production of substances containing nitrogen can be effected. Nature, by means of the atmosphere, furnishes nitrogen to a plant in quantity sufficient for its normal growth. Now its growth must be considered as normal, when it produces a single seed capable of reproducing the same plant in the following year. Such a normal condition would suffice for the existence of plants, and prevent their extinction, but they do not exist for themselves alone; the greater number of animals depend on the vegetable world for food, and by a wise adjustment of nature, plants have the remarkable power of converting, to a certain degree, all the nitrogen offered to them into nutriment for animals.

We may furnish a plant with carbonic, acid, and all the materials which it may require; we may supply it with humus in the most abundant quantity; but it will not attain complete development unless nitrogen is also afforded to it; a herb will be formed, but no grain; even sugar and starch may be produced but no gluten.

But when we give a plant nitrogen in considerable quantity, we enable it to attract with greater energy from the atmosphere the carbon which is necessary for its nutrition, when that in the soil is not sufficient; we afford to it a means of fixing the carbon of the atmosphere in its organism. 
We cannot ascribe much of the power of the excrements of black cattle, sheep, and horses, to the nitrogen which they contain, for its quantity is too minute. But that contained in the fæces of man is proportionably much greater, although by no means constant. In the fæces of the inhabitants of towns, for example, who feed on animal matter, there is much more of this constituent than in those of peasants, or of such people as reside in the country. The fæces of those who live principally on bread and potatoes are similar in composition and properties to those of animals.

All excrements have in this respect a very variable and relative value. Thus those of black cattle and horses are of great use on soils consisting of lime and sand, which contain no silicate of potash and phosphates; whilst their value is much less when applied to soils formed of argillaceous earth, basalt, granite, porphyry, clinkstone, and even mountainlimestone, because all these contain potash in considerable quantity. In such soils human excrements are extremely beneficial, and increase their fertility in a remarkable degree; they are, of course, as advantageous for other soils also; but for the manure of those first mentioned, the excrements of other animals are quite indispensable.

\section{OF URINE.}

We possess only one other natural source of manure which acts by its nitrogen, besides the fæces of animals, - namely, the urine of man and animals.

Urine is employed as a manure either in the liquid state, or with the fæces which are impregnated with it. It is the urine contained in them which gives to the solid fæces the property of emitting ammonia, a property which they themselves possess only in a very slight degree.

When we examine what substances we add to a 
soil by supplying it with urine, we find that this liquid contains in solution ammoniacal salts, uric acid (a substance containing a large quantity of nitrogen), and salts of phosphoric acid.

According to Berzelius 1000 parts of human urine contain :-

Urea

Free Lactic acid, ${ }^{*}$ Lactate of Ammonia, and animal matter not separable from them . . . 17.14

Uric acid . . . . . . . 1.00

Mucus of the bladder . . . . . . 032

Sulphate of Potash . . . . . . . . 377

Sulphate of Soda . . . . . . . $3 \cdot 16$

Phosphate of Soda . . . . . . 294

Phosphate of Ammonia . . . . . 1.65

Chloride of Sodium . . . . . . 4.45

Muriate of Ammonia . . . . . . 150

Phosphates of Magnesia and Lime . . . 1.00

Siliceous earth . . . . . . . 0.03

Water

$933 \cdot 00$

$1000 \cdot 00$

If we subtract from the above the urea, lactate of ammonia, free lactic acid, uric acid, the phosphate and muriate of ammonia; 1 per cent. of solid matter remains, consisting of inorganic salts, which must possess the same action when brought on a field, whether they are dissolved in water or in urine. Hence the powerful influence of urine must depend upon its other ingredients, namely, the urea and ammoniacal salts. The urea in human urine exists partly as lactate of urea, and partly in a free state. (Henry.) Now when urine is allowed to putrefy spontaneously, that is, to pass into that state in which it is used as manure, all the urea in combination with lactic acid is converted into lactate of ammonia, and that which was free, into volatile carbonate of ammonia.

In dung-reservoirs well constructed and protected from evaporation, this carbonate of ammonia is retained in the state of solution, and when the putre-

* Lactic acid has been found in most animal fluids and in several plants. It was first obtained from sour milk, heñce its name from the Latin lac, milk. 
fied urine is spread over the land, a part of the ammonia will escape with the water which evaporates, but another portion will be absorbed by the soil, if it contains either alumina or iron; but in general only the muriate, phosphate, and lactate of ammonia remain in the ground. It is these alone, therefore, which enable the soil to exercise a direct influence on plants during the progress of their growth, and not a particle of them escapes being absorbed by the roots.

On account of the formation of this carbonate of ammonia the urine becomes alkaline, although it is acid in its natural state. When it is lost by being volatilized in the air, which happens in most cases, the loss suffered is nearly equal to one half of the weight of the urine employed, so that if we fix it, that is, if we deprive it of its volatility, we increase its action twofold. The existence of carbonate of ammonia in putrefied urine long since suggested the manufacture of sal-ammoniac from this material. When the latter salt possessed a high price, this manufacture was even carried on by the farmer. For this purpose the liquid obtained from dunghills was placed in vessels of iron, and subjected to distillation; the product of this distillation was converted into muriate of ammonia by the common method. (Demachy.) But it is evident that such a thoughtless proceeding must be wholly relinquished, since the nitrogen of $100 \mathrm{lbs}$. of sal-ammoniac (which contains 26 parts of nitrogen) is equal to the quantity of nitrogen contained in 1200 lbs. of the grain of wheat, $1480 \mathrm{lbs}$. of that of barley, or $2755 \mathrm{lbs}$. of hay. (Boussingault.)

The carbonate of ammonia formed by the putrefaction of urine, can be fixed or deprived of its volatility in many ways.

If a field be strewed with gypsum, and then with putrefied urine or the drainings of dunghills, all the carbonate of ammonia will be converted into the sulphate which will remain in the soil. 
But there are still simpler means of effecting this purpose;-gypsum, chloride of calcium (bleaching salts), sulphuric or muriatic acid, and super-phosphate of lime, are all substances of a very low price, and completely neutralize the urine, converting its ammonia into salts which possess no volatility.

If a basin, filled with concentrated muriatic acid, is placed in a common necessary, so that its surface is in free communication with the vapors which rise from below, it becomes filled after a few days with crystals of muriate of ammonia. The ammonia, the presence of which the organs of smell amply testify, combines with the muriatic acid and loses entirely its volatility, and thick clouds or fumes of the salt newly formed hang over the basin. In stables the same may be seen. The ammonia-that escapes in this manner is not only entirely lost, as far as our vegetation is concerned, but it works also a slow, though not. less certain destruction of the walls of the building. For when in contact with the lime of the mortar, it is converted into nitric acid, which gradually dissolves the lime. The injury thus done to a building by the formation of the soluble nitrates, has received (in Germany) a special name, - salpeterfrass.

The ammonia emitted from stables and necessaries is always in combination with carbonic acid. Carbonate of ammonia and sulphate of lime (gypsum) cannot be brought together at common temperatures, without mutual decomposition. The ammonia enters into combination with the sulphuric acid, and the carbonic acid with the lime, forming compounds which are not volatile, and consequently destitute of all smell. Now, if we strew the floors of our stables, from time to time, with common gypsum, they will lose all their offensive smell, and none of the ammonia which forms can be lost, but will be retained in a condition serviceable as manure.

With the exception of urea, uric acid contains more nitrogen than any other substance generated 
by the living organism; it is soluble in water, and can be thus absorbed by the roots of plants, and its nitrogen assimilated in the form of ammonia, and of the oxalate, hydrocyanate, or carbonate of ammonia.

It would be extremely interesting to study the transformations which uric acid suffers in a living plant. For the purpose of experiment, the plant should be made to grow in charcoal powder previously heated to redness, and then mixed with pure uric acid. The examination of the juice of the plant, or of the component parts of the seed or fruit, would be a means of easily detecting the differences.

\section{NIGHT-SOIL.}

In respect to the quantity of nitrogen contained in excrements, 100 parts of the urine of a healthy man are equal to 1300 parts of the fresh dung of a horse, according to the analyses of Macaire and Marcet, and to 600 parts of those of a cow. Hence it is evident that it would be of much importance to agriculture if none of the human urine were lost. The powerful effects of urine as a manure are well known in Flanders, ${ }^{*}$ but they are considered invaluable by the Chinese, who are the oldest agricultural people we know. Indeed, so much value is attached to the influence of human excrements by these people, that laws of the state forbid that any of them should be thrown away, and reservoirs are placed in every house, in which they are collected with the greatest care. No other kind of manure. is used for their corn-fields. $\dagger$

* See the article "On the Agriculture of the Netherlands," Journ. Royal Agri. Soc., Vol. II. part 1, page 43, for much interesting informa-. tion on this subject.

+ Davis, in his History of China, states that every substance convertible into manure is diligently husbanded. "The cakes that remain: after the expression of their vegetable oils, horns and hoofs reduced to powder, together with soot and ashes, and the contents of common 


\section{China is the birthplace of the experimental art; the incessant striving after experiments has con-}

sewers, are much used. The plaster of old kitchens, which in China have no chimneys but an opening at the top, is much valued; so that they will sometimes put a new plaster on a kitchen for the sake of the old." The ammonia contained in the fuel forms nitrate of lime with the lime in the mortar. "All sorts of hair are used as a manure, and barbers' shavings are carefully appropriated to that purpose. The annual produce must be considerable in a country where some hundred millions of heads are kept constantly shaved. Dung of all animals, but more especially night-soil, is esteemed above all others. Being sometimes formed into cakes, it is dried in the sun, and in this state becomes an object of sale to farmers, who dilute it previous to use: They construct large cisterns or pits, lined with lime plaster, as well as earthen tubs, sunk into the ground, with straw over them to prevent evaporation, in which all kinds of veretables and animal refuse are collected. These being diluted with a sufficient quantity of liquid, are left to undergo the putrefactive fermentation, and then applied to the land. In the case of every thing except rice, the Chinese seem to manure the plant itself rather than the soil, supplying it copiously with their liquid preparation."

"The Chinese husbandman," observes Sir G. Staunton, (Embassy, Vol. II.,) "always steeps the seeds he intends to sow in liquid manure, until they swell, and germination begins to appear, which experience has taught him will have the effect of hastening the growth of plants, as well as of defending them against the insects hidden in the ground in which the seeds are sown. To the roots of plants and fruit-trees, the Chinese farmer applies liquid manure likewise." *

Lastly, we extract the following from a communication to Professor Webster, of Harvard College, United States. - "Human urine, is, if possible, more husbanded by the Chinese than niglit-soil for manure; every farm, or patch of land for cultivation, has a tank, where all substances convertible into manure are carefully deposited, the whole made liquid by adding urine in the proportion required, and invariably applied in that state." This is exactly the process followed in the Netherlands: see Outlines of Flemish Husbandry, page 22.

"The business of collecting urine and night-soil employs an immense number of persons, who deposit tubs in every house in the cities for the reception of the urine of the inmates, which vessels are removed daily, with as much care as our farmers remove their honey from the hives."

When we consider the immense value of night-soil as a manure, it is quite astounding that so little attention is paid to preserve it. The quantity is immense which is carried down by the drains in London to the River Thames, serving no other purpose than to pollute its waters. It has been shown, by a very simple calculation, that the value of the manure thus lost amounts annually to several millions of pounds sterling. A substance, which by its putrefaction generates miasmata, may, by artificial means, be rendered totally inoffensive, inodorous, and transportable, and yet prejudice prevents these means being resorted to. - ED.

* These statements are confirmed by others, which have been kindly communicated to me by a gentleman whose opportunities for observation during a residence in China of several years, were ample, and whose liberality and devotion to agriculture and horticulture have already conferred upon the community results of great interest and value. - See APPENDix. 
ducted the Chinese a thousand years since to discoveries, which have been the envy and admiration of Europeans for centuries, especially in regard to dyeing and painting, and to the manufactures of porcelain, silk, and colors for painters. These we were long unable to imitate, and yet they were discovered by them without the assistance of scientific principles; for in the books of the Chinese we find recipes and directions for use, but never explanations of processes.

Half a century sufficed to Europeans not only to equal but to surpass the Chinese in the arts and manufactures, and this was owing merely to the application of correct principles deduced from the study of chemistry. But how infinitely inferior is the agriculture of Europe to that of China! The Chinese are the most admirable gardeners and trainers of plants, for each of which they understand how to prepare and apply the best-adapted manure. The agriculture of their country is the most perfect in the world; and there, where the climate in the most fertile districts differs little from the European, very little value is attached to the excrements of animals. With us, thick books are written, but no experiments instituted; the quantity of manure consumed by this and that plant is expressed in hundredth parts, and yet we know not what manure is!

If we admit that the liquid and solid excrements of man amount on an average to $1 \frac{1}{2} \mathrm{lb}$. daily $\left(\frac{5}{4} \mathrm{lb}\right.$. of urine and $\frac{1}{4} \mathrm{lb}$. fæces), and that both taken together contain 3 per cent. of nitrogen, then in one year they will amount to $547 \mathrm{lbs}$., which contain $16.41 \mathrm{lbs}$. of nitrogen, a quantity sufficient to yield the nitrogen of $800 \mathrm{lbs}$. of wheat, rye, oats, or of 900 lbs. of barley.

(Boussingault.)

This is much more than it is necessary to add to an acre of land in order to obtain, with the assistance of the nitrogen absorbed from the atmosphere, the richest possible crop every year. Every town and farm might thus supply itself with the manure, which, 
besides containing the most nitrogen, contains also the most phosphates; and if rotation of the crops were adopted, they would be most abundant. By using, at the same time, bones and the lixiviated ashes of wood, the excrements of animals might be completely dispensed with.

When human excrements are treated in a proper manner, so as to remove the moisture which they contain without permitting the escape of ammonia; they may be put into such a form as will allow them to be transported even to great distances.

This is already attempted in many towns, and the preparation of night-soil for transportation constitutes not an unimportant branch of industry. But the manner in which this is done is the most injudicious which could be conceived. In Paris, for example, the excrements are preserved in the houses in open casks, from which they are collected and placed in deep pits at Montfaucon, but are not sold until they have attained a certain degree of dryness by evaporation in the air. But whilst lying in the receptacles appropriated for them in the houses, the greatest part of their urea is converted into carbonate of ammonia; lactate and phosphate of ammonia are also formed, and the vegetable matters contained in them putrefy; all their sulphates are decomposed, whilst their sulphur forms sulphuretted hydrogen and hydro-sulphate of ammonia. The mass, when dried by exposure to the air, has lost more than half of the nitrogen which the excrements originally contained; for the ammonia escapes into the atmosphere along with the water which evaporates; and the residue now consists principally of phosphate of lime, with phosphate and lactate of ammonia, and small quantities of urate of magnesia and fatty matter. Nevertheless, it is still a very powerful manure, but its value as such would be twice or four times as great, if the excrements before being dried.were neutralized with a cheap mineral acid. 
In other manufactories of manure the night-soil, whilst still soft, is mixed with the ashes of wood, or with earth, ${ }^{*}$ both of which substances contain a large quantity of caustic lime, by means of which a complete expulsion of all its ammonia is effected, and it is completely deprived of smell. But such a residue applied as manure can act only by the phosphates which it still contains, for all the ammoniacal salts have been decomposed and their ammonia expelled.

The preparation of night-soil is now carried on in London to a considerable extent. Owing to the variable nature of the climate, artificial means are employed in its desiccation. The night-soil, after being subjected to one or other of the modes of treatment described below, is placed upon iron plates heated by means of furnaces.

As soon as the night-soil is collected, it is placed in large broad trenches, until a sufficient quantity is accumulated for the purposes of the manufacturer. But here it undergoes the same process of putrefaction to which allusion has been made, and acquires a peculiarly offensive smell from the evolution of sulphuretted hydrogen and other gases, which are observed to escape. Unless some means be employed, at this stage of the process, to retain the ammonia, it escapes into the atmosphere in the form of a carbonate. Various methods have been proposed to effect this purpose. Some manufacturers mix the night-soil with chloride of lime, and evaporate off the water by the aid of heat. This possesses the advantage of depriving the excrements of smell, and at the same time partially fixes the ammonia which would otherwise escape. Chloride of lime always contains a considerable excess of lime; hence part of the ammonia contained in the night-soil is expelled by means of it.

More simple and economical methods might be employed. A patent, which has been taken out for States.

* This is practised in the vicinity of large cities in the United 
the preparation of this useful manure states in its specification, that the night-soil is to be mixed with calcined mud and finely-divided charcoal. By this means, the smell is completely and instantaneously removed, and the ammonia retained by virtue of the affinity, which alumina and charcoal exert for that compound. This plan is both simple and efficacious, but the ammonia is apt to be expelled by the application of the heat employed in drying the manure. The addition of a cheap mineral acid to the nightsoil, before admixture with these ingredients, would materially improve both of the above processes.

It would no doubt be highly advantageous in the preparation of manures, to prepare them so that they contained all the ingredients necessary for the supply of the plants to which they are applied. But these will of course vary according to the nature of the soils and plants for which they are intended. Thus bones, soap-boilers' waste, nitrate of soda, and ashes of wood, will often be found to form advantageous additions. Sulphate of magnesia (Epsom salts) would, in most cases, form an invaluable ingredient in prepared night-soil. (See Supplementary Chapter on Soils.) 'The products of the decomposition proceeding from the action of this salt upon night-soil are, sulphate of ammonia, phosphate of magnesia, and the double phosphate of magnesia and ammonia. Now all these salts exert a very favorable influence upon vegetation, and the phosphate of magnesia is, in many cases, perfectly indispensable to the growth and development of certain plants. This suggestion is well worthy of the attention of the farmer.

Perhaps the best and most practical method of fixing the ammoniacal salts of urine and night-soil, is to mix them with the ashes of peat or coal. When the latter are employed, care must be taken to select such as are of a porous, earthy consistence. The ashes both of peat and coal contain in general magnesia; hence their value as an ingredient of prepared 
night-soil. When magnesia is not present, it will be necessary to add some magnesian limestone or Epsom salts. The night-soil should be mixed thoroughly with the ashes, and exposed to the air to dry. The disagreeable smell is thus quickly removed, and a pulverulent manure obtained, which can be applied to the fields with facility.*

Animal charcoal, which has served for the discoloration of sugar, possesses the property of removing the offensive smell of night-soil, and is of itself an admirable manure. In cases where it can be procured with facility, it will be found to add to the efficacy of the latter. $f$

\section{GUANO.}

The sterile soils of the South American coast are manured with a substance called guano, consisting of urate of ammonia and other ammoniacal salts, by the use of which a luxuriant vegetation and the richest crops are obtained. Guano has lately been imported in considerable quantity into Liverpool and several other English ports, and is now experimentally employed as a manure by English agriculturists. A consideration of its composition and mode of action cannot, therefore, fail to be acceptable.

Much speculation has arisen as to the true origin of Guano, $\ddagger$ but the most certain proof is now afforded, that it has been produced by, the accumula-

* Night soil deprived of its odor and rendered portable is termed poudrette. One mode of preparing it, practised in France, is by boiling the refuse matter of slaughter-houses, by steam, into a thick soup and then mixing the whole into a stiff paste with sifted coal ashes, and drying. It is almost one half animal matter. If putrefaction should have begun, the addition of ashes, sweetens the whole, and the prepared "animalized coal," as it is termed, is as sweet to the nose, as garden mould. - Dana.

+ For an account of Mr. Daniell's artificial manure, see Appendix.

$\$$ Much of the information regarding Guano here given is extracted from a paper in Liebig's Annalen, xxxvii. 3, 291. 
tion of the excrements of innumerable sea-fowl, which inhabit the islands upon which it is found. Meyen, the latest writer upon this subject, completely coincides with this opinion; for he says*_" Their number is Legion; they completely cloud the sun, when they rise from their resting-place in the morning in flocks of miles in length." Yet, notwithstanding their' great number, thousands of years must have elapsed, before the excrements could have accumulated to such a thickness as they possess at present. Guano has been used by the Peruvians as a manure since the twelfth century; and its value was considered so inestimable, that the government of the Incas issued a decree, by which capital punishment was inflicted upon any person found destroying the fowl on the Guano islands. Overseers were also appointed over each province, for the purpose of insuring them further protection. Under this state of things, the accumulation of the excrements may have well taken place. All these regulations are, however, now abandoned.t Rivero states, that the annual consumption of guano for the purposes of agriculture amounts to 40,000 fanegas. The increase of crops obtained by the use of guano is very remarkable. According to the same authority, the crop of potatoes is increased 45 times by means of it, and that of maize 35 times. The manner of applying the manure is singular. Thus in Arica, where so much pepper (Capsicum baccatum) is cultivated, each plant is manured three times: first upon the appearance of the roots, second upon that of the leaves, and lastly upon the formation of the fruit. (Humboldt.) From this it will be observed, that the Peruvians follow the plan of the Chinese, in manuring the plant rather than the soil. 'The composition of guano points out how admirably it is fitted for a manure; for not only does it contain

* Reise um die Erde, B. i. S. 434.

† Garcilaso, Historie de los Yncas, Vol. I. p. 134. 
ammoniacal salts in abundance, but also those inorganic constituents which are indispensable for the development of plants.

The most recent analysis is that of Völckel, who found it to consist of

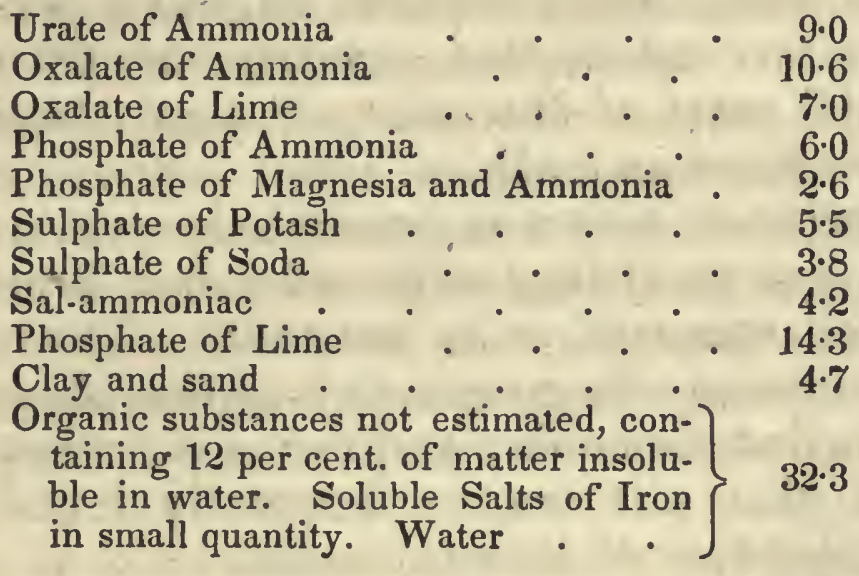

100.0

It will be observed from the above analysis, that urea does not enter into the composition of guano. The uric acid of the excrements must have been decomposed into oxalic acid and ammonia. The soluble substances contained in guano amount to half its weight. It is singular that we do not find nitrates amongst the ingredients which compose it. Guano possesses a urinous smell, precisely similar to that perceived on the evaporation of urine. The - experiments upon the efficacy of this manure in England have not yet been sufficiently multiplied to enable us to judge whether or not its virtues have been overrated.

The corn-fields in China receive no other manure than human excrements. But we cover our fields every year with the seeds of weeds, which from their nature and form pass undigested along with the excrements through animals, without being deprived of their power of germination, and yet it is considered surprising that where they have once flourished, they cannot again be expelled by all our endeavors: we think it very astonishing, while we really sow them ourselves every year. A famous 
botanist, attached to the Dutch embassy to China, could scarcely find a single plant on the corn-fields of the Chinese, except the corn itself.*

The urine of horses contains less nitrogen and phosphates than that of man. According to Fourcroy and Vauquelin it contains only five per cent. of solid matter, and in that quantity only 0.7 of urea; whilst 100 parts of the urine of man contain more than four times as much.

The' urine of a cow is particularly rich in salts of potash; but according to Rouelle and Brande, it is almost destitute of salts of soda. The urine of swine contains a large quantity of the phosphate of magnesia and ammonia; and hence it is that concretions of this salt are so frequently found in the urinary bladders of these animals.

It is evident, that if we place the solid or liquid excrements of man or the liquid excrements of animals on our land, in equal proportion to the quantity of nitrogen removed from it in the form of plants, the sum of this element in the soil must increase every year; for to the quantity which we thus supply, another portion is added from the atmosphere. The nitrogen which we export as corn and cattle, and which is thus absorbed by large towns, serves only to benefit other farms, if we do not replace it. A farm which possesses no pastures, and not fields sufficient for the cultivation of fodder, requires manure containing nitrogen to be imported from elsewhere, if it is desired to produce a full crop. In large farms, the annual expenditure of nitrogen is completely replaced by means of the pastures.

The only absolute loss of nitrogen, therefore, is limited to the quantity which man carries with him to his grave; but this at the utmost cannot amount to more than 3 lbs. for every individual, and is being collected during his whole life. Nor is this quantity

* Ingenhouss on the Nutrition of Plants, page 129 (German edition). 
lost to plants, for it escapes into the atmosphere as ammonia during the putrefaction and decay of the body.

A high degree of culture requires an increased supply of manure. With the abundance of the manure, the produce in corn and cattle will augment, but must diminish with its deficiency.

From the preceding remarks it must be evident, that the greatest value should be attached to the liquid excrements of man and animals, when a manure is desired which shall supply nitrogen to the soil. The greatest part of a superabundant crop, or, in other words, the increase of growth which is in our power, can be obtained exclusively by their means.

When it is considered that with every pound of ammonia which evaporates a loss of 60 lbs. of corn is sustained, and that with every pound of urine a pound of wheat might be produced, the indifference with which these liquid excrements are regarded is quite incomprehensible. In most places only the solid excrements impregnated with the liquid are used, and the dunghills containing them are protected neither from evaporation nor from rain. The solid excrements contain the insoluble, the liquid all the soluble phosphates, and the latter contain likewise all the potash which existed as organic salts in the plants consumed by the animals.

Fresh bones, wool, hair, hoofs, and horn, are manures containing nitrogen as well as phosphates, and are consequently fit to aid the process of vegetable life. All animal matter is fitted for the same purpose. Butchers' offal, such as the blood and intestines of animals, form a most powerful manure. It is in general necessary to dilute such manure by admixture with other kinds less powerful in their action.

One hundred parts of dry bones contain from 32 to 33 per cent. of dry gelatine; now supposing this to contain the same quantity of nitrogen as animal 
glue, viz., 5.28 per cent., then 100 parts of bones must be considered as equivalent to 250 parts of human urine.

Bones may be preserved unchanged for thousands of years, in dry or even in moist soils, provided the access of rain is prevented; as is exemplified by the bones of antediluvian animals found in loam or gypsum, the interior parts. being protected by the exterior from the action of water. But they become warm when reduced to a fine powder, and moistened bones generate heat and enter into putrefaction; the gelatine which they contain is decomposed, and its nitrogen converted into carbonate of ammonia and other ammoniacal salts, which are retained in a great measure by the powder itself. (Bones burnt till quite white, and recently heated to redness, absorb 7.5 times their volume of pure ammoniacal gas.)

\section{ARTIFICIAL MANURES.}

WE have now examined the action of the animal or natural manures upon plants; but it is evident, that if artificial manures contain the same constituents, they will exercise a similar action upon the plants to which they are applied. We shall only notice here one or two of those principally employed.

Since it has been ascertained that animal manures act (as far as the formation of organic matter is concerned) only by the ammonia which they contain, attention has been devoted by chemists to discover a more economical means of presenting this ammonia to plants. The water which distils from the retorts in the preparation of coal gas is strongly charged with this alkali, but is at the same time mixed with tar and other empyreumatic impurities. It has been customary to allow the tarry matter to subside, and decant off the clear, supernatant liquor. This liquor, 
being diluted to such a degree as to be tasteless, is applied as a manure to the field.*

Now, the ammoniacal liquor of the gas-works contains the ammonia in the form of carbonate and hydro-sulphate of ammonia (sulphuret of ammonium). The latter compound is a deadly poison to vegetables, nor can we conceive that by dilution its properties can be changed. The carbonate of ammonia is volatile, and escapes into the atmosphere. To obviate this latter inconvenience and render it more transportable, it has been proposed to convert the carbonate into the sulphate, by means of gypsum. + But this does not remove the hydro-sulphate. A more simple and efficacious method is to add a solution of sulphate of iron (the green vitriol of the shops) to the liquor, until no further precipitation ensues. Sulphuret and carbonate of iron are thus formed, and the whole of the ammonia enters into combination with the sulphuric acid, and forms sulphate of ammonia. Care must be taken to avoid too great an excess of sulphate of iron; and the liquor thus prepared should be freely exposed to the air to promote the oxidation.

'The liquor still, however, contains empyreumatic matters, which are injurious to plants. These may be removed by evaporating the liquor to dryness, and heating the residue to incipient redness. By this means they are rendered insoluble, and the sulphate of ammonia is not affected, unless the heat has been carried too far. The liquor properly diluted has been found very advantageous, even without the removal of the empyreumatic matter.

* Mr. Blake, who has charge of the gas-work in Boston, informs me, that one chaldron ( $2700 \mathrm{lbs}$. of Pictou coal, yields, on the average, 33 gallons of ammoniacal liquor containing about 5 per cent. of dry ammonia; and by passing the gases generated from this quantity of coal through a solution of proto-sulphate of iron, he has obtained in addition 24 gallons of a solution containing about 4 per cent. of dry ammonia. About 4 chaldrons of coal are used per diem, at the gas-works in Boston, and 200 gallons of liquor, containing from 4 to 5 per cent. of am.. monia, could be furnished daily at sinall cost. - $W$.

$\uparrow$ Three Lectures on Agriculture, by Dr. Daubeny, page 87. 
Nitrate of soda has lately engaged much attention, and is supposed to exert its favorable action upon vegetation by yielding nitrogen to those constituents of plants which contain it. The experiments which have hitherto been instituted with this manure do not warrant us in concluding with positive certainty that it is the nitrogen alone to which it owes its efficacy, but they certainly render this a plausible explanation of its virtues. Thus Mr. Pusey, the late able president of the Royal Agricultural Society, has shown, that the same effects are produced by putrefied urine, soot, gas-liquor, and nitrate of soda.* Now the three former act by virtue of the ammonia which enters into their composition. The usual effects produced by these and nitrate of soda are to increase the intensity of the green coloring matter, to augment the quantity of straw, but to produce a light grain. Mr. Hyett $\dagger$ has communicated the results of an analysis of two samples of wheat grown under similar circumstances, one of which had been treated with nitre, the other not. The former contained 23.25 per cent. of gluten, and 1.375 of albumen; the latter only 19 per cent. of gluten, and 0.62 of albumen. Here the azotized matters appear to have considerably increased in quantity. There is nothing opposed to the supposition that nitric acid may be decomposed by plants, and its nitrogen assimilated. We find that vegetables possess the power of decomposing carbonic acid, and of appropriating its carbon for their own use. Now this acid is infinitely more difficult to decompose than nitric acid. But there are other circumstances which oppose the adoption of the view that nitrate of soda acts by virtue of the nitrogen which enters into its composition. Were this the case, the action should be more uniform than it has hitherto been found to be. On some soils the salt does not possess the smallest influence; whilst on

* Journal of the Royal Agricultural Society, Vol. II. p. 123.

$\dagger$ Ibid., Vol. II. p. 143. 
others it affords great benefit. We can only furnish an explanation of this seeming caprice by a reference to the chemical composition of the soil upon which it is applied. If the advantages attending the application of nitrate of soda are due to the alkaline base which it contains, then it is evident that this manure can be of small value on soils containing a quantity of alkalies sufficient for the purposes of the plants grown upon them; whilst, on the other hand, such as are deficient in these must experience benefit through its means.* In certain cases in which nitrate of soda has failed, nitrate of potash (common saltpetre) has been very successful. Analyses of wheat grown with nitrate of soda and nitrate of potash would be of interest, in order to determine whether a mutual substitution of their respective bases is effected. It is to be hoped that future experiments will throw more light upon the action of this interesting manure, for theory cannot be satisfied with those already existing. It has been usual to employ a less quantity by weight of nitrate of potash than of nitrate of soda. This procedure seems rather empirical, for unless sanctioned by experience, it would $\dot{a}$ priori appear to be better to add the greatest quantity of that salt which possesses the highest equivalent. Now the equivalent of nitrate of potash is considerably higher than that of nitrate of soda.

Charcoal in a state of powder must be considered as a very powerful means of promoting the growth of plants on heavy soils, and particularly on such as consist of argillaceous earth. $\nmid$

* General Sir Howard Elphinstone informs me, that he found carbonate of soda (soda ash) an excellent manure for his land. The crops obtained by means of it presented the same general characters as those manured with nitrate of potash, and exhibited a greater intensity of color. If this is found uniformly to be the case, it will very much strengthen the supposition that the action of nitrate of soda is due to its alkaline constituent. - ED.

$\dagger$ For much valuable information on the subject of manures, see "Agricultural Chemistry," Vol. VIII. of Sir H. Davy's collected Works. 
Ingenhouss proposed dilute sulphuric acid as a means of increasing the fertility of a soil. Now, when this acid is sprinkled on calcareous soils, gypsum (sulphate of lime) is immediately formed, which of course prevents the necessity of manuring the soils with this material. 100 parts of concentrated sulphuric acid diluted with from 800 to 1000 parts of water, are equivalent to 176 parts of gypsum.

\section{SUPPLEMENTARY CHAPTER.}

ON THE CHEMICAL CONSTITUENTS OF SOILS.

The fertility of a soil is much influenced by its physical properties, such as its porosity, color, attraction for moisture, or state of disintegration. But independently of these conditions, the fertility depends upon the chemical constituents of which the soil is composed.

We have already shown, at considerable length, that those alkalies, earths, and phosphates, which constitute the ashes of plants, are perfectly indispensable for their development; and that plants cannot flourish upon soils from which these compounds are absent. The necessity of alkalies for the vital processes of plants will be obvious, when we consider that almost all the different families of plants are distinguished by containing certain acids, differing very much in composition; and further, that these acids do not exist in the juice in an isolated state, but generally in combination with certain alkaline or earthy bases. The juice of the vine contains tartaric acid, that of the sorrel oxalic acid. It is quite obvious, that a peculiar action must be in operation in the organism of the vine and sorrel, by means of which the generation of tartaric and oxalic acid is effected; and also that the same action must exist in all plants of the same genus. 
A similar cause forces corn-plants to extract silicic acid from the soil. The number of acids found in different plants is very numerous, but the most common are those which we have already mentioned; to which may be added acetic, malic, citric, aconitic, maleic, kinovic acids, \&c.

When we observe that the proper acids of each family of plants are never absent from it, we must admit that the plants belonging to that family could not attain perfection, if the generation of their peculiar acids were prevented. Hence, if the production of tartaric acid in the vine were rendered impossible, it could not produce grapes, or in other words, would not fructify. Now the generation of organic acids is prevented in the vine, and, indeed, in all plants which yield nourishment to men and animals, when alkalies are absent from the soil in which they grow. The organic acids in plants are very rarely found in a free state; in general, they are in combination with potash, soda, lime, or magnesia. Thus, silicic acid is found as silicate of potash, acetic acid as acetate of potash or soda, oxalic acid as oxalate of potash, soda, or lime, tartaric acid as bitartrate of potash, \&c. The potash, soda, lime, and magnesia in these plants are, therefore, as indispensable for their existence as the carbon from which their organic acids are produced.

In order not to form an erroneous conclusion regarding the processes of vegetable nutrition, it must be admitted that plants require certain salts for the sustenance of their vital functions, the acids of which salts exist either in the soil (such as silicic or phosphoric acids) or are generated from nutriment derived from the atmosphere. Hence, if these salts are not contained in the soil, or if the bases necessary for their production be absent, they cannot be formed; or in other words, plants cannot grow in such a soil. The juice, fruit, and leaves of a plant cannot attain maturity, if the constituents necessary for their formation are wanting, and salts must be 
viewed as such. These salts do not, however, occur simultaneously in all plants. Thus, in saline plants, soda is the only alkali found; in corn plants, lime and potash form constituents. Several contain both soda and potash, some both potash and lime; whilst others contain potash and magnesia. The acids vary in a similar manner. Thus one plant may contain phosphate of lime, a second, phosphate of magnesia, a third, an alkali combined with silicic acid, and a fourth, an alkali in combination with a vegetable acid. The respective quantities of the salts required by plants are very unequal. The aptitude of a soil to produce one, but not another kind of plant, is due to the presence of a base which the former requires, and the absence of that, indispensable for the development of the latter. Upon the correct knowledge of the bases and salts requisite for the sustenance of each plant, and of the composition of the soil upon which it grows, depends the whole system of a rational theory of agriculture; and that knowledge alone can explain the process of fallow, or furnish us with the most advantageous methods of affording plants their proper nourishment.

Give, - so says the rational theory, - to one plant such substances as are necessary for its development, but spare those, which are not requisite, for the production of other plants that require them.

It is the same with regard to these bases as it is with the water which is necessary for the roots of various plants. Thus, whilst one plant flourishes luxuriantly in an arid soil, a second requires much moisture, a third finds necessary this moisture at the commencement of its development, and a fourth (such as potatoes) after the appearance of the blossom. It would be very erroneous to present the same quantity of water to all plants indiscriminately. Yet this obvious principle is lost sight of in the manuring of plants. An empirical system of agriculture has administered the same kind of manures 
to all plants; or when a selection has been made, it was not based upon a knowledge of their peculiar characters or composition.

The cost of labor in England has given rise to the production of much ingenuity in the invention of machines, which have produced improvements in the mode of application of manures. In order to use these with advantage, pulverulent manures are employed, instead of the common stable manure, which is generally mixed with much straw.

The necessity for such forms of manure naturally suggested the employment of bone dust, dried dung, lime, ashes, \&c. Now, although by these means the necessary phosphates are furnished to a soil, and solid animal excrements rendered unnecessary, they have led to the neglect of the liquid excrements, that is, of the urine of men and animals, which is thus completely lost to agriculture. For although the meadows receive, during autumn and winter, when cattle are fed upon them, the solid and liquid excrements of these animals, yet the urine of man, into which all the nitrogenous constituents of animals are finally deposited, is completely lost to the fields. This most important of all manures, so properly estimated in Flanders, Germany, and China, is altogether lost to the English agriculturist. In large towns'it is either allowed to run into the rivers, or sink into the ground in such a manner as to be of no benefit to the regetable kingdom.

The most important growth in England is that of wheat; then of barley, oats, beans, and turnips. Potatoes are only cultivated to a great extent in certain localities ; rye, beet-root, and rape-seed, not very generally. Lucern is only known in a few districts, whilst red clover is found universally. Now, the selection of inorganic manures for these plants may be fixed upon by an examination of the composition of their ashes. Thus wheat must be cultivated in a soil rich in silicate of potash. If this soil is formed from feldspar, mica, basalt, clinkstone, or indeed of any. 
minerals which disintegrate with facility, crops of wheat and barley may be grown upon it for many centuries in succession. But, in order to support an uninterrupted succession, the annual disintegration must be sufficiently great to render soluble a quantity of silicate of potash sufficient for the supply of a full crop of wheat or barley. If this is not the case, the soil must either be allowed to lie fallow from time to time, or plants may be cultivated upon it which contain little silicate of potash, or the roots of which are enabled to penetrate deeper into the soil than corn plants in search of this salt. During this interval of repose, the materials of the soil disintegrate, and potash in a soluble state is liberated on the layers exposed to the action of the atmosphere. When this has taken place, rich crops of wheat may be again expected.

The alkaline phosphates, as well as the phosphates of magnesia and lime, are necessary for the production of all corn-plants. Now, bones contain the latter, but none of the former salts. These must, therefore, be furnished by means of night-soil, or of urine, a manure which is particularly rich in them.* Wood ashes have been found very useful for wheat in calcareous soils; for these ashes contain both phosphate of lime and silicate of potash. In like manner stable manure and night-soil render clayey soils fertile, by furnishing the magnesia in which they are deficient. 'The ashes of all kinds of herbs and decayed straw are capable of replacing wood ashes.

A compost manure, which is adapted to furnish all the inorganic matters to wheat, oats, and barley, may be made, by mixing equal parts of bone dust and a solution of silicate of potash (known as soluble glass in commerce), allowing this mixture to dry in the air, and then adding 10 or 12 parts of gypsum, with 16 parts of common salt. Such a compost would

* It has been already stated that bran contains phosphate of soda and phosphate of magnesia, so that it is useful as a manure where phosphates are desired. - ED. 
render unnecessary the animal manures, which act by their inorganic ingredients. According to Berthier, 100 parts of the ashes of wheat straw contain, -

Of matter soluble in water
Of matter insoluble in water $\cdot^{\circ} \cdot{ }^{\circ} \quad \begin{array}{r}9 \cdot 0 \\ 91.0\end{array}$

Now 100 parts of the soluble matter contain, -

Carbonic acid . . . . . . a trace

Sulphuric acid . . . . . $\quad .20$

Muriatic acid . . . . $13 \cdot 0$

Silica . . . . . $35 \cdot 0$

Potash and Soda ${ }^{\circ} \cdot{ }^{\circ} \cdot{ }^{\circ} \cdot{ }^{\circ}{ }^{300}$

$100 \cdot 0$

100 parts of the insoluble matter contain, -

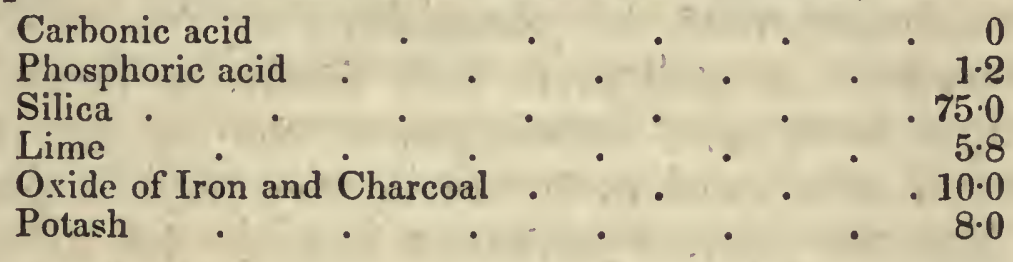

$100 \cdot 0$

The silicate of potash employed in the preparation of the compost described above must not deliquesce on exposure to the air, but must give a gelatinous consistence to the water in which it is dissolved, and dry to a white powder by exposure. It is only attractive of moisture when an excess of potash is present, which is apt to exert an injurious influence upon the tender roots of plants. In those cases where silicate of potash cannot be procured, a sufficiency of wood ashes will supply its place.*

All culinary vegetables, but particularly the cruci-

* In sorne parts of the grand duchy of Hesse, where wood is scarce and dear, it is customary for the common people to club together and build baking ovens, which are heated with straw instead of wood. The ashes of this straw are carefully collected and sold every year at very high prices. The farmers there have found by experience that the ashes of straw form the very best manure for wheat; although it exerts no influence on the growth of fallow-crops (potatoes or the leguminosæ, for example). The stem of whert grown in this way possesses an uncommon strength. The cause of the favorable action of these ashes will be apparent, when it is considered that all corn plants require silicate of potash; and that the ashes of straw consist almost entirely of this compound. - Ev. 
feræ, such as mustard, (sinapis alba and nigra,) contain sulphur in notable quantity. The same is the case with turnips, the different varieties of rape, cabbage, celery, and red clover. These plants thrive best in soils containing sulphates; hence if these salts do not form natural constituents of the soil, they must be introduced as manure. Sulphate of ammonia is the best salt for this purpose. It is most easily procured by the addition of gypsum or sulphate of iron* (green vitriol) to putrefied urine.

Horn, wool, and hoofs of cattle, contain sulphur as a constituent, so that they will be found a valuable manure when administered with soluble phosphates, (with urine, for example.)

Phosphate of magnesia and ammonia forms the principal inorganic constituent of the potato; salts of potash also exist in it, but in very limited quantity. Now the soil is rendered unfitted for its cultivation, even though the herb be returned to it after the removal of the crop, unless some means are adopted to replace the phosphate of magnesia removed in the bulbous roots. This is best effected by mixtures of night-soil with bran, magnesian limestone, or the ashes of certain kinds of coal. I applied to a field of potatoes manure, consisting of night-soil and sulphate of magnesia (Epsom salts), and obtained a remarkably large crop. The manure was prepared by adding a quantity of sulphate of magnesia to a mixture of urine and fæces, and mixing the whole with the ashes of coal or vegetable mould, till it acquired the consistence of a thick paste, which was thus dried by exposure to the sun.

It has been formerly mentioned, that the secondary and tertiary limestones contain potash : marl, and the calcareous minerals used for the preparation of hydraulic mortar, may be particularly specified.

* If sulphate of iron be employed, it ought not to be added in great excess, and the urine must be exposed to the air for some time after, for the purpose of converting the iron into the peroxide. A salt of the protoxide of iron is injurious to vegetation. - ED. 
These have been found to form excellent manures for heavy clayey soils, particularly for such as disintegrate with difficulty. They are most efficacious when burnt, but can only be applied in this state after harvest, and ought to be ploughed into the soil as quickly as possible. By the action of lime upon clay, the potash contained in the latter is rendered soluble. This may easily be shown by mixing one part of marl with half its weight of burned lime, adding water, and setting aside the mixture to repose for some time. Even after a space of 24 hours, an appreciable quantity of potash may be detected in the water.**

A most striking proof of the influence of potash upon vegetation has been furnished by the investigations of the "administration" of tobacco in Paris. For many years accurate analyses of the ashes of various sorts of tobacco have been executed, by the orders of the "administration"; and it has been found, as the result of these, that the value of the tobacco stands in a certain relation to the quantity of potash contained in the ashes. By this means a mode was furnished of distinguishing the different soils upon which the tobacco under examination had been cultivated, as well as the peculiar class to which it belonged. Another striking fact was also disclosed through these analyses. Certain celebrated kinds of American tobacco were found gradually to yield a smaller quantity of ashes, and their value diminished in the same proportion. For this information I am indebted to M. Pelouze, professor of the Polytechnic School in Paris.

* One of the causes of the advantages produced by subsoil ploughing is, that it exposes the soil to the disintegrating influences of the atmosphere. Hence it is that the subsoil plough is so beneficial in siliceous soils, and exerts no apparent effect upon those which contain much clay. The former disintegrate and liberate their potash both with facility and rapidity; whilst the disintegration of the latter proceeds with slowness, and no appreciable effects are produced. (See Journal of the Agricultural Society, Vol. II. p. 27.) It is probable, however, that if the land received a dressing of lime after subsoil ploughing, the effects would be produced more rapidly. - ED. 
There are certain plants which contain either no potash, or mere traces of it. Such are the poppy, (papaver somniferum,) which generates in its organism a vegetable alkaloid; Indian corn (zea mays); and helianthus tuberosus. For plants such as these the potash in the soil is of no use, and farmers are well aware that they can be cultivated without rotation on the same soil, particularly when the herbs and straw, or their ashes, are returned to the soil after the reaping of the crop.

One cause of the favorable action of the nitrates of soda and potash must doubtless be, that through their agency the akalies which are deficient in a soil are furnished to it. Thus it has been found that in soils deficient in potash, the nitrates of soda or potash have been very advantageous; whilst those, on the other hand, which contain a sufficiency of alkalies, have experienced no beneficial effects through their means. In the application of manures to soils we should be guided by the general composition of the ashes of plants, whilst the manure applied to a particular plant ought to be selected with reference to the substances which it demands for its nourishment. In general, a manure should contain a large quantity of alkaline salts, a considerable proportion of phosphate of magnesia, and a smaller proportion of phosphate of lime; azotized manure and ammoniacal salts cannot be too frequently employed.

In the following part of this chapter I shall describe a number of analyses of soils executed by Sprengel, together with observations on their sterility and fertility, as stated by that distinguished agriculturist. It is unnecessary to describe the modus operandi used in the analyses of these soils, for this kind of research will never be made by farmers, who must apply to the professional chemist, if they wish for information regarding the composition of their soils.

Under the term surface-soil; we mean that portion of soil which is on the surface; whilst by subsoil we 
mean that which is below the former, and out of reach of the ordinary plough.

CHEMICAL COMPOSITION OF CERTAIN SOILS, ACCORDING TO ANALYSIS.

1. Surface-soil (A) a good loamy soil, from the vicinity of Gandersheim. It is remarkable for producing uncommonly fine red clover when manured with gypsum. (B) is an analysis of the subsoil. 100 parts contain :-

\begin{tabular}{|c|c|c|}
\hline ilica, with tne siliceous sand & $91 \cdot 331$ & $93 \cdot 883$ \\
\hline Alumina $\therefore \quad \cdot \quad \cdot$ & $1 \cdot 344$ & $1 \cdot 944$ \\
\hline Peroxide of iron, with a little protoxide & 1.562 & $2 \cdot 226$ \\
\hline $\begin{array}{l}\text { Peroxide of manganese } \\
\text { Magnesia and silica, in combination with }\end{array}$ & 0.082 & $0 \cdot 320$ \\
\hline $\begin{array}{l}\text { Magnesia and silica, in combination with } \\
\text { sulphuric acid and humus . } \\
\text { Magnesia, with silica and humic acid }\end{array}$ & 0.80 & 0 \\
\hline $\begin{array}{l}\text { Magnesia, with silica and humic acid } \\
\text { combined }\end{array}$ & $0 \cdot 440$ & 10 \\
\hline $\begin{array}{l}\text { Potash, in combination with silica } \\
\text { Soda, principally in combination with }\end{array}$ & & \\
\hline silica, and a little as common salt & & 0.06 \\
\hline Phosphoric acid $\cdot \quad \cdot \ldots \quad \therefore \quad \cdot \quad$ : & 0.0 & $0 \cdot 190$ \\
\hline Sulphuric acid in combination with lime & & 012 \\
\hline Chlorine (in common salt) ${ }^{\cdot} \cdot \dot{ }$ & 012 & 012 \\
\hline Humus, with traces of azotized matter & & \\
\hline & & \\
\hline
\end{tabular}

An inspection of the abore analyses will show that the soil contains a very small proportion of salts of sulphuric acid, - a circumstance which accounts for the favorable action of gypsum upon it.

2. The surface-soil (A) is a fine-grained loamy soil from Gandersheim, distinguished for the remarkably large crops of beans, peas, tares, \&c., which it produces when manured with gypsum. (B) is the analysis of the subsoil. 100 parts contain :-

(A)

Silica, with fine siliceous sand

Alumina

Peroxide and protoxide of iron

Peroxide of manganese

Lime, principally combined with phosphoric acid and humus
$90 \cdot 221$

$2 \cdot 106$

3.951

0.960
(B)

$92 \cdot 324$

2.262

2.914

2.960 
Magnesia, with silicate of potash, \&c. . $0 \cdot 7 \cdot 30$

(B)

$0 \cdot 340$

$0 \cdot 304$

Potash

0.066

Soda

0.010

$0 \cdot 367$

a trace

$0 \cdot 122$

Sulphuric acid (in gypsum) . . . a trace

0.010

$0 \cdot 100$

0.004

Chlorine (in common salt)

. 0.900

$0 \cdot 140$

0.228

Loss

$100 \cdot 000$

$100 \cdot 000$

The analysis of this soil shows, that, with the exception of gypsum, every ingredient is present which is requisite for the nourishment of leguminous plants. Hence it is that gypsum exerts such a favorable influence upon it.

3. Surface-soil (A) a strong loamy sand, from Brunswick. (B) the analysis of the subsoil. 100 parts contain :-

Silica, with coarse siliceous sand (A)

Alumina . . 0.504

Peroxide and protoxide of iron . . 24496

Peroxide of manganese . . a trace

(B)

Lime

$0.038-0.019$

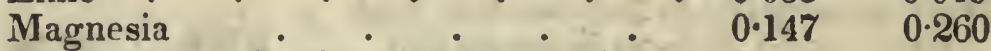

Potash and soda, the greatest part in

combination with silica ? $\quad \cdot \quad 0.090 \quad 0.079$

$\begin{array}{llllll}\text { Phosphate of iron } \quad . \quad & 0.164 & 0.110\end{array}$

Sulphuric acid (in gypsum) . $\quad . \quad 0.007$ a trace

Chlorine (in common salt) $\quad \cdot \quad 0.010$ a trace

Humus

- $0.846 \quad 0.226$

$100 \cdot 000 \quad 100 \cdot 000$

This soil was much improved by manuring with lime and ashes. It was then found well fitted for clover, beans, and peas.

4. Surface-soil (A) a loamy sand, from the environs of Brunswick. (B) analysis of the subsoil at the depth of 3 feet. 100 parts contain :-

$\begin{array}{lcc} & (\text { A) } & (\text { B) } \\ \text { Silica and fine siliceous sand . } \quad . \quad 94 \cdot 724 & 97 \cdot 340 \\ \text { Alumina } & . \quad 1 \cdot 638 & 0.806\end{array}$

Protoxide and peroxide of iron with man-

$\begin{array}{lllllll}\text { ganese . } & . & . & 1.960 & 1.201\end{array}$

Lime . . . . . . . $1.028 \quad 0.296$

Magnesia . . . . a trace 0.095

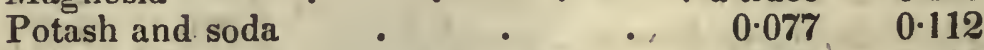

Phosphoric acid . . . . $0.024 \quad 0.015$

Gypsum . $\quad . \quad$. $\quad 0.010$ a trace 


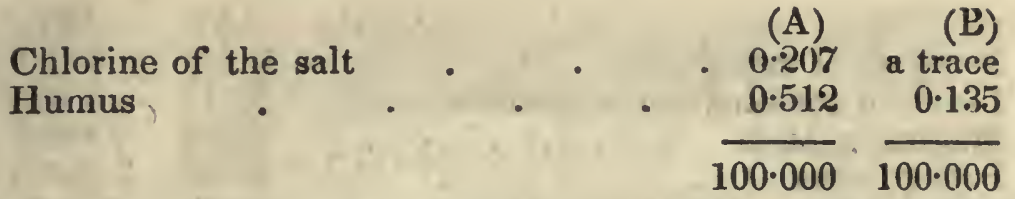

This soil produces luxuriant crops of lucern and sainfoin, as well as of all other plants the roots of which penetrate deeply into the ground. The reason is apparent. 'The subsoil contains magnesia, which is wanting in the surface-soil.

5. Surface-soil (A) a loamy sand, from the environs of Brunswick. (B) analysis of the subsoil at a depth of 2 feet. 100 parts contain :-

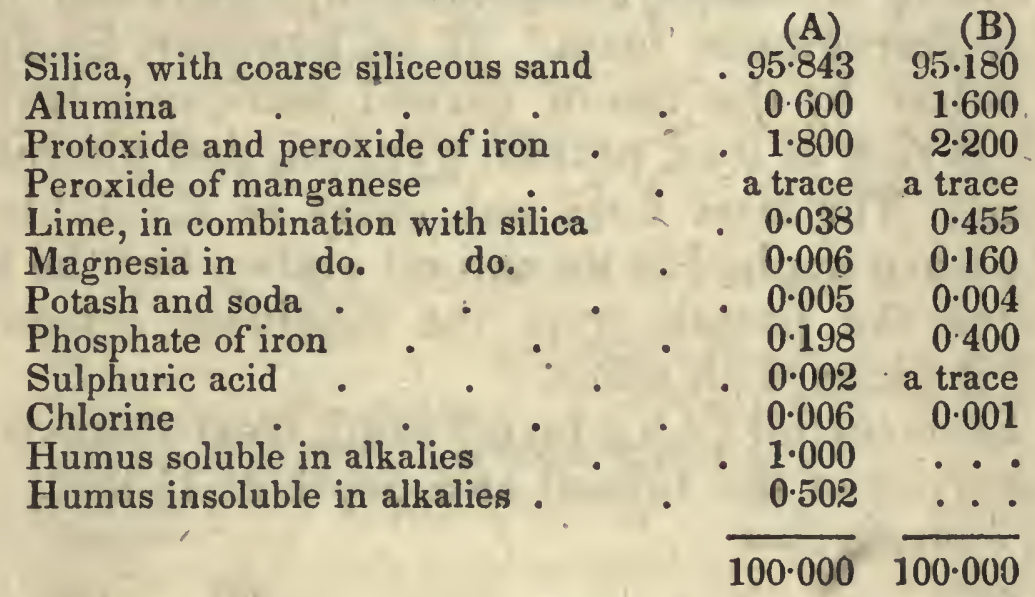

This soil is characterized by its great sterility. White clover could not be made to grow upon it. The obvious cause of its poverty is a deficiency of lime, magnesia, potash, and gypsum; for we find that the fertility of the soil was much increased by manuring it with marl. The white clover, which formerly had refused to grow on this soil, now grew upon it with much luxuriance. The aridity of the soil could not have been the cause of its sterility, for the stiff nature of the subsoil on which it rested prevented a deficiency of moisture.

6. Surface-soil (A) a loamy land from the environs of Brunswick. (B) the analysis of the subsoil, at a depth of 2 feet. 100 parts contain:-

Silica, with fine siliceous sand . $\quad .94 \cdot 998$

Alumina

Protoxide and peroxide of iron

- $1 \cdot 080$ 


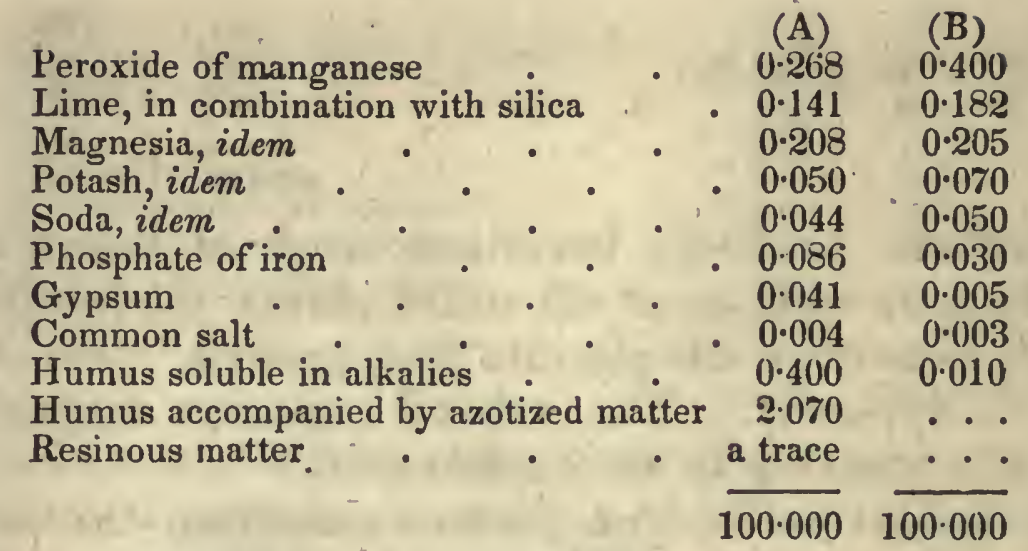

This soil is by no means remarkable for its sterility, but is decidedly improved by manuring with burned ferruginous loam. It is, however, rendered still better by the use of burned marl, - a manure which is rich in iron, potash, gypsum, and phosphate of lime. The marl does not exert so favorable an action when applied in its natural state; but the heat liberates the potash from the insoluble compound which it forms with silica:

7. Surface-soil (A) a loamy sand, from Brunswick. (B) analysis of the subsoil at a depth of $1 \frac{1}{2}$ feet. 100 parts contain :-

\begin{tabular}{|c|c|}
\hline Silica, with fine siliceous sand & 92.980 \\
\hline Alumina & 0.820 \\
\hline $\begin{array}{l}\text { Protoxide and peroxide of iron } \\
\text { Peroxide of manganese }\end{array}$ & $\begin{array}{l}1 \cdot 666 \\
0 \cdot 188\end{array}$ \\
\hline Lime, combined with silica & 0.748 \\
\hline Magnesia, idem & \\
\hline $\begin{array}{l}\text { Potash, idem } \\
\text { Poda idem }\end{array}$ & $\begin{array}{l}0.065 \\
0.130\end{array}$ \\
\hline $\begin{array}{l}\text { Soda, idem } \\
\text { Phosphate of iron }\end{array}$ & 0.246 \\
\hline Sulphuric acid contained in gypsum & \\
\hline Chlorine & \\
\hline $\begin{array}{l}\text { Humus soluble in alkalies } \\
\text { Humus, with azotized orranic remains }\end{array}$ & $\begin{array}{l}0.764 \\
2.225\end{array}$ \\
\hline & \\
\hline
\end{tabular}

This soil when manured with gypsum is very favorable to the production of leguminous plants and red clover. But it is very remarkable, on account of the rust which always attacks the corn plints which may be grown upon it. This rust and mildew (uredo linearis, puccinia graminis) is a disease which at- 
tacks the stem and leaves, and is quite different from the brand (uredo glumarum) which appears on the seeds and organs of reproduction. Rust is most frequently detected on plants growing on soils which contain bog-ore, or turf iron-ore. According to Sprengel, rust contains phosphate of iron, to which this chemist ascribes the origin of the disease. It is very possible that other causes may operate in the production of similar diseases.

8. Soil, a fine-grained loamy marl, from the vicinity of Schoningen. It produces corn, which is, however, very liable to blight. 100 parts contain:-

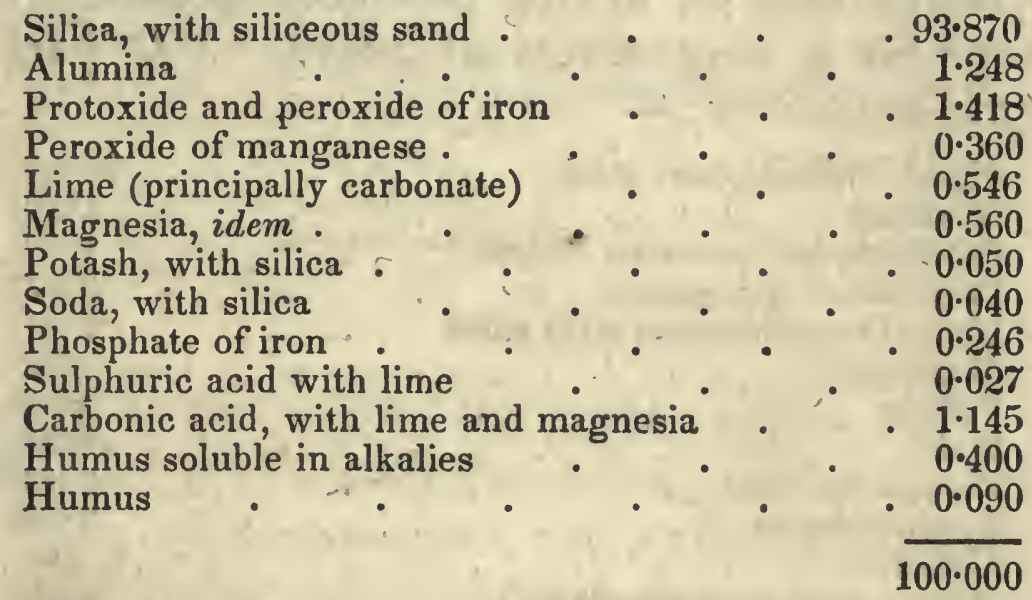

It will be observed that a considerable quantity of phosphate of iron is contained in this soil, and the corn which grows upon it is, as in the former case, disposed to rust.

9. Surface-soil (A) a loamy soil, from Brunswick, remarkable on account of producing buck-wheat, which is exceedingly poor in the grain. (B) analysis of the subsoil at a depth of $1 \frac{1}{2}$ foot. 100 parts contain :

(A)

$.95 \cdot 114$

$1 \cdot 080$

- 1.900

0.320

0.380

$0 \cdot 300$

- 0.020

0.004

0.052

0.006
(B)

$92 \cdot 458$

$2 \cdot 530$

2502

0.920

0.710

0.551

$0 \cdot 120$

0.034

0.175

a trace 


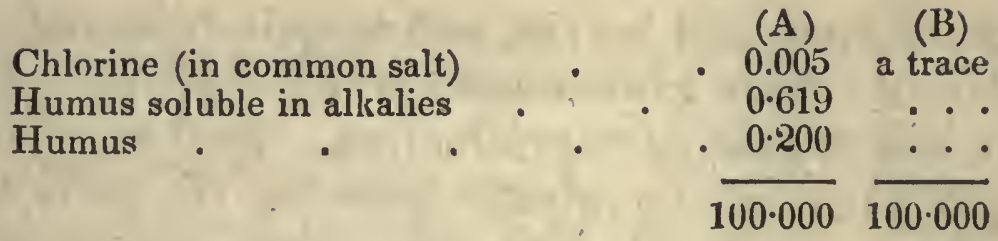

By manuring the land with wood ashes, the soil is enabled to produce buck-wheat, with rich grain; the leguminous plants also thrive luxuriantly upon it. This increased fertility is due to the ashes, by means of which both potash and phosphates are supplied to the land.

10. Subsoil of a loamy, sandy soil, from Brunswick. -It is remarkable for having produced excellent crops of hops for a long series of years. 100 parts, by weight, consist of : -

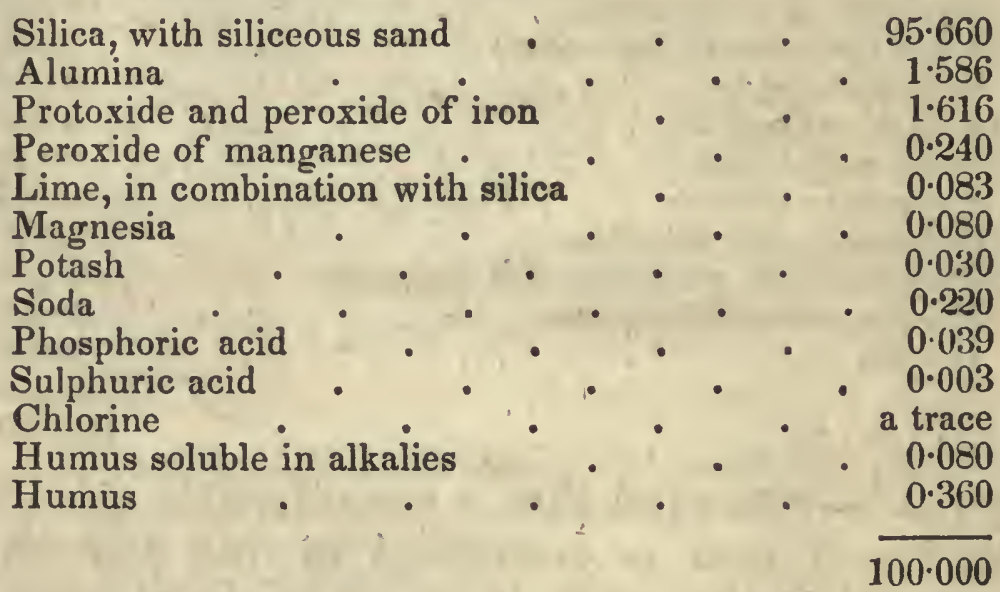

Although the hops contain a large quantity of potash, soda, phosphoric acid, sulphuric acid, lime, and magnesia, yet we do not find that these exist in the soil in superabundant quantity. Nor is it necessary that they should, for the roots of the hops penetrate 8 or 10 feet deep into the soil, and search out the materials fitted to nourish the plants. Hence it is that hops thrive well on soils comparatively poor in their proper ingredients. The same is the case with all plants of a similar nature, the roots of which possess a tendency to extend in search of food; we see this particularly in lucern and sainfoin. 


\section{SOILS OF HEATHS.}

11. Soil of a heath converted into arable land, in the vicinity of Brunswick. It is naturally sterile, but produces good crops when manured with lime, marl, cow-dung, or the ashes of the heaths which grow upon it.

Silica, and coarse siliceous sand $\quad$. $\quad 71.504$

Alumina . . 0 0.780

Protoxide and peroxide of iron, principally com: bined with humus

Peroxide of manganese, idem . . . 0.220

Lime, idem . . . . . $0 \cdot 134$

Magnesia, idem . . . 0.032

Potash and soda principally as silicates $\quad 0.058$

Phosphoric acid, (principally as phosphate of iron) $\quad 0.115$

Sulphuric acid (in gypsum) . . . 0.018

Chlorine (in common salt) . . . 0.014

Humus soluble in alkalies . . . . 9.820

Humus, with vegetable remains $\quad$ - 14.975

Resinous matters $\quad$. $\quad$. 1.910

$100 \cdot 000$

Ashes of the soil of the heath, before being converted into arable land:-

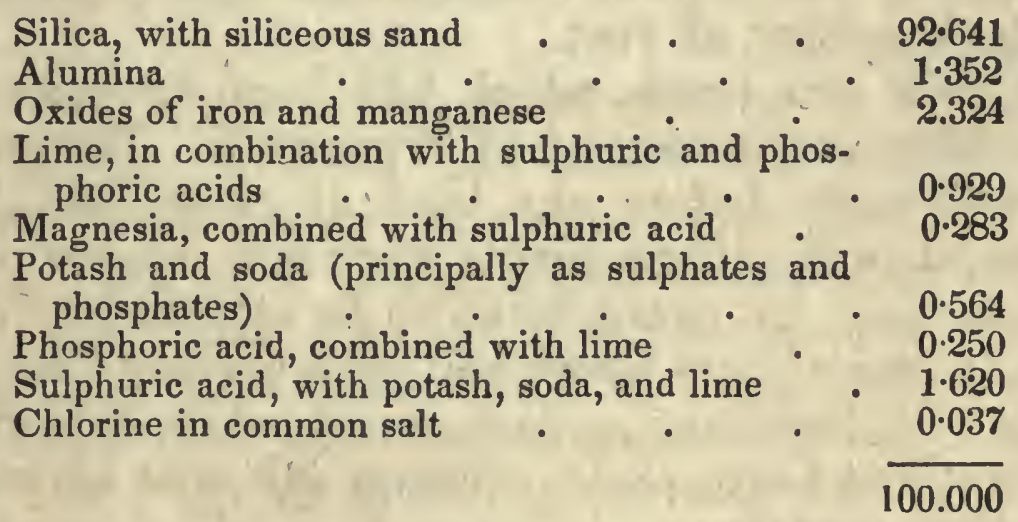

12. Surface-soil of a fine-grained loam, from the vicinity of Brunswick. It is remarkable from the circumstance, that not a single year passes in which corn plants are cultivated upon it without the stem of the plants being attacked by rust. Even the grain is covered with a yellow rust, and is much shrunk. 100 parts of the soil contain:-

Silica and fine siliceous sand 
Protoxide and peroxide of manganese . 0.840

Lime principally combined with silica . $\quad$. 1.459

Magnesia idem" . . . . 0.280

Potash and soda idem . $\quad . \quad$. $\quad .0 .090$

Phosphoric acid in combination with iron . 0.505

Sulphuric acid in combination with lime . 0.068

Chlorine in common salt . . . . 0.006

Humus . $\quad . \quad$. $\quad$. $\quad$. $\quad$. $1 \cdot 109$

$100 \cdot 000$

'This soil does not suffer from want of drainage: it is well exposed to the sun, is in an elevated situation, and in a good state of cultivation. In order to ascertain whether the rust was due to the constituents of the soil, (phosphate of iron?) or to certain fortuitous circumstances unconnected with their operation, a portion of the land was removed to another locality, and made into an artificial soil of fifteen inches in depth. Upon this barley and wheat were sown; but it was found, as in the former case; that the plants were attacked by rust, whilst barley growing on the land surrounding this soil was not at all affected by the disease. From this experiment it follows, that certain constituents in the soil favor the development of rust.

13. Soil of a heath, which had been brought into cultivation in the vicinity of Brunswick. The analysis was made before any kind of crops had been grown upon it. Corn-plants were first reared upon the new soil, but were found to be attacked by rust, even on those parts which had been manured respectively with lime, marl, potash, wood ashes, bone-dust, ashes of the heath plant, common salt, and ammonia. 100 parts contain :-

Silica with coarse siliceous sand

Alumina

51.337

Protoxide and peroxide of iron in combination with phosphoric and humic acids . . 0.398

Protoxide and peroxide of manganese . . 0.005

Lime in combination with humus . . 0.230

Magnesia idem . . . . . . 0.040

Potash and soda . . . . 0.010

Phosphoric acid . . . . . 0.066

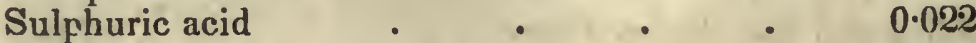

Chlorine 
Resinous matters

Coal of humus and water

$100 \cdot 000$

The next analysis represents the soil after being burnt. 100 parts by weight of the soil left after ignition only 50 parts. 100 parts of these ashes consisted of : -

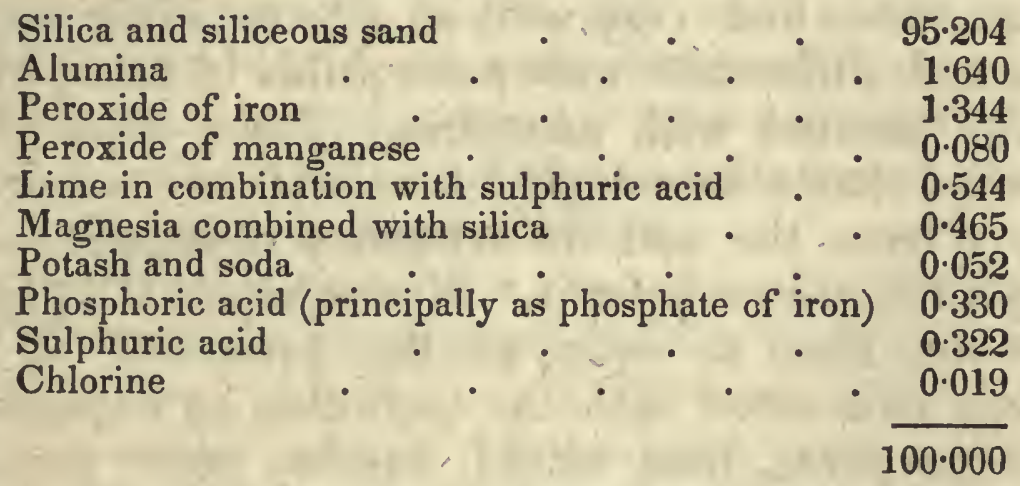

By comparing this analysis with the one which has preceded it, an increase in certain of the constituents is observed, particularly with respect to the sulphuric acid, potash, soda, magnesia, oxide of iron, oxide of manganese, and alumina. From this it follows, that the humus, or in other words, the vegetable remains, must have contained a quantity of these substances confined within it, in such a manner that they were not exhibited by analysis.

Oats and barley were sown on this land the second year after being reclaimed, and both suffered much from rust, although different parts of the soil were manured with marl, lime, and peat-ashes; whilst other portions were left without manure. In the first year, all the different parts of the field produced potatoes, but they succeeded best in those divisions which had been manured with peat-ashes, lime and marl. In the second year, oats mixed with a little barley were sown upon the soil; and the straw was found to be strongest on the parts treated. with peat-ashes, lime, marl, and ashes of wood. Red clover was sown on the third year; it appeared in best condition on those portions of the soil manured with marl and lime. Upon the divisions of the field 
which had been left without manure, as well as on those manured with bone-dust, potash, ammonia, and common salt, the clover scarcely appeared above ground. The divisions of the field, which had been manured in the first year with peat-ashes, ammonia, and ashes of wood, were sown with buckwheat after the removal of the first crop of clover. The buckwheat succeeded very well on all the divisions, yet a marked difference was perceptible in favor of the portion treated with ammonia. These experiments show us, that a dressing of lime did not completely remove from the soil its tendency to impart rust to the plants grown upon it. Nevertheless it is highly probable, that as soon as the protoxide of iron became converted into the peroxide by exposure to the atmosphere, lime would possess more power in decomposing the phosphate of iron.

14. Subsoil of a loamy soil in the vicinity of Brunswick. It is remarkable from the circumstance that sainfoin cannot be cultivated upon it more than two or three years in succession. The portion analyzed was taken from a depth of five feet. 100 parts contained :-

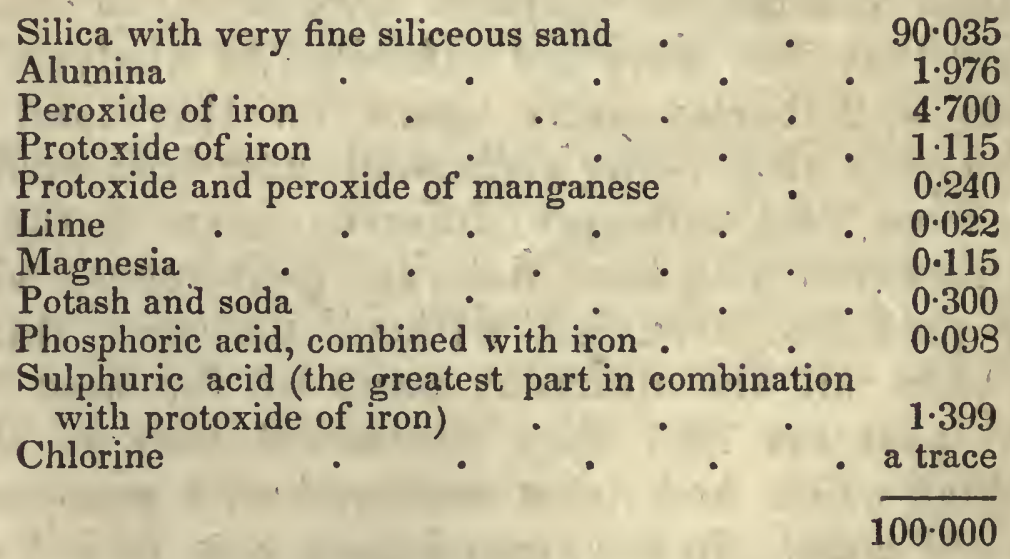

Now the results of the analysis give a sufficient account of the failure of the sainfoin. The soil contains above one per cent. of sulphate of the protoxide of iron (green vitriol of commerce), a salt which exerts a poisonous action upon plants. Lime is not present in quantity sufficient to decompose 
this salt. Hence it is that sainfoin will not thrive on this soil, nor indeed lucern, or any other of the plants with deep roots. The evil cannot be obviated by any methods sufficiently economical for the farmer, because the soil cannot be mixed with lime at a depth of five or six feet. For many years experiments have been made in vain, in order to adapt this soil for sainfoin and lucern, and much expense incurred, which could all have been saved, had the soil been previously analyzed. This example affords a most convincing proof of the importance of chemical knowledge to an agriculturist.

15. Surface-soil (A) of a sandy loam in the vicinity of Brunswick, celebrated for its beautiful crops of clover, rye, potatoes, and barley. The clover must, however, always be manured with gypsum. (B) is an analysis of the subsoil at the depth of $1 \frac{1}{2}$ foot. 100 parts contain :-

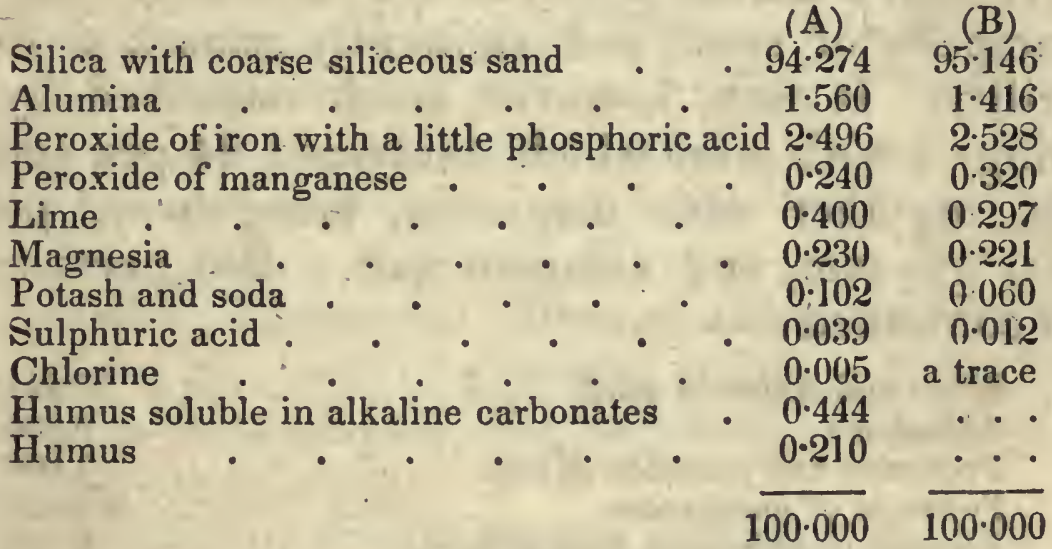

The best property of this soil is, that its inferior layers are nearly of the same composition as the superior, as far as the inorganic constituents are concerned. It is a soil upon which the plants mentioned above will seldom fail; and as it possesses a very good mixture to the depth of four or five feet, it would, doubtless, produce lucern also.

16. Surface-soil (A) of a sandy loam in the vicinity of Brunswick. It produces excellent crops of oats and clover, when the latter is manured with gypsum. 
(B) Analysis of the subsoil taken from a depth of $1 \frac{1}{2}$ foot. 100 parts contain :-

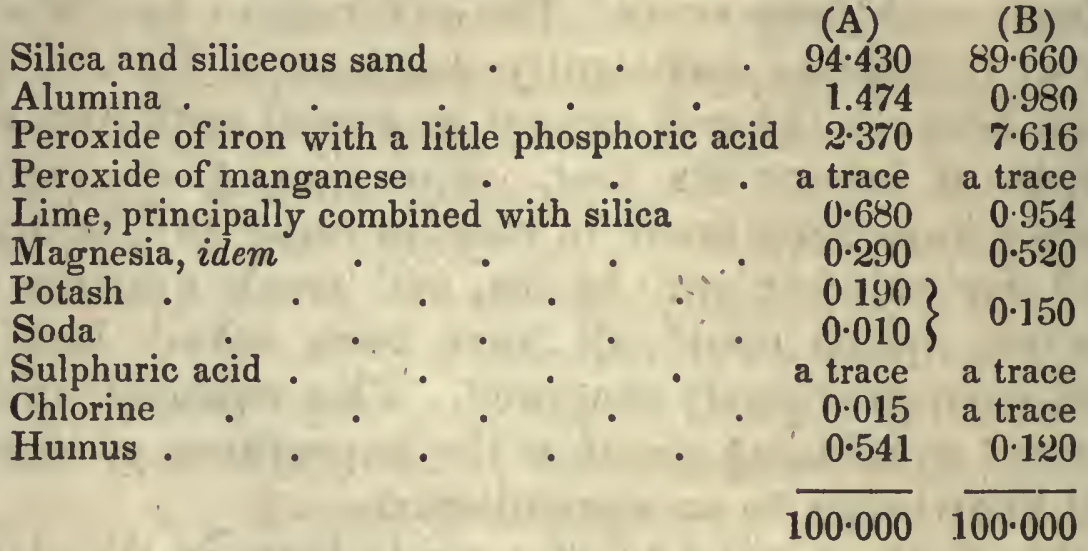

Both the surface and the sub-soil contain only traces of sulphuric acid. Hence the application of gypsum is attended with great benefit. Without doubt, marl and lime will be found of essential service.

17. Soil from the environs of Brunswick, consisting principally of sand, and eminently remarked for its sterility. It was, however, much improved by manuring it with marl which contained 24 per cent. of lime, together with magnesia, manganese, potash, soda, gypsum, and common salt. 100 parts of the soil contained :-

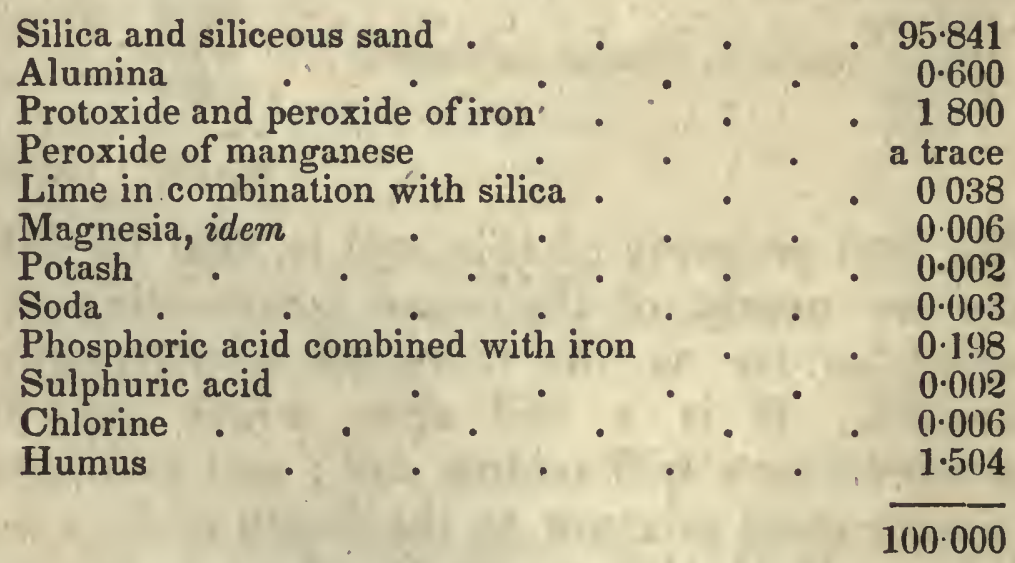

Here another proof is presented, that a soil may be very rich in humus and yet be very poor as regards fertility. By means of the marl, the inorganic ingredients of the plants are furnished to the soil, which contains them in very small quantity. 
18. The soil of a very fertile loam from the vicinity of Walkenried. 100 parts contain:-

Silica, with coarse-grained siliceous sand _ $\quad 88.456$

Alumina . . . 0.650

Peroxide and protoxide of iron, accompanied by much magnetic iron sand . . . . 5608

'Peroxide of manganese . $\quad . \quad \ldots \quad$. $\quad 0.560$

Carbonate of lime _ $\quad$. $\quad$. $\quad$. . . 1.063

$\begin{array}{lllll}\text { Carbonate of magnesia } & \therefore & 0 & 0 & 1.688\end{array}$

Potash combined with silica $\quad$. . . 0.040

Soda combined with silica . . . . 0.012

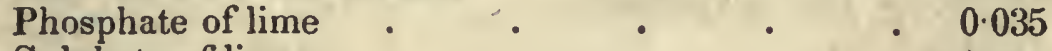

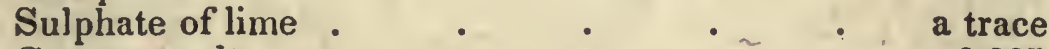

Common salt $\quad . \quad . \quad \% \quad 0005$

Humus soluble in alkalies _. . . . 0.550

Humus with several azotized organic remains . 1.333

$100 \cdot 000$

Gypsum acts most excellently upon this land. The soils in the southern range of the Harz mountains are particularly remarked for containing more magnesia, than lime. Even the different varieties of marl contain a considerable quantity of magnesia. Thus in a specimen of marl obtained from the vicinity of Walkenried, I obtained $55 \frac{1}{2}$ per cent. carbonate of lime, and $30 \frac{1}{2}$ per cent. carbonate of magnesia; in another 41 per cent. lime, and 11 per cent. magnesia; and in a third, $47 \frac{1}{2}$ per cent. lime, and $13 \frac{1}{2}$ per cent. magnesia. Most of these soils contain also $\frac{1}{2},-1$ per cent. of gypsum, and $\frac{1}{2},-1$ per cent. phosphate of lime, and are, therefore, well fitted for manuring other lands.

19. Subsoil of a loam from a depth of $1 \frac{1}{2}$ foot. It occurs in the vicinity of Brunswick. The surfacesoil is remarkable on account of producing beautiful' red clover on being manured with gypsum; although the soil itself contains only traces of lime, magnesia, potash, and phosphoric acid., 100 parts of the subsoil contained :

Silica and coarse siliceous sand.

Alumina

Protoxide and peroxide of iron

Peroxide of manganese

Carbonate of lime

Carbonate of magnesia

Potash and soda

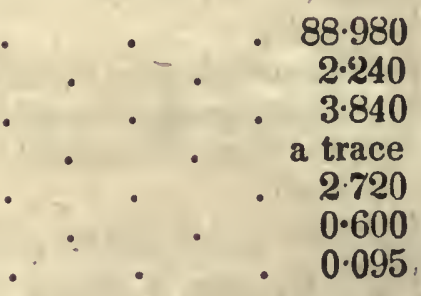




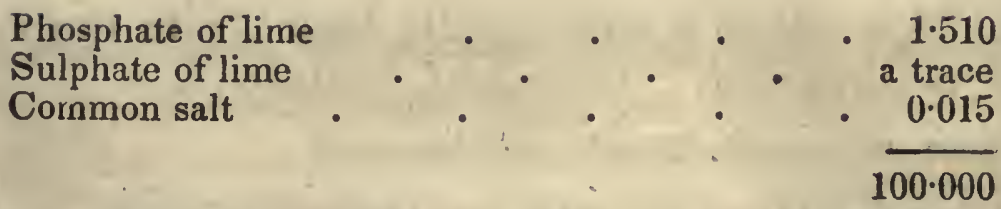

At a greater depth than the subsoil of which the analysis is here given, the soil passes into marl, which contains $20 \frac{1}{2}$ per cent. of carbonate of lime. The sulphuric acid deficient in the soil was supplied by means of the gypsum.

\section{SOILS IN THE KINGDOM OF HANOVER.}

20. (A) Analysis of a barren heath-soil from Aurich in Ostfriesland; (B) a sandy soil containing much humus but also sterile; (C) a sandy soil possessing the same characters as B. 100 parts contained :-

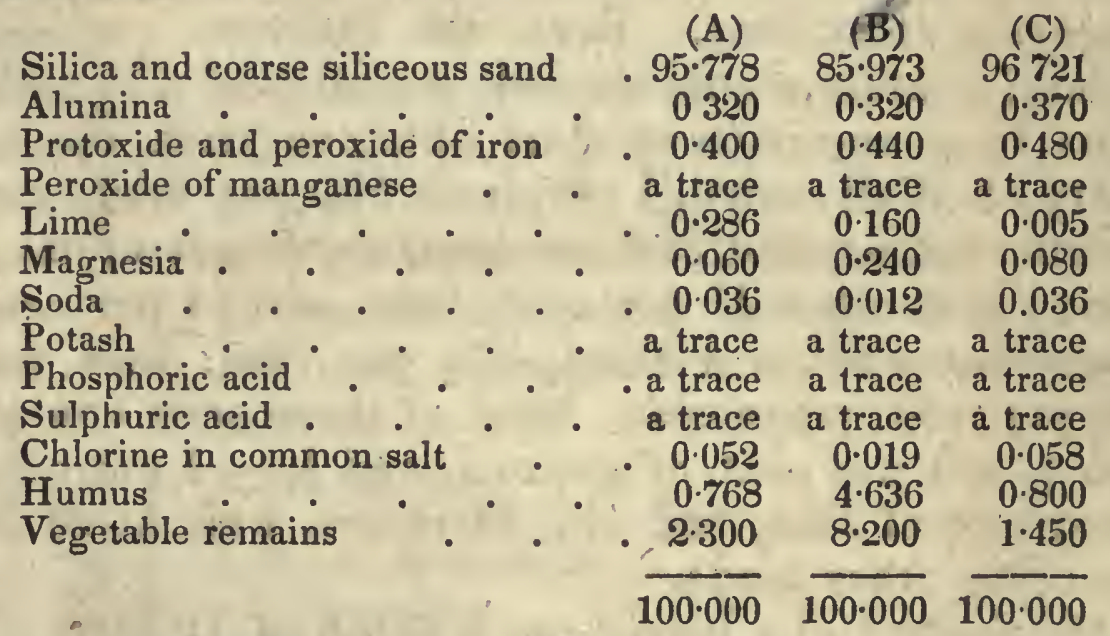

21. Analysis of the clayey subsoil of a moor, which, after being burned, is used as a manure to the above soils A, B, C. 100 parts contain :-

Silica and siliceous sand _ . . $87 \cdot 219$

Alumina . . . . . 4.200

Peroxide of iron with a little phosphoric acid . $\quad 5.200$

Peroxide of manganese $\quad$ • . 0.310

Lime . . . . . . . 0.320

Magnesia . . . . . 0.380

Potash principally combined with silica . 0.130

Soda principally combined with silica . . 0 $0 \cdot 274$

Sulphuric acid combined with lime, magnesia, and potash

Chlorine

0.965

0.002

Humus

$1 \cdot 000$ 
By comparing this analysis with that of the three soils which have preceded, it will be observed that this subsoil is fitted to impart to them those mineral ingredients in which they are deficient.

22. Surface soil of a barren heath in the vicinity of Walsrode in Luneberg. 100 parts by weight contain :-

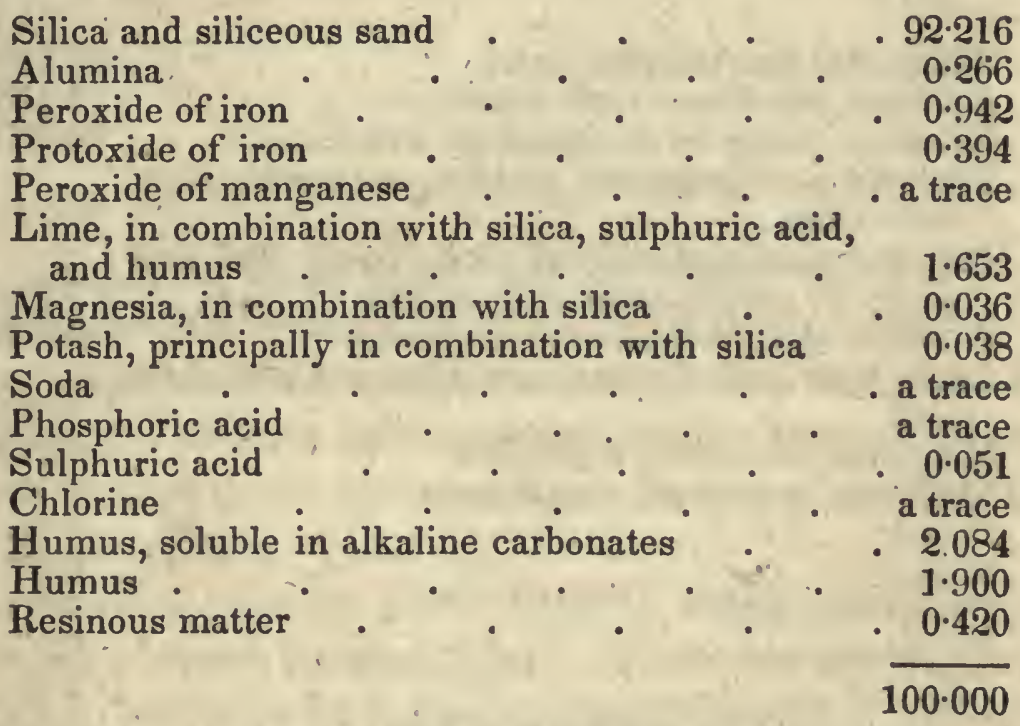

This soil contains a large quantity of protoxide of iron, which, together with a deficiency of phosphoric acid, is the cause of its sterility. But when this land was manured with the ashes of peat, it was rendered much more fertile. The ashes used for this purpose were found to contain in 100 parts :-

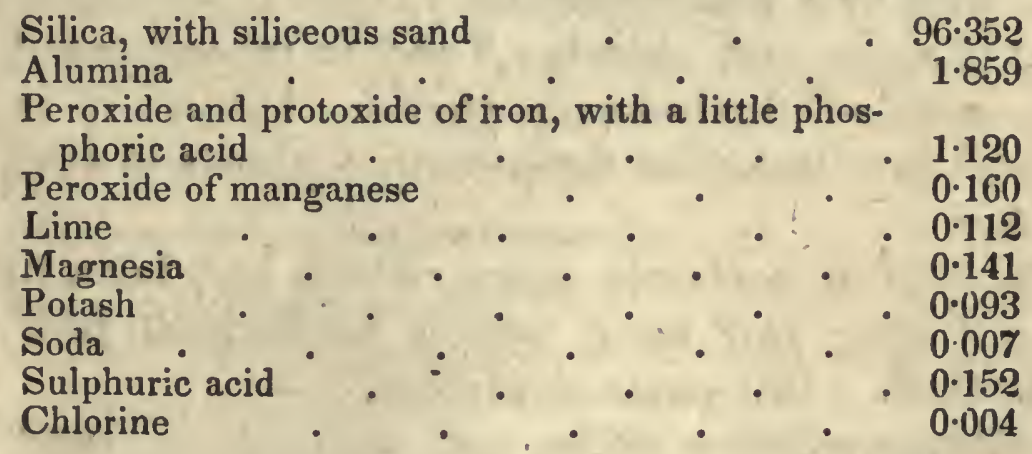

$100 \cdot 000$

The ashes, on exposure to the air, absorbed ammonia.

23. Analysis of a very fertile loamy soil from Göttingen. It is very rich in humus, and produces beau- 
tiful crops of peas, beans, lucern, and beet. The sieve separates from 100 parts of the soil :-

Small stones, principally limestone $\quad \therefore \quad$ - 1

Quartzy sand, with a little magnetic iron sand . I 15

Earthy part . . . . . . 84

100

100 parts of the soil, freed from stones, consists of :

Silica, and fine siliceous sand . . . . . 83298

Alumina, combined with silica $\quad . \quad 1.413$

Alumina, partly in combination with humus . 3.715

Peroxide and protoxide of iron, in combination with silica

Peroxide and protoxide of iron, partly free and partly in combination with humus . 2244

Peroxide and protoxide of manganese $\quad 0.280$

Lime, with coal of humus, sulphur, and phosphoric acid

1.814

Magnesia, combined with silica . . 0.422

Magnesia, combined with humus . $\quad . \quad .0 .400$

Potash : . . . . . . 0.003

Soda

Phosphoric acid

Sulphuric acid

Chlorine $: 0.002$

Carbonic acid (as carbonate of lime) _ $\quad 0.440$

Humus, soluble in alkalies . . . . 0.789

Humus, with a little water $\quad . \quad 3 \cdot 250$

Nitrogenous matter . . . . . 0.960

Resinous matter . . . . . a trace

$100 \cdot 000$

The subsoil is of the same composition as the surface, with this difference only, that it contains more potash, soda, and chlorine, * and is interspersed with fragments of fresh-water shells. Hence it is that the soil produces the deep-rooted plants in such luxuriance.

24. Soil of a sterile moor, which had been burned three times, and upon which buckwheat had been cultivated. 100 parts contained :-

Humus, soluble in alkalies $\quad$ • $\quad 9 \cdot 250$

Vegetable remains, charcoal, quartzy sand, and earthy particles

$100 \cdot 000$

* The portion of the surface-soil subjected to analysis was taken from the field after long-cortinued rain. Hence the small quantity of salts of potash and soda. 
100 parts by weight left, after ignition, 10 parts of ashes. 100 parts of these ashes consisted of :-

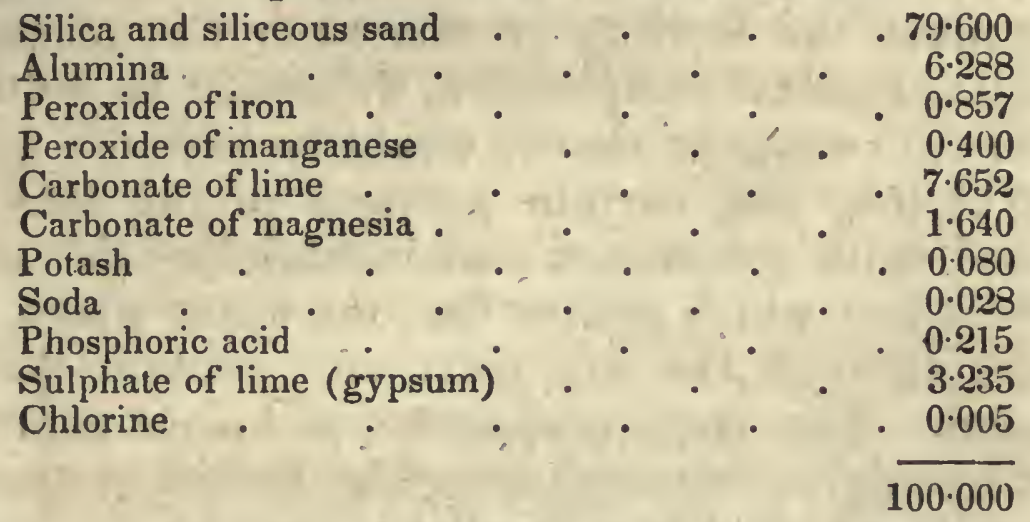

Soils such as this, after having been burned several times, and made to produce buckwheat, are completely deprived of their potash and soda; and in consequence of this are rendered quite barren. Hence it is that ashes of wood exert such an astonishing effect upon them.

25. Analysis of a very fertile loamy sand, from Osnabrück, near Rotherfeld. It is remarkable for being manured only once every 10 or 12 years, and bears beautiful wheat as the last crop. 100 parts contain :-

Silica, with coarse siliceous sand .

Alumina

. $86 \cdot 200$

Peroxide and protoxide of iron, with a little phosphoric acid . . . . 2.900

Peroxide of manganese . . . . $0 \cdot 100$

Carbonic acid, and a little phosphate of lime . $\quad 4 \cdot 160$

Carbonate of magnesia . . . . . 0.520

Potash and soda $\quad . \quad \ldots \quad . \quad . \quad 0.035$

Phosphoric acid . . . . . . 0 0 020

Sulphuric acid . . . . . . . 0.021

Chlorine . . . . . . 0.010

Humus, soluble in alkaline carbonates _ 0544

Humus $\quad . \quad . \quad . \quad \therefore 370$

Nitrogenous matter $\quad$. $\quad . \quad$. $\quad . \quad 0 \cdot 120$

$100 \cdot 000$

The soil in question lies on the southern exposure of a hill, which consists of layers of limestone and marl. The rain-water penetrates through these layers, and becomes saturated with the soluble salts contained in them, such as potash, gypsum, common salt, lime, magnesia, and saltpetre. It afterwards 
reaches the soil, and manures it with these ingredients. It is only in this manner that we are enabled to explain the fertility of this soil ; for, reasoning from its chemical composition, we should be induced, $\dot{a}$ priori, to suppose that it would be barren. At the base of this hill, certain portions of the land are covered with calcareous tuff, containing the above salts : a fact which proves that the water which penetrates through the soil must also contain them in solution. The large proportion of humus exhibited by the analysis depends upon the nature of the manure with which it was treated.

26. Analysis of a heavy alluvial soil, from Norden. 100 parts contain :-

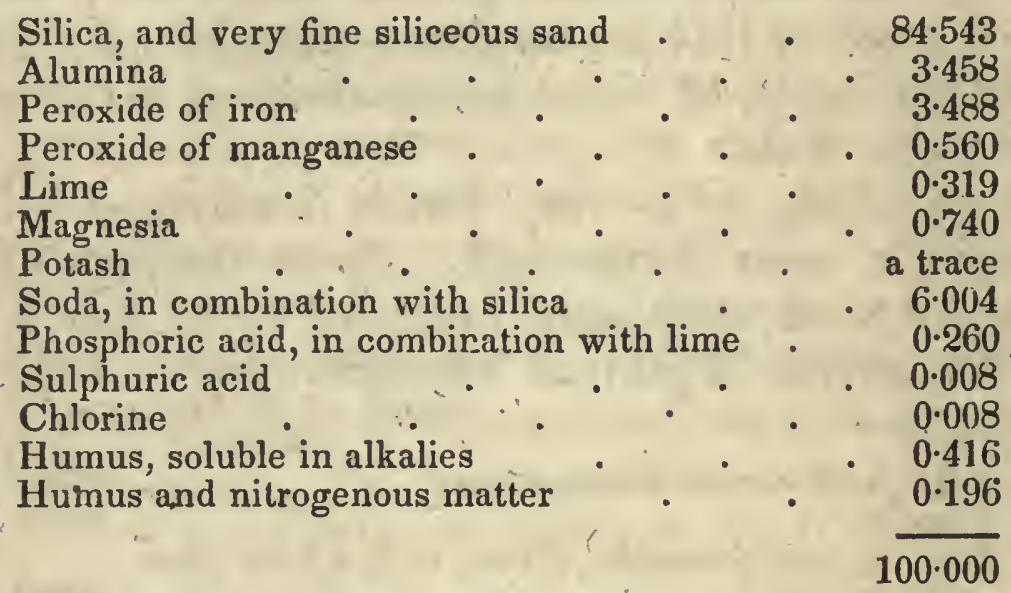

The portion of the soil subjected to analysis was taken at a depth of 10 inches, from a field which had received no manure for several years. It had previously produced in succession barley, beans, wheat, and grass, the latter for two years. The soil is remarkable, in a chemical point of view, from the large quantity of soda which it contains. Although the sulphuric acid, chlorine, and potash, are present in small quantity, yet this does not present any barrier to the development of the plants, as the surfacesoil is 18 inches in depth.

27. Analysis of a heavy alluvial soil in the vicinity of Norden. 100 parts contain;-

Silica, and very fine siliceous sand. 


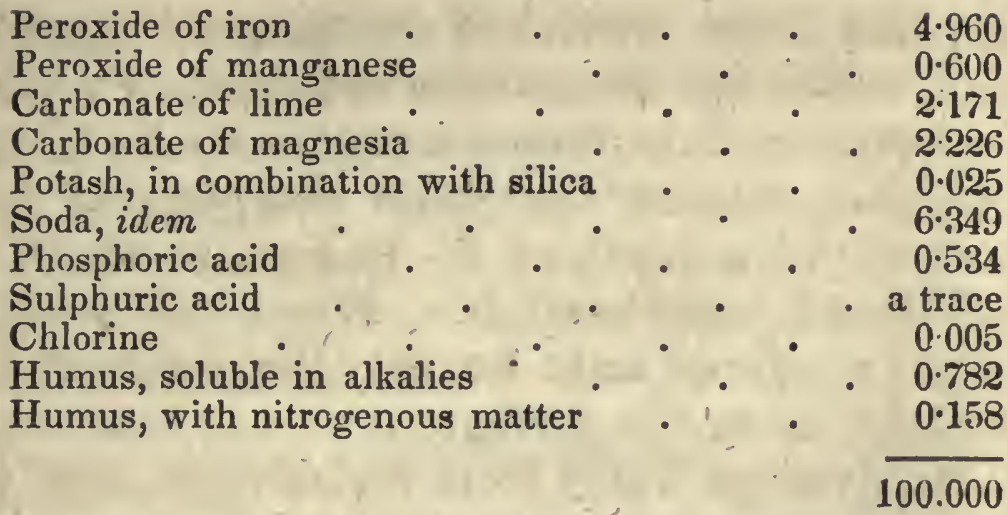

The specimen for analysis was taken at a depth of 10 inches from the surface of a field, which had been manured five years previously, and had produced since that time rape, rye, wheat, and beans. The crops of all these were plentiful, and of excellent quality. It is singular that this soil, which contains such a small proportion of gypsum, should be adapted for the cultivation of beans, and must be ascribed to the depth of the surface-soil. Yet, notwithstanding this, gypsum would form a beneficial manure to the land.

28. Analysis of very fertile alluvial soil, from Honigpolder; no manure had ever been applied to it. 100 parts contain :-

Siliceous sand separated by the sieve

Earthy portion of the soil

- $95 \cdot 5$

$100 \cdot 0$

100 parts of the latter consisted of :-

Silica, and fine siliceous sand . . $64 \cdot 800$

Alumina $\quad$ * $\quad$. $\quad$. $\quad 5.700$

Peroxide of iron . . . . . . .

Peroxide of manganese . . . . . 0.090

Lime . . . . . . 5.880

Magnesia $\quad . \quad 0 \quad 0 \quad 0.840$

Potash, principally in combination with silica $\quad 0 \cdot 210$

Soda, idem . . . . . 0.393

Phosphoric acid combined with lime . $\quad 0.430$

Sulphuric acid, idem _ . . . . 0.210

Chlorine (in common salt) _. . . . $\quad 0.201$

Carbonic acid, combined with lime _ . $\quad 3.920$

Humus soluble in alkalies . . . . 2540

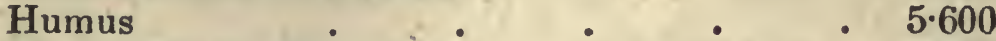

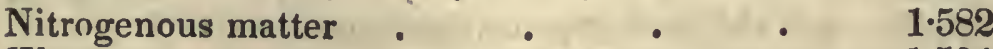

Water . . . . 1504 
Corn has been cultivated for seventy years upon this soil, which has never received dung or any other kind of manure; it is, however, occasionally fallowed. The subsoil retains the same composition as the surface-soil for a depth of 6-12 feet, so that it may be considered inexhaustible. When one portion of the soil is rendered unfit for use, the inferior layers are brought up to the surface.

29. Analysis of a soil from Rahdingen, near Balje. In this case the sea has assisted in the formation of the soil. The field yielded beautiful corn after being manured with stable dung, being particularly remarked for its fine crops of wheat, beans, and winter barley. 100 parts contain :-

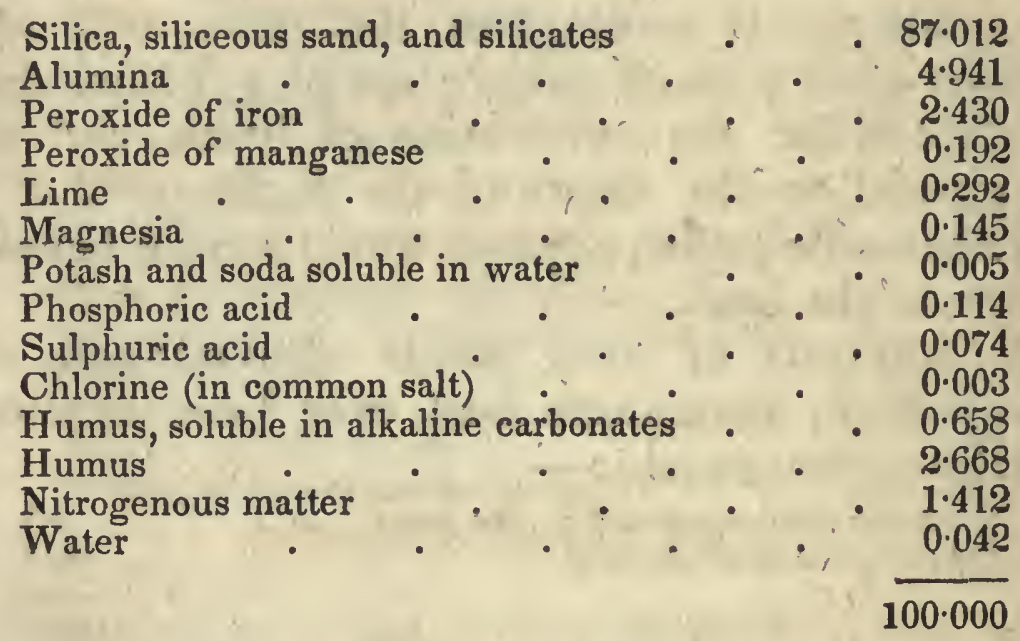

30. Soil of a field remarkable for producing large crops of hemp and horse-radish. 100 parts consisted of :-

Silica and siliceous sand

Alumina

Peroxide of iron

Peroxide of manganese

Lime

Magnesia

Potash

Soda

Phosphoric acid

Sulphuric acid

Chlorine

Humus, soluble in alkaline carbonates

Humus and nitrogenous matter

- $84 \cdot 021$

$4 \cdot 498$

$5 \cdot 120$

2.080

0.942

$1 \cdot 740$

0.050

0.012

0.482

0.012

$0 \cdot 008$

0.897

0.138 
31. Surface-soil of a field near Drackenburg; it produces very bad red clover. 100 parts contain:-

Silica, with very fine siliceous sand _ . $\quad$. 92.014

Alumina $\quad . \quad$. . . 2.652

Peroxide of iron . . . . 3.192

Peroxide of manganese . . . . . 0.480

Lime $\quad \therefore \quad . \quad 0.243$

Magnesia . . . . . 0.700

Potash combined with silica $\quad . \quad 0.125$

Soda, idem . . . . $\quad 0.026$

Phosphoric acid, in combination with lime $\quad 0.078$

Sulphuric acid . . . a a trace

Chlorine . . . . a trace

Humus and nitrogenous matter $\quad . \quad 0.150$

Humus, soluble in alkaline carbonates . . , 0.340 .

$100 \cdot 000$

The cause that clover will not flourish on this soil is probably due to the deficiency of gypsum and common salt.

32. Surface-soil of a field near Paddingbuttel. This field is particularly adapted for the growth of red clover. 100 parts consist of:-

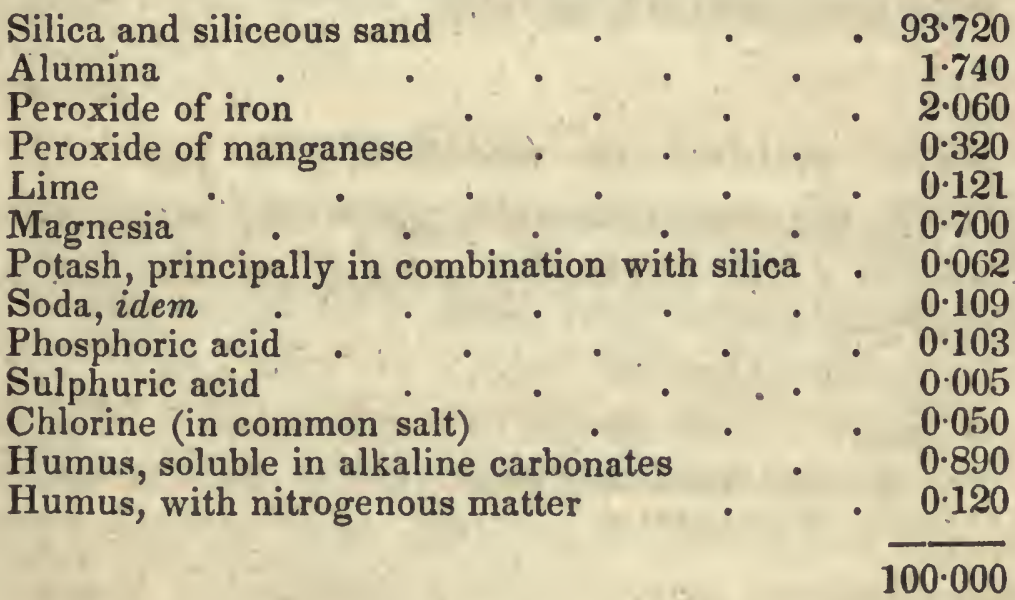

SOILS IN BOHEMIA.

33. Surface-soil of a very fertile field in the province of Dobrawitz and Lautschin. 100 parts gave

Silicenus sand, with much magnetic iron sand . $4 \cdot 286$

Earthy part separated by the sieve . $\quad 95 \cdot 714$ 
An aqueous infusion of the soil contained gypsum, common salt, magnesia, and humus. 100 parts of the soil gave:-

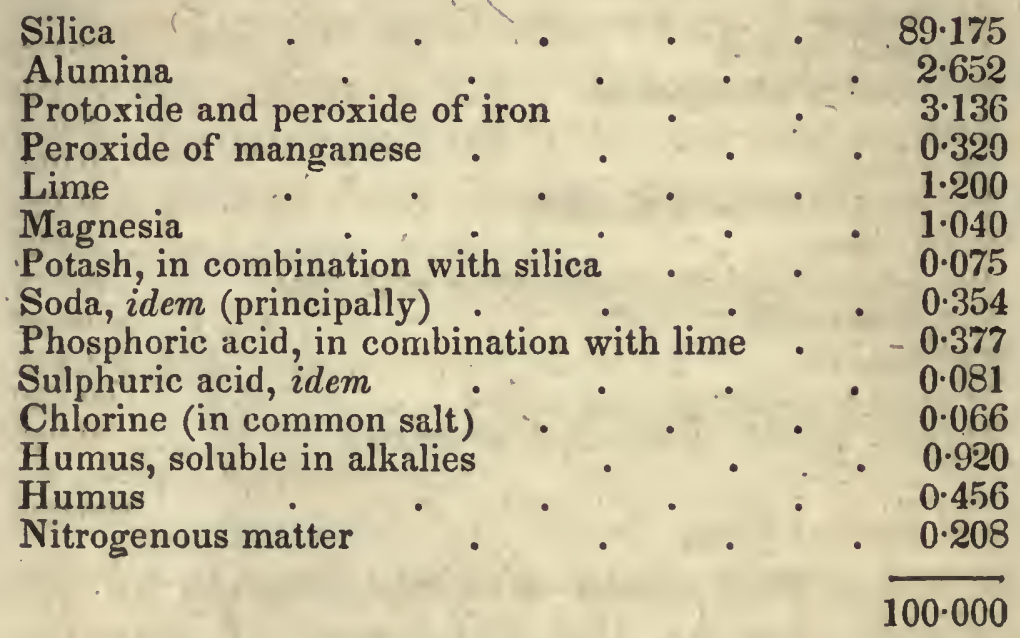

34. Surface-soil of a very fertile field in the province of Dobrawitz and Lautschin. 100 parts of the earth consisted of :-

Siliceous sand, with a little magnetic iron sand . $43 \cdot 780$

Finer part separated by the sieve

$56 \cdot 220$

$100 \cdot 000$

100 parts yielded to water 0.175 part of salts, consisting of common salt, gypsum, magnesia, and humic acid. 100 parts, by weight, of the earth consisted of :-

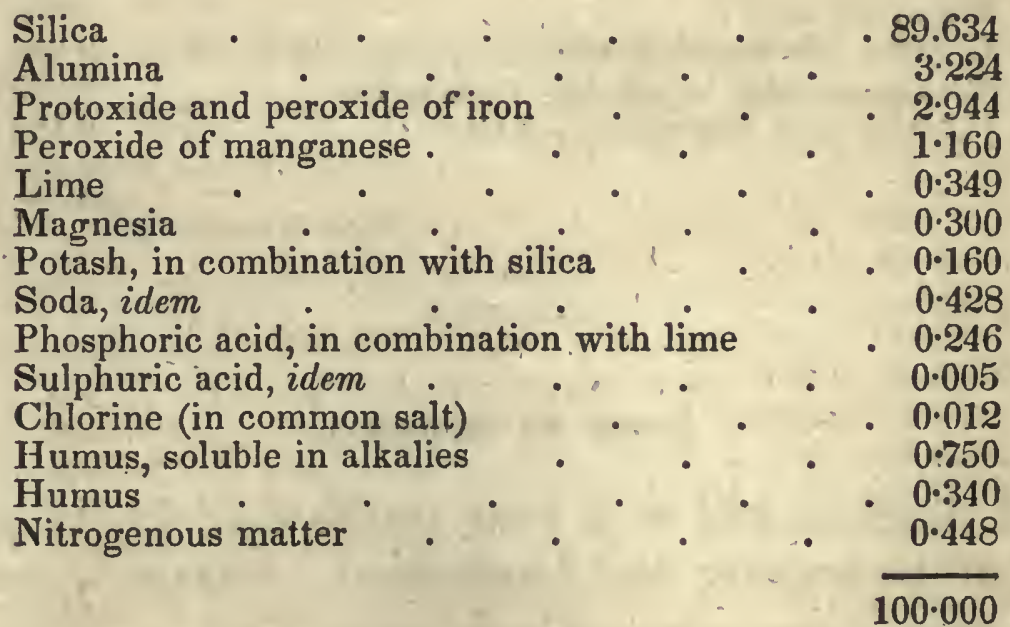

35. Analysis of a soil formed by the disintegration of basalt. 100 parts of the earth consisted of:- 
Siliceous sand, with very much magnetic iron sand 8.428

Earthy portion of the soil . . . . 91.572

$100 \cdot 000$

The aqueous infusion of the earth contained only traces of common salt and gypsum, with humus, lime, and magnesia. 100 parts consisted of:-

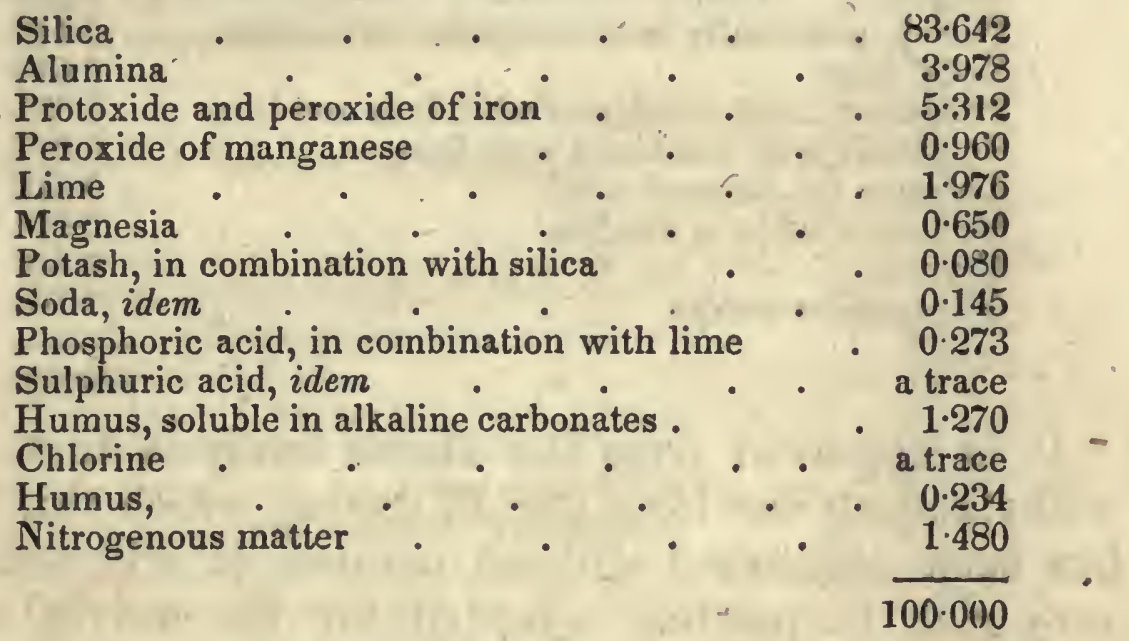

Manure consisting of gypsum, common salt, or ashes of wood, would be highly conducive to the fertility of this land.

\section{SOILS IN THE "MARKGRAFSCHAFT MAHREN."}

36. Surface-soil of a field very remarkable for its fertility. The field is called Haargraben, and is situated near the village of Nebstein. It has never been manured or allowed to lie fallow and yet has produced for the last 160 years the most beautiful crops ; thus furnishing a remarkable example of unimpaired fertility. $100 \cdot 000$ parts of this soil consisted of :

Coarse and fine siliceous sand, with a little magnetic iron sand

Earthy matter. $64 \cdot 600$

$100 \cdot 000$

100 parts of the earth yielded to water 0.010 sulphuric acid, 0.010 chlorine, 0.007 soda, $0.012 \mathrm{mag}-$ nesia, 0.010 potash, with a little silica, humus, and 
nitrogenous matter, but no appreciable trace of nitrates. 100 parts of the soil contained :-

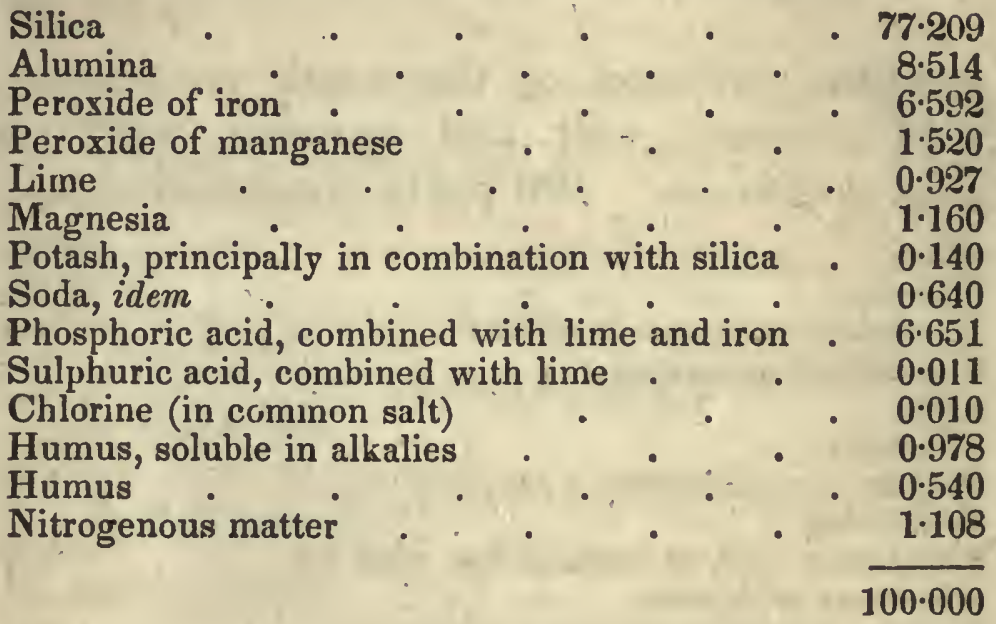

It is apparent from the above analysis that, notwithstanding the long period during which this land has been cultivated without manure, it still remains very rich in matters adapted for the nutrition of plants.

SOILS IN HUNGARY.

37. Analysis of a very fertile soil from Esákáng. 100 parts of the earth contained :-

Very fine siliceous sand
Earthy matter.

2.820

$97 \cdot 180$

$\overline{100 \cdot 000}$

The aqueous decoction of the soil contained principally gypsum, common salt, silica, magnesia, and humus. 100 parts of the soil yielded :-

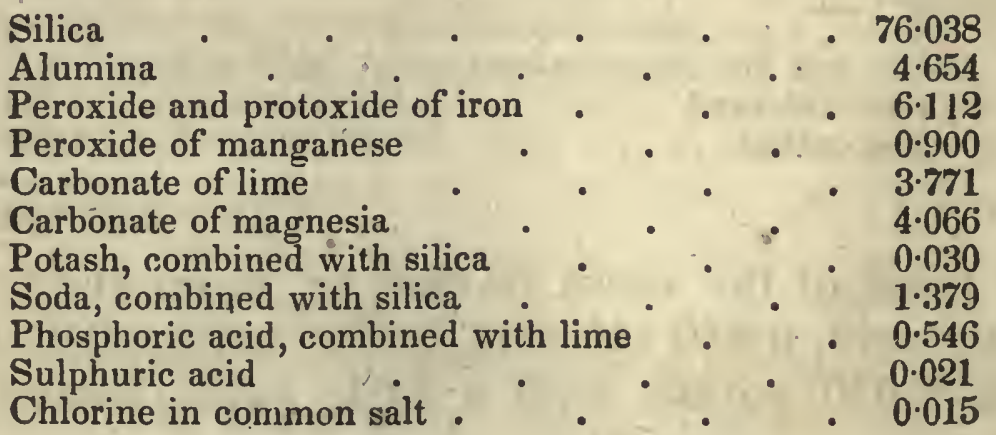


Humus, soluble in alkalies

Humus

Nitrogenous organic matter

$100 \cdot 000$

Subsoil of the same field at a depth of two feet. 100 parts consist of : -

Very fine siliceous sand with scales of mica

Earth separated by the sieve

-

100 parts of the earth contain :-

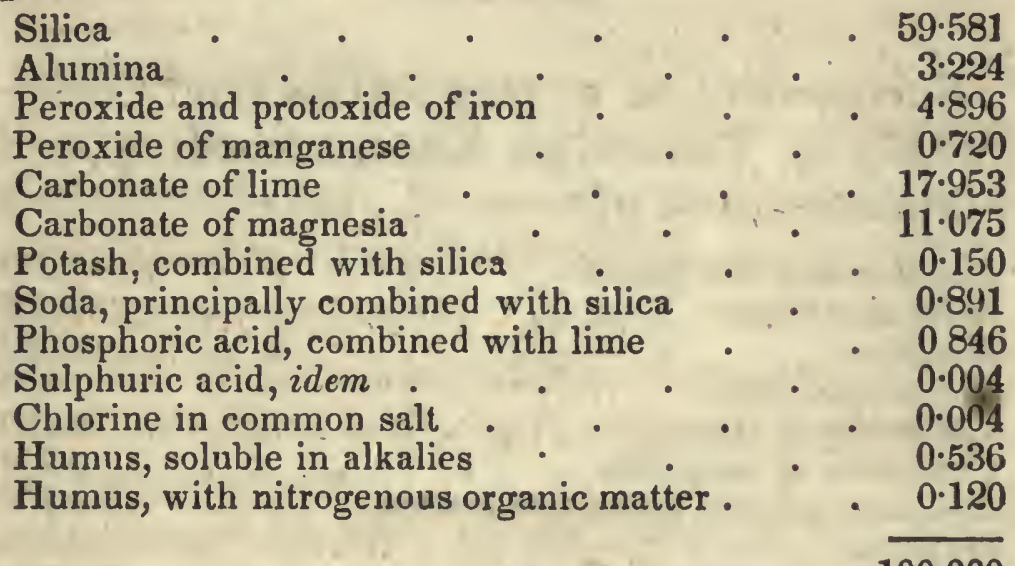

$100 \cdot 000$.

BELGIUM.

38. Surface-soil of a field distinguished for its fer-. tility. It had received no manure for twelve years previous to the time at which the analysis was executed. The rotation of crops for the latter nine years was as follows : -1 . beans, 2 . barley, 3. potatoes, 4. winter barley with red clover, 5 . clover, 6 . winter barley, 7. wheat, 8. oats; during the ninth year it was allowed to lie fallow. The soil is more clayey than loamy, and of a very fine grain. Water extracted from the soil, 0.013 soda, 0.002 lime, 0.012 magnesia, 0.009 sulphuric acid, 0.003 potash, 0.003 chlorine, with traces of silica and humus. 100 parts contained :-

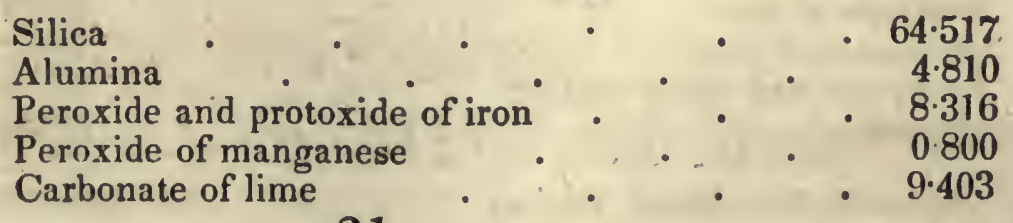


Carbonate of magnesia

Potash, principally combined with silica . $\quad$\begin{tabular}{rr}
10.361 \\
\hline
\end{tabular}

Soda

Phosphoric acid

Sulphuric acid

Chlorine

Humus

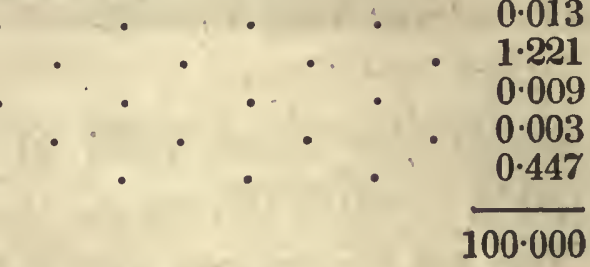

ENGLAND.

39. Surface-soil of a very fertile sandy field from the vicinity of Tunbridge, Kent, according to Davy. 100 parts consisted of:

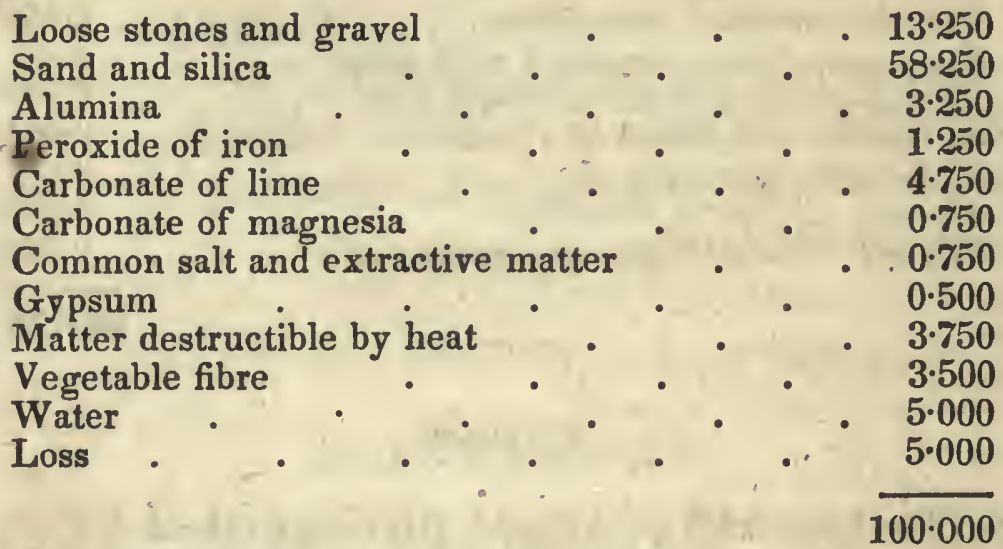

The great Davy, who was convinced of the importance of the inorganic constituents of soils, has omitted to detect the phosphoric acid, potash, soda, and manganese. All these must have been present in the soil, for we are informed that it produced good hops, for which these ingredients are indispensable.

40. A good turnip soil from Holkham, Norfolk, yielded to Davy:-

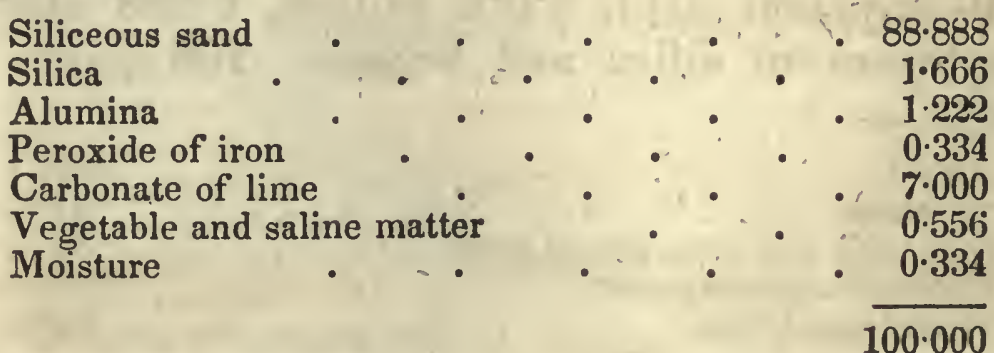


In this case also, phosphoric acid, manganese, potash, magnesia, \&c., have escaped detection by this acute chemist; yet doubtless they must be present in the soil, for we are informed that it produces good turnips.

41. An excellent wheat soil from the neighborhood of West Drayton, Middlesex, according to Davy. 100 parts contained :-

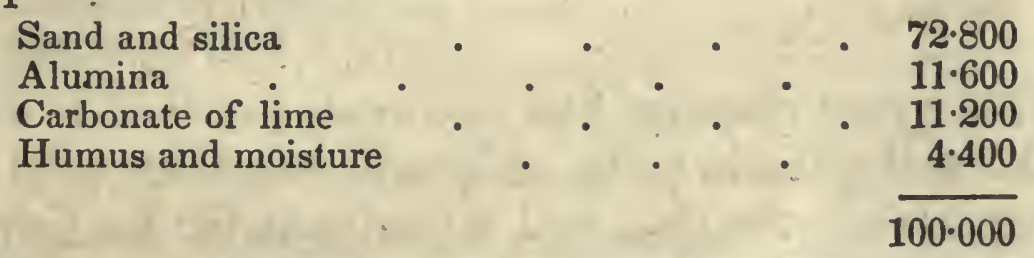

This analysis has been executed so imperfectly, that it only conveys a very feeble representation of the nature of the soil. A soil which bears good wheat must contain phosphate of potash, soda, chlorine, and sulphuric acid; yet none of these are exhibited by the analysis.

42. Surface-soil of a fertile field in the neighborhood of Bristol. 100 parts contained :-

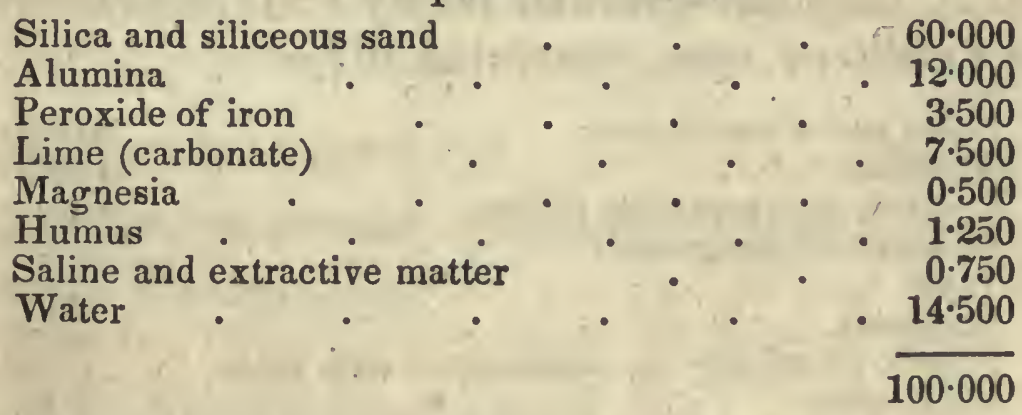

Davy has made several analyses of various fertile soils, and since his time numerous other analyses have been published; but they are all so superficial, and in most cases so inaccurate, that we possess no means of ascertaining the composition or nature of English arable land.

\section{SWEDEN.}

43. Surface-soil of a field which produces the most abundant crops, and has never been manured. (Berzelius.) 100 parts consist of:- 


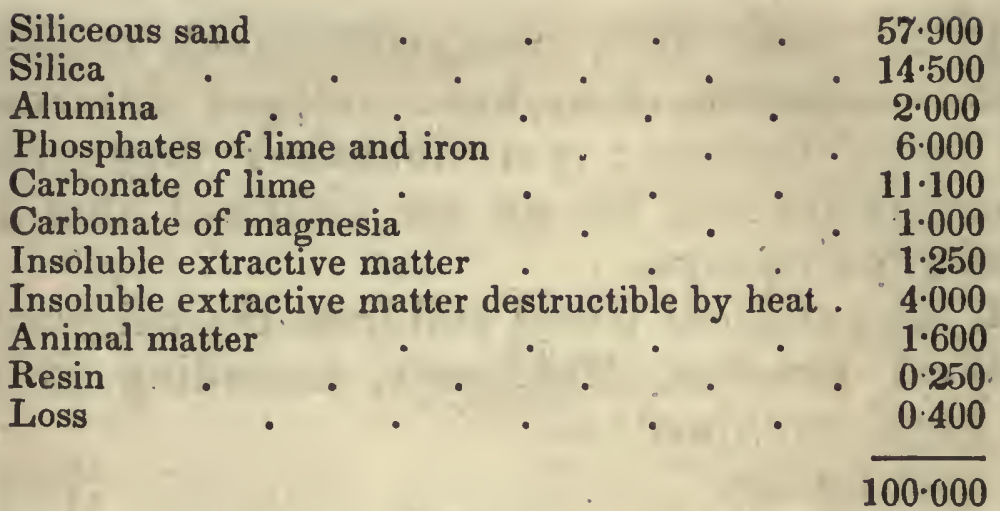

This great chemist has strangely omitted to detect in the soil potash, soda, chlorine, sulphuric acid, and manganese. As this soil is eminent for its fertility, there cannot be the slightest doubt that all these ingredients must have existed in it in notable quantity.

\section{ISLAND OF JAVA.}

44. A very fine-grained loamy soil, colored yellow by peroxide of iron, consisted of :-

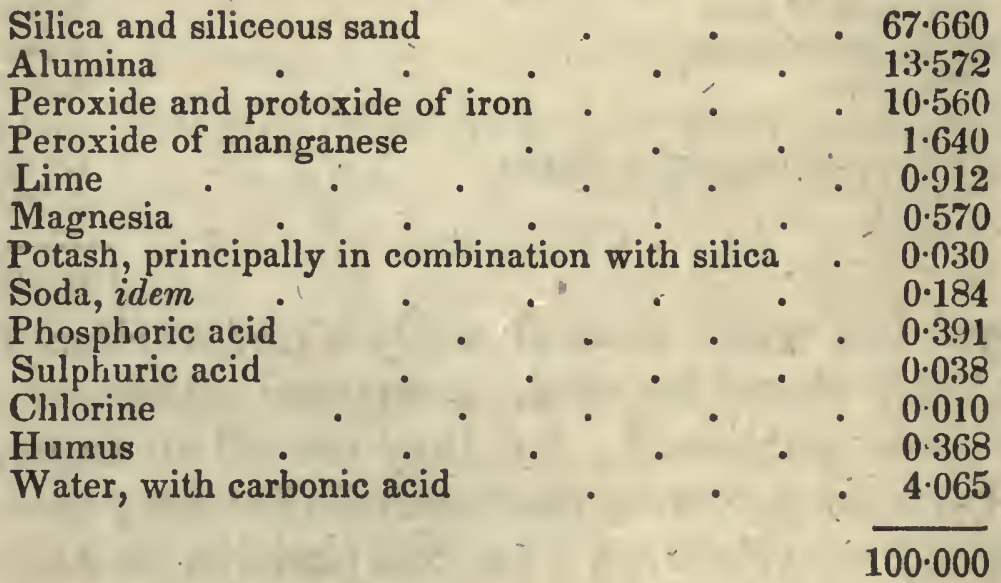

WEST INDIES (PORTO RICO).

45. Surface-soil of a very barren field. 100 parts contained :- 
Silica and siliceous sand $\quad . \quad$. 70.900

Alumina

6.996

Peroxide, and protoxide of iron (much magnetic iron sand)

Peroxide of manganese

$6 \cdot 102$

Lime

Magnesia

Potash

Carbonate of soda

Phosphoric acid, combined with lime

0.200

$2 \cdot 218$

$3 \cdot 280$

$0 \cdot 130$

$6 \cdot 556$

Sulphuric acid, combined with lime _ . 0.149

Chlorine in common salt . . . 0.067

Humus, soluble in alkalies _ . . 0540

Humus

$1 \cdot 500$

$100 \cdot 000$

This soil is improved by gypsum. Its sterility is due to the excessive quantity of carbonate of soda which is present.

\section{NORTH AMERICA.}

46. Surface-soil of alluvial land in Ohio, remarkable for its great fertility. 100 parts consisted of :-

Silica and fine siliceous sand . . . 79.538

Alumina

$7 \cdot 306$

Peroxide and protoxide of iron (much magnetic iron sand)

Peroxide of manganese

Lime

$5 \cdot 824$

$1 \cdot 320$

Magnesia

0.619

. 1.024

Potash, principally combined with silica $\quad 0.200$

Soda . . . . 0.024

Phosphoric acid, combined with lime and oxide of iron

Sulphuric acid, combined with lime .

$1 \cdot 776$

Chlorine

$0 \cdot 122$

Humus, soluble in alkalies

0.036

Nitrogenous organic matter

1.950

Wax and resinous matter

0.236

0.025

$100 \cdot 000$

47. (A.) Surface-soil of a mountainous district in the neighborhood of Ohio. (B.) analysis of the subsoil. This soil is also distinguished for its great fertility. 100 parts contain :- 


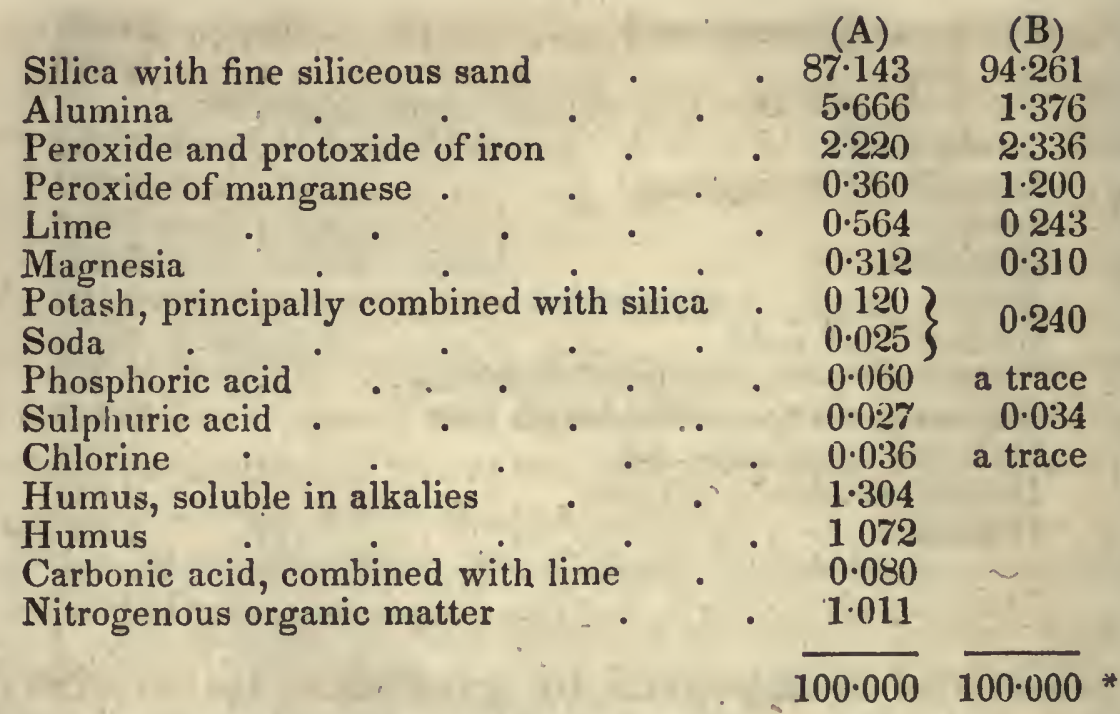

In the preceding part of the chapter we have inserted a number of analyses of various soils, as well as the conclusions deduced from them, by means of which the farmer may be enabled to ascertain the manures best adapted for each variety of soil. By inspecting the analyses of the sterile soils, it will be apparent that it is in the power of chemistry to point out the causes of their sterility. The general cause which conduces to the sterility of soils is either the absence of certain constituents indispensable for the growth of plants, or the presence of others, which

* Soil from Chelmsford, Massachusetts, on the Merrimack river, which has produced a large crop of wheat for 20 years, with only one failure, analyzed by Dr. Dana. 100 parts contain :-
Soluble geine
3.9228
$2 \cdot 6142$
Insoluble "
Sulphate of lime . $\quad . \quad+\quad . \quad 7060$
Phosphate of " • . . . . 9082
Silicates (silica, alumina, iron \&c.) • • . 91-8485

No trace of carbonate of lime, or of alkaline salts, could be discovered.

Soil from Maine, analyzed by Dr. Jackson, has produced 48 bushels of wheat per acre.

Water

Vegetable matter

Silica

Alumina
Subphosphate of alumina
Peroxide of

Peroxide of iron

Oxide of manganese .

Carbonate of lime 
exert an injurious or poisonous action. The analyses are those of Dr. Sprengel, - a chemist who has unceasingly occupied himself for the last twenty years in endeavoring to point out the importance of the inorganic ingredients of a soil for the development of plants cultivated upon it. He considers as essential all the inorganic bodies found in the ashes of plants. Now, although we cannot coincide with him in the opinion, that iron and manganese are indispensable for vegetable life, (for these bodies are found as excrementitious matter only in the bark, and never form a constituent of an organ,) yet we gratefully acknowledge the valuable services which he has rendered to agriculture, by furnishing a natural explanation of the action of ashes, marl, \&c., in the improvement of a soil. Sprengel has shown, that these mineral manures afford to a soil alkalies, phosphates, and sulphates; and further, that they can exert a notable influence only on those soils in which they are absent or deficient. In a former chapter of this book I have endeavored to point out the importance of considering these constituents as intimately connected with the vital processes of the vegetable organism, and have shown that the different families of plants contain unequal quantities of inorganic ingredients. This subject has been left . unexamined by Sprengel, yet it is one of much importance; for the application of manures must be regulated by the composition of the plants which are cultivated on any particular soil. Still, the composition of the soil must always be kept in view.' Thus it would be perfect extravagance to manure certain soils with marl, ashes, or gypsum; whilst, on the contrary, these compounds would produce the most beneficial results on other lands.

In a former part of the work, the principal action of gypsum upon vegetation was ascribed to the decomposition and fixation of the carbonate of ammonia contained in rain-water; but gypsum exerts a twofold action. The power of decomposing carbonate 
of ammonia, and of fixing the ammonia, is not peculiar to gypsum, but is shared also by other salts of lime (chloride of calcium, for example). But it acts also as a sulphate, and when useful as such cannot be replaced by any other salt of lime which does not contain sulphuric acid.

Hence gypsum can be replaced as a manure only by a mixture of a salt of lime with ammonia, and a salt of sulphuric acid. Sulphate of ammonia can therefore be substituted for gypsum, and exerts a more rapid and effectual action. In France, sulphuric acid has been poured upon the fields after the removal of the crops, and has been found to form a good manure. But this is merely a process for forming gypsum in situ; for the soils upon which it is applied contain much lime, which enters into combination with the sulphuric acid. It would certainly be much more advantageous to form sulphate of ammonia by adding the acid to putrefied urine, and to apply this mixture to the field. 


\section{APPENDIX TO PART I.}

EXPERIMENTS AND OBSERVATIONS ON THE ACTION OF CHARCOAL

FROM WOOD ON VEGETATION.

\section{BY EDWARD LUCAS.*}

"Iv a division of a low hothouse in the botanical garden at Munich, a bed was set apart for young tropical plants, but instead of being filled with tan, as is usually the case, it was filled with the powder of charcoal, (a material which could be easily procured,) the large pieces of charcoal having been previously separated by means of a sieve. The heat was conducted by means of a tube of white iron into a hollow space in this bed, and distributed a gentle warmth, such as tan communicates, when in a state of fermentation. The plants placed in this bed of charcoal quickly vegetated, and acquired a healthy appearance. Now, as always is the case in such beds, the roots of many of the plants penetrated through the holes in the bottom of the pots, and then spread themselves out; but these plants evidently surpassed in vigor and general luxuriance plants grown in the common way, - for example, in tan. Several of them, of which I shall only specify the beautiful Thunbergia alala, and the genus Peireskia, throve quite astonishingly; the blossoms of the former were so rich, that all who saw it affirmed, they had never before seen such a specimen. It produced also a number of seeds without any artificial aid, while in most cases it is necessary to apply the pollen by the hand. The Peireskice grew so vigorously, that the $P$. aculeata produced shoots several ells in length, and the $\boldsymbol{P}$. grandifolia acquired leaves of a foot in length. These facts, as well as the quick germination of the seeds which had been scattered spontaneously, and the abundant appearance of young Filices, naturally attracted my attention, and I was gradually led to a series of experiments, the results of which may not be uninteresting; 
for, besides being of practical use in the cultivation of most plants, they demonstrate also several facts of importance to physiology. The first experiment which naturally suggested itself, was to mix a certain proportion of charcoal with the earth in which different plants grew, and to increase its quantity according as the advantage of the method was perceived. An addition of $\frac{2}{3}$ charcoal, for example, to vegetable mould, appeared to answer excellently for the Gesneria and Gloximia, and also for the tropical Aroidece with tuberous roots. The first two soon excited the attention of connoisseurs, by the great beauty of all their parts and their general appearance. They surpassed very quickly those cultivated in the common way, both in the thickness of their stems and dark color of their leaves; their blossoms were beautiful, and their vegetation lasted much longer than usual, so much so, that in the middle of November, when other plants of the same kinds were dead, these were quite fresh and partly in bloom. Aroidece took root very rapidly, and their leaves surpassed much in size the leaves of those not so treated; the species which are reared as ornamental plants on account of the beautiful coloring of their leaves, (I mean, such as the Caladium bicolor, Pictum, Pacile, \&c.,) were particularly remarked for the liveliness of their tints; and it happened here also, that the period of their vegetation was unusually long. A cactus planted in a mixture of equal parts of charcoal and earth throve progressively, and attained double its former size in the space of a few weeks. The use of the charcoal was very advantageous with several of the Bromeliacece and Liliacece, with the Citrus and Begonia also, and even with the Palmoe. The same advantage was found in the case of almost all those plants for which sand is used, in order to keep the earth porous, when charcoal was mixed with the soil instead of sand; the vegetation was always rendered stronger and more vigorous.

"At the same time that these experiments were performed with mixtures of charcoal with different soils, the charcoal was also used free from any addition, and in this case the best results were obtained. Cuts of plants from different genera took root in it well and quickly; I mention here only the Euphorbia fastuosa and fulgens which took root in ten days, $\boldsymbol{P}$ andanus utilis in three months, $\boldsymbol{P}$. amaryllifolius, Chaincedorea elatior in four weeks, Piper nigrum, Begonia, Ficus, Cecropia, Chiococca, Buddleya, Hakea, Phyllanthus, Capparis, Laurus, Stiffica, Jacquinia Mimosa, Cactus, in 
from eight to ten days, and several others, amounting to forty species, including $1 / e x$ and many others. Leaves, and pieces of leaves, and even pedunculi, or petioles, took root and in part budded in pure charcoal. Amongst others we may mention the foliola of several of the Cycadea, as having taken root, as also did parts of the leaves of the Begonia Telfairia, and Jacararda brasiliensis ; leaves of the Euphorbia fastuosa, Oxalis Barrilieri, Ficus, Cyclamen, Polyanthes, Mesembryanthemum; also the delicate leaves of the Lophospermum and Martynia, pieces of a leaf of the Agave americana; tufts of Pinus, \&c. ; and all without the aid of a previously formed bud.*

"Pure charcoal acts excellently as a means of curing unhealthy plants. A Dorianthes excelsa, for example, which had been drooping for three years, was rendered completely healthy in a very short time by this means. An orange tree which had the very common disease in which the leaves become yellow, acquired within four weeks its healthy green color, when the upper surface of the earth was removed from the pot in which it was contained, and a ring of charcoal of an inch in thickness strewed in its place around the periphery of the pot. The same was the case with the Gardenia.

"I should be led too far were I to state all the results of the experiments which I have made with charcoal. The object of this paper is merely to show the general effect exercised by this substance on vegetation; but the reader who takes particular interest in the subject will find more extensive observations in the 'Allgemeine Deutsche Gartenzeilung' of Otto and Dietrich, in Berlin; or Loudon's Gardener's Magazine, for March, 1841.

"The charcoal employed in these experiments was the dust-like powder of charcoal from firs and pines, such as is used in the forges of blacksmiths, and may be easily procured in any quantity. It was found to have most effect when allowed to lie during the winter exposed to the action of the air. In order to ascertain the effects of different kinds of charcoal, experiments were also made upon that obtained from the hard woods and peat, and also upon

* The cuttings of several of these plants being full of moisture, require to be partially dried before they are placed in the soil, and are with difficulty made to strike root in the usual method. The charcoal is probably useful from its absorbing and antiseptic power. The Hakea is extremely difficult to propagate from cuttings. All the Laurus tribe are obstinate, some of them have not rooted under three years from the time of planting. - $W$. 
animal charcoal, although I foresaw the probability that none of them would ansiwer so well as that of pine wood, both on account of its porosity and the ease with which it is decomposed.*

"It is superfluous to remark, that in treating plants in the manner here described, they must be plentifully supplied with water, since the air having such free access penetrates and dries the roots, so that unless this precaution is taken the failure of all such experiments is unavoidable.

"The action of charcoal consists primarily in its preserving the parts of the plants with which it is in contact, - whether they be roots, branches, leaves, or pieces of leaves, - unchanged in their vital power for a long space of time, so that the plant obtains time to develop the organs which are necessary-for its further support and propagation. There can scarcely be a doubt also that the charcoal undergoes decomposition; for after being used five to six years it becomes a coaly earth; and if this is the case, it must yield carbon, or carbonic oxide, abundantly to the plants growing in it, and thus afford the principal substance necessary for the nutrition of vegetables. $\dagger$ In what other manner, indeed, can we explain the deep green color and great luxuriance of the leaves and every part of the plants, which can be obtained in no other kind of soil, according to the opinion of men well qualified to judge ? It exercises likewise a favorable influence by decomposing and absorbing the matters excreted by the roots, so as to keep the soil free from the putrefying substances which are often the cause of the death of the spongiola. Its porosity, as well as the power which it possesses of absorbing water with rapidity, and, after its saturation, of allowing all other water to sink through it, are causes also of its favorable effects. These experiments show what a close affinity the component parts of charcoal have to all plants, for every experiment was crowned with success, although plants

* M. Lucas has recently repeated these experiments, and found that the animal charcoal obtained by the calcination of bones possesses a decided advantage over all other kinds of charcoal, which he subjected to experiment. - Liebig's Annalen, Band xxxix. Heft I. S. 127.

t As some misconception has arisen regarding this explanation of the action of charcoal upon vegetation, and an.idea propagated, that the introduction of these opinions into this work incorporated them with those of Liebig, it is necessary to state that they are merely inserted here as part of the papers of M. Lucas. The true explanation has been given in a former part of the work, viz. that charcoal possesses the power of absorbing carbonic acid and ammonia from the atmosphere, which serve for the nourishment of plants. - ED. 
belonging to a great many different families were subjected to trial." (Buchner's Repertorium, ii. Reihe, xix. Bd. S. 38.)

\section{ON A MODE OF MANURING VINES.}

THE observations contained in the following pages should be extensively known, because they furnish a remarkable proof of the principles which have been stated in the preceding part of the work, both as to the manner in which manure acts, and on the origin of the carbon and nitrogen of plants.

They prove that a vineyard may be retained in fertility without the application of animal matters, when the leaves and branches pruned from the vines are cut into small pieces and used as manure. According to the first of the following statements, both of which merit complete confidence, the perfect fruitfulness of a vineyard has been maintained in this manner for eight years, and according to the second statement for ten years.

Now, during this long period, no carbon was conveyed to the soil, for that contained in the pruned branches was the produce of the plant itself, so that the vines were placed exactly in the same condition as trees in a forest which received no manure. Under ordinary circumstances a manure containing potash must be used, otherwise the fertility of the soil will decrease. This is done in all winecountries, so that alkalies to a very considerable amount must be extracted from the soil.

When, however, the method of manuring now to be described is adopted, the quantity of alkalies exported in the wine does not exceed that which the progressive disintegration of the soil every year renders capable of being absorbed by the plants. On the Rhine 1 litre of wine is calculated as the yearly produce of a square metre of land (10.8 square feet English). Now if we suppose that the wine is three-fourths saturated with cream of tartar, a proportion much above the truth, then we remove from every square metre of land with the wine only 1.8 gramme of potash. 1000 grammes ( 1 litre) of champagne yield only 1.54 , and the same quantity of Wachenheimer 1.72 of a. residue which after being heated to redness is found to consist of carbonates.

One vine-stock, on an average, grows on every square: 
metre of land, and 1000 parts of the pruned branches contain 56 to 60 parts of carbonate, or 38 to 40 parts of pure potash. Hence it is evident that 45 grammes, or 1 ounce, of these branches contain as much potash as 1000 grammes (1 litre) of wine. But from ten to twenty times this quantity of branches are yearly taken from the above extent of surface.

In the vicinity of Johannisberg, Rudesheim, and Budesheim, new vines are not planted after the rooting out of the old stocks, until the land has lain for five or six years in barley and esparsette or lucern; in the sixth year the young stocks are planted, but not manured till the ninth.

\section{ON THE MANURING OF THE SOIL IN VINEYARDS.*}

"IN reference to an article in your paper, No. 7, 1838, and No. 29, 1839, I cannot omit the opportunity of again calling the public attention to the fact, that nothing more is necessary for the manure of a vineyard than the branches which are cut from the vines themselves.

"My vineyard has been manured in this way for eight years, without receiving any other kind of manure, and yet more beautiful and richly laden vines could scarcely be pointed out. I formerly followed the method usually practised in this district, and was obliged in consequence to purchase manure to a large amount. This is now entirely saved, and my land is in excellent condition.

"When I see the fatiguing labor used in the manuring of vineyards, - horses and men toiling up the mountains with unnecessary materia!s, - I feel inclined to say to all, Come to my vineyard and see how a bountiful Creator has provided that vines shall manure themselves, like the trees in a forest, and even better than they! The foliage falls from trees in a forest, only when they are withered, and they lie for years before they decay; but the branches are pruned from the vine in the end of July or beginning of August. whilst still fresh and moist. If they are then cut into small pieces and mixed with the earth, they undergo

* Slightly abridged from an article by M. Krebs of Seeheim, in the "Zeitschrift für die landwirthschaftlichen Vereine des Grosherzogthums Hessen." No. 28, July 9, 1840. 
putrefaction so completely, that, as I have learned by experience, at the end of four weeks not the smallest trace of them can be found."

"Remarks of the - Editor. - We find the following notices of the same fact in Henderson's 'Geschichte dev' Weine der alten und neuen Zeit' :-

"'The best manure for vines is the branches pruned from the vines themselves, cut into small pieces, and immediately mixed with the soil.'

"These branches were used as manure long since in the Bergstrasse. M. Frauenfelder says : *

"I remember that twenty years ago, a man called Peter Müller had a vineyard here, which he manured with the branches pruned from the vines, and continued this practice for thirty years. His way of applying them was to hoe them into the soil after having cut them into small pieces.

" "His vineyard was always in a thriving condition; so much so, indeed, that the peasants here speak of it to this day, wondering that old Müller had so good a vineyard, and yet used no manure.'

"Lastly, Wilhelm Ruf of Schriesheim writes :

" For the last ten years I have been unable to place dung on my vineyard, because I am poor and can buy none. But I was very unwilling to allow my vines to decay, as they are my only source of support in my old age; and I often walked very anxiously amongst them, without knowing what I should do. At last my necessities became greater, which made me more attentive, so that I remarked that the grass was longer on some spots where the branches of the vine fell than on those on which there were none. So I thought upon the matter, and then said to myself: If these branches can make the grass large, strong, and green, they must also be able to make my plants grow better, and become strong and green. I dug therefore my vineyard as deep as if I would put dung into it, and cut the branches into pieces, placing them in the holes and covering them with earth. In a year I had the very great satisfaction to see my barren vineyard become quite beautiful. This plan I continued every year, and now my vines grow

* Badisches landwirthschaftliches Wochenblatt, v. 1834, S. 52 and 79. 
splendidly, and remain the whole summer green, even in the greatest heat.

" All my neighbors wonder very much how my vineyard is so rich, and that I obtain so many grapes from it, and yet they all know that I have put no dung upon it for ten years." "*

\section{ROOT SECRETIONS.}

IT should be stated, that the accuracy of the experiments of Macaire-Princep adduced by the author, page 164, is not generally admitted. Other chemists have been unable to obtain similar results, or if they do are inclined to ascribe them to injury of the roots of the plants examined. Professor Lindley in his notice of Liebig's work has remarked, that he has no fixed opinion on the subject, it being a question of facts and not of induction. Admitting root secretions, he nevertheless does not deem it necessary to look to the roots for these excretions, when we have so many proofs of their constant occurrence in other parts of a plant, as in the oily, resinous, waxy, acid, and acrid matter, from various parts of their surface, and in the peculiar substances lodged in the hollows of their stems or elsewhere, such as Tabasheer, in the bamboo. These are thought to be instances, "sufficient to satisfy the necessity of excretions occurring, and to render it superfluous to look to the roots for further aid in this particular."

The subject of excretion is one of great interest, and deserving of further examination. Several botanists have recently stated what are deemed fatal objections to the correctness of De Candolle's conclusions from Macaire's experiments. It is maintained, that the process of excretion from the roots of plants is not analogous to that of excretion in animals; that the deposits consist of materials which were in superabundance in the system of the plant, and that the reason why the same species of plants do not grow one after the other, is, that the first exhausted the soil of the materials necessary for the nourishment of the next. In some parts of the world, wheat crops are said to have been obtained fifty years in succession, where the supply of nutriment was sufficient. The application of the recent discovery of the means of coloring the wood of trees by

* The experiment has been made here with success $-W$. 
introducing coloring matters into their trunks, is reported to have shown that the coloring matters are thrown off from the roots, and plants growing near them have been poisoned, although the plant colored continued to grow. Report of British Association Meeting, August, 1841.

A series of experiments on this subject has been going on during five years in the Botanic Garden, Oxford, under the direction of Professor Daubeny. His object is to ascertain, " in the first place, how many successive years the soil may admit of the growth of the same crop, and, if it becomes deteriorated, at what rate the decrease of produce may proceed ; and, in the second place, what kind of vegetables will afterwards thrive best in soil, which, with reference to this particular crop, has become damaged, or effete.

"With a view to determine this, I have set apart, in one portion of our Botanical Garden, a number of distinct plots of ground, of known size, and uniform as to quality.

"These were in the first instance enriched with an equal amount of manure, and brought, as nearly as could be done, in every respect into a similar condition.

"Fifteen of these beds are planted year after year, without intermission, with the following crops: viz. potatoes, turnips, barley, oats, poppies (Papaver somniferum), buckwheat, tobacco (Nicotiana rustica), flax, hemp, endive, clover (Trifolium pratense), mint. (Mentha viridis), beans, parsley, and beet.

"The remaining fifteen beds receive in turn the same crops, but each year a different one is introduced; so that by comparing the amount of produce obtained each year from the first and second class of beds, - those in which the crop is permanent, and those in which it is made to shift about, - we may be enabled to learn, how much of any actual diminution ought to be attributed to the season, and how much to a deterioration or exhaustion of the soil.

"As it is scarcely five years since the experiments were commenced, the progress made has not yet been sufficient to render the results worth quoting; but should life and leisure be allowed me for bringing them to a conclusion, I trust some inferences may hereafter be deduced of utility to future husbandmen; although I should be far more sanguine with respect to the benefit that would accrue, if a piece of ground of greater extent were set apart for such experiments, as, under the auspices of any of our great Agricultural Societies, it might not be difficult to effect. 
"Should Science, indeed, succeed in settling the true cause of the deterioration of crops, and the most advantageous order of their succession, it is unnecessary for me to point out how important a boon she would confer upon the agriculturist.

"So extremely various, indeed, are the systems upon which the rotation is carried on in different countries, that no fixed principle would appear to regulate them, and the whole may be considered, as being founded much more upon the authority of long usage and tradition, than upon any actual comparison of the relative advantages of those resorted to in various places.

"This inquiry may therefore be pointed out, as being one of those lines of investigation, in prosecuting which the scientific chemist may be expected to benefit the practical farmer."

\section{PEAT COMPOST.}

(See p. 118, and 185.)

According to the statement of Messrs. Phinney and Haggerston, as contained in the Report on the Geological and Agricultural Survey of Rhode 1sland, by Dr. C. 'T. Jackson, a compost made of three parts of peat and one of stable manure, is equal in value to its bulk of clean stable dung, and is more permanent in its effects.

Dr. Jackson deems it essential that animal matters of some kind should be mixed with the peat, to aid the decomposition and produce the requisite gases. Lime decomposes the peat, neutralizes the acids, and disengages the ammonia. The peat absorbs the ammonia, and becomes in part soluble in water. The soluble matter, according to $\mathrm{Dr}$. Jackson, is the apocrenate of ammonia; crenate of ammonia and crenate of lime being also dissolved. With an excess of animal matter and lime, free carbonate of ammonia is formed.

The peat should be laid down in layers with barn-yard manure, night-soil, dead fish, or any other animal matter, and then each layer strewed with lime. In Dr. Jackson's Report, he has presented highly valuable results from the use of this compost, which deserve the attention of every agriculturist. He gives the following details of the man- 
ner in which the compost was prepared upon the farm of Mr. Sandford, near the village of Wickford in North Kingston. "In the corner of the field a cleared and level spot was rolled down smooth and hard, and the swamp muck was spread upon it, forming a bed eight feet wide, about fifteen or twenty feet long; and nine inches thick. For every wagon load of the muck one barrel of fish was added, and the fish were spread on the surface of the muck, and allowed to become putrescent. The moment they began to decompose, he again covered them with peat, and a renewed layer of fish was spread and covered in the same manner. The fermentation was allowed to proceed for two or three weeks, when the compost was found to have become fit for the land. To this he was advised to add lime in the proportion of one cask to each load of compost early in the spring, which it was supposed would complete the decomposition in two or three weeks. Such a heap should be rounded up and covered, so as to prevent the rain washing out the valuable salts, that form in it. And in case of the escape of much ammonia, more swamp muck or peat should be spread upon the heap, for the purpose of absorbing it." Dr. Jackson is of opinion, that the phosphoric acid of the peat and animal matter would convert the lime into a phosphate, and thus approximate it very closely to bone manure. - Report, p. 170.

Any refuse animal matter can be, of course, employed in a similar manner. "The carcass of a dead horse, which is often suffered to pollute the air by its noxious effluvia, has been happily employed in decomposing 20 tons of peat earth, and transforming it into the most enriching manure." - Young's Letters of Agricola, Letter 25, p. 238.*

Night soil may be composted with peat with great advantage, sufficient lime being added to deprive it of odor; large quantities of ammonia are given off and absorbed. $\dagger$

Appended to Dr. Jackson's Report will be found a letter

* In a Report on a Reẻxamination of the Geology of Massachusetts, 1838, Dr. Dana particularly notices the evolution of ammonia from fermenting dung, and supposes that the ammonia combines with geine to form a soluble compound. See Note to page 83 of the Report.

$f$ Night-Soil. The quantity of night-soil collected and removed from the city of Boston annually, is about four hundred thousand square feet. It is used by cultivators in the immediate vicinity; being composted with soil, lime, peat, \&c. Large quantities of animal matter from slaughter-houses, and other sources, are also made use of. The heaps are left exposed, uncovered to the air, and the value of the compost is consequently greatly diminished. See page 199. 
from E. Phinney, Esq., of Lexington, well known as one of the most skilful agriculturists, "On the reclaiming of peat bogs and the employment of peat as manure."

\section{SOURCE OF THE CARBON OF PLANTS. (FROM DAUBENY'S}

LECTURES ON AGRICULTURE, 1841.)

(See Chapter II.)

"UNTIL within the last century, it would have been taken for granted, that the soil was the source from whence proceeded all the solid matter at least which entered into the constitution of a plant, and there were several circumstances which tended to countenance such an opinion. No plants, it was observed, would continue long to thrive in earth unmixed with some proportion of vegetable mould, and the fertility of the latter is greatly enhanced by the addition of animal or vegetable matter, in that state of decay, in which it becomes soluble in water, and therefore fitted to obtain admission into the vessels of plants.

"Hence, when Priestley had demonstrated, that leaves decompose the carbonic acid of the atmosphere, giving out its oxygen and assimilating its carbon, the doctrine alluded to still to a certain extent maintained its ground; and it was even questioned by Ellis and others, whether in fact, if we were to strike the balance between the opposite influence of a plant during the day and the night, as much carbonic acid might not be exhaled by it at one period, as had been decomposed at another.

"I was therefore induced myself to undertake some experiments, ${ }^{*}$ the results of which appear to establish, that plants, even in a confined atmosphere, do in reality add a great deal more oxygen to the air than they abstract from it, whilst the amount of carbonic acid which may be introduced undergoes at the same time a corresponding diminution.

"This effect I even found to take place in diffused light, as well as under the direct influence of the solar rays, and to be no less common in aquatic than in terrestrial plants.

"I also showed, that when a branch loaded with flowers, as well as with leaves, was introduced into a jar.containing 
a certain proportion of carbonic acid, the balance still continued to be in favor of the purifying influence of the vegetáble.

"The apparatus I made use of consisted of a large bellglass jar, containing in one case 600 , in another 800 cubic inches of air,* and suspended by pulleys. Its edges dipped into quicksilver, contained in a double iron cylinder of corresponding dimensions to the jar, which, being closed at bottom, constituted a well of about six inches in depth, calculated to receive a fluid, and to admit of the glass vessel moving freely in it. The inner margin of this hollow cylinder was cemented air-tight, according as circumstances required, either to a plate of iron, or to a pot of the same material upon or in which the plant operated on might be placed; and the jar was then let down upon it, until its edges were sunk a little beneath the surface of the mercury.

"Thus all communication with the external atmosphere was cut off, and the effect of the plant upon the air inclosed in the jar wás readily measured, by simply pressing down the latter, and thus expelling a portion of its contents through a tube, communicating with its interior, and introduced at its outer extremity under a pneumatic trough, wherein the air might be collected and examined. By connecting this extremity with a vessel containing a measured quantity of carbonic acid, and raising the jar a little in the well of mercury, it was easy to draw in any proportion of that gas, with which it was thought proper that the plant should be supplied. A portion of the air was always tested, immediately after the introduction of every fresh portion of carbonic acid, and again after an interval of some hours, and the proportion of this gas and of oxygen present was each time carefully registered. The amount of carbonic acid was determined by a solution of potass, that of oxygen by the rapid combustion of phosphorus with a portion of it introduced into a bent tube.

"Such was the mode of procedure, when an entire plant became the subject of experiment; but some of the most satisfuctory trials were with branches of certain shrubs, themselves too large to be admitted under the jar. "These branches, without being detached from the parent trunk, were introduced through a hole in the centre of two corresponding semicircular plates of iron, which were cemented air-tight, to the inner margin of the iron cylinder on the

" * Larger jars', containing from 1200 to 1300 cubic inches were latterly employed." 
one hand, and to the stem of the branch on the other. In this manner, when the jar came to be placed over them, and to dip beneath the surface of the mercury, the external air was as effectually excluded, as when the whole of the plant had been enclosed.

"6s The results of several experiments conducted after this plan are given in a tabular form in the Memoir; but it may be sufficient here to specify one of the most satisfactory of those undertaken. In this case the jar itself contained about 600 cubic inches of air, and the plant experimented on was the common lilac (syringa vulgaris). The proportion of carbonic acid in the jar was each morning made equivalent to five or six per cent. by additions through the tube.

"The first day no great alteration in the air was detected, but on the second day, by eight in the evening, the oxygen had risen to 26.5 per cent. In the mornïng it had sunk to $26 \cdot 0$, but by two $\mathrm{P}$. M. it had again risen to no less than 29.75 , and by sunset it had reached 30.0 per cent. At night it sunk one half per cent.; but the effect during the following day was not estimated, as the sickly appearance which the plant now began to assume induced me to suspend the experiment.

"In a second trial, however, the branch of a healthy lilac growing in the garden was introduced into the same jar, where it was suffered to remain until its leaves becarne entirely withered.

"The first day the increase of oxygen in the jar was no more than 0.25 per cent., but on the second it rose to $25 \cdot 0$. At night it sunk to nearly 22.0 per cent., but the next evening it had again risen to $27 \cdot 0$. This was the maximum of its increase, for at night it sunk to 26.0 . and in the morning exhibited signs of incipient decay. Accordingly in the evening the oxygen amounted only to 26.5 ; the next evening to 25.5 ; the following one to 24.75 ; and the one next succeeding it had fallen to the point at which it stood at the commencement, or to $21^{\circ} 0$ per cent.

"The reason of this decrease was, however, very manifest from the decay and falling off of the leaves; so that this circumstance does not invalidate the conclusion which the preceding experiments concur in establishing, nanely, that in fine weather a plant, so long at least as it continues healthy, adds considerably to the oxygen of the air when carbonic acid is freely supplied.

"In the last instance quoted, the exposed surface of all 
the leaves enclosed in the jar, which were about fifty in number, was calculated at not more than 300 square inches, and yet there must have been added to the air of the jar as much as 26.0 cubic inches of oxygen, in consequence of the action of this surface upon the carbonic acid introduced.

"But there is reason to believe, that even under the circumstances above stated (which appear more favorable to the due performance of the functions of life than those to which Mr. Ellis's plants - were subjected), the amount of oxygen evolved was much smaller than it would have been in the open air, for I have succeeded, by introducing several plants into the same jar of air in pretty quick succession, in raising the amount of oxygen contained from twenty-one to thirty-nine per cent., and probably had not even then attained the limit to which the increase of this constituent might have been brought.

"How great then must be the effect of an entire tree in the open air under favorable circumstances! and we must recollect that, cceteris paribus, the circumstances will be favorable to the exertion of the vital energies of the plant, within certain limits at least, in proportion as animal respiration and animal putrefaction furnish to it a supply of carbonic acid.

"These experiments were published in the Philosophical Transactions for 1836, and have been noticed in Dr. Lindley's popular Introduction to Botany ; neither am I aware that the deductions which were drawn from them have anywhere been disputed."

Source of the Hydrogen of Plants; from Daubeny's Lectures.

(See Chapter IV.)

"It would seem, I think, from the late important researches of M. Payen, that the decomposition of water commences subsequently to that of carbonic acid, whether it be, that the former process requires a greater development and energy in the vegetable functions, or that it takes place in organs of a different description and of later growth.

"M. Payen seems to have established, that under the general term of ligneous fibre, or lignin, we have hitherto confounded at least two distinct substances, namely, that which constitutes the walls of the cells, and that which, by being deposited afterwards on the surfaces of the latter, 
imparts to them the solidity of texture which woody fibre possesses.

"He has succeeded in isolating the two by chemical means, and has found, that whilst the cellular matter has exactly the same composition as starch, being composed of 44.9 carbon, $6 \cdot 1$ hydrogen, 49 oxygen, or 44.9 carbon and 55.1 of water; the incrusting matter afterwards formed consists of 53.76 carbon, 40.2 oxygen, and 6 of hydrogen, or of 53.76 carbon, 45.2 of water, and 1 of hydrogen.*

"The composition of the ligneous matter of different kinds of wood will therefore vary according to the relative proportion of these two ingredients, as is shown in the following table of M. Payen:-

\begin{tabular}{|c|c|c|c|c|}
\hline LIGNEOUS Bodies. & Carbon. & Hydrogen. & Oxygen. & $\begin{array}{l}\text { Incrusting } \\
\text { matter. }\end{array}$ \\
\hline Incrusting matter of the wood & $53 \cdot 76$ & $6 \cdot 00$ & $40 \cdot 20$ & 100 \\
\hline Wood of Saint Lucia & $52 \cdot 90$ & 6.07 & $41 \cdot 03$ & 90 \\
\hline Ebony . & $52 \cdot 85$ & $6 \cdot 00$ & $41 \cdot 15$ & 89 \\
\hline Walnut . & $51 \cdot 92$ & $5 \cdot 96$ & $42 \cdot 12$ & 82 \\
\hline Oak . . . . & $50 \cdot 00$ & 6.20 & $43 \cdot 80$ & 61 \\
\hline $\begin{array}{l}\text { Ditto according to Gay-Lus- } \\
\text { sac, and Thénard }\end{array}$ & $51 \cdot 45$ & $5 \cdot 82$ & $42 \cdot 73$ & \\
\hline Beech . . & $49 \cdot 25$ & $6 \cdot 10$ & $44 \cdot 65$ & 52 \\
\hline Cellular matter . & $44 \cdot 90$ & $6 \cdot 10$ & 4900 & 00 \\
\hline
\end{tabular}

"This then proves, that, in the formation of the matter which incrusts and fortifies the walls of the cellular tissue in wood, though not in that of the cellular tissue itself, a decomposition of water must have taken place; since the 1 per cent. of hydrogen which Payen has found in excess, can only have arisen in this manner.

"This increase of hydrogen becomes still greater, when, in the progress of vegetation, the plant begins to secrete oils, camphors, and other analogous bodies, products, which, it is to be remarked, abound most within the tropics, where the light of the sun is most intense.

"Hence the decomposition of water, no less than that of carbonic acid, seems due to solar influence, and accordingly, the greater sweetness of subacid fruits, in a warm than in a cold summer, arises from the transformation of a larger amount of tartaric or other vegetable acids into sugar, owing to that separation of oxygen from the former which is accomplished by the agency of light.

"The process of assimilation of plants in its most simple

" * Payen has since stated, that this incrusting matter probably consists of two or three different principles." 
form may therefore be stated, as consisting in the extrication of hydrogen from water, and of carbon from carbonic acid, in consequence of which one of three things must happen, - either all the oxygen of the water and of the carbonic acid are separated, as in those bodies which, like caoutchouc, volatile oils, \&c., consist of nothing else but carbon and hydrogen; or, secondly, only a part of it is exhaled, as in the case of the incrusting matter of wood, and in sugar; or, thirdly, that belonging to the carbonic acid alone is decomposed, whilst the water remains, as in starch and cellular tissue."

Dependence of the nutritive Qualities of Plants on their Nitrogen; from Daubeny's Lectures.

(See page 139.)

"The dependence of the nutritive qualities of various articles of food upon the proportion of nitrogen is well shown in a recent memoir of Monsieur Boussingault, * who gives, on the authority of the celebrated agriculturist $V$ on Thaer, a scale of the relative degree of nutriment afforded by various plants to cattle, and then places by the side of it a statement of the proportion of azote present in them, from which it appears, that the nutritious quality of each bears a pretty constant ratio to the quantity of nitrogen they contain.

"This may be seen by the following table:

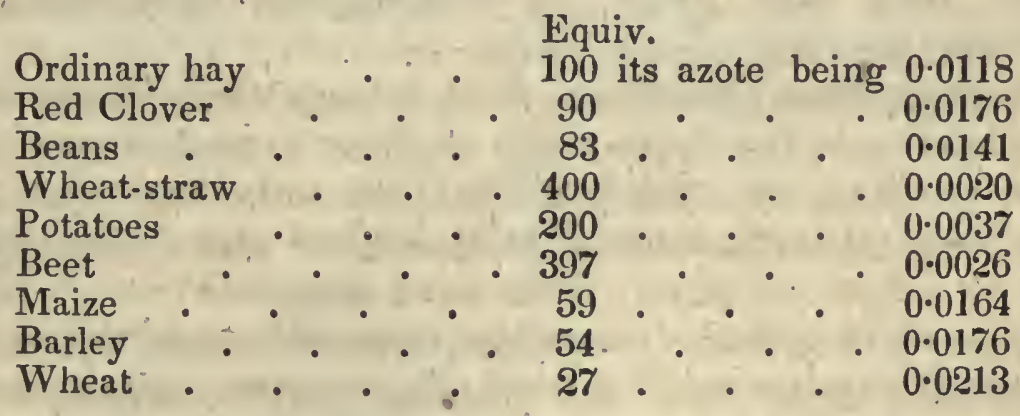

"When we reflect, indeed, that animal matter, which so abounds in nitrogen, is nevertheless derived, either directly or indirectly, from vegetable, it follows, as a necessary consequence, that existence can only be maintained by the aid of those principles in plants, which contain a certain proportion of the element alluded to. 
"And this has been shown by the experiments of Magendie upon dogs, which were fed_on sugar, starch, gum, and other substances destitute of nitrogen, and in a very short time pined away and died."

Difference between different Plants in their power of decomposing Ammonia; from Daubeny's Lectures.

\section{(See Chapter V.)}

"It may be inferred, from some experiments made by Boussingault, that a great difference exists between plants in their power of assimilating nitrogen, and to this difference that chemist is disposed to attribute the advantage of alternately growing what are called fallow crops, for the purpose of refreshing the soil.

"'During germination,' he remarks, 'the quantity of azote which seeds contain appears to be on the increase, but there is this curious difference between different kinds, that whilst those of leguminous plants, sown in pure earth and moistened with nothing but distilled water, obtained an increase of nitrogen which the atmosphere alone could have afforded, those of barley and other cerealia remained in that respect stationary, unless manure were afforded.'

"Boussingault also shows in a subsequent memoir, that peas, clover, and other legumes absorb azote, even when planted in a soil that contains no decomposing animal or vegetable matter, but that the cerealia, although if so placed, they may grow, do not appear to secrete this principle.

"Boussingault, however, does not go so far as to maintain, that the latter in no stage of their existence are capable of discharging this function, but only that the plant must have already arrived at a higher state of vigor, in order to derive its supply from such a source.

"It is on the same principle, that although the animal in general obtains its food from the various organic bodies. on which he subsists, yet that in an early stage of existence, before his organs are fitted for undergoing the labor of assimilating such materials, nature has provided him in his mother's milk with aliment already almost elaborated.

"It is thus, too, that in the seed the embryo is surrounded with a mass of albumen, from which it derives its support, until its roots become sufficiently vigorous to extract nourishment from the ground.

"Hence it becomes in most cases necessary, that crops 
cultivated as articles of food should have access to vegetable or animal manure from which they may derive their azote, but as this supply would soon be exhausted, were it not at the same time regenerated from the atmosphere, we see the advantage of intercalating a green fallow crop ploughed into the ground with others; as leguminous plants, according to the experiments of Boussingault, have the greatest power of absorbing nitrogen from the air.

"On the same principle this chemist suggests the introduction of the Jerusalem artichoke into light soils, which, owing to the entire absence of mould, appear irreclaimably barren; this vegetable, the tubers of which afford nourishment to cattle almost equal to potatoes, having great power of absorbing both carbon and nitrogen from the air, and thus by degrees generating a certain amount of soil.*

"I have seen this vegetable very commonly cultivated for the use of cattle, in the light lands of the Grand Duchy of Baden, and in certain parts of Alsace.

"But if it be true, as Liebig has endeavored to establish, that plants obtain every thing except their alkalies and earthy constituents from the atmosphere, what, it may be asked, becomes of the theory that attributes the unfitness of a soil for yielding several successive crops of the same plant to the excretions given out by its roots?

"For if plants receive the whole of their volatilizable ingredients from the atmosphere, these excrementitious matters, being composed chiefly of carbon, hydrogen, and oxygen, will not be absorbed, and therefore cannot affect the succeeding vegetation.

"The above inference would seem unavoidable, if it were considered absolutely proved, that nothing but the fixed ingredients of a plant were derived from the earth, but this is not fully established, even with respect to the humus, much less with respect to the more soluble matters which the soil contains.

"These latter, there seems no reason for doubting, may be taken up by the spongioles of the roots dissolved in

"** It is to be observed, that Boussingault attributes to plants the power of absorbing nitrogen from the air, but he alleges no proof that they have that power, and his results may be just as well explained by supposing them to have different powers of absorbing ammonia. It is to be remarked, that the helianthus tuberosus belongs to a tribe of plants remarkable for their power of absorbing and exhaling water, and hence it is evident, that they will be brought into contact within a given time with a larger amount of ammonia, than other plants, which possess a less degree of energy in that respect." 
water, together with the alkaline and earthy ingredients which are derived from the soil, nor am I aware of any proof that they may not likewise be assimilated when so introduced.

"The theory of M. Decandolle, therefore, is not affected by the above experiments, but must rest on its own merits, and continue to afford a subject for inquiry to the scientific agriculturist."

\section{Practical Inferences. From Dr. Daubeny's Lectures on Agriculture, delivered at Oxford, 1841.}

"The first inference that may be drawn, relates to the utility of diligent and frequent tillage, in order to favor the disintegration of the soil, and the free admission to it of oxygen and of water.

"Unless the former take place, no fresh alkali can be extracted from the subjacent rock by the action of water upon it; unless the latter be brought about in a sufficient degree, the humus excluded from air cannot undergo that process of eremacausis, or gradual combustion, on which its influence upon the nutrition of plants has already been shown to depend.

"Hence, in ancient times, the importance attached to those operations which had this object for their aim, -

"'Quid est agrum bene colere?' asked Cato. 'Bene arare. Quid secundum? Arare. Quid tertium? Stercorare.'

Thus ploughing was regarded the most important process in agriculture, after which, though at a long interval, came manuring.

"The design, therefore, of the agriculturist is, to reduce the soil to that loose and crumbling condition, in which it becomes entirely pervious, to air and moisture, imparting to it the quality which the ancients denominated putre.

" ' Et cui putre solum, (namque hoc imitamur arando,) Optima frumentis.'

"Hence the superiority of spade husbandry over the plough, if the expense of the labor be not taken into the account; hence the fertility of the small farms of the ancient Romans, notwithstanding their rude methods and their deficiency of skill ; hence the fine condition of those tracts of land, which are subjected to the unremitting manual exertions of societies of men'like the Trappists, whose mis- 
taken views of religion have led them into that entire isolation from human society, under which even the severest physical toil becomes itself a relief.

"The same principle explains in some degree the utility of subsoil-ploughing, which, by bringing up to the surface a portion of earth previously out of the reach of those influences which tend to cause its disintegration, extracts from it the alkaline and other ingredients required by the plant for its subsistence.

"It is found advantageous, in the first instance, merely to break and pulverize the subsoil to a depth of eighteen or twenty inches, without bringing it to the surface, and only after a lapse of four or five years to mix it with the vegetable mould above, a practice, the utility of which depends, not only on the mechanical condition of the land being rendered more favorable to culture in consequence of its becoming more friable, but likewise, probably, owing to the chemical decomposition of its component parts having taken place more completely.

"Other circumstances, such as its influence on the drainage of the land, will no doubt coöperate in producing the benefit which often results from the practice of subsoiling; but that the cause pointed out really contributes to its efficacy, may be inferred from a fact attested by many experienced agriculturists, * namely, that those soils are most benefited by subsoil-ploughing, which can be rendered thereby more pervious to moisture, and consequently to air ; whilst those which contain too large a percentage of clay to be affected in this manner by the process, derive no advantage from it.

"But it must not be forgotten, that the utmost pains bestowed upon its elaboration cannot generate any new principles, but only act, by enabling the soil to impart more readily to the crop those which it already possesses.

"This obvious truth will explain the cause of the disappointment felt by farmers, at finding, that after a certain time, the most diligent tillage no longer affords them the same returns as it did at first.

"It it said, that Jethro Tull, who first proved the advantages of deepening and pulverizing soils, was nevertheless obliged at length to admit, that at each repetition of the experiment the success was less decided, unless manure were at the same time applied. Judicious tillage, in short, 
like the use of machinery in the arts, does not create any new power, but only tends to render more available those already latent in the earth.

"It was not therefore without reason, that Cato, after, as we have seen, pronouncing, that the first, and the second thing in agriculture, is to plough, adds, that the third is to manure, for what is this but the art of providing for the intended crop an adequate supply of those ingredients which enter into its composition?

"The principles therefore which have been laid down, whilst they will serve to guide the husbandman in the selection of his fertilizers, may also explain the different results that are obtained from the use of the same kind of mineral manure in different soils.

"Among those which have excited the greatest interest within the last few years, may be mentioned the nitrates of potass, and of soda.

"The former, commonly called saltpetre, is produced spontaneously in most parts of the world, and especially in hot countries, in consequence of animal and vegetable decomposition conducted under particular conditions, and accordingly it has been introduced into agriculture from an early period.*

"The latter, sometimes distinguished from its crystalline form, as cubic nitre, is met with in large quantities in Peru, fourteen leagues from the port of Iquicque, where, according to Mr. Darwin, $\uparrow$ it forms a stratum two or three feet thick, lying close beneath the surface, and following the margin of a grand basin or plain, elevated 3300 feet above the level of the Pacific, but which, nevertheless, appears evidently to have been at one time a lake, or inland sea.

"The price of the salt at the ship's side in 1835, at the time Mr. Darwin visited the spot, was fourteen shillings a cwt., the grand item of expense being its transport to the coast. I

6* Where the price operates as an objection to its use, the method of forming artificial nitre-beds, by mixing together vegetable and animal matters in a state of decomposition with calcareous earth, may be economically adopted. See Cuthbert Johnson, on Saltpetre and Nitrate of Soda, Ridgway, 1840."

" + See Darwin's Journal, in Voyage of the Beagle:"

₹ Mr. J. H. Blake of Boston, who recently visited Peru, informs me, that the cost of the nitrate of soda was $\$ 2.50$ per quintal, and that it could be obtained here at from $4 \frac{1}{2}$ to 5 cents per lb. The crude nitrate, containing from 70 to 80 per cent. of the pure salt, might be obtained here at $2 \frac{1}{2}$ cents per $1 \mathrm{~b} .-W$. 
"These particulars are perhaps not unimportant, as they may serve to show that an almost unlimited supply of both these salts may be calculated upon, and, in the case of the nitrate of soda, that its price might be kept down, rather than enhanced, by an increased demand.

"That, however, with which the agriculturist is most concerned, is to determine the relative value of these salts as manures, and to discriminate the kind of land to which either or both are beneficial.

"Now, it is remarkable, that the nitrates, whilst they have in some cases occasioned a wonderful increase of produce, in others have appeared of little service, and also that, whereas on certain land both were equally efficacious, on a different description of soil, the one has answered, whilst the other failed.

"For a great deal of interesting information on this subject, I may refer to the Journal of the Royal Agricultural Society of England, - its last number* more especially : on the present occasion I shall confine myself to noticing the communication of Mr. Hyett, of Painswick, as one, which probably points to the true cause of the advantage derived from the employment of these salts.

"'Mr. Hyett's experiments were made upon the stone or cornbrash of Gloucestershire, a coarse and impure oolitic limestone, which had been drilled with white Sicilian wheat in the autumn.

"Nitrate of soda, at the rate of 1 cwt. to the acre, was on the 21st of April, sown and hoed in over all the field, excepting two square portions, which were staked out; and left unnitrated.

"On the 16th of May the effect of the salt was perceived, by the dark green color of the plants.

"'The results of the harvest were as follows :

\begin{tabular}{|c|c|c|c|}
\hline Produce. & $\begin{array}{c}\text { Measur } \\
\text { Without nitrate. }\end{array}$ & $\begin{array}{l}\text { acre. } \\
\text { With nitrate. }\end{array}$ & $\begin{array}{l}\text { Value per acre. } \\
\text { Excess. }\end{array}$ \\
\hline $\begin{array}{c}\text { Corn clean } . \\
\text { tail } \ldots\end{array}$ & $\begin{array}{ccc}\text { Bu. } & \text { Pks. } & \text { Pts. } \\
30 . & 2 . & 11 \\
2 . & 3 . & 11\end{array}$ & $\begin{array}{rrr}\text { Bu. } & \text { Pks. } & \text { Pts. } \\
37 . & 3 . & 4 \\
5 . & 3 . & 7\end{array}$ & $\begin{array}{ccc}\text { Bu. } & \text { Pks. } & \text { Pts. } \\
7 . & 0 . & 9 \\
2 . & 3 . & 12\end{array}$ \\
\hline - total & 33. 2.6 & 43. 2. 11 & $10 . \quad 0.5$ \\
\hline & Weight. & Per acre. & \\
\hline Straw & 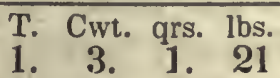 & $\begin{array}{llll}\text { T. } & \text { Cwt. } & \text { qrs. } & \text { lbs. } \\
\text { 1. } & \text { 11. 2. } & \text { 2. }\end{array}$ & 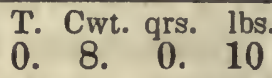 \\
\hline
\end{tabular}

"From these data Mr. Hyett calculates, that the increased value of the produce, arising from the use of the 
nitrate of soda, gives a profit of $2 l .17 s .2 d$. per acre, after deducting $1 \mathrm{l}$. 3s. $0 d$. for the value of the salt employed.

"But not only does the nitrate increase the quantity of the grain, but it tends to augment those ingredients, which contain the largest amount of nitrogen, and consequently afford the greatest degree of nutriment, namely, the gluten and albumen.

"This is shown, by the analysis of the nitrated, and nonnitrated wheat, made by a chemist at his request, the results of which were as follows :

\begin{tabular}{|c|c|c|}
\hline t & $\begin{array}{l}\text { Wheat on which the } \\
\text { nitrate was used, gave }\end{array}$ & $\begin{array}{l}\text { Wheat on which no } \\
\text { nitrate was usad, gave }\end{array}$ \\
\hline Bran . . . . . & $25 \cdot 000$ & $24 \cdot 000$ \\
\hline Gluten ...... & $23 \cdot 250$ & $19 \cdot 000$ \\
\hline Starch ...... & $49 \cdot 500$ & $55 \cdot 500$ \\
\hline Albumen .... & $1 \cdot 375$ & $.6: 25$ \\
\hline Extract ..... & .375 & .250 \\
\hline \multirow[t]{2}{*}{ Loss and water. . } & $\cdot 5$ & $\cdot 628$ \\
\hline & $100 \cdot$ parts. & 100. parts. \\
\hline
\end{tabular}

"Thus it is seen, that in the nitrated wheat there was 4.25 per cent. more gluten, and 0.75 more albumen, than in the non-nitrated sample.

"Considering, then, that these constituents contain nearly 16 per cent. of nitrogen, we are justified perhaps in attributing their increase to the decomposition of the nitric acid present in the salt, and the consequent supply of nitrogen in greater abundance than is naturally present in the soil.

"And if such be the mode of its operation, it may be possible to explain why these salts should appear so capricious in their effects on the different kinds of land to which they have been applied.

"When the ground already contains all the other constituents which the plant requires, as, for instance, a sufficient amount of the earthy phosphates, and of silicate of potass, the addition of the nitric salt will do good, by supplying nitrogen, and thus enabling the vegetable to assimilate a proportionate quantity of the other ingredients.

"But when the latter are already nearly exhausted, the addition of the nitrates will no slonger be of advantage, since only that portion of nitrogen can be assimilated which is equivalent to the amount of the earthy phosphates, of the silicate of potass, and of the other fixed ingredients, which the plant obtains from the soil. 
"Hence, the proper remedy in such a case would seem to be, that of applying some other manure, which may furnish a due supply of the deficient matters.

"Thus, if the nitrates have failed, we should be inclined to try the next year the effect of phosphate of lime, or of animal manure, upon the same soil.

"But it seems to happen sometimes, that the same land, which is benefited by the administration of one kind of nitric salt, is scarcely affected by another.

"This anomaly presented itself in an experiment on a small scale, which was tried at my request, by my brother, the Rev. E. Daubeny, on his farm, in the vicinity of Cirencester.

"The subsoil is a stiff retentive clay, resting upon the cornbrash limestone, and the farm, before it came into its present occupation, was in an exhausted condition, though it has latterly yielded somewhat better returns.

"A coarse analysis of a sample, conducted according to the method recommended by Mr. Rham, in the Journal of the English Agricultural Society, ${ }^{*}$ afforded me the following results :

"1000 grains contained, 607, of impalpable powder, consisting of

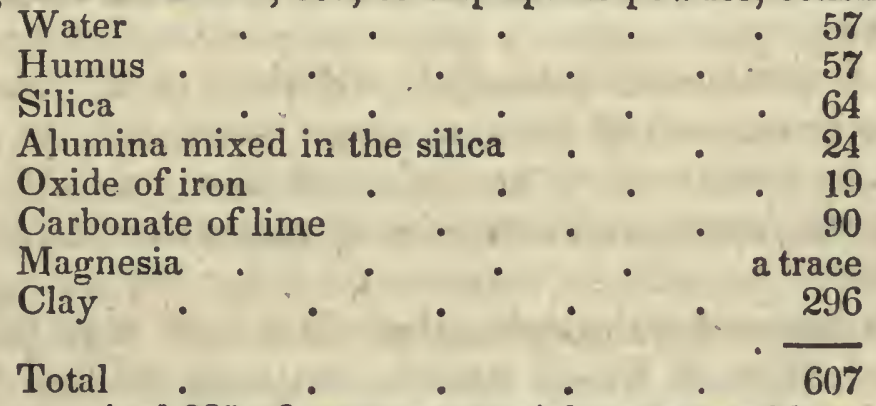

And 388 of coarser materials, separated by

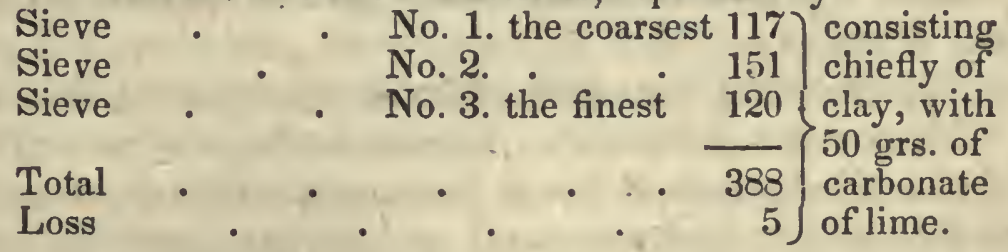

"Four equal strips of this land, each somewhat exceeding $\frac{1}{4}$ of an acre, and contiguous one to the other, which had been sown with wheat in the autumn of 1839 , were measured out.

"The first of these, which lay next to the hedge, was left without any addition of manure.

"The second, adjoining, had a top-dressing of $\frac{1}{4} \mathrm{cwt}$. of . nitrate of potass given it in April. 
"The third portion was left, like the first, without addition.

"The fourth, or that farthest from the hedge, had a similar top-dressing of nitrate of soda applied at the same period.

"The salts were respectively scattered over the strips of land in as uniform a manner as possible, and became diffused through the soil, by means of the showers which followed shortly after their application.

"As the wheat advanced towards maturity, the nitrated patches were distinguishable, by the more vivid greenness of the crop, and by its standing up somewhat above the general level, but this difference was less perceptible at a later stage of its progress.

"In the autumn the whole was reaped as usual, and the following results obtained :

"No. 1. produced only 5 bushels, $54 \mathrm{lbs}$. of grain, or 23 bushels, 36 lbs. to the acre, but the crop had been accidentally trodden by sheep, and much devoured by birds. The straw was not weighed.

"No. 2. produced 7 bushels, 57 lbs.; or 31 bushels, 48 lbs. to the acre, and $520 \mathrm{lbs}$. of straw $=1 \mathrm{ton}, 0 \mathrm{cwt}$. $80 \mathrm{lbs}$. to the acre.

"No. 3. produced 6 bushels, 54 lbs., or 27 bushels, 36 lbs. to the acre, and $421 \mathrm{lbs}$. of straw $=16 \mathrm{cwt}$. to the acre. "No. 4. produced 6 bushels, $48 \mathrm{lbs}$., or 27 bushels, $12 \mathrm{lbs}$. to the acre, and $432 \mathrm{lbs}$. of straw $=15 \mathrm{cwt}$. $48 \mathrm{lbs}$. to the acre.

"With respect to weight, that of No. 2. was $62 \frac{1}{2}$ lbs. to the bushel, that of No. 3. and 4. was only $62 \mathrm{lbs}$.

"Now $3 \frac{1}{2}$ lbs. of flour from No. 3, produced of bread 4 lbs. 4 ozs.

"Whereas $3 \frac{1}{2}$ lbs. from No. 2. produced 4 lbs. 14 ozs.

"Hence the difference, between the produce of the strip of ground which had been manured with nitrate of potass, and that which had received no manure, may be calculated as follows:

"Amount of produce, of

Bu. lbs.

"No. $3 \quad 6 \quad 54=6$.

"No. $2 \quad 7 \quad 57=7$.

"As $6: 7:: 100: 120$, or 20 per cent. of increase in the amount of produce.

"To which add, that the quantity of flour, that in No. 
3 had produced $4 \mathrm{lbs} .4 \mathrm{ozs}$. of bread, in No. 2. produced 4 lbs. 14 ozs. Now

"As 4 lbs. 4 ozs. : 4 lbs. 14 ozs. : : $100: 114$.

"Showing an increase per cent. of $14+20=34$ per cent.

"Now if we calculate the wheat as worth eight shillings a bushel, the profit of using the nitrate of potass will stand as follows :

" 27 bushels $36 \mathrm{lbs}$. at $8 \mathrm{~s} .=11 \mathrm{l}$. value of the produce on the non-nitrated land : add 34 per cent. or $\frac{1}{3} \mathrm{rd}=3 \mathrm{l}$. $13 \mathrm{~s}$. $4 d$., for the value of the nitrated, which, after deducting 1l. 10s. for the value of a cwt. of nitrate of potass, and for carriage, will leave to the farmer a clear profit of $2 l$. $3 s .4 d$.

"The superior absorbing power of the nitrated flour, over the non-nitrated, was found to depend upon the presence of a larger amount of gluten, for I discovered in the former $\mathbf{7 4 0}$ grs. in the pound, or 13 per cent.; in the latter $850 \mathrm{grs}$. in the pound, or 15 per cent. of that ingredient, the difference being 2 per cent. in favor of the nitrated wheat, a result which confirms, in a very satisfactory manner, the statement of Mr. Hyett.*

"But how are we to account for the failure of the nitrate of soda, on soil whish had been so materially benefited by the administration of nitrate of potass?

"The small scale upon which the experiment was conducted, may render us reluctant to build much upon the results obtained, until it has been again repeated, but supposing the fact to be hereafter confirmed, I can only conjecture, that the difference must have arisen from a deficiency in the land, of potass, which would be supplied by the saltpetre, but not by the nitrate of soda. $\uparrow$ Should this be the true solution, those soils, in which nitrate of soda has succeeded, ought to contain a larger quantity of potass, than those in which it has failed.

“The general principles laid down may also inform us, as to the true plan upon which the succession of our crops should be regulated.

"Those plants ought to succeed each other, which contain different chemical ingredients, so, that the quantities

"* The amount of gluten is smaller than in the samples reported on by Mr. Hyett, but my gluten was dried, with the greatest care, under the exhausted receiver of an air-pump, with sulphuric acid, till it ceased to lose weight."

" $\uparrow$ Nitrate of soda is stated to exist in barley, but it has not been detected in wheat. It would therefore be worth while to see, whether the above salt is particularly suited to the former crop." 
of each, which the soil at any given time contains, may be absorbed in an equal ratio.

"Thus a productive crop of corn could not be obtained, without the phosphates of lime and magnesia which are present in the grain, nor without the silicate of potass which gives stability to the stalks.

"It would be injudicious, therefore, to sow any plant that required much of any of the above ingredients, immediately after having diminished the amount of them present in the soil, by a crop of wheat, or of any other kind of corn.

"But, on the other hand, leguminous plants, such as beans, are well calculated to succeed to crops of corn, because they contain no free alkalies, and less than one per cent. of the phosphates.

"They thrive, therefore, even where these ingredients have been withdrawn, and during their growth, afford time for the ground to obtain a fresh supply of them, by a further disintegration of the subjacent rock.

"For the same reason, wheat and tobacco may sometimes be reared in succession in a soil rich in potass, because the latter plant requires none of those phosphoric salts which are present in wheat.

"In order, however, to proceed upon certain data, it would be requisite, that an analysis of the plants most useful to man should be accomplished in the different stages of their growth, a labor which has hitherto been only partially undertaken, and which perhaps is an object worthy to engage the attention of a great Body, like that of the English Agricultural Association.

"It is a curious fact, that the same plant differs in constitution when grown in different climates. Thus in the beet-root, nitre takes the place of sugar, when this plant is cultivated in the warmer parts of France.*

"The explanation of this difference is probably as follows:

"Beet-root contains, as an essential ingredient, not only saccharine matter, but also nitrogen, and it is probable, that the two are mutually so connected together in the vegetable tissue, that the one cannot exist without the other. The nitrogen, being derived from the decomposition of ammonia, must be affected by any cause which diminishes the supply of the latter; and in proportion as this ingredient is wanting, the secretion of sugar will likewise fall off.

"Now, it has been shown by Liebig, that the formation 
of nitric acid is owing to the decomposition of ammonia, and it is conceived by him, that the last products of the decomposition of animal bodies present themselves, in the form of ammonia in cold, and in that of nitric acid in warm climates. * Hence, in proportion to the amount of nitric acid formed, and of nitre absorbed by the plant, that of the nitrogen, and consequently that of the saccharine matter, present in it, may be diminished.

"We may also be guided in the management and selection of manures, by the principles above laid down. The solid excrement of animals varies of course in composition according to the nature of their food: thus that of herbivorous animals, which are fed principally on grasses, contains much silicate of potass, as well as phosphoric salts, but comparatively little nitrogen; whilst human. fæces contain little of the former ingredient, but much phosphate, and a larger proportion of nitrogen. There will be seen even a difference in these respects between the manure afforded by the inhabitants of towns, fed principally upon animal food, and that of peasants, who subsist in a greater degree upon vegetables.

"In like manner, the excrement of cattle is more efficacious as manure, when the animal is well fed, and undergoing the fatting process, than when it is more scantily nourished.

"According to Sprengel, there is a difference between different kinds of herbivorous animals in this respect, cows

* "I have seen no attempt to account for the formation of nitrate of soda in such large quantities in Peru, and may therefore offer the following, as at least a plausible solution.

"Wherever salt lakes occur, which become partially or wholly dried up during a part of the year, carbonate of soda will be formed from the decomposition of common salt. This I have observed myself on the sandy plains of Hungary, in the neighborhood of Pesth. Now if any circumstances should concur in such spots, calculated to generate nitric acid, the latter, by its stronger affinity for the alkali, would take the place of the carbonic acid, and nitrate of soda would result.

"This, however, being a deliquescent salt, would not accumulate on the surface, except in countries like Peru, remarkable for their extreme dryness.

"But how are we to account for the generation of so large a quantity of nitric acid in this locality?

"If we suppose with Mr. Darwin, that the district in which the salt is found was once a lake or inland sea, its change to dry land must have caused the destruction of all its marine inhabitants. Now the decomposition of their exuviæ would, in a warm climate, present themselves, as stated in the text, in the form, rather of nitric acid, than of ammonia.

"Hence the production of so much nitrate of soda in Peru, is attrib. utable to the heat; its preservation to the dryness of the climate." 
requiring, for the chemical constitution of their body, or for the formation of their milk, more nitrogen, and more phosphate of lime, than sheep; whilst the latter require again more sulphur, and more common salt, for the formation of their wool. Hence the excrements of oxen contain less nitrogen than those of sheep, whilst they are more abundant in salt and sulphur.

"Accordingly it is found in practice, that sheep's dung ferments more readily than that of black cattle. "The latter, therefare,' says Liebig, 'is of most service on soils consisting of lime and sand, which contain no silicate of potass or phosphates, whilst their value is much less when applied t,o soils formed of argillaceous earth, basalt, granite, porphyry, clinkstone, and even mountain limestone, because all these contain potass in considerable quantity.'

"Human excrements, on the contrary, are useful in both descriptions of soil, but would be inadequate to supply the silicate of potass which is wanting in the former.

"'The constituents, however, to which the solid excrements of animals in general owe their principal efficacy are the earthy phosphates; and hence we see, why it is that animal manure should favor the growth of corn, which contains so much phosphate of lime and magnesia, and why the earth of bones, and even the ashes of certain kinds of wood, such as the beech, which contain phosphates, may be advantageously substituted, whilst the ashes of others, as of the oak and fir, which are deficient in the phosphates, are of very little avail.

"We see also the cause of the fertilizing quality of liquid manure, as employed in Holland, for those crops which are most subservient to the nourishment of man.

"Liquid manure consists in a great degree of the urine of various animals, which, during its decomposition, exhales a larger quantity of ammonia than any other species of excrement.

"Now all kinds of corn contain nitrogen, and consequently any manure which yields a ready supply of ammonia, must cause a fuller development of those parts of the plant which are of the greatest use to man.

"Even the kind of animal manure usually employed in this country owes its efficacy, so far as it is dependent upon the ammonia present, to the urine, rather than to the solid excrement, of which it is made up, and hence becomes materially deteriorated in this respect, when the more liquid portions are allowed to drain off from it. 
"We may also derive from these considerations, some useful cautions, as to the treatment of this same material.

"Ammonia, in the free or uncombined condition in which it is generated from the decomposition of animal substances, is caustic and noxious to vegetation, and is likewise so volatile that it will escape into the atmosphere so soon as it is produced, unless some means are taken to detain it.

"This causticity is readily removed by promoting its combination with the carbonic acid of the atmosphere, but to prevent its escape during the time necessary for effecting this union, various expedients have been resorted to.

"Where water in sufficient quantity is present, along with the other materials of the dung-heap, this alone will in some measure tend to prevent its volatilization, and the same object is further secured, by admixture with peat, as recommended by Lord Meadowbank, or with sawdust, tanner's bark, turf, and other similar substances. These too are beneficial, not only by moderating the putrefactive process, but also by detaining the ammonia generated within their pores, and thus preventing its loss.

"The advantage of compost heaps, which are strongly advocated by some farmers, depends mainly on these principles.

"The method recommended by a writer, in a late number of the English Agricultural Journal,* to whom a prize of ten sovereigns was awarded for his Essay, consisted, in first making a substratum of peat $\frac{3}{4}$ ths, and sawdust $\frac{1}{4}$ th ; spreading over it the dung from the cattle-sheds, and the urine preserved for the purpose in tanks contiguous; and then, after allowing the mixture to remain exposed for a week, covering it with a fresh layer, nine inches or a foot thick, of peat and sawdust, or of peat alone.

"Several such alternations of peat and manure are to be piled one above the other during the winter, great care being always taken, that the peat should be as dry as possible, by exposing it previously for several months to the weather.

"Now it will be immediately perceived, that these recommendations of a practical farmer completely fulfil the conditions, which theory suggests, for making the best use of our manure, by first neutralizing the ammonia, and afterwards detaining it within the pores of a spongy substance, until it is spread over the land.

"The most effectual plan, however, of preventing its 
loss, would seem to be, not to wait for the slower action of carbonic acid upon it, but to combine it directly with those acids, which form with it salts fixed at common temperatures.

"Hence, Liebig advises the addition of sulphuric or of muriatic acid, both cheap substances, to the other materials of the dung-heap, which, forming with the ammonia present, the sulphates and muriates of that alkali, would at once prevent any loss of it by evaporation.

"If these expedients be not adopted, it should at least be borne in mind, that unless means are taken to prevent it, the most valuable portion of the manure is constantly escaping, during exposure to air and sun, by evaporation, and also by draining off into the ground, whence, instead of a material calculated to afford a ready supply of nitrogen to the plant, we obtain an effete mass, in which that element is in a great measure wanting, and which, therefore, can only influence the growth of plants, by virtue of the phosphoric salts and other fixed ingredients still present in it.

"These views also throw some new light upon the use of gypsum, or sulphate of lime, as a manure to certain crops.

"The fact, that leguminous plants contain this substance as an essential ingredient, may in some measure explain its fertilizing effect on them, but it is also found serviceable to turnips and cabbages, which do not appear to contain it, nor does it seem easy thus to explain the superior advantage said to arise, from scattering it in fine powder over the leaves of clover and saintfoin, as is practised in France and in North America, and with such manifest good effect, that, it is said, if the substance be partially applied to a field, the portions that have received this dressing may afterwards be distinguished from the rest by the superior luxuriance of the crop.

"Liebig, therefore, has suggested another mode in which gypsum may be beneficial to crops in general, by reference to the property which it possesses; of depriving ammonia of its volatility, and thus preventing its escape into the atmosphere.

. "This effect arises from the double decomposition which takes place, when sulphate of lime and carbonate of ammonia are brought together, the lime being converted into a carbonate, and the ammonia uniting with sulphuric acid.

"The above theory of its use being admitted, we may 
be encouraged to extend its application to other crops besides the Leguminosæ, and also to mix it with the dung of our stables, so as to prevent the waste of this valuable material, which is constantly occurring. (See p. 191.)

"But the farmer must be reminded, that it will be necessary, that the sulphate of ammonia resulting from the action of the gypsum, should be brought into contact 'with some substance capable of slowly decomposing it, so as to supply ammonia to the plant.

"For there is no reason to believe, that the organs of a vegetable can decompose sulphate of ammonia, and if they were able so to do, the disengagement of free sulphuric acid in consequence could hardly fail to be injurious to their structure.

"Now a soil consisting of pure sand, or of clay, would be incapable of acting upon this salt, but contradictory as it may seem to the fact, that carbonate of ammonia is decomposed by sulphate of lime, carbonate of lime does appear in a slight degree to disengage ammonia even in the cold, as may be seen by the change of color produced in a piece of turmeric or reddened litmus paper, placed over a vessel containing powdered chalk, as soon as it is moistened with a solution of sulphate of ammonia.

"And since this interchange of constituents is effected rapidly under the influence of a high temperature, as happens in the common method of obtaining carbonate of ammonia artificially by double decomposition, it is worth inquiry, whether it may not be favored likewise by exposure to solar heat and light.

"Where calcareous matter, therefore, exists in the soil, ammonia may be slowly supplied in this manner to the growing plant, and it is possible even, that the carbonate of lime, which seems to be generally present in the sap, may act in the same manner.

"In this way we may readily explain the use of scattering gypsum over the leaves of clover shortly before a shower of rain. The ammonia present in the latter is thus detained, and converted into sulphate by the action of the gypsum upon it, and when introduced into the system by the absorbing surfaces of the plant, it may be again converted into carbonate, by the slow action of the carbonate of lime present in the sap.

"When, however, a more rapid disengagement of ammoniacal gas is required for the nutrition of the intended crop, we ought not to trust to the slow action of carbonate 
of lime, but should apply quicklime to the spots over which the manure has been scattered.

"It is probably in part by setting at liberty the volatile alkali imprisoned in the soil, that quicklime acts so beneficially in agriculture, and in particular, that it improves soil containing a free acid, such as peat earth ; for, independently of its use in neutralizing a substance, which checks vegetation by its antiseptic properties, quicklime may also disengage a portion of ammonia combined with this acid, and thus afford to the plant a more abundant supply of the nitrogen, which it requires.

"Chloride of calcium, common salt, sulphuric and muriatic acids, phosphate of lime, and other salts, may, it would seem, on the principles laid down, be substituted, when gypsum cannot be obtained.

"The chlorides, indeed, like certain oxides, (such as water and carbonic acid,) seem to be decomposed by the plant under the influence of light, for chlorine is exhaled by vegetables near the sea, as oxygen is in other situations. Hence, if muriate of ammonia should result from the action of common salt upon the carbonate of ammonia present in rain, it may undergo decomposition when absorbed by the plant, and contribute in consequence to supply it with nitrogen.

"The above considerations may suggest to us the utility in agriculture of ammoniacal compounds of all kinds, as substitutes for animal manure.

"Sal ammoniac is probably too expensive an article to be employed; but sulphate of ammonia may be had of the wholesale chemist at a price considerably more reasonable, namely, at $22 l$. per ton; and the ammoniacal liquor, which is afforded in abundance by our gas manufactories, through the distillation of coal, is a still cheaper commodity.

"The latter consists principally of carbonate of ammonia, mixed with a certain proportion of the hydro-sulphuret, and, until its use in agriculture was discovered, much of it was allowed to run waste into the Thames, where its noxious qualities destroyed the fish, and rendered the water unpalatable and disgusting.

"Its efficacy as a manure is vouched for by many who have made trial of it upon their land,* and although the hydro-sulphuret of ammonia in a concentrated form would doubtless be fatal to vegetation, yet in a proper state of

" * See a communication by Mr. Paynter, on Gas-water as a Manure, Eng. Agricult. Journ. No. 1, p. 4." 
dilution it may be of service to certain crops, not merely by virtue of the ammonia, but also in consequence of the sulphuretted hydrogen, which it contains, since the latter is found to be an ingredient in the turnip, and in some other tribes of cruciferous plants.

"Where, however, it is found troublesome to preserve, or difficult to convey to a distance this volatile material, an easy method presents itself for retaining for any length of time the ammonia present in it.

"This is done, by availing ourselves of the same principle which has been already explained to you, in treating of the uses of gypsum as a manure ; for as the gas liquor consists of ammonia, combined principally with carbonic acid, it is evident, that it may be converted into a sulphate by admixture with sulphate of lime.

"I am indebted to an excellent scientific chemist* for the following details, which may be of use to the agriculturist in enabling him to appreciate the importance of this commodity, and to prepare for himself any quantity that he may require for his farm.

"One gallon of the ammoniacal liquor added to $1 \mathrm{lb} .2 \frac{1}{2}$ ozs. of powdered but not calcined gypsum, will produce $1 \mathrm{lb}$. of crystallized sulphate of ammonia. To effect the decomposition, the materials should be mixed and stirred up together for ten or twelve hours, a heat, below that of ebullition, being at the same time employed. The sulphate of ammonia remains in solution, and may be obtained in a solid state, by evaporating at a low temperature.

"Theory would suggest, that this material ought to supply nitrogen to the crop at a much cheaper rate than the nitrátes employed for that purpose. For let us suppose, that the farmer wishes to add to his land $60 \mathrm{lbs}$. of crystallized sulphate of ammonia. This may be obtained by introducing about $70 \mathrm{lbs}$. of powdered gypsum uncalcined into 50 gallons of ammoniacal liquor; for my informant found, that one gallon mixed with chloride of calcium yielded 4800 grs. of carbonate of lime, equivalent to about $7200 \mathrm{grs}$. of crystallized sulphate of ammonia, or $1 \mathrm{lb} .3$ ozs. Now 4800 grs. of carbonate of lime are equivalent to $82.50 \mathrm{grs}$, , or to $1 \mathrm{lb} .5 \mathrm{ozs}$. of sulphate of lime, with 2 atoms of water.

"This, therefore, is the quantity of gypsum required, to

" * Mr. Richard Phillips, the superintendent of the chemical department of the establishment, connected with the Museum of Economic Geology, lately instituted by government." 
convert the contents of 1 gallon of gas liquor into sulphate of ammonia, and accordingly, 50 gallons will require 70 lbs. of gypsum, and will produce about $60 \mathrm{lbs}$. of the ammoniacal sulphate.

" Now since the price per ton of gypsum is from $2 l$. to $3 l$., the cost of $70 \mathrm{lbs}$. of it cannot exceed $2 s$., and the labor of mixing the materials may be reckoned at about as much more ; so that to a gas company, where this liquor, not being employed for manufacturing any of the salts of ammonia, has hitherto been regarded as so much refuse, and where the heat requisite for evaporating and crystallizing the product can be obtained with scarcely any increased expenditure, the cost of the impure sulphate would not exceed one penny per pound.

"This then is less than half the cost of an equal quantity of nitrate of soda, which at its present price (23s. per cwt.) may be reckoned at two-pence-halfpenny a pound, and yet it may be shown, that a given weight of sulphate of ammonia contains more ammonia, and consequently ought to yield more nitrogen, than nitrate of soda.*

"Sulphate of ammonia 75 pts. contain of ammonia $17=$ nitrogen 14. whilst

Nitrate of soda ...8 86 pts. . . . . . . 17 = . . 14 .

"So far as theory goes, therefore, the balance would seem to be in favor of the efficiency of sulphate of ammonia over nitrate of soda, in the proportion of 75 to 86 .

"These considerations are merely offered, by way of encouragement to those who may be disposed to make trial of this promising kind of manure, and of course will go for little until they have been tested by experiment.

"There are other materials also employed as manure, which appear to owe their efficacy to the presence of ammonia, - such, for example, as soot, which contains a considerable proportion of this principle united with carbonic acid, and which accordingly has for a long time been advantageously employed as a top-dressing to land.

"Lastly, the foregoing considerations point out the decided superiority of human to other sorts of animal manure.

"Independently of its being richer in most of those ingredients on which the fertilizing property of manure depends, the following circumstance gives it an advantage.

" * Nitrate of potass ought to contain ten per cent. less nitric acid than nitrate of soda, but, as it is a less deliquescent salt, the difference between the two, as obtained in commerce, is not very considerable." 
"When the excrements of the horse or ox are employed, we are obliged to allow of their undergoing a long previous process of fermentation, by which a large proportion of their valuable matter is got rid of, in order, as much as possible, to destroy the vitality of the seeds, which pass undigested along with the fæces. And after all many still remain, and are thus introduced into the fields when the manure is scattered over them.

"By the use of night-soil we avoid this inconvenience, and hence it is, that in China, where it is exclusively employed, the corn-fields are remarkably exempt from weeds.

"Chemistry has suggested means for destroying those offensive qualities which have hitherto limited the use of this species of manure, although it is stated by Liebig, that the method adopted for that purpose on the Continent is defective, inasmuch as a large proportion of their ammoniacal contents is allowed to escape.

"Even under its present management, however, the process may be regarded as one of the most important presents which chemistry has yet made to the practical farmer, by rendering the accumulated filth of a large capital available for his purposes, in the remotest corner of the British empire."

Professor Daubeny concludes his lecture with some highly ingenious speculations on the primary source of the carbon and nitrogen present in plants and animals. He does not deem it probable that a quantity of organic matter was called into existence at once, sufficient to supply the whole of the succeeding races of plants and animals with these ingredients; or that the whole, which is now condensed in the organization of the animal and vegetable kingdoms, was at any one time present in the atmosphere; but that the carbon and nitrogen of plants was originally supplied from the interior of the earth by volcanos. The fertility of the neighborhood of Naples Dr. D. attributes to volcanic exhalations.

"Once grant," he continues, "with Liebig, that the nitrogen, which plants possess, can only be obtained by them through the decomposition of ammonia, and it will follow, that unless this gas be supplied from the interior of the globe, the quantity of organic matter, into which this principle enters as a component part, will be undergoing a continual diminution.

"For we know of no natural processes taking place on the surface of the globe, which generate ammonia, ex- 
cepting those connected with animal and vegetable decomposition; whilst there are many, such as the combustion of various organic substances, which, by resolving bodies containing nitrogen into their constituent elements, would have diminished the aggregate amount of them which might have formerly existed.

"Some compensating process, therefore, is clearly required, and that, if I mistake not, is the disengagement of ammoniacal gas from the interior of the globe."

"Granting, then, what upon Liebig's principles seems most consistent with analogy, namely, that the ammonia, no less than the carbonic acid, which formed the food of the first plants, has been produced, not by processes of animal decay, but by such as were proceeding within the globe prior to the creation of living beings, the notion of a slow and continuous disengagement of both compounds, from the earliest period to the present time, will be received perhaps, as at least the most probable mode of accounting for their unfailing supply.

"Whilst it relieves us from the difficulty of supposing the atmosphere surcharged with these gases at any one period, it suggests to us, at the same time, sublime and interesting views of the arrangements of the Deity, in thus having made all things subservient to one common end, and having ordained, that the mighty agents of destruction, which exist in the bowels of the earth, should minister, like the malignant Genii of some eastern fable, to the wants and necessities of the living beings, which He has placed upon its surface."

\section{USE OF PHOSPHATE OF SODA IN CALICO PRINTING.*}

(See page 186.)

The discovery of the principle which led to the use of phosphate of soda, was made in the United States, by Dr. Dana, of Lowell. The first practical application of the salt was made, in consequence of Dr. Dana's researches, by Mr. J. D. Prince, Jr., at the works of the Merrimack Manufacturing Company in Lowell, in 1834. Mr. J. D. Prince, Sen., the scientific and accomplished superintendent of the establishment, was engaged with Dr. Dana for a

* Substance of a communication from Dr. Dana. 
series of years on this subject. In 1839, Mr. Prince, Jr. carried the process to England, and, with Mr. J. Mercer and Blyth, took out letters patent. Mr. Prince sold his right to Messrs. Mercer and Blyth, who introduced the process into the establishments on the Continent. The article is now made by M. Kestner, of Thann, who observes, in his letter to the "Société Industrielle de Mulhouse," accompanying a sample; and on which their committee reported, Bulletin No. 6.3, that "the article is the invention of Messrs. Mercer and Blyth, printers of calicoes near Manchester."

Dr. Liebig probably, derived his knowledge of this improvement from the Bulletin referred to above, and his statement is only partial respecting the effects of cow-dung. The discovery of the principle of its action has led to the employment of other salts, which produce effects equally good as phosphates.

\section{DANIELL'S ARTIFICIAL MANURE.*}

THE basis of this manure is wood reduced to powder, sawdust, which is to be thoroughly saturated with bituminous and animal matters of all or any kind; to this is to be added small proportions of soda and quicklime. The sample exhibited to the Royal Agricultural Society, was a coarse black powder, having a strong smell, somewhat resembling coal tar. In Fngland its price will be about one third that of bone dust. It is a kind of artificial bituminous coal. It should be buried two or three inches under the surface of the soil. For grass land, it is to be well mixed with a considerable portion of ordinary unvalued mould. The quantity to be used will vary with the crop. About twenty-four bushels per acre are recommended for wheat, and half as much more, or thirty-six bushels, may be carefully applied for turnips or mangel-wurtzel. Its direct effect is thought to be the conveyance to the soil of the direct nutriment of future growth. This effect is produced by the supply of ammonia to the soil in substances calculated to retain it for a time, - to again absorb it from the atmosphere, - as they give it out to plants during their growth. It will probably prevent also the ravages of insects.

* Abridged from notices in the New Genesee Farmer, Vol. III., by J. E. T. 


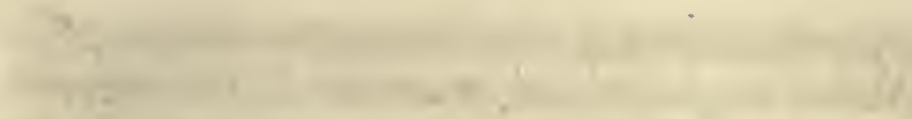

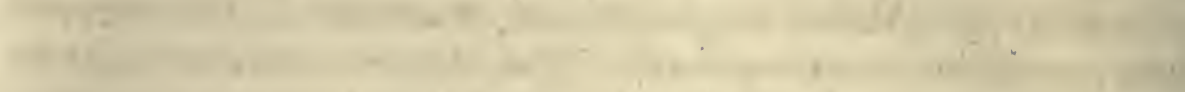

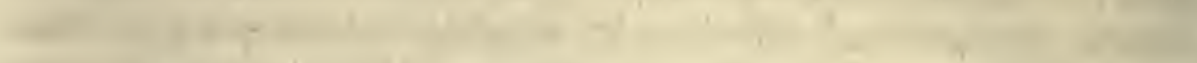

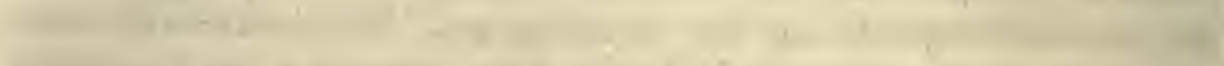

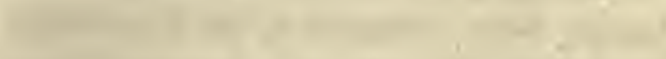

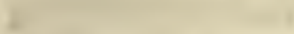

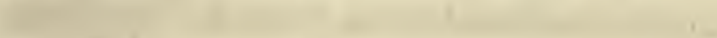

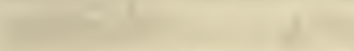

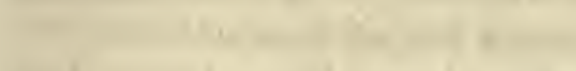

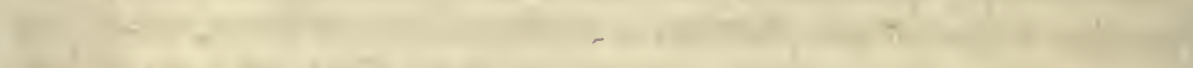

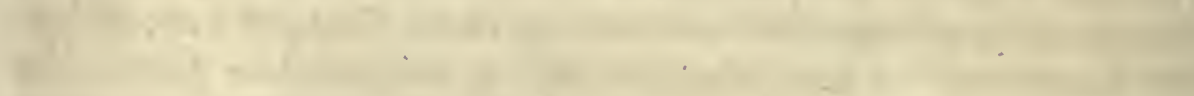
$-1$

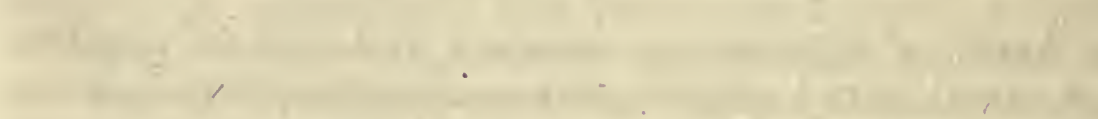

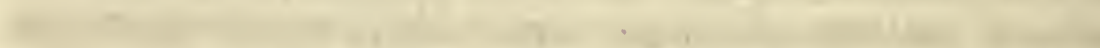

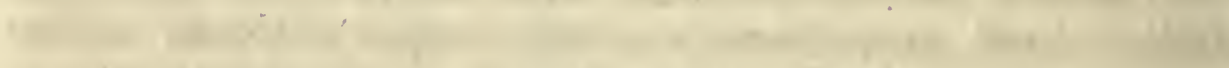

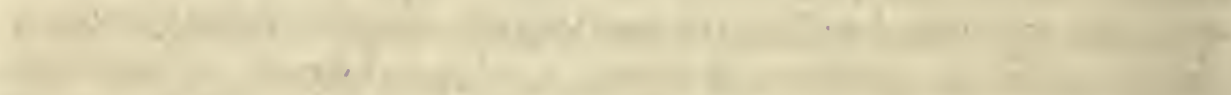<smiles>C[C+](C)C1CC1</smiles>

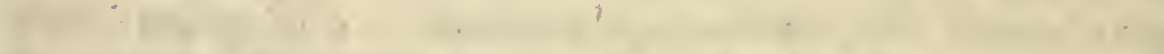

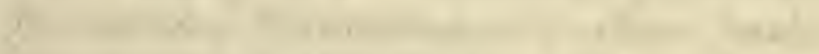

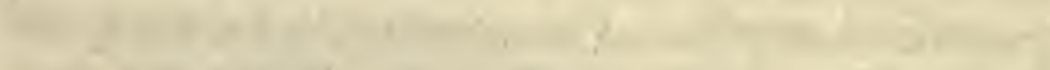

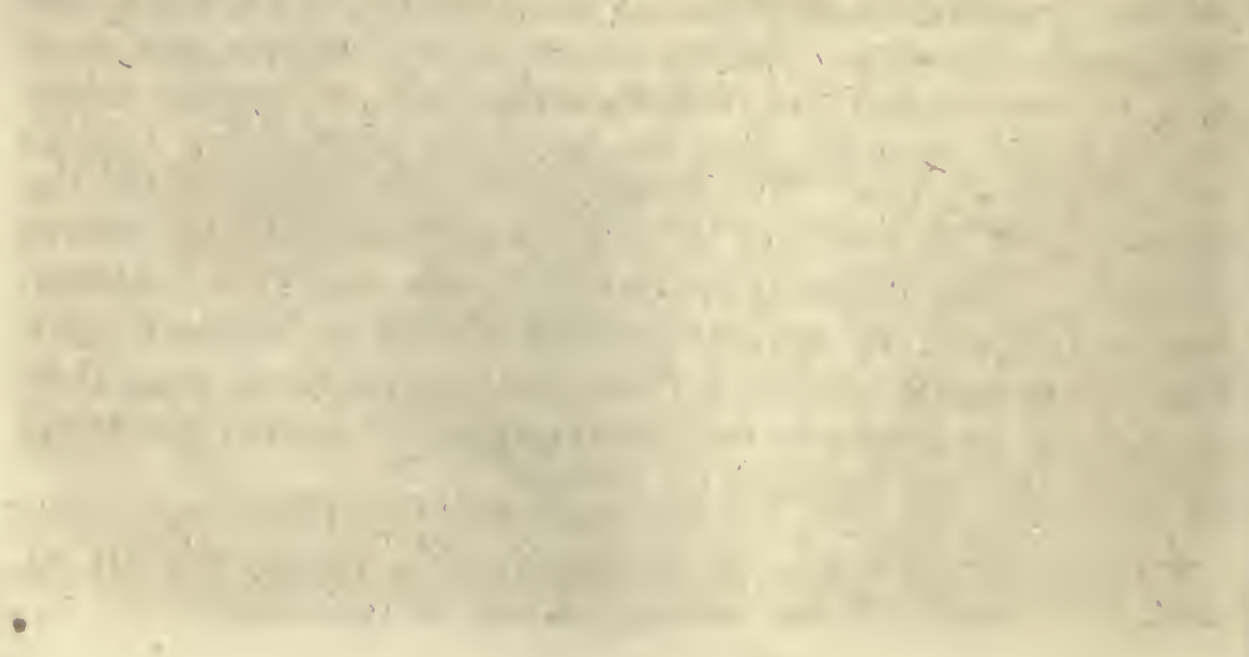




\section{P A R T II.}

OF THE CHEMICAL PROCESSES OF FERMENTATION, DECAY, AND PUTREFACTION.

\section{CHAPTER I.}

\section{CHEMICAL TRANSFORMATIONS.}

Woopy fibre, sugar, gum, and all such organic compounds, suffer certain changes when in contact with other bodies; that is, they suffer decomposition.

There are two distinct modes in which these decompositions take place in organic chemistry.

When a substance composed of two compound bodies, crystallized oxalic acid for example, is brought in contact with concentrated sulphuric acid, a complete decomposition is effected upon the application of a gentle heat. Now crystallized oxalic acid is a combination of water with the anhydrous acid; but concentrated sulphuric acid possesses a much greater affinity for water than oxalic acid, so that it attracts all the water of crystallization from that substance. In consequence of this abstraction of the water, anhydrous oxalic acid is set free; but as this acid cannot exist in a free state, a division of its constituents necessarily ensues, by which carbonic acid and carbonic oxide are produced, and evolved in the gaseous form in equal volumes. In this example, the decomposition is the consequence of the removal of two constituents (the elements of water), which unite with the sulphuric acid, and its cause is the superior affinity of the acting body (the sulphuric acid) for water. In consequence of the removal of 
the component parts of water, the remaining elements enter into a new form; in place of oxalic acid, we have its elements in the form of carbonic acid and carbonic oxide.

This form of decomposition, in which the change is effected by the agency of a body which unites with one or more of the constituents of a compound, is quite analogous to the decomposition of inorganic substances. When we bring sulphuric acid and nitrate of potash together, nitric acid is separated in. consequence of the affinity of sulphuric acid for potash; in consequence, therefore, of the formation of a new compound (sulphate of potash).

In the second form of these decompositions, the chemical affinity of the acting body causes the component parts of the body which is decomposed to combine so as to form new compounds, of which either both, or cnly one, combine with the acting body. Let us take dry wood, for example, and moisten it with sulphuric acid; after a short time the wood is carbonized, while the sulphuric acid remains unchanged, with the exception of its being united with more water than it possessed before. Now this water did not exist as such in the wood, although its elements, oxygen and hydrogen, were present; but by the chemical attraction of sulphuric acid for water, they were in a certain measure compelled to unite in this form; and in consequence of this, the carbon of wood was separated as charcoal.

Hydrocyanic acid* and water, in contact with hydrochloric acid, $\dagger$ are mutually decomposed. The nitrogen of the hydrocyanic acid, and a certain quantity of the hydrogen of the water, unite together and form ammonia; whilst the carbon and hydrogen of the hydrocyanic acid combine with the oxygen of the water, and form formic acid. $\ddagger$ The ammonia com-

\footnotetext{
* See page 70, note.

$\uparrow$ Formerly called Muriatic Acid, ohtained from sea salt and compos. ed of Hydrogen and Chlorine in equal vols. $\mathrm{H}+\mathrm{Cl}$.

$\ddagger$ See page 70 .
} 
bines with the muriatic acid. Here the contact of muriatic acid with water and hydrocyanic acid causes a disturbance in the attraction of the elements of both compounds, in consequence of which they arrange themselves into new combinations, one of which, - ammonia, - possesses the power of uniting with the acting body.

Inorganic chemistry can present instances analogous to this aclass of decomposition also; but there are forms of organic chemical decomposition of a very different kind, in which none of the component parts of the matter which suffers decomposition enter into combination with the body which determines the decomposition. In cases of this kind a disturbance is produced in the mutual attraction of the elements of a compound, and they in consequence arrange themselves into one or several new combinations, which are incapable of suffering further change under the same conditions.

When, by means of the chemical affinity of a second body, by the influence of heat, or through any other causes, the composition of an organic compound is made to undergo such a change, that its elements form two or more new compounds, this manner of decomposition is called a chemical transformation or metamorphosis. - It is an essential character of chemical transformations, that none of the elements of the body decomposed are singly set at liberty.

The changes, which are designated by the terms fermentation, decay, and putrefaction, are chemical transformations effected by an agency which has hitherto escaped attention, but the existence of which will be proved in the following pages. 


\section{CHAPTER II.}

ON THE CAUSES WHICH EFFECT FERMENTATION, DECAY,* AND PUTREFAC'TION.

Attention has been recently directed to the fact, that a body in the act of combination or decomposition exercises an influence upon any other body with which it may be in contact. Platinum, for example, does not decompose nitric acid; it may be boiled with this acid without being oxidized by it, even when in a state of such fine division, that it no longer reflects light (black spongy platinum). But an alloy of silver and platinum dissolves with great ease in nitric acid; the oxidation which the silver suffers, causes the platinum to submit to the same change; or, in other words, the latter body, from its contact with the oxidizing silver, acquires the property of decomposing nitric acid.

Copper does not decompose water, even when boiled in dilute sulphuric acid; but an alloy of copper, zinc, and nickel, dissolves easily in this acid with evolution of hydrogen gas.

Tin decomposes nitric acid with great facility, but water with difficulty; and yet, when tin is dissolved in nitric acid, hydrogen is evolved at the same time, from a decomposition of the water contained in the acid, and ammonia is formed in addition to oxide of tin.

In the examples here given, the only combination or decomposition which can be explained by chemical affinity is the last. In the other cases, electrical

* An essential distinction is drawn in the following part of the work, between decay and putrefuction (Verwesung und Faulniss), and they are shown to depend on different causes; but as the word decay is not generally applied to a distinct species of decomposition, and does not indicate its true nature, I shall in future, at the suggestion of the author, employ the term eremacuusis, the meaning of which has been already explained. - ED. 
action ought to have retarded or prevented the oxidation of the platinum or copper while they were in contact with silver or zinc, but, as experience shows, the influence of the opposite electrical conditions is more than counterbalanced by chemical actions.

The same phenomena are seen in a less dubious form in compounds, the elements of which are held together only by a feeble affinity. It is well known, that there are chemical compounds of so unstable a nature, that changes in temperature and electrical condition, or even simple mechanical friction, or contact with bodies of apparently totally indifferent natures, cause such a disturbance in the attraction of their constituents, that the latter enter into new forms, without any of them combining with the acting body. These compounds appear to stand but just within the limits of chemical combination, and agents exercise a powerful influence on them, which are completely devoid of action on compounds of a stronger affinity. Thus, by a slight increase of temperature, the elements of hypochlorous acid* separate from one another with evolution of heat and light; chloride of nitrogen explodes by contact with many bodies, which combine neither with chlorine nor nitrogen at common temperatures; and the contact of any solid substance is sufficient to cause the explosion of iodide of nitrogen, or fulminating silver.

It has never been supposed that the causes of the decomposition of these bodies should be ascribed to a peculiar power, different from that which regulates chemical affinity, - a power which mere contact with the down of a feather is sufficient to set in activity, and which, once in action, gives rise to the decomposition. These substances have always been viewed as chemical compounds of a very unstable nature, in which the component parts are in a state of such tension, that the least disturbance overcomes their chemical affinity. They exist only by the vis inertia,

* Formerly, protoxide of chlorine. 
and any shock or movement is sufficient to destroy the attraction of their component parts, and consequently their existence in their definite form.

Peroxide of hydrogen* belongs to this class of bodies; it is decomposed by all substances capable of attracting oxygen from it, and even by contact with many bodies, such as platinum or silver, which do not enter into combination with any of its constituents. In this respect, its decomposition depends evidently upon the same causes which effect that of iodide of nitrogen, or fulminating silver. Yet it is singular, that the cause of the sudden separation of the component parts of peroxide of hydrogen has been viewed as different from those of common decomposition, and has been ascribed to a new power termed the catalytic force. Now, it has not been considered, that the presence of the platinum and silver serves here only to accelerate the decomposition; for without the contact of these metals, the peroxide of hydrogen decomposes spontaneously, although very slowly. The sudden separation of the constituents of peroxide of hydrogen differs from the decomposition of gaseous hypochlorous acid, or solid iodide of nitrogen, only in so far as the decomposition takes place in a liquid.

A remarkable action of peroxide of hydrogen has attracted much attention, because it differs from ordinary chemical phenomena. This is the reduction which certain oxides suffer by contact with this substance, on the instant at which the oxygen separates from the water. The oxides thus easily reduced, are those of which the whole, or part at least, of their oxygen is retained merely by a feeble affinity, such as the oxides of silver and of gold, and peroxide of lead.

Now, other oxides, which are very stable in composition, effect the decomposition of peroxide of hy-

* A remarkable compound, consisting of 1 Hydrogen, and 2 Oxygen. See description and process for obtaining, in WEBSTER's Chemistry, p. 134 . 
drogen, without experiencing the smallest change; but when oxide of silver is employed to effect the decomposition, all the oxygen of the silver is carried away with that evolyed from the peroxide of hydrogen, and, as a result of the decomposition, water and metallic silver remain. When peroxide of lead* is used for the same purpose, half its oxygen escapes as a gas. Peroxide of manganese may in the same manner be reduced to the protoxide, and ogygen set at liberty, if an acid is at the same time present, which will exercise an affinity for the protoxide and convert it into a soluble salt. If, for example, we add to peroxide of hydrogen sulphuric acid, and then peroxide of manganese in the state of fine powder, much more oxygen is evolved than the compound of oxygen and hydrogen could yield; and if we examine the solution which remains, we find a salt of the protoxide of manganese, so that half of the oxygen has been evolved from the peroxide of that metal.

A similar phenomenon occurs, when carbonate of silver is treated with several organic acids. Pyruvic acid, for example, combines readily with pure oxide of silver, and forms a salt of sparing solubility in water. But when this acid is brought in contact with carbonate of silver, the oxygen of part of the oxide escapes with the carbonic acid, and metallic silver remains in the state of a black powder. (Berzelius.)

Now no other explanation of these phenomena can, be given, than that a body in the act of combination or decomposition enables another body, with which it is in contact, to enter into the same state. It is evident that the active state of the atoms of one body has an influence upon the atoms of a body in contact with it; and if these atoms are capable of the same change as the former, they likewise under-

* A peroxide is one that contains the largest proportion of oxygen. When several compounds of metals and oxygen occur, that which contains the smallest proportion of oxygen is called the first or protoxide. 
go that change; and combinations and decompositions are the consequence. But when the atoms of the second body are not capable of such an action, any further disposition to change ceases from the moment at which the atoms of the first body assume the state of rest, that is, when the changes or transformations of this body are quite completed.

This influence exerted by one compound upon the other, is exactly similar to that which a body in the act of combustion exercises upon a combustible body in its vicinity; with this difference only, that the causes which determine the participation and duration of these conditions are different. For the cause, in the case of the combustible body, is heat, which is generated every moment anew; whilst in the phenomena of decomposition and combination which we are considering at present, the cause is a body in the state of chemical action, which exerts the decomposing influence only so long as this action continues.

Numerous facts show, that motion alone exercises a considerable influence on chemical forces. Thus, the power of cohesion does not act in many saline solutions, even when they are fully saturated with salts, if they are permitted to cool whilst at rest. In such a case, the salt dissolved in a liquid does not crystallize; but when a grain of sand is thrown into the solution, or when it receives the slightest movement, the whole liquid becomes suddenly solid while heat is evolved. The same phenomenon happens with water, for this liquid may be cooled much under $32^{\circ} \mathrm{F} .\left(0^{\circ}\right.$ C. $)$, if kept completely undisturbed, but solidifies in a moment when put in motion.

The atoms of a body must in fact be set in motion before they can overcome the vis inertia so as to arrange themselves into certain forms. A dilute solution of a salt of potash mixed with tartaric acid yields no precipitate whilst at rest; but if motion is communicated to the solution by agitating it briskly, solid crystals of cream of tartar are deposited. A solu- 
tion of a salt of magnesia, also, which is not rendered turbid by the addition of phosphate of ammonia, deposits the phosphate of magnesia and ammonia on those parts of the vessel touched with the rod employed in stirring.

In the processes of combination and decomposition under consideration, motion, by overcoming the vis inertia, gives rise immediately to another arrangement of the atoms of a body, that is, to the production of a compound which did not before exist in it. Of course these atoms must previously possess the power of arranging themselves in a certain order, otherwise both friction and motion would be without the smallest influence.

'The simple permanence in position of the atoms of a body, is the reason that so many compounds appear to present themselves, in conditions, and with properties, different from those which they possess, when they obey the natural attractions of their atoms. Thus sugar and glass, when melted and cooled rapidly, are transparent, of a conchoidal fracture, and elastic and flexible to a certain degree. But the former becomes dull and opaque on keeping, and exhibits crystalline faces by cleavage, which belong. to crystallized sugar. Glass assumes also the same condition, when kept soft by heat for a long period; it becomes white, opaque, and so hard as to strike fire with steel. Now, in both these bodies, the compound molecules evidently have different positions. in the two forms. In the first form their attraction did not act in the direction in which their power of cohesion was strongest. It is known, also, that when sulphur is melted and cooled rapidly by throwing it into cold water, it remains transparent, elastic, and: so soft that it may be drawn out into long threads; but that after a few hours or days, it becomes again hard and crystalline.

The remarkable fact here is, that the amorphous sugar or sulphur returns again into the crystalline condition, without any assistance from an exterior 
cause; a fact which shows, that their molecules have assumed another position, and that they possess, therefore, a certain degree of mobility, even in the condition of a solid. A very rapid transposition or transformation of this kind is seen in arragonite, a mineral which possesses exactly the same composition as calcareous spar, but of which the hardness and crystalline form prove that its molecules are arranged in a different manner. When a crystal of arragonite is heated, an interior motion of its molecules is caused by the expansion; the permanence of their arrangement is destroyed; and the crystal splinters with much violence, and falls into a heap of small crystals of calcareous spar.

It is impossible for us to be deceived regarding the causes of these changes. They are owing to a disturbance of the state of the equilibrium, in consequence of which the particles of the body put in motion obey other affinities or their own natural attractions.

But if it is true, as we have just shown it to be, that mechanical motion is sufficient to cause a change of condition in many bodies, it cannot be doubted that a body in the act of combination or decomposition is capable of imparting the same condition of motion or activity in which its atoms are to certain other bodies: or in other words, to enable other bodies with which it is in contact to enter into combinations, or suffer decompositions.

The reality of this influence has been already sufficiently proved by the facts derived from inorganic chemistry, but it is of much more frequent occurrence in the relations of organic matter, and causes very striking and wonderful phenomena.

By the terms fermentation, putrefaction, and eremacausis, are meant those changes in form and properties which compound organic substances undergo when separated from the organism, and exposed to the influence of water and a certain temperature. Fermentation and putrefaction are examples of that 
kind of decomposition, which we have named transformations: the elements of the bodies capable of undergoing these changes arrange themselves into new combinations, in which the constituents of water generally take a part.

Eremacausis (or decay) differs from fermentation and putrefaction, inasmuch as it cannot take place without the access of air, the oxygen of which is absorbed by the decaying bodies. Hence, it is a process of slow combustion, in which heat is uniformly evolved, and occasionally even light. In the processes of decomposition termed fermentation and putrefaction, gaseous products are very frequently formed, which are either inodorous, or possess a very offensive smell.

The transformations of those matters which evolve gaseous products without odor, are now, by pretty general consent, designated by the term fermentation; whilst to the spontaneous decomposition of bodies which emit gases of a disagreeable smell, the term putrefaction is applied. But the smell is of course no distinctive character of the nature of the decomposition, for both fermentation and putrefaction are processes of decomposition of a similar kind, the one of substances destitute of nitrogen, the other of substances which contain it.

It has also been customary to distinguish from fermentation and putrefaction a particular class of transformations, viz., those in which conversions and transpositions are effected without the evolution of gaseous products. But the conditions under which the products of the decomposition present themselves are purely accidental; there is, therefore, no reason for the distinction just mentioned. 
cause; a fact which shows, that their molecules have assumed another position, and that they possess, therefore, a certain degree of mobility, even in the condition of a solid. A very rapid transposition or transformation of this kind is seen in arragonite, a mineral which possesses exactly the same composition as calcareous spar, but of which the hardness and crystalline form prove that its molecules are arranged in a different manner. When a crystal of arragonite is heated, an interior motion of its molecules is caused by the expansion; the permanence of their arrangement is destroyed; and the crystal splinters with much violence, and falls into a heap of small crystals of calcareous spar.

It is impossible for us to be deceived regarding the causes of these changes. They are owing to a disturbance of the state of the equilibrium, in consequence of which the particles of the body put in motion obey other affinities or their own natural attractions.

But if it is true, as we have just shown it to be, that mechanical motion is sufficient to cause a change of condition in many bodies, it cannot be doubted that a body in the act of combination or decomposition is capable of imparting the same condition of motion or activity in which its atoms are to certain other bodies: or in other words, to enable other bodies with which it is in contact to enter into combinations, or suffer decompositions.

The reality of this influence has been already suffieiently proved by the facts derived from inorganic chemistry, but it is of much more frequent occurrence in the relations of organic matter, and causes very striking and wonderful phenomena.

By the terms fermentation, putrefaction, and eremacausis, are meant those changes in form and properties which compound organic substances undergo when separated from the organism, and exposed to the influence of water and a certain temperature. Fermentation and putrefaction are examples of that 
kind of decomposition, which we have named transformations: the elements of the bodies capable of undergoing these changes arrange themselves into new combinations, in which the constituents of water generally take a part.

Eremacausis (or decay) differs from fermentation and putrefaction, inasmuch as it cannot take place without the access of air, the oxygen of which is absorbed by the decaying bodies. Hence, it is a process of slow combustion, in which heat is uniformly evolved, and occasionally even light. In the processes of decomposition termed fermentation and putrefaction, gaseous products are very frequently formed, which are either inodorous, or possess a very offensive smell.

The transformations of those matters which evolve gaseous products without odor, are now, by pretty general consent, designated by the term fermentation; whilst to the spontaneous decomposition of bodies which emit gases of a disagreeable smell, the term putrefaction is applied. But the smell is of course no distinctive character of the nature of the decomposition, for both fermentation and putrefaction are processes of decomposition of a similar kind, the one of substances destitute of nitrogen, the other of substances which contain it.

It has also been customary to distinguish from fermentation and putrefaction a particular class of transformations, viz., those in which conversions and transpositions are effected without the evolution of gaseous products. But the conditions under which the products of the decomposition present themselves are purely accidental; there is, therefore, no reason for the distinction just mentioned. 


\section{CHAPTER III.}

\section{FERMENTATION AND PUTREFACTION.}

Several bodies appear to enter spontaneously into the states of fermentation and putrefaction, particularly such as contain nitrogen or azotized substances. Now, it is very remarkable, that very small quantities of these substances, in a state of fermentation or putrefaction, possess the power of causing unlimited quantities of similar matters to pass into the same state. Thus, a small quantity of the juice of grapes in the act of fermentation, added to a large quantity of the same fluid, which does not ferment, induces the state of fermentation in the whole mass. So likewise the most minute portion of milk, paste, juice of the beet-root, flesh, or blood, in the state of putrefaction, causes fresh milk, paste, juice of the beet-root, flesh, or blood, to pass into the same condition when in contact with them.

These changes evidently differ from the class of common decompositions which are effected by chemical affinity; they are chemical actions, conversions, or decompositions, excited by contact with bodies already in the same condition. In order to form a clear idea of these processes, analogous and less complicated phenomena must previously be studied.

The compound nature of the molecules of an organic body, and the phenomena presented by them when in relation with other matters, point out the true cause of these transformations. Evidence is afforded even by simple bodies, that in the formation of combinations, the force with which the combining elements adhere to one another is inversely proportional to the number of simple atoms in the compound molecule. Thus, protoxide of manganese by absorption of oxygen is converted into the sesquioxide, the peroxide, manganic, and hypermanganic 
acids, the number of atoms of oxygen being augmented by $\frac{1}{2}$, by 1 , by 2 , and by 5 . But all the oxygen contained in these compounds, beyond that which belongs to the protoxide, is bound to the manganese by a much more feeble affinity; a red heat causes an evolution of oxygen from the peroxide, and the manganic and hypermanganic acids cannot be separated from their bases without undergoing immediate decomposition.

There are many facts which prove, that the most simple inorganic compounds are also the most stable, and undergo decomposition with the greatest difficulty, whilst those which are of a complex composition yield easily to changes and decompositions. The cause of this evidently is, that, in proportion to the number of atoms which enter into a compound, the directions in which their attractions act will be more numerous.

Whatever ideas we may entertain regarding the infinite divisibility of matter in general, the existence of chemical proportions removes every doubt respecting the presence of certain limited groups or masses of matter which we have not the power of dividing. 'The particles of matter called equivalents in chemistry are not infinitely small, for they possess a weight, and are capable of arranging themselves in the most various ways, and of thus forming innumerable compound atoms. The properties of these compound atoms differ in organic nature, not only according to the form, but also in many instances according to the direction and place, which the simple atoms take in the compound molecules.

When we compare the composition of organic compounds. with inorganic, we are quite amazed at the existence of combinations, in one single molecule of which, ninety or several hundred atoms or equiv-. alents are united. Thus, the compound atom of an organic acid of very simple composition, acetic acid, for example, contains twelve equivalents of simple. elements; one atom of kinovic acid contains 33,1 : 
of sugar 36,1 of amygdalin 90 , and 1 of stearic acid 138 equivalents. 'The component parts of animal bodies are infinitely more complex even than these.

Inorganic compounds differ from organic in as great a degree in their other characters as in their simplicity of constitution. Thus, the decomposition of a compound atom of sulphate of potash is aided by numerous causes, such as the power of cohesion, or the capability of its constituents to form solid; insoluble, or at certain temperatures volatile compounds with the body brought into contact with it, and nevertheless a vast number of other substances produce in it not the slightest change. Now, in the decomposition of a complex organic atom, there is nothing similar to this.

The empirical formula of sulphate of potash is $\mathrm{SKO}_{4}$ * It contains only 1 eq. of sulphur, and 1 eq. of potassium. We may suppose the oxygen to be differently distributed in the compound, and by a decomposition we may remove a part or all of it, or replace one of the constituents of the compound by another substance. But we cannot produce a different arrangement of the atoms, because they are already disposed in the simplest form in which it is possible for them to combine. Now, let us compare the composition of sugar of grapes with the above: here 12 eq. of carbon, 12 eq. of hydrogen, and 12 eq. of oxygen, are united together, and we know that they are capable of combining with each other in the most various ways. From the formula of sugar, we might consider it either as a hydrate of carbon, wood, starch, or sugar of milk, or further, as a compound of ether with alcohol or of formic acid with sachulmin. $\uparrow$ Indeed, we may calculate almost all the known organic compounds destitute of nitrogen

* $\mathrm{S}$ denotes sulphur, $\mathrm{K}$ (Kali) potash, $\mathrm{O}$ oxygen, 4 the number of atoms. When no number is used, one atom is understood.

† The black precipitate obtained by the action of hydrochloric acid on sugar. 
from sugar, by simply adding the elements of water, or by replacing any one of its elementary constituents by a different substance. The elements necessary to form these compounds are, therefore, contained in: the sugar, and they must also possess the power of forming numerous combinations amongst themselves by their mutual attractions.

Now, when we examine what changes sugar undergoes when brought into contact with other bodies which exercise a marked influence upon it, we find, that these changes are not confined to any narrow limits, like those of inorganic bodies, but are in fact unlimited.

The elements of sugar yield to every attraction, and to each in a peculiar manner. In inorganic compounds, an acid acts upon a particular constituent of the body, which it decomposes; by virtue of its affinity for that constituent, and never resigns its proper chemical character, in whatever form it may be applied. But when it acts upon sugar, and induces great changes in that compound, it does this not by any superior affinity for a base existing in the sugar, but by disturbing the equilibrium in the mutual attraction of the elements of the sugar amongst themselves. Muriatic and sulphuric acids, which differ so much from one another both in characters and composition, act in the same manner upon sugar. But the action of both varies according to the state in which they are; thus they act in one way when dilute, in another when concentrated, and even differences in their temperature cause a change in their action. Thus sulphuric acid of a moderate degree of concentration converts sugar into a black carbonaceous matter, forming at the same time acetic and formic acids. But when the acid is more diluted, the sugar is converted into two brown substances, both of them containing carbon and the elements of water. Again, when sugar is subjected to the action of alkalies, a whole series of different new products is obtained; while oxidizing agents, such as nitric 
acid, produce from it carbonic acid, acetic acid, oxalic acid, formic acid, and many other products which have not yet been examined.

If, from the facts here stated, we estimate the power with which the elements of sugar are united together, and judge of the force of their attraction by the resistance which they offer to the action of bodies brought into contact with them, we must regard the atom of sugar as belonging to that class of compound atoms, which exist only by the vis. inertia of their elements. Its elements seem merely to retain passively the position and condition in which they had been placed, for we do not observe that they resist a change of this condition by their own mutual attraction, as is the case with sulphate of potash.

Now it is only such combinations as sugar, combinations, therefore, which possess a very complex molecule, which are capable of undergoing the decompositions named fermentation and putrefaction.

We have seen that metals acquire a power, which they do not of themselves possess, namely, that of decomposing water and nitric acid, by simple contact with other metals in the act of chemical combination. We have also seen, that peroxide of hydrogen and the persulphuret of the same element, in the act of decomposition, cause other compounds of a similar kind, but of which the elements are much more strongly combined, to undergo the same decomposition, although they exert no chemical affinity or attraction for them or their constituents. The cause which produces these phenomena will be also recognised, by attentive observation, in those matters which excite fermentation or putrefaction. All bodies in the act of combination or decomposition have the property of inducing those processes; or, in other words, of causing a disturbance of the statical equilibrium in the attractions of the elements of complex organic molecules, in consequence of which 
those elements group themselves anew, according to their special affinities.

The proofs of the existence of this cause of action can be easily produced; they are found in the characters of the bodies which effect fermentation and putrefaction, and in the regularity with which the distribution of the elements takes place in the subsequent transformations. This regularity depends exclusively on the unequal affinity which they possess for each other in an isolated condition. The action of water on wood, charcoal, and cyanogen, the simplest of the compounds of nitrogen, suffices to illustrate the whole of the transformations of organic bodies; of those in which nitrogen is a constituent, and of those in which it is absent.

\section{CHAPTER IV.}

ON THE TRANSFORMATION OF BODIES WHICH DO NOT CONTAIN NITROGEN AS A CONSTITUENT, AND OF THOSE IN WHICH IT IS PRESENT.

When oxygen and hydrogen combined in equal equivalents, as in steam, are conducted over charcoal, heated to the temperature at which it possesses the power to enter into combination with one of these elements, a decomposition of the steam ensues. An oxide of carbon (either carbonic oxide or carbonic acid) is under all circumstances formed, while the hydrogen of the water is liberated, or, if the temperature be sufficient, unites with the carbon, forming carburetted-hydrogen. Accordingly, the carbon is shared between the elements of the water, the oxygen and hydrogen. Now a participation of this kind, but even more complete, is observed in every transformation, whatever be the nature of the causes by which it is effected. 
Acetic and meconic* acids suffer a true transformation under the influence of heat, that is, their component elements are disunited, and form new compounds without any of them being singly disengaged. Acetic acid is converted into acetone and carbonic acid ( $44 \mathrm{H} 3 \mathrm{O} 3=\mathrm{C} 3 \mathrm{H} 3 \mathrm{O}+\mathrm{CO} 2$ ), and meconic acid into carbonic acid and komenic acid; whilst by the influence of a higher temperature, the latter is further decomposed into pyromeconic acid and carbonic acid.

- Now in these cases the carbon of the bodies decomposed is shared between the oxygen and hydrogen; part of it unites with the oxygen and forms carbonic acid, whilst the other portion enters into combination with the hydrogen, and an oxide of a carbo-hydrogen is formed, in which all the hydrogen is contained.

In a similar manner, when alcohol is exposed to a gentle red heat, its carbon is shared between the elements of the water, - an oxide of a carbo-hydrogen which contains all the oxygen, and some gaseous compounds of carbon and hydrogen being produced.

It is evident, that during transformations caused by heat, no foreign affinities can be in play, so that the new compounds must result merely from the elements arranging themselves, according to the degree of their mutual affinities, into new combinations, which are constant and unchangeable in the conditions under which they were originally formed, but undergo changes when these conditions become different. If we compare the products of two bodies, similar in composition but different in properties, which are subjected to transformations by two different causes, we find that the manner in which the atoms are transposed, is absolutely the same in both.

In the transformation of wood in marshy soils, by what we call putrefaction, its carbon is shared

* An acid existing in opium, and named from the Greek for poppy. 
between the oxygen and hydrogen of its own substance, and of the water, - carburetted hydrogen is consequently evolved, as well as carbonic acid, both of which compounds have an analogous composition $(\mathrm{CH} 2, \mathrm{CO} 2) *$ *

Thus also in that transformation of sugar, which is called fermentation, its elements are divided into two portions; the one, carbonic acid, which contains $\frac{2}{3}$ of the oxygen of sugar; and the other, alcohol, which contains all its hydrogen.

In the transformation of acetic acid produced by a red heat, carbonic acid, which contains $\frac{2}{3}$ of the oxygen of the acetic acid, is formed, and acetone, which contains all its hydrogen.

It is evident from these facts, that the elements of a complex compound are left to their special attractions whenever their equilibrium is disturbed, from whatever cause this disturbance may proceed. It appears, also, that the subsequent distribution of the elements, so as to form new. combinations, always takes place in the same way, with this difference only, that the nature of the products formed is dependent upon the number of atoms of the elements which enter into action; or, in other words, that the products differ ad infinitum, according to the composition of the original substance.

ON THE TRANSFORMATION OF BODIES CONTAINING NITROGEN.

When those substances are examined which are most prone to fermentation and putrefaction, it is found that they are all, without exception, bodies which contain nitrogen. In many of these compounds, a transposition of their elements occurs spontaneously as soon as they cease to form a part of a living organism; that is, when they are drawn

* C carbon, $\mathrm{H}$ hydrogen, $\mathrm{O}$ oxygen. 
out of the sphere of attraction in which alone they are able to exist.

There are, indeed, bodies destitute of nitrogen, which possess a certain degree of stability only when in combination, but which are unknown in an isolated condition, because their elements, freed from the power by which they were held together, arrange themselves according to their own natural attractions. Hypermanganic, manganic, and hyposulphurous acids, belong to this class of substances, which however are rare.

The case is very different with azotized bodies. It would appear that there is some peculiarity in the nature of nitrogen, which gives its compounds the power to decompose spontaneously with so much facility. Now, nitrogen is known to be the most indifferent of all the elements; it evinces no particular attraction to any one of the simple bodies; and this character it preserves in all its combinations, a character which explains the cause of its easy separation from the matters with which it is united.

It is only when the quantity of nitrogen exceeds a certain limit, that azotized compounds have some degree of permanence, as is the case with melamin, ammelin, \&c. Their liability to change is also diminished, when the quantity of nitrogen is very small in proportion to that of the other elements with which it is united, so that their mutual attractions preponderate.

This easy transposition of atoms is best seen in the fulminating silvers, in fulminating mercury, in the iodide or chloride of nitrogen, and in all fulminating compounds.

All other azotized substances acquire the same power of decomposition, when the elements of water are brought into play; and indeed, the greater part of them are not capable of transformation, while this necessary condition to the transposition of their atoms is absent. Even the compounds of nitrogen, which are most liable to change, such as those which 
are found in animal bodies, do not enter into a state of putrefaction when dry.

The result of the known transformations of azotized substances proves, that the water does not merely act as a medium in which motion is permitted to the elements in the act of transposition, but that its influence depends on chemical affinity. When the decomposition of such substances is effected with the assistance of water, their nitrogen is invariably liberated in the form of ammonia. This is a fixed rule without any exceptions, whatever may be the cause which produces the decompositions. All organic compounds containing nitrogen, evolve the whole of that element in the form of ammonia when acted on by alkalies. Acids, and increase of temperature, produce the same effect. It is only when there is a deficiency of water or its elements, that cyanogen or other azotized compounds are produced.

From these facts it may be concluded, that ammonia is the most stable compound of nitrogen; and that hydrogen and nitrogen possess a degree of affinity for each other surpassing the attraction of the latter body for any other element.

Already, in considering the transformations of substances destitute of nitrogen, we have recognised the great affinity of carbon for oxygen as a powerful cause for effecting the disunion of the elements of a complex organic atom in a definite manner. But carbon is also invariably contained in azotized organic compounds, while the great affinity of nitrogen for hydrogen furnishes a new and powerful cause, facilitating the transposition of their component parts. Thus, in the bodies which do not contain nitrogen we have one element, and in those in. which that substance is present, two elements, which mutually share the elements of water. Hence there are two opposite afinities at play, which mutually strengthen each other's action.

Now we know, that the most powerful attractions may be overcome by the influence of two affinities. 
Thus, a decomposition of alumina may be effected with the greatest facility, when the affinity of charcoal for oxygen, and of chlorine for aluminium, are both put in action, although neither of these alone has any influence upon it. There is in the nature and constitution of the compounds of nitrogen a kind of tension of their component parts, and a strong disposition to yield to transformations, which effect spontaneously the transposition of their atoms on the instant that water or its elements are brought in contact with them.

The characters of the hydrated cyanic acid, one of the simplest of all the compounds of nitrogen, are perhaps the best adapted to convey a distinct idea of the manner in which the atoms are disposed of in transformations. This acid contains nitrogen, hydrogen, and oxygen, in such proportions, that the addition of a certain quantity of the elements of water is exactly sufficient to cause the oxygen contained in the water and acid to unite with the carbon and form carbonic acid, and the hydrogen of the water to combine with the nitrogen and form ammonia. The most favorable conditions for a complete transformation are, therefore, associated in these bodies, and it is well krown, that the disunion takes place on the instant in which the cyanic acid and water are brought into contact, the mixture being converted into carbonic acid and ammonia, with brisk effervescence.

This decomposition may be considered as the type of the transformations of all azotized compounds; it is putrefaction in its simplest and most perfect form, because the new products, the carbonic acid and ammonia, are incapable of further transformations.

Putrefaction assumes a totally different and much more complicated form, when the products, which are first formed, undergo a further change. In these cases the process consists of several stages, of which it is impossible to determine when one ceases and the other begins. 
The transformations of cyanogen, a body composed of carbon and nitrogen, and the simplest of all the compounds of nitrogen, will convey a clear idea of the great variety of products which are produced in such a case: it is the only example of the putrefaction of an azotized body which has been at all accurately studied.

A solution of cyanogen in water becomes turbid after a short time, and deposits a black, or brownish black matter, which is a combination of ammonia with another body, produced by the simple union of cyanogen with water. This substance is insoluble in water, and is thus enabled to resist further change.

A second transformation is effected by the cyanogen being shared between the elements of the water, in consequence of which cyanic acid is formed by a certain quantity of the cyanogen combining with the oxygen of the water, while hydrocyanic acid is also formed by another portion of the cyanogen uniting with the hydrogen which was liberated.

Cyanogen experiences a third transformation, by which a complete disunion of its elements takes place, these being divided between the constituents of the water. Oxalic acid is the one product of this disunion, and ammonia the other.

Cyanic acid, the formation of which has been mentioned above, cannot exist in contact with water, being decomposed immediately into carbonic acid and ammonia. The cyanic acid, however, newly formed in the decomposition of cyanogen, escapes this decomposition by entering into combination with the free ammonia, by which urea* is produced.

The hydrocyanic acid is also decomposed into a brown matter which contains hydrogen and cyanogen, the latter in greater proportion than it does in the gaseous state. Oxalic acid, urea, and carbonic acid, are also formed by its decomposition, and form -

* See page 87 , note. 
ic acid and ammonia are produced by the decomposition of its radical.

Thus, a substance into the composition of which only two elements (carbon and nitrogen) enter, yields eight totally different products. Several of these products are formed by the transformation of the original body, its elements being shared between the constituents of water; others are produced in consequence of a further disunion of those first formed. The urea and carbonate of ammonia are generated by the combination of two of the products, and in their formation the whole of the elements have assisted.

These examples show, that the results of decomposition by fermentation or putrefaction comprehend very different phenomena. The first kind of transformation is, the transposition of the elements of one complex compound, by which new compounds are produced with or without the assistance of the elements of water. In the products newly formed in this manner, either the same proportions of those component parts which were contained in the matter before transformation, are found, or with them, an excess, consisting of the constituents of water, which had assisted in promoting the disunion of the elements.

The second kind of transformation consists of the transpositions of the atoms of two or more complex compounds, by which the elements of both arrange themselves mutually into new products, with or without the coöperation of the elements of water. In this kind of transformation, the new products contain the sum of the constituents of all the compounds which had taken a part in the decomposition.

The first of these two modes of decomposition is that designated fermentation, the second putrefaction; and when these terms are used in the following pages, it will always be to distinguish the two processes above described, which are so different in their results. 


\section{CHAPTER V.}

FERMENTATION OF SUGAR.

The peculiar decomposition, which sugar suffers, may be viewed as a type of all the transformations designated fermentation.*

Thénard obtained from 100 grammes $\dagger$ of canesugar 0.5262 of absolute alcohol. 100 parts of sugar from the cane yield, therefore, 103.89 parts of carbonic acid and alcohol. "The entire carbon in these products is equal to 42 parts, which is exactly the quantity originally contained in the sugar.

The analysis of sugar from the cane, proves that it contains the elements of carbonic acid and alcohol, minus 1 atom of water. The alcohol and car-. bonic acid produced by the fermentation of a certain. quantity of sugar, contained together one equivalent of oxygen, and one equivalent of hydrogen, the ele-. ments, therefore, of one equivalent of water, more than the sugar contained. The excess of weight in the products is thus explained most satisfactorily; it is owing, namely, to the elements of water having taken part in the metamorphosis of the sugar.

It is known, that 1 atom of sugar contains 12 equivalents of carbon, both from the proportions in which it unites with bases, and from the composition

* When yeast is made into a thin paste with water, and 1 cubic centimetre of this mixture introduced into a graduated glass receiver filled with mercury, in which are already 19 grammes of a solution of canesugar, containing 1 gramme of pure solid sugar; it is found, after the mixture has been exposed for 24 hours to a temperature of from 20 to 25 C. $(68-77$ F.), that a volume of carbonic acid has been formed, which, at $0^{\circ} \mathrm{C}$. ( $32^{\circ} \mathrm{F}$ ) and an atmospheric pressure indicated by 0.76 metre Bar. would be from 245 to 250 cubic centimetres. But to this quantity we must add 11 cubic centimetres of carbonic acid, with which the 11 grammes of liquid would be saturated, so that in all 255 -259 cubic centimetres of carbonic acid are obtained. This volume of carbonic acid corresponds to from 0.503 to 0.5127 grammes by weight. $-L$.

$\uparrow$ The gramme equals $15 \cdot 4440$ grains. 
of saccharic acid, the product of its oxidation. Now none of these atoms of carbon are contained in the sugar as carbonic acid, because the whole quantity is obtained as oxalic acid, when sugar is treated with hypermanganate of potash (Gregory); and as oxalic acid is a lower degree of the oxidation of carbon than carbonic acid, it is impossible to conceive that the lower degree should be produced from the higher, by means of one of the most powerful agents of oxidation which we possess.

It can be also proved, that the hydrogen of the sugar does not exist in it in the form of alcohol, for it is converted into water and a kind of carbonaceous matter, when treated with acids, particularly with such as contain no oxygen; and this manner of decomposition is never suffered by a compound of alcohol.

Sugar contains, therefore, neither alcohol nor carbonic acid, so that these bodies must be produced by a different arrangement of its atoms, and by their union with the elements of water.

In this metamorphosis of sugar, the elements of the yeast, by contact with which its fermentation was effected, take no appreciable part in the transposition of the elements of the sugar; for in the products resulting from the action, we find no component part of this substance.

We may now study the fermentation of a vegetable juice, which contains not only saccharine matter, but also such substances as albumen and gluten. The juices of parsnips, beet-roots, and onions, are well adapted for this purpose. When such a juice is mixed with yeast at common temperatures, it ferments like a solution of sugar. Carbonic acid gas escapes from it with effervescence, and in the liquid, alcohol is found in quantity exactly corresponding to that of the sugar originally contained in the juice. But such a juice undergoes spontaneous decomposition at a temperature of from $95^{\circ}$ to $104^{\circ}\left(35^{\circ}-40^{\circ}\right.$ C.). Gases possessing an offensive smell are evolved 
in considerable quantity, and when the liquor is examined after the decomposition is completed, no alcohol can be detected. The sugar has also disappeared, and with it all the azotized compounds which existed in the juice previously to its fermentation. Both were decomposed at the same time; the nitrogen of the azotized compounds remains in the liquid as ammonia, and, in addition to it, there are three new products, formed from the component parts of the juice. One of these is lactic acid, the slightly volatile compound found in the animal organism; the other is the crystalline body, which forms the principal constituent of manna; and the third is a mass resembling gum-arabic, which forms a thick viscous solution with water. These three products weigh more than the sugar contained in the juice, even without calculating the weight of the gaseous products. Hence; they are not produced from the elements of the sugar alone. None of these three substances could be detected in the juice before fermentation. They must, therefore, have been formed by the interchange of the elements of the sugar with those of the foreign substances also present. It is this mixed transformation of two or more compounds which receives the special name of putrefaction.

YEAST OR FERMENT.

When attention is directed to the condition of those substances, which possess the power of inducing fermentation and putrefaction in other bodies, evidences are found in their general characters, and in the manner in which they combine, that they all are bodies, the atoms of which are in the act of transposition.

The characters of the remarkable matter, which is deposited in an insoluble state during the fermentation of beer, wine, and vegetable juices, may first be studied. 
This substance, which has been called yeast or ferment, from the power which it possesses of causing fermentation in sugar, or saccharine vegetable juices, possesses all the characters of a compound of nitrogen in the state of putrefaction and eremacausis.

Like wood in the state of eremacausis, yeast converts the oxygen of the surrounding air into carbonic acid, but it also evolves this gas from its own mass, like bodies in the state of putrefaction. (Colin.) When kept under water, it emits carbonic acid, accompanied by gases of an offensive smell, (Thénard,) and is at last converted into a substance resembling old cheese. (Proust.) But when its own putrefaction is completed, it has no longer the power of inducing fermentation in other bodies. The presence of water is quite necessary for sustaining the properties of ferment, for by simple pressure its power to excite fermentation is much diminished, and is completely destroyed by drying. Its action is arrested also by the temperature of boiling water, by alcohol, common salt, an excess of sugar, oxide of mercury, corrosive sublimate, pyroligneous acid, sulphurous acid, nitrate of silver, volatile oils, and in short by all antiseptic substances.

The insoluble part of the substance called ferment does not cause fermentation. For when the yeast from wine or beer is carefully washed with water, care being taken that it is always covered with this fluid, the residue does not produce fermentation.

The soluble part of ferment likewise does not excite fermentation. An aqueous infusion of yeast may be mixed with a solution of sugar, and preserved in vessels from which the air is excluded, without either experiencing the slightest change. What then, we may ask, is the matter in ferment which excites fermentation, if neither the soluble nor insoluble parts possess the power? This question has been answered by Colin in the most satisfactory manner. $\mathrm{He}$ has shown, that in reality it is the soluble part. But before it obtains this power, the decanted infu- 
sion must be allowed to cool in contact with the air, and to remain some time exposed to its action. When introduced into a solution of sugar in this state, it produces a brisk fermentation; but without previous exposure to the air, it manifests no such property.

The infusion absorbs oxygen during its exposure to the air, and carbonic acid may be found in it after a short time.

Yeast produces fermentation in consequence of the progressive decomposition, which it suffers from the action of air and water.

Now when yeast is made to act on sugar, it is found, that after the transformation of the latter substance into carbonic acid and alcohol is completed, part of the yeast itself has disappeared.

From 20 parts of fresh yeast from beer, and 100 parts of sugar, Thénard obtained, after the fermentation was completed, 13.7 parts of an insoluble residue, which diminished to 10 parts when employed in the same way with a fresh portion of sugar. These ten parts were white, possessed of the properties of woody fibre, and had no further action on sugar.

It is evident, therefore, that during the fermentation of sugar by yeast, both of these substances suffer decomposition at the same time, and disappear in consequence. But if yeast be a body which excites fermentation by being itself in a state of decomposition, all other matters in the same condition should have a similar action upon sugar; and this is in reality the case. Muscle, urine, isinglass, osmazome, ${ }^{*}$ albumen, cheese, gliadine, gluten, legumin, and blood, when in a state of putrefaction, have all the power of producing the putrefaction, or fermentation of a solution of sugar. Yeast, which by continued washing has entirely lost the property of inducing fermentation, regains it when its putrefaction

* An extractive animal matter on which the peculiar flavor of broth is supposed to depend; hence its name, from the Greek for odor and broth. 
has recommenced, in consequence of its being kept in a warm situation for some time.

Yeast and putrefying animal and vegetable matters act as peroxide of hydrogen does on oxide of silver, when they induce bodies with which they are in contact to enter into the same state of decomposition. The disturbance in the attraction of the constituents of the peroxide of hydrogen effects a disturbance in the attraction of the elements of the oxide of silver, the one being decomposed, on account of the decomposition of the other.

Now if we consider the process of the fermentation of pure sugar, in a practical point of view, we meet with two facts of constant occurrence. When the quantity of ferment is too small in proportion to that of the sugar, its putrefaction will be completed before the transformation of all the sugar is effected. Some sugar here remains undecomposed, because the cause of its transformation is absent, viz. contact with a body in a state of decomposition.

But when the quantity of ferment predominates, a certain quantity of it remains after all the sugar has fermented, its decomposition proceeding very slowly, on account of its insolubility in water. This residue of ferment is still able to induce fermentation, when introduced into a fresh solution of sugar, and retains the same power until it has passed through all the stages of its own transformation. Hence, a certain quantity of yeast is necessary in order to effect the transformation of a certain portion of sugar, not because it acts by its quantity in increasing any affinity, but because its influence depends solely on its presence, and its presence is necessary, until the last atom of sugar is decomposed.

These facts and observations point out the existence of a new cause, which effects combinations and decompositions. This cause is the action which bodies in a state of combination or decomposition exercise upon substances, the component parts of which are united together by a feeble affinity. This 
action resembles a peculiar power, attached to a body in the state of combination or decomposition, but exerting its influence beyond the sphere of its own attractions. We are now able to account satisfactorily for many known phenomena.

A large quantity of hippuric acid may be obtained from the fresh urine of a horse, by the addition of muriatic acid; but when the urine has undergone putrefaction, no trace of it can be discovered. The urine of man contains a considerable quantity of urea; but when the urine putrefies, the urea entirely disappears. When urea is added to a solution of sugar in the state of fermentation, it is decomposed into carbonic acid and ammonia. No asparagin* can be detected in a putrefied infusion of asparagin, liquorice-root, or the root of marshmallow (Althea officinalis).

It has already been mentioned, that the strong affinity of nitrogen for hydrogen, and that of carbon for oxygen, are the cause of the facility with which the elements of azotized compounds are disunited; those affinities aiding each other, inasmuch as by virtue of them different elements of the compounds strive to take possession of the different elements of water. Now since it is found that no body destitute of nitrogen, possesses, when pure, the property of decomposing spontaneously whilst in contact with water, we must ascribe this property which azotized bodies possess in so eminent a degree, to something peculiar in the nature of the compounds of nitrogen, and to their constituting, in a certain measure, more highly organized atoms.

Every azotized constituent of the animal or vegetable organism runs spontaneously into putrefaction, when exposed to moisture and a high temperature.

Azotized matters are, accordingly, the only causes of fermentation and putrefaction in vegetable substances.

* A peculiar principle obtained from asparagus. See Brande's Chemistry, p. 1042. 
Putrefaction, on account of its effects, as a mixed transformation of many different substances, may be classed with the most powerful processes of deoxidation, by which the strongest affinities are overcome.

When a solution of gypsum in water is mixed with a decoction of sawdust, or any other organic matter capable of putrefaction, and preserved in well-closed vessels, it is found after some time, that the solution contains no more sulphuric acid, but in its place carbonic and free hydrosulphuric acid, between which the lime of the gypsum is shared. In stagnant water containing sulphates in solution, crystallized pyrites is observed to form on the decaying roots.

Now we know, that in the putrefaction of wood under water, when air therefore is excluded, a part of its carbon combines with the oxygen of the water, as well as with the oxygen which the wood itself contains; whilst its hydrogen and that of the decomposed water are liberated either in a pure state, or as carburetted hydrogen. The products of this decomposition are of the same kind as those generated when steam is conducted over red-hot charcoal.

It is evident, that if with the water a substance containing a large quantity of oxygen, such as sulphuric acid, be also present, the matters in the state of putrefaction will make use of the oxygen of that substance as well as that of the water, in order to form carbonic acid; and the sulphur and hydrogen being set free will combine whilst in the nascent state, producing hydrosulphuric acid, which will be again decomposed if metallic oxides be present; and the results of this second decomposition will be water and metallic sulphurets.

'The putrefied leaves of woad (Isatis tinctoria), in contact with indigo-blue, water, and alkalies, suffer further decomposition, and the indigo is deoxidized and dissolved.

The mannite formed by the putrefaction of beetroots and other plants which contain sugar, contains 
the same number of equivalents of carbon and hydrogen as the sugar of grapes, but two atoms less of oxygen; and it is highly probable that it is produced from sugar of grapes, contained in those plants, in precisely the same manner as indigo-blue is converted into deoxidized white indigo.

During the putrefaction of gluten, carbonic acid and pure hydrogen gas are evolved; phosphate, acetate, caseate, and lactate of ammonia being at the same time produced in such quantity, that the further decomposition of the gluten ceases. But when the supply of water is renewed, the decomposition begins again, and in addition to the salts just mentioned, carbonate of ammonia and a white crystalline matter resembling mica (caseous oxide) are formed, together with hydrosulphate of ammonia, and a mucilaginous substance coagulable by chlorine. Lactic acid is almost always produced by the putrefaction of organic bodies.

We may now compare fermentation and putrefaction with the decomposition which organic compounds suffer under the influence of a high temperature. Dry distillation would appear to be a process of combustion or oxidation going on in the interior of a substance, in which a part of the carbon unites with all or part of the oxygen of the compound, while other new compounds containing a large proportion of hydrogen are necessarily produced. Fermentation may be considered as a process of combustion or oxidation of a similar kind, taking place in a liquid between the elements of the same matter, at a very slightly elevated temperature; and putrefaction as a process of oxidation, in which the oxygen of all the substances present comes into play. 


\section{CHAPTER VI.}

\section{EREMACAUSIS, OR DECAY.}

IN organic nature, besides the processes of decomposition named fermentation and putrefaction, another and not less striking class of changes occurs, which bodies suffer from the influence of the air. This is the act of gradual combination of the combustible elements of a body with the oxygen of the air; a slow combustion or oxidation, to-which we shall apply the term of eremacausis.

The conversion of wood into humus, the formation of acetic acid out of alcohol, nitrification, and numerous other processes, are of this nature. Vegetable juices of every kind, parts of animal and vegetable substances, moist sawdust, blood, \&c., cannot be exposed to the air, without suffering immediately a progressive change of color and properties, during which oxygen is absorbed. These changes do not take place when water is excluded, or when the substances are exposed to the temperature of $32^{\circ}$, and it has been observed that different bodies require different degrees of heat, in order to effect the absorption of oxygen, and, consequently, their eremacausis. 'The property of suffering this change is possessed in the highest degree by substances containing nitrogen.

When vegetable juices are evaporated by a gentle heat in the air, a brown or brownish-black substance is precipitated as a product of the action of oxygen upon them. This substance, which appears to possess similar properties from whatever juice it is obtained, has received the name of extractive matter; it is insoluble or very sparingly soluble in water, but is dissolved with facility by alkalies. By the action of air on solid animal or vegetable matters, a similar 
oulverulent brown substance is formed, and is known oy the name of humus.

The conditions which determine the commencement of eremacausis are of various kinds. Many organic substances, particularly such as are mixtures of several more simple matters, oxidize in the air when simply moistened with water; others not until they are subjected to the action of alkalies; but the greatest part of them undergo this state of slow combustion or oxidation, when brought in contact with other decaying matters.

The eremacausis of an organic matter is retarded or completely arrested by all those substances which prevent fermentation or putrefaction. Mineral acids, salts of mercury, aromatic substances, empyreumatic oils, and oil of turpentine, possess a similar action in this respect. The latter substances have the same effect on decaying bodies as on phosphuretted hydrogen, the spontaneous inflammability of which they destroy.

Many bodies which do not decay when moistened with water, enter into eremacausis when in contact with an alkali. Gallic acid, hæmatin,* and many other compounds, may be dissolved in water and yet remain unaltered; but if the smallest quantity of a free alkali is present, they acquire the property of attracting oxygen, and are converted into a brown substance like humus, evolving very frequently at the same time carbonic acid. (Chevreul.)

A very remarkable kind of eremacausis takes place in many vegetable substances, when they are exposed to the influence of air, water, and ammonia. They absorb oxygen very rapidly, and form splendid violet or red-colored liquids, as in the case of orcin and erythrin. They now contain an azotized substance, not in the form of ammonia.

All these facts show, that the action of oxygen seldom affects the carbon of decaying substances, 
and this corresponds exactly to what happens in combustion at high temperatures. It is well known, for example, that when no more oxygen is admitted. to a compound of carbon and hydrogen than is sufficient to combine with its hydrogen, the carbon is not burned, but is separated as lampblack; * while, if the quantity of oxygen is not sufficient even to consume all the hydrogen, new compounds are formed, such as napthalin $†$ and similar matters, which contain a smaller proportion-of hydrogen than those compounds of carbon and hydrogen which previously existed in the combustible substance.

There is no example of carbon combining directly with oxygen at common temperatures, but numerous facts show that hydrogen, in certain states of condensation, possesses that property. Lampblack which has been heated to redness may be kept in contact with oxygen gas, without forming carbonic acid; but lampblack, impregnated with oils which contain a large proportion of hydrogen, gradually becomes warm, and inflames spontaneously. The spontaneous inflammability of the charcoal used in the fabrication of gunpowder has been correctly ascribed to the hydrogen, which it contains in considerable quantity; for during its reduction to powder, no trace of carbonic acid can be detected in the air surrounding it; it is not formed until the temperature of the mass has reached a red heat. The heat which produces the inflammation is, therefore, not caused by the oxidation of the carbon.

The substances which undergo eremacausis may be divided into two classes. 'The first class comprehends those substances which unite with the oxygen of the air, without evolving carbonic acid; and the second, such as emit carbonic acid by absorbing oxygen.

When the oil of bitter almonds is exposed to the

* As in the combustion of spirits of turpentine, now much employed, under various names, in lamps.

$\dagger$ A substance obtained from coal tar. 
air, it absorbs two equivalents of oxygen, and is converted into benzoic acid; but half of the oxygen absorbed combines with the hydrogen of the oil, and forms water, which remains in union with the anhydrous benzoic acid.*

But, although it appears very probable that the oxygen acts primarily and principally upon hydrogen, the most combustible constituent of organic matter in the state of decay; still it cannot thence be concluded, that the carbon is quite devoid of the power to unite with oxygen, when every particle of it is surrounded with hydrogen, an element with which the oxygen combines with greater facility.

We know, on the contrary, that although nitrogen cannot be made to combine with oxygen directly, yet it is oxidized and forms nitric acid, when mixed with a large quantity of hydrogen, and burned in oxygen gas. In this case its affinity is evidently increased by the combustion of the hydrogen, which is in fact communicated to it. It is conceivable, that in a similar manner, the carbon may be directly oxidized in several cases, obtaining from its contact with hydrogen in eremacausis a property which it does not itself possess at common temperatures. But the formation of carbonic acid during the eremacausis of bodies containing hydrogen, must in most cases be ascribed to another cause. It appears.

* According to the experiments of Döbereiner, 100 parts of pyrogallic acid absorb 38.09 parts of oxygen when in contact with ammonia and water; the acid being changed in consequence of this absorption into a mouldy substance, which contains less oxygen than the acid itself. It is evident, that the substance which is formed is not a higher oxide; and it is found, on comparing the quantity of the oxygen absorbed with that of the hydrogen contained in the acid, that they are exactly in the proportions for forming water.

When colorless orcin is exposed together with ammonia to the contact of oxygen gas, the beautiful red-colored orcein is produced. Now.. the only changes which take place here are, that the absorption of oxygen by the elements of orcin and ammonia causes the formation of. water ; 1 equivalent of orcin $\mathrm{C} 18 \mathrm{H} 12 \mathrm{O}$, and 1 equivalent of ammonia NH3, absorb 5 equivalents of oxygen, and 5 equivalents of water are produced, the composition of orcein being $\mathrm{C} 18 \mathrm{H} 10 \mathrm{O} 8 \mathrm{~N}$. (Dumas.) In this case it is evident, that the oxygen absorbed has united: merely with the hydrogen. $-L$. 
to be formed in a manner similar to the formation of acetic acid, by the eremacausis of saliculite of potash.*

An alkaline solution of hæmatin being exposed to an atmosphere of oxygen, $0.2 \mathrm{grm}$. absorb $28 \cdot 6$ cubic centimeters of oxygen gas in twenty-four hours, the alkali acquiring at the same time 6 cubic centimeters of carbonic acid. (Chevreul.) But these 6 cubic centimeters of carbonic acid contain only an equal rolume of oxygen, so that it is certain from this experiment, that $\frac{3}{4}$ of the oxygen absorbed have not united with the carbon. It is highly probable, that during the oxidation of the hydrogen, a portion of the carbon had united with the oxygen contained in the hæmatin, and had separated from the other elements as carbonic acid.

The experiments of De Saussure upon the decay of woody fibre show, that such a separation is quite possible. Moist woody fibre evolved one volume of carbonic acid for every volume of oxygen which it absorbed. It has just been mentioned, that carbonic acid contains its own volume of oxygen. Now, woody fibre contains carbon and the elements of water, so that the result of the action of oxygen upon it is exactly the same as if pure charcoal had combined directly with oxygen. But the characters of woody fibre show, that the elements of water are not contained in it in the form of. water ; for, were this the case, starch, sugar, and gum must also be considered as hydrates of carbon.

But if the hydrogen does not exist in woody fibre in the form of water, the direct oxidation of the carbon cannot be considered as at all probable, without rejecting all the facts established by experiment regarding the process of combustion at low temperatures.

* This salt, when exposed to a moist atmosphere, absorbs 3 atoms of oxygen; melanic acid is produced, a body resembling humus, in consequence of the formation of which, the elements of 1 atom of acetic acid are separated from the saliculous acid. $-L$. 
If we examine the action of oxygen upon a substance containing a large quantity of hydrogen, such as alcohol, we find most distinctly, that the direct formation of carbonic acid is the last stage of its oxidation, and that it is preceded by a series of changes, the last of which is a complete combustion of the hydrogen. Aldehyde, acetic, formic, oxalic, and carbonic acids, form a connected chain of products arising from the oxidation of alcohol; and the. successive changes which this fluid experiences from the action of oxygen may be readily traced in them. Aldehyde is alcohol minus hydrogen; acetic acid is formed by the direct union of aldehyde with oxygen. Formic acid and water are formed by the union of acetic acid with oxygen. When all the hydrogen is removed from this formic acid, oxalic acid is produced; and the latter acid is converted into carbonic acid by uniting with an additional portion of oxygen. All these products appear to be formed simultaneously, by the action of oxidizing agents on alcohol; but it can scarcely be doubted, that the formation of the last product, the carbonic acid, does not take place until all the hydrogen has been abstracted.

The absorption of oxygen by drying oils certainly does not depend upon the oxidation of their carbon; for in raw nut-oil, for example, which was not free from mucilage and other substances, only twenty-one volumes of carbonic acid were formed for every 146 volumes of oxygen gas absorbed.

It must be remembered, that combustion or oxidation at low temperatures produces results quite similar to combustion at high temperatures with limited access of air. The most combustible element of a compound, which is exposed to the action of oxygen, must become oxidized first, for its superior combustibility is caused by its being enabled to unite with oxygen at a temperature at which the other elements cannot enter into that combination; this property having the same effect as a greater affinity. 
The combustibility of potassium is no measure of its affinity for oxygen; we have reason to believe that the attraction of magnesium and aluminium for oxygen is greater than that of potassium for the same element; but neither of those metals oxidizes either in air or water at common temperatures, whilst potassium decomposes water with great violence, and appropriates its oxygen.

Phosphorus and hydrogen combine with oxygen at ordinary temperatures, the first in moist air, the second when in contact with finely-divided platinum; while charcoal requires a red heat before it can enter into combination with oxygen. It is evident, that phosphorus and hydrogen are more combustible than charcoal, that is, that their affinity for oxygen at common temperatures is greater; and this is not the less certain, because it is found, that carbon in certain other conditions shows a much greater affinity for oxygen than either of those substances.

In putrefaction, the conditions are evidently present, under which the affinity of carbon for oxygen comes into play; neither expansion, cohesion, nor the gaseous state, opposes it, whilst in eremacausis all these restraints have to be overcome.

The evolution of carbonic acid, during the decay or eremacausis of animal or vegetable bodies which are rich in hydrogen, must accordingly be ascribed to a transposition of the elements or disturbance in their attractions, similar to that which gives rise to the formation of carbonic acid in the processes of fermentation and putrefaction.

The eremacausis of such substances is, therefore, a decomposition analogous to the putrefaction of azotized bodies. For in these there are two affinities at play; the affinity of nitrogen for hydrogen, and that of carbon for oxygen, and both facilitate the disunion of the elements. Now there are two affinities also in action in those bodies which decay with the evolution of carbonic acid. One of these affinities is the attraction of the oxygen of the air for the 
hydrogen of the substance, which corresponds to the attraction of nitrogen for the same element; and the other is the affinity of the carbon of the substance for its oxygen, which is constant under all circumstances.

When wood putrefies in marshes, carbon and oxygen are separated from its elements in the form of carbonic acid, and hydrogen in the form of carburetted hydrogen. But when wood decays or putrefies in the air, its hydrogen does not combine with carbon, but with oxygen, for which it has a much greater affinity at common temperatures.

Now it is evident, from the complete similarity of these processes, that decaying and putrefying bodies can mutually replace one another in their reciprocal actions.

All putrefying bodies pass into the state of decay, when exposed freely to the air, and all decaying matters into that of putrefaction when air is excluded. All bodies, likewise, in a state of decay are capable of inducing putrefaction in other bodies in the same manner as putrefying bodies themselves do.

\section{CHAPTER VII.}

\section{EREMACAUSIS OR DECAY OF BODIES DESTITUTE OF} NITROGEN: FORMATION OF ACETIC ACID.

All those substances which appear to possess the property of entering spontaneously into fermentation and putrefaction, do not in reality suffer those changes without some previous disturbance in the attraction of their elements. Eremacausis always precedes fermentation and putrefaction, and it is not until after the absorption of a certain quantity of oxygen that the signs of a transformation in the interior of the substances show themselves. 
It is a very general error to suppose that organic substances have the power of undergoing change spontaneously, without the aid of an external cause. When they are not in a state of change, it is necessary, before they can assume that state, that the existing equilibrium of their elements should be disturbed; and the most common cause of this disturbance is undoubtedly the atmosphere which surrounds all bodies.

The juices of the fruit or other part of a plant which very readily undergo decomposition, retain their properties unchanged as long as they are protected from immediate contact with the air, that is, as long as the cells or organs in which they are contained resist the influence of the air. It is not until after the juices have, been exposed to the air, and have absorbed a certain quantity of oxygen, that the substances dissolved in them begin to be decomposed.

The beautiful experiments of Gay-Lussac upon the fermentation of the juice of grapes, as well as the important practical improvements to which they. have led, are the best proofs that the atmosphere possesses an influence upon the changes of organic substances. The juice of grapes which were expressed under a receiver filled with mercury, so that air was completely excluded, did not ferment. But when the smallest portion of air was introduced, a certain quantity of oxygen became absorbed, and fermentation immediately began. Although the juice was expressed from the grapes in contact with air, under the conditions therefore necessary to cause its fermentation, still this change did not ensue when the juice was heated in close vessels to the temperature of boiling water. When thus treated, it could be preserved for years without losing its property of fermenting. A fresh exposure to the air at any period caused it to ferment.

Animal food of every kind, and even the most delicate vegetables, may be preserved unchanged if 
heated to the temperature of boiling water in ressels from which the air is completely excluded. Food thus prepared has been kept for fifteen years, and upon opening the vessels, after this long time, has been found as fresh and well-flavoured as when originally placed in them.*

The action of the oxygen in these processes of decomposition is very simple; it excites changes in the composition of the azotized matters dissolved in the juices, - the mode of combination of the elements of those matters undergoes a disturbance and change in consequence of their contact with oxygen. The oxygen acts here in a similar manner to the friction or motion which affects the mutual decomposition of two salts, the crystallization of salts from their solution, or the explosion of fulminating mercury. It causes the state of rest to be converted into a state of motion.

When this condition of intestine motion is once excited, the presence of oxygen is no longer necessary. The smallest particle of an azotized body in this act of decomposition exercises an influence upon the particles in contact with it, and the state of motion is thus propagated through the substance. The air may now be completely excluded, but the

* The process is as follows: Let the substance to be preserved be first parboiled, or rather somewhat more, the bones of the meat being previously removed. Put the meat into a tin cylinder, fill up the vessel with seasoned rich soup, and then solder on the lid, pierced with a small hole. When this has been done, let the tin vessel thus prepared be placed in brine and heated to the boiling point, to complete the cooking of the meat. The hole of the lid is now to be closed by soldering, whilst the air is rarefied. The vessel is then allowed to cool, and from the diminution of volume, in consequence of the reduction of temperature, both ends of the cylinder are pressed inwards and become concave. The tin cases, thus hermetically sealed, are exposed in a test-chamber, for at least a month, to a temperature above what they are ever likely to encounter; from $90^{\circ}$ to $110^{\circ} \mathrm{F}$. If the process has failed, putrefaction takes place, and gas is evolved, which will cause the ends of the case to bulge, so as to render them convex, instead of concave. But the contents of those cases which stand the test will infallibly keep perfectly sweet and good in any climate, and for any number of years. If there be any taint about the meat when put up, it inevitably ferments, and is detected in the proving process. - URE's Dict. of Arts and Manuf. 
fermentation or putrefaction proceeds uninterruptedly to its completion. It has been remarked, that the mere contact of carbonic acid is sufficient to produce fermentation in the juices of several fruits.

The contact of ammonia and alkalies in general may be mentioned amongst the chemical conditions, which determine the commencement of eremacausis; for their presence causes many substances to absorb oxygen and to decay, in which neither oxygen nor alkalies alone produce that change.

Thus alcohol does not combine with the oxygen of the air at common temperatures. But a solution of potash in alcohol absorbs oxygen with much rapidity, and acquires a brown color. The alcohol is found after a short time to contain acetic acid, formic acid, and the products of the decomposition of aldehyde by alkalies, including aldehyde resin, which gives the liquid a brown color.

The most general condition for the production of eremacausis in organic matter is contact with a body already in the state of eremacausis or putrefaction. We have here an instance of true contagion; for the communication of the state of combustion is in reality the effect of the contact.

It is decaying wood which causes fresh wood around it to assume the same condition, and it is the very finely divided woody fibre in the act of decay which in moistened gall-nuts converts the tannic acid with such rapidity into gallic acid.

A most remarkable and decided example of this induction of combustion has been observed by $\mathrm{De}$ Saussure. It has already been mentioned, that moist woody fibre, cotton, silk, or vegetable mould, in the act of fermentation or putrefaction, converts oxygen gas which may surround it into carbonic acid, without change of volume. : Now, De Saussure added a certain quantity of hydrogen gas to the oxygen, and observed a diminution in volume immediately after the addition. A part of the hydrogen gas had disappeared, and along with it a portion of the oxy- 
gen, but a corresponding quantity of carbonic acid gas had not been formed. The hydrogen and oxygen had disappeared in exactly the same proportion as that in which they combine to form water; a true combustion of the hydrogen, therefore, had been induced by mere contact with matter in the state of eremacausis. The action of the decaying. substance here produced results exactly similar to those effected by spongy platinum; but that they proceeded from a different cause was shown by the fact that the presence of carbonic oxide, which arrests completely the action of platinum on carburetted hydrogen, did not retard in the slightest degree the combustion of the hydrogen in contact with the decaying bodies.

But the same bodies were found by De Saussure not to possess the property just described, before they were in a state of fermentation or decay; and he has shown that even when they are in this state, the presence of antiseptic matter destroys completely all their influence.

Let us suppose a volatile substance containing a large quantity of hydrogen to be substituted for the hydrogen gas in De Saussure's experiments. Now, the hydrogen in such compounds being contained in a state of greater condensation would suffer a more rapid oxidation, that is, its combustion would be soorier completed. This principle is in reality attended to in the manufactories in which acetic acid is prepared according to the new plan. In the process there adopted all the conditions are afforded for the eremacausis of alcohol, and for its consequent conversion into acetic acid.

The alcohol is exposed to a moderate heat, and spread over a very extended surface, but these conditions are not sufficient to effect its oxidation. The alcohol must be mixed with a substance which is with facility changed by the oxygen of the air, and either enters into eremacausis by mere contact with oxygen, or by its fermentation or putrefaction 
yields products possessed of this property. A small quantity of beer, acescent wine, a decoction of malt, honey, and numerous other substances of this kind, possess the action desired.

The difference in the nature of the substances which possess this property shows, that none of them can contain a peculiar matter which has the property of exciting eremacausis; they are only the bearers of an action, the influence of which extends beyond the sphere of its own attractions. Their power consists in a condition of decomposition or eremacausis, which impresses the same condition upon the atoms of alcohol in its vicinity; exactly as in the case of an alloy of platinum and silver dissolving in nitric acid, in which the platinum becomes oxidized, by virtue of an inductive action exercised upon it by the silver in the act of its oxidation. The hydrogen of the alcohol is oxidized at the expense of the oxygen in contact with it, and forms water, evolving heat at the same time; the residue is aldehyde, a substance which has as great an affinity for oxygen as sulphurous acid, and combines, therefore, directly with it, producing acetic acid.

\section{CHAP'TER VIII.}

EREMACAUSIS OF SUBSTANCES CONTAINING NITROGEN. NITRIFICATION.

When azotized substances are burned at high temperatures, their nitrogen does not enter into direct combination with oxygen. The knowledge of this fact is of assistance in considering the process of the eremacausis of such substances. Azotized organic matter always contains carbon and hydrogen, both of which elements have a very strong affinity for oxygen.

Now nitrogen possesses a very feeble affinity for 
that element, so that its compounds during their combustion present analogous phenomena to those which are observed in the combustion of substances containing a large proportion of hydrogen and carbon; a separation of the carbon of the latter substances in an uncombined state takes place, and in the same way the substances containing nitrogen give out that element in its gaseous form.

When a moist azotized animal matter is exposed to the action of the air, ammonia is always liberated; nitric acid is never formed.

But when alkalies or alkaline bases are present, a union of oxygen with the nitrogen takes place under the same circumstances, and nitrates are formed together with the other products of oxidation.

Although we see the most simple means and direct methods employed in the great processes of decomposition which proceed in nature, still we find that the final result depends on a succession of actions, which are essentially influenced by the chemical nature of the bodies submitted to decomposition.

When it is observed that the character of a substance remains unaltered in a whole series of phenomena, there is no reason to ascribe a new character to it, for the purpose of explaining a single phenomenon, especially where the explanation of that according to known facts offers no difficulty.

The most distinguished philosophers suppose that the nitrogen in an animal substance, when exposed to the action of air, water, and alkaline bases, obtains the power to unite directly with oxygen, and form nitric acid, but we are not acquainted with a single fact which justifies this opinion. It is only by the interposition of a large quantity of hydrogen in the state of combustion or oxidation, that nitrogen can be converted into an oxide.

When a compound of nitrogen and carbon, such as cyanogen, is burned in oxygen gas, its carbon alone is oxidized; and when it is conducted over a metallic oxide heated to redness, an oxide of nitro- 
gen is very rarely produced, and never when the carbon is in excess. Kuhlmann found in his experiments, that it was only when cyanogen was mixed with an excess of oxygen gas, and conducted over spongy platinum, that nitric acid was generated.

Kuhlmann could not succeed in causing pure nitrogen to combine directly with oxygen, even under the most favorable circumstances; thus, with the aid of spongy platinum at different temperatures, no union took place.

The carbon in the cyanogen gas must, therefore, have given rise to the combustion of the nitrogen by induction.

On the other hand we find that ammonia (a compound of hydrogen and nitrogen) cannot be exposed to the action of oxygen, without the formation of an oxide of nitrogen, and in consequence the production of nitric acid.

It is owing to the great facility with which ammonia is converted into nitric acid, that it is so difficult to obtain a correct determination of the quantity of nitrogen in a compound subjected to analysis, in which it is either contained in the form of ammonia, or from which ammonia is formed by an elevation of temperature. For when ammonia is passed over red-hot oxide of copper, it is converted, either completely or partially, into binoxide of nitrogen.

When ammoniacal gas is conducted over peroxide of manganese or iron heated to redness, a large quantity of nitrate of ammonia is obtained, if the ammonia be in excess; and the same decomposition happens when ammonia and oxygen are together passed over red-hot spongy platinum.

It appears, therefore, that the combination of oxygen with nitrogen occurs rarely during the combustion of compounds of the latter element with carbon, but that nitric acid is always a product when ammonia is present in the substance exposed to oxidation.

The cause wherefore the nitrogen in ammonia 
exhibits such a strong disposition to become converted into nitric acid is, undoubtedly, that the two products, which are the result of the oxidation of the constituents of ammonia, possess the power of uniting with one another. Now this is not the case in the combustion of compounds of carbon and nitrogen; here one of the products is carbonic acid, which, on account of its gaseous form, must oppose the combination of the oxygen and nitrogen, by preventing their mutual contact, while the superior affinity of its carbon for the oxygen during the act of its formation will aid this effect.

When sufficient access of air is admitted during the combustion of ammonia, water is formed as well as nitric acid, and both of these bodies combine together. The presence of water may, indeed, be considered as one of the conditions essential to nitrification, since nitric acid cannot exist without it.

Eremacausis is a kind of putrefaction, differing from the common process of putrefaction, only in the part which the oxygen of the air plays in the transformations of the body in decay. When this is remembered, and when it is considered that in the transposition of the elements of azotized bodies their nitrogen assumes the form of ammonia, and that in this form, nitrogen possesses a much greater disposition to unite with oxygen than it has in any of its other compounds; we can with difficulty resist the conclusion, that ammonia is the general cause of nitrification on the surface of the earth.

Azotized animal matter is not, therefore, the immediate cause of nitrification; it contributes to the production of nitric acid only in so far as it is a slow and continued source of ammonia.

Now it has been shown in the former part of this work, that ammonia is always present in the atmosphere, so that nitrates might thence be formed in substances which themselves contained no azotized matter. It is known, also, that porous substances possess generally the power of condensing ammonia; 
there are few ferruginous earths which do not evolve ammoniacal products when heated to redness, and ammonia is the cause of the peculiar smell perceived upon moistening aluminous minerals. Thus, ammonia, by being a constituent of the atmosphere, is a very widely diffused cause of nitrification, which will come into play whenever the different conditions necessary for the oxidation of ammonia are combined. It is probable, that other organic bodies in the state of eremacausis are the means of causing the combustion of ammonia; at all events, the cases are very rare, in which nitric acid is generated from ammonia, in the absence of all matter capable of eremacausis.

From the preceding observations on the causes of fermentation, putrefaction, and decay, we may now draw several conclusions calculated to correct the views generally entertained respecting the fermentation of wine and beer, and several other important processes of decomposition which occur in nature.

\section{CHAPTER IX.}

ON VINOUS FERMENTATION :-WINE AND BEER.

Ir has already been mentioned, that fermentation is excited in the juice of grapes by the access of air; alcohol and carbonic acid being formed by the decomposition of the sugar contained in the fluid. But it was also stated, that the process once commenced, continues until all the sugar is completely decomposed, quite independently of any further influence of the air.

In addition to the alcohol and carbonic acid formed by the fermentation of the juice, there is also produced a yellow or gray insoluble substance, containing a large quantity of nitrogen. It is this body which possesses the power of inducing fermentation 
in a new solution of sugar, and which has in consequence received the name of ferment.

The alcohol and carbonic acid are produced from the elements of the sugar, and the ferment from those azotized constituents of the grape-juice, which have been termed gluten, or vegetable albumen.

According to the experiments of De Saussure, fresh impure gluten evolved, in five weeks, twentyeight times its volume of a gas which consisted $\frac{3}{4}$ of carbonic acid, and $\frac{1}{4}$ of pure hydrogen gas; ammoniacal salts of several organic acids were formed at the same time. Water must, therefore, be decomposed during the putrefaction of gluten; the oxygen of this water must enter into combination with some of its constituents, whilst hydrogen is liberated, a circumstance which happens only in decompositions of the most energetic kind. Neither ferment nor any substance similar to it is formed in this case; and we have seen that in the fermentation of saccharine vegetable juices, no escape of hydrogen gas takes place.

It is evident, that the decomposition which gluten suffers in an isolated state, and that which it undergoes when dissolved in a vegetable juice, belong to two different kinds of transformations. There is reason to believe, that its change to the insoluble state depends upon an absorption of oxygen, for its separation in this state may be effected, under certain conditions, by free exposure to the air, without the presence of fermenting sugar. It is known also that the juice of grapes, or vegetable juices in general, become turbid when in contact with air, before fermentation commences; and this turbidness is owing to the formation of an insoluble precipitate of the same nature as ferment.

From the phenomena which have been observed during the fermentation of wort, ${ }^{*}$ it is known with

* Wort is an infusion of malt ; it consists of the soluble parts of this substance dissolved in water. - ED. 
perfect certainty, that ferment is formed from gluten at the same time that the transformation of the sugar is effected; for the wort contains the azotized matter of the corn, namely, gluten in the same condition as it exists in the juice of grapes. The wort ferments by the addition of yeast, but after its decomposition is completed, the quantity of ferment or yeast is found to be thirty times greater than it was originally.

Yeast from beer and that from wine, examined under the microscope, present the same form and general appearance. They are both acted on in the same manner by alkalies and acids, and possess the power of inducing fermentation anew in a solution of sugar; in short, they must be considered as identical.

'The fact that water is decomposed during the putrefaction of gluten has been completely proved. The tendency of the carbon of the gluten to appropriate the oxygen of water must also alway's be in action, whether the gluten is decomposed in a soluble or insoluble state. These considerations, therefore, as well as the circumstance which all the experiments made on this subject appear to point out, that the conversion of gluten to the insoluble state is the result of oxidation, lead us to conclude, that the oxygen consumed in this process is derived from the elements of water, or from the sugar which contains oxygen and hydrogen in the same proportion as water. At all events, the oxygen thus consumed in the fermentation of wine and beer is not taken from the atmosphere.

The fermentation of pure sugar in contact with yeast must evidently be a very different process from the fermentation of wort or must.*

In the former case, the yeast disappears during the decomposition of sugar; but in the latter, a transformation of gluten is effected at the same time,

* The liquid expressed frorn grapes when fully ripe is called must. 
by which ferment is generated. Thus yeast is destroyed in the one case, but is formed in the other.

Now since no free hydrogen gas can be detected during the fermentation of beer and wine, it is evident that the oxidation of the gluten, that is, its conversion into ferment, must take place at the cost either of the oxygen of the water, or of that of the sugar; whilst the hydrogen which is set free must enter into new combinations, or by the deoxidation of the sugar, new compounds containing a large proportion of hydrogen, and small quantity of oxygen, together with the carbon of the sugar, must be formed.

It is well known, that wine and fermented liquors generally contain, in addition to the alcohol, other substances which could not be detected before their fermentation, and which must have been formed, therefore, during that process in a manner similar to the production of mannite. The smell and taste which distinguish wine from all other fermented liquids are known to depend upon an ether of a volatile and highly combustible acid; the ether is of an oily nature, and has received the name Enanthic ether. It is also ascertained, that the smell and taste of brandy from corn and potato are owing to a peculiar oil, the oil of potatoes. This oil is more closely allied to alcohol in its properties, than to any other organic substance.

These bodies are products of the deoxidation of the substances dissolved in the fermenting liquids; they contain less oxygen than sugar or gluten, but are remarkable for the large quantity of hydrogen which enters into their composition.

Enanthic acid contains an equal number of equivalents of carbon and hydrogen, exactly the same proportions of these elements, therefore, as sugar, but by no means the same proportion of oxygen. The oil of potatoes contains much more hydrogen.

Although it cannot be doubted, that these volatile liquids are formed by a mutual interchange of the 
various modifications in the nature of the products generated.

Whatever opinion, however, may be held regarding the origin of the volatile odoriferous substances obtained in the fermentation of wine, it is quite certain that the characteristic smell of wine is owing to an ether of an organic acid, resembling one of the fatty acids (œnanthic ether).

It is only in liquids which contain other very soluble acids, that the fatty acids and œnanthic acids are capable of entering into combination with the ether of alcohol, and of thus producing compounds of a peculiar smell. This ether is found in all wines which contain free acid, and is absent from those in which no acids are present. This acid, therefore, is the means by which the smell is produced; since without its presence œnanthic ether could not be formed.

The greatest part of the oil of brandy made from corn consists of a fatty acid not converted into ether; it dissolves oxide of copper and metallic oxides in general, and combines with the alkalies.

The principal constituent of this oil is an acid identical in composition with œnanthic acid, but different in properties. (Mulder.) It is formed in fermenting liquids, which, if they be acid, contain only acetic acid, a body which has no influence in causing other acids to form ethers.

The oil of brandy made from potatoes is the hydrate of an organic base analogous to ether, and capable, therefore, of entering into combination with acids. It is formed in considerable quantity in fermenting liquids which are slightly alkaline; under circumstances, consequently, in which it is incapable of combining with an acid.

The products of the fermentation and putrefaction of neutral vegetable and animal matters are generally accompanied by substances of an offensive odor; but the most remarkable example of the generation of a true ethereal oil is seen in the fermentation of 
the Herba centaurium minorius, a plant which possesses no smell. When it is exposed in water to a slightly elevated temperature it ferments, and emits an agreeable penetrating odor. By the distillation of the liquid, an ethereal oily substance of great volatility is obtained, which excites a pricking sensation in the eyes, and a flow of tears. (Büchner.)

The leaves of the tobacco plant present the same phenomena; when fresh they possess very little or no smell. When they are subjected to distillation with water, a weak ammoniacal liquid is obtained, upon which a fatty crystallizable substance swims, which does not contain nitrogen, and is quite destitute of smell. But when the same plant, after being dried, is moistened with water, tied together in small bundles, and placed in heaps, a peculiar process of decomposition takes place. Fermentation commences, and is accompanied by the absorption of oxygen; the leaves now become warm and emit the characteristic smell of prepared tobacco and snuff. When the fermentation is carefully promoted and too high a heat avoided, this smell increases and becomes more delicate; and after the fermentation is completed, an oily azotized volatile matter called nicotine is found in the leaves. This substance, - nicotine, which possesses all the properties of a base, was not present before the fermentation. The different kinds of tobacco are distinguished from one another, like wines, by having very different odoriferous substances, which are generated along with the nicotine.

We know, that most of the blossoms and vegetable substances which possess a smell owe this property to a volatile oil existing in them; but it is not less certain, that others emit a smell only when they undergo change or decomposition.

Arsenic and arsenious acid are both quite inodorous. It is only during their oxidation that they emit their characteristic odor of garlic. 'The oil of the berries of the elder-tree, many kinds of oil of turpen- 
tine, and oil of lemons, possess a smell only during their oxidation or decay. The same is the case with many blossoms; and Geiger has shown, that the smell of musk is owing to its gradual putrefaction and decay.

It is also probable, that the peculiar odorous principle of many vegetable substances is newly formed during the fermentation of the saccharine juices of the plants. At all events, it is a fact, that very small quantities of the blossoms of the violet, elder, linden, or cowslip; added to a fermenting liquid, are sufficient to communicate a very strong taste and smell, which the addition of the water distilled from a quantity a hundred times greater would not effect. The various kinds of beer manufactured in Bavaria are distinguished by different flavors, which are given by allowing small quantities of the herbs and blossoms of particular plants to ferment along with the wort. On the Rhine, also, an artificial bouquet is often given to wine for fraudulent purposes, by the addition of several species of the sage and rue to the fermenting liquor; but the fictitious perfume thus obtained differs from the genuine aroma, by its inferior durability, and by being gradually. dissipated.

The juice of grapes grown in different climates differs not only in the proportion of free acid which it contains, but also in respect to the quantity of sugar dissolved in it. The quantity of azotized matter in the juice seems to be the same in whatever parts the grapes rnay grow; at least no difference has been observed in the amount of yeast formed during fermentation in the south of France, and on the Rhine.

The grapes grown in hot climates, as well as the boiled juice obtained from them, are proportionally rich in sugar. Hence, during the fermentation of the juice, the complete decomposition of its azotized matters, and their separation in the insoluble state, are effected before all the sugar has been converted 
into alcohol and carbonic acid. A certain quantity of the sugar consequently remains mixed with the wine in an undecomposed state, the condition necessary for its further decomposition being absent.

The azotized matters in the juice of grapes of the temperate zones, on the contrary, are not completely separated in the insoluble state, when the entire transformation of the sugar is effected. The wine of these grapes, therefore, does not contain sugar, but variable quantities of undecomposed gluten in solution.

'This gluten gives the wine the property of becoming spontaneously converted into vinegar, when the access of air is not prevented. For it absorbs oxygen and becomes insoluble; and its oxidation is communicated to the alcohol, which is converted into acetic acid.

By allowing the wine to remain at rest in casks with a very limited access of air, and at the lowest possible temperature, the oxidation of this azotized matter is effected without the alcohol undergoing the same change, a higher temperature being necessary to enable alcohol to combine with oxygen. As long as the wine in the stilling-casks deposites yeast, it can still be caused to ferment by the addition of sugar, but old well-layed wine has lost this property, because the condition necessary for fermentation, namely, a substance in the act of decomposition or putrefaction, is no longer present in it.

In hotels and other places where wine is drawn gradually from a cask, and a proportional quantity of air necessarily introduced, its eremacausis, that is, its conversion into acetic acid, is prevented by the addition of a small quantity of sulphurous acid. This acid, by entering into combination with the oxygen of the air contained in the cask, or dissolved in the wine, prevents the oxidation of the organic matter.

The various kinds of beer differ from one another in the same way as the wines. 
English, French, and most of the German beers, are converted into vinegar when exposed to the action of air. But this property is not possessed by Bavarian beer, which may be kept in vessels only half-filled without acidifying or experiencing any change. This valuable quality is obtained for it by a peculiar management of the fermentation of the wort. The perfection of experimental knowledge has here led to the solution of one of the most beautiful problems of the theory of fermentation.

Wort is proportionally richer in gluten than in sugar, so that during its fermentation in the common way, a great quantity of yeast is formed as a thick scum. The carbonic acid evolved during the process attaches itself to the particles of yeast, by which they become specifically lighter than the liquid in which they are formed, and rise to its surface. Gluten in the act of oxidation comes in contact with the particles of the decomposing sugar in the interior of the liquid. The carbonic acid from the sugar and insoluble ferment from the gluten are disengaged simultaneously, and cohere together.

A great quantity of gluten remains dissolved in the fermented liquid, even after the transformation of the sugar is completed, and this gluten causes the conversion of the alcohol into acetic acid, on account of its strong disposition to attract oxygen, and to undergo decay. Now, it is plain, that with its separation, and that of all substances capable of attracting oxygen, the beer would lose the property of becoming acid. This end is completely attained in the process of fermentation adopted in Bavaria.

The wort, after having been treated with hops in the usual manner, is thrown into very wide flat vessels, in which a large surface of the liquid is exposed to the air. The fermentation is then allowed to proceed, while the temperature of the chambers in which the vessels are placed is never allowed to rise above from 45 to $50^{\circ} \mathrm{F}$. The fermentation lasts from three to six weeks, and' the carbonic acid 
evolved during its continuance is not in large bubbles which burst upon the surface of the liquid, but in small bubbles like those which escape from a liquid saturated by high pressure. The surface of the wort is scarcely covered with a scum, and all the yeast is deposited on the bottom of the vessel in the form of a viscous sediment.

In order to obtain a clear conception of the great difference between the two kinds of fermentation, it may perhaps be sufficient to recall to mind the fact, that the transformation of gluten or other azotized matters is a process consisting of several stages. The first stage is the conversion of the gluten into insoluble ferment in the interior of the liquid, and as the transformation of the sugar goes on at the same time, carbonic acid and yeast are simultaneously disengaged. It is known with certainty, that this formation of yeast depends upon oxygen being appropriated by the gluten in the act of decomposition; but it has not been sufficiently shown, whether this oxygen is derived from the water, sugar, orfrom the gluten itself; whether it combines directly with the gluten, or merely with its hydrogen, so as to form water. For the purpose of obtaining a definite idea of the process, we may designate the first change as the stage of oxidation. This oxidation of the gluten, then, and the transposition of the: atoms of the sugar into alcohol and carbonic acid, are necessarily attendant on each other, so that if the one is arrested the other must also cease.

Now, the yeast which rises to the surface of the liquid is not the product of a complete decomposition, but is oxidized gluten, still capable of undergoing a new transformation by the transposition of its constituent elements. By virtue of this condition it has the power to excite fermentation in a solution of sugar; and if the gluten be also present, the decomposing sugar induces its conversion into fresh yeast, so that, in a certain sense, the yeast appears: to reproduce itself. 
Yeast of this kind is oxidized gluten in a state of putrefaction, and by virtue of this state it induces a similar transformation in the elements of the sugar.

The yeast formed during the fermentation of $\mathrm{Ba}$ varian beer is oxidized gluten in a state of decay. The process of decomposition which its constituents are suffering, gives rise to a very protracted putrefaction (fermentation) in the sugar. The intensity of the action is diminished in so great a degree, that the gluten which the fluid still holds in solution takes no part in it; the sugar in fermentation does not excite a similar state in the gluten.

But the contact of the already decaying and precipitated gluten or yeast, causes the eremacausis of the gluten dissolved in the wort; oxygen gas is absorbed from the air, and all the gluten in solution is deposited as yeast.

The ordinary frothy yeast may be removed from fermenting beer by filtration, without the fermentation being thereby arrested; but precipitated yeast of Bavarian beer cannot be removed without the whole process of its fermentation being interrupted. The beer ceases to ferment altogether, or, if the temperature is raised, undergoes the ordinary fermentation.

The precipitated yeast does not excite ordinary fermentation, and consequently is quite unfitted for the purpose of baking; but the common frothy yeast can cause the kind of fermentation by which the former kind of yeast is produced.

When common yeast is added to wort at a temperature of between $40^{\circ}$ and $45^{\circ} \mathrm{F}$., a slow tranquil fermentation takes place, and a matter is deposited on the bottom of the vessel, which may be employed to excite new fermentation; and when the same operation is repeated several times in succession, the ordinary fermentation changes into that process by which only precipitated yeast is formed. The yeast now deposited has lost the property of excit- 
ing ordinary fermentation, but it produces the other process even at a temperature of $50^{\supset} \mathrm{F}$.

In wort subjected to fermentation, at a low temperature, with this kind of yeast, the condition necessary for the transformation of the sugar is the presence of that yeast; but for the conversion of gluten into ferment by a process of oxidation, something more is required.

When the power of gluten to attract oxygen is increased by contact with precipitated yeast in a state of decay, the unrestrained access of air is the only other condition necessary for its own conversion into the same state of decay, that is, for its oxidation. We have already seen, that the presence of free oxygen and gluten are conditions which determine the eremacausis of alcohol and its conversion into acetic acid, but they are incapable of exerting this influence at low temperatures. A low temperature retards the slow combustion of alcohol, while the gluten combines spontaneously with the oxygen of the air, just as sulphurous acid does when dissolved in water. Alcohol undergoes no such change at low temperatures, but during the oxidation of the gluten in contact with it, is placed in the same condition as the gluten itself when sulphurous acid is added to the wine in which it is contained. The oxygen of the air unites both with the gluten and alcohol of wine not treated with sulphurous acid; but when this acid is present it combines with neither of them, being altogether absorbed by the acid. The same thing happens in the peculiar process of fermentation adopted in Bavaria. The oxygen of the air unites only with the gluten and not with the alcohol, although it would have combined with both at higher temperatures, so as to form acetic acid.

Thus, then, this remarkable process of fermentation with the precipitation of a mucous-like ferment consists of a simultaneous putrefaction and decay in the same liquid. The sugar is in the state of putrefaction, and the gluten in that of decay. 
Appert's method of preserving food, and this kind of fermentation of beer, depend on the same principle.

In the fermentation of beer after this manner, all the substances capable of decay are separated from it by means of an unrestrained access of air, while the temperature is kept sufficiently low to prevent the alcohol from combining with oxygen. The removal of these substances diminishes the tendency of the beer to become acescent, or, in other words, to suffer a further transformation.

In Appert's mode of preserving food, oxygen is allowed to enter into combination with the substance of the food, at a temperature at which decay, but neither putrefaction nor fermentation, can take place. With the subsequent exclusion of the oxygen and the completion of the decay, every cause which could effect further decomposition of the food is removed. The conditions for putrefaction are rendered insufficient in both cases; in the one by the removal of the substances susceptible of decay, in the other by the exclusion of the oxygen which would effect it.

It has been stated to be uncertain, whether gluten during its conversion into common yeast, that is, into the insoluble state in which it separates from fermenting liquids, really combines directly with oxygen. If it does combine with oxygen, then the difference between gluten and ferment would be, that the latter would contain a larger proportion of oxygen. Now it is very difficult to ascertain this, and even their analyses cannot decide the question. Let us consider, for example, the relations of alloxan and alloxantin* to one another. Both of these bodies contain the same elements as gluten, although in different proportions. Now they are known to be convertible into each other, by oxygen being absorbed in the one case, and in the other extracted. Both

* Products of the decomposition of uric acid by nitric acid, consisting of carbon, nitrogen, hydrogen, and oxygen. "See description, \&c. in WEBSTER's Chemistry, pp. 425 and 430. 
are composed of absolutely the same elements, in equal proportions; with the single exception, that alloxantin contains 1 equivalent of hydrogen more than alloxan.

When alloxantin is treated with chlorine and nitric acid, it is converted into alloxan, into a body, therefore, which is alloxantin minus 1 equivalent of hydrogen. If on the other hand a stream of sulphuretted hydrogen is conducted through alloxan, sulphur is precipitated, and alloxantin produced. It may be said, that in the first case hydrogen is abstracted, in the other added. But it would be quite as simple an explanation, if we considered them as oxides of the same radical: the alloxan being regarded as a combination of a body composed of $\mathrm{C}^{8} \mathrm{~N}^{2} \mathrm{H}^{2} \mathrm{O}^{8}$ with 2 equivalents of water, and alloxantin as a combination of 3 atoms of water, with a compound consisting of $\mathrm{C}^{8} \mathrm{~N}^{2} \mathrm{H}^{2} \mathrm{O}^{7}$. The conversion of alloxan into alloxantin would in this case result from its eight atoms of oxygen being reduced to seven, while alloxan would be formed out of alloxantin, by its combining with an additional atom of oxygen.

Now, oxides are known which combine with water, and present the same phenomena as alloxan and alloxantin. But no compounds of hydrogen are known which form hydrates; and custom, which rejects all dissimilarity until the claim to peculiarity is quite proved, leads us to prefer an opinion, for which there is no further foundation than that of analogy. The woad (Isatis tinctoria) and several species of the Nerium contain a substance similar in many respects to gluten, which is deposited as indigo blue, when an aqueous infusion of the dried leaves is exposed to the action of the air. Now it is very doubtful whether the blue insoluble indigo is an oxide of the colorless soluble indigo, or the latter a combination of hydrogen with the indigo blue. Dumas has found the same elements in both, except that the soluble compound contained 1 equivalent of hydrogen more than the blue. 
In the same manner the soluble gluten may be considered a compound of hydrogen, which becomes ferment by losing a certain quantity of this element when exposed to the action of the oxygen of the air under favorable circumstances. At all events, it is certain that oxygen is the cause of the insoluble condition of gluten ; for yeast is not deposited on keeping wine, or during the fermentation of Bavarian beer, unless oxygen has access to the fluid.

Now, whatever be the form in which the oxygen unites with the gluten, - whether it combines directly with it or extracts a portion of its hydrogen, forming water, - the products formed in the interior of the liquid, in consequence of the conversion of the gluten into ferment, will still be the same. Let us suppose that gluten is a compound of another substance with hydrogen, then this hydrogen must be removed during the ordinary fermentation of must and wort, by combining with oxygen, exactly as in the conversion of alcohol into aldehyde* by eremacausis.

In both cases the atmosphere is excluded; the oxygen cannot, then, be derived from the air, neither can it be supplied by the elements of water, for it is impossible to suppose, that the oxygen will separate from the hydrogen of water, for the purpose of uniting with the hydrogen of gluten, in order again to form water. The oxygen, must, therefore, be obtained from the elements of sugar, a portion of which substance must, in order to the formation of ferment, undergo a different decomposition from that which produces alcohol.' Hence a certain part of the sugar will not be converted into carbonic acid and alcohol, but will yield other products containing less oxygen than sugar itself contains. These products, as has already been mentioned, are the cause of the great

* A liquid having a peculiar ethereal smell, and obtained by passing the vapor of ether through a large glass tube heated to redness, and by other processes. It consists of carbon 4 , hydrogen 4 , oxygen 2 . Its name is from the Latin, alcohol dehydratus. 
difference in the qualities of fermented liquids, and particularly in the quantity of alcohol which they contain.

Must and wort do not, therefore, in ordinary fermentation, yield alcohol in proportion to the quantity of sugar which they hold in solution, a part of the sugar being employed in the conversion of gluten into ferment, and not in the formation of alcohol. But in the fermentation of Bavarian beer, all the sugar is expended in the production of alcohol; and this is especially the case whenever the transformation of the sugar is not accompanied by the formation of yeast.

It is quite certain, that in the distilleries of brandy from potatoes, where no yeast is formed, or only a quantity corresponding to the malt which has been added, the proportion of alcohol and carbonic acid obtained during the fermentation of the mash corresponds exactly to that of the carbon contained in the starch. It is also known, that the volume of carbonic acid evolved during the fermentation of beetroots gives no exact indication of the proportion of sugar contained in them, for less carbonic acid is obtained than the same quantity of pure sugar would yield.

Beer obtained by the mode of fermentation adopted in Bavaria contains more alcohol, and possesses more intoxicating properties, than that made by the ordinary method of fermentation, when the quantities of malt used are the same. The strong taste of the former beer is generally ascribed to its containing carbonic acid in larger quantity, and in a state of more intimate combination; but this opinion is erroneous. Both kinds of beer are; at the conclusion of the fermentation, completely saturated with carbonic acid, the one as much as the other. Like all other liquids, they both must retain such a portion of the carbonic acid evolved as corresponds to their power of solution, that is, to their volumes.

The temperature of the fluid during fermentation 
has a very important influence on the quantity of alcohol generated. It has been mentioned, that the juice of beet-roots allowed to ferment at from $86^{\circ}$ to $95^{\circ}$ ( $30^{\circ}$ to $35^{\circ}$ C.) yields no alcohol; and that afterwards, in the place of the sugar; mannite, a substance incapable of fermentation, and containing very little oxygen, is found, together with lactic acid and mucilage. The formation of these products diminishes in proportion as the temperature is lower. But in vegetable juices, containing nitrogen, it is impossible to fix a limit, where the transformation of the sugar is undisturbed by any other process of decomposition.

It is known, that in the fermentation of Bavarian beer, the action of the oxygen of the air, and the low temperature, cause complete transformation of the sugar into alcohol; the cause which would prevent that result, namely, the extraction of the oxygen of part of the sugar by the gluten, in its conversion into ferment, being avoided by the introduction of oxygen from without.

'The quantity of matters in the act of transformation is naturally greatest at the beginning of the fermentation of must and wort; and all the phenomena which accompany the process, such as evolution of gas, and heat, are best observed at that time. These signs of the changes proceeding in the fluid diminish when the greater part of the sugar has undergone decomposition; but they must cease entirely before the process can be regarded as completed.

The less rapid process of decomposition which succeeds the violent evolution of gas; continues in wine and beer until the sugar has completely disappeared; and hence it is observed, that the specific gravity of the liquid diminishes during many months. This slow fermentation, in most cases, resembles the fermentation of Bavarian beer, the transformation of the dissolved sugar being in part the result of a slow and continued decomposition of the precipita- 
ted yeast; but a complete separation of the azotized substances dissolved in it cannot take place when air is excluded.*

Neither alcohol alone, nor hops, nor indeed both together, preserve beer from becoming acid. The better kinds of ale and porter in England are protected from acidity, but at the loss of the interest of an immense capital. They are placed in large closed wooden vessels, the surfaces of which are covered with sand. In these they are allowed to lie for several years, so that they are treated in a manner exactly similar to wine during its ripening.

A gentle diffusion of air takes place through the pores of the wood, but the quantity of azotized substances being very great in proportion to the oxygen which enters, they consume it, and prevent its union with the alcohol. But the beer treated in this way does not keep for two months without acidifying if it be placed in smaller vessels, to which free access of the air is permitted.

\section{CHAPTER X.}

\section{DECAY OF WOODY FIBRE.}

THE conversion of woody fibre into the substances termed humus and mould is, on account of its influence on vegetation, one of the most remarkable processes of decomposition which occur in nature.

Decay is not less important in another point of

* The great influence which a rational management of fermentation exercises upon the quality of beer is well known in several of the German states. In the grand-duchy of Hesse, for example, a considerable premium is offered for the preparation of beer, according to the Bavarian method; and the premium is to be adjudged to any one who can prove, that the beer brewed by him has lain for six months in the store-vats without becoming acid. Hundreds of casks of beer became changed to vinegar before an empirical knowledge of those conditions was obtained, the influence of which is rendered intelligible by the theory. $-L$. 
view; for, by means of its influence on dead vegetable matter, the oxygen which plants retained during life is again restored to the atmosphere.

The decomposition of woody fibre is effected in three forms, the results of which are different, so that it is necessary to consider each separately.

The first takes place when it is in the moist condition, and subject to free uninterrupted access of air; the second occurs when the air is excluded; and the third when the wood is covered with water, and in contact with putrefying organic matter.

It is known that woody fibre may be kept under water, or in dry air, for thousands of years without suffering any appreciable change; but that when brought into contact with air, in the moist condition it converts the oxygen surrounding it into the same volume of carbonic acid, and is itself gradually changed into a yellowish brown, or black matter, of a loose texture.*

It has already been mentioned, that pure woody fibre contains carbon and the elements of water. Humus, however, is not produced by the decay of pure woody fibre, but by that of wood which contains foreign soluble and insoluble organic substances, besides its essential constituents.

The relative proportions of the component elements are, on this account, different in oak wood and in beech, and the composition of both of these differs very much from woody fibre, which is the same in all vegetables. The difference, however, is so trivial, that it may be altogether neglected in the consideration of the questions which will now be brought under discussion; besides, the quantity of the foreign substances is not constant, but varies according to the season of the year.

* According to the experiments of De Saussure, 240 parts of dry saw-dust of oak wood convert 10 cubic inches of oxygen into the same quantity of carbonic acid, which contains 3 parts, by weight, of carbon; while the weight of the sawdust is diminished by 15 parts. Hence, 12.parts, by weight, of water, are at the same time separated from the elements of the wood. $-L$. 
According to the careful analysis of Gay-Lussac and Thénard, 100 parts of oak wood, dried at $212^{\circ}$ $\left(100^{\circ}\right.$ C. $)$, from which all soluble substances had been extracted by means of water and alcohol, contained 52.53 parts of carbon, and 47.47 parts of hydrogen and oxygen, in the same proportion as they are contained in water.

Now it has been mentioned, that moist wood acts in oxygen gas exactly as if its carbon combined directly with oxygen, and that the products of this action are carbonic acid and humus.

If the action of the oxygen were confined to the carbon of the wood, and if nothing but carbon were removed from it, the remaining elements would necessarily be found in the humus, unchanged except in the particular of being combined with less carbon. The final result of the action would therefore be a complete disappearance of the carbon, whilst nothing but the elements of water would remain.

But when decaying wood is subjected to examination in different stages of its decay, the remarkable result is obtained, that the proportion of carbon in the different products augments. Consequently, if we did not take into consideration the erolution of carbonic acid under the influence of the air, the conversion of wood into humus might be viewed as a removal of the elements of water from the carbon.

The analysis of mouldered oak wood, which was taken from the interior of the trunk of an oak, and possessed a chocolate brown color and the structure of wood, showed that 100 parts of it contained 53.56 parts of carbon and 46.44 parts of hydrogen and oxygen in the same relative proportions as in water. From an examination of mouldered wood of a lightbrown color, easily reducible to a fine powder, and taken from another oak, it appeared that it contained $56 \cdot 211$ carbon and $43 \cdot 789$ water.

These indisputable facts point out the similarity of the decay of wood, with the slow combustion or oxidation of bodies which contain a large quantity 
of hydrogen. Viewed as a kind of combustion, it would indeed be a very extraordinary process, if the carbon combined directly with the oxygen; for it would be a combustion in which the carbon of the burning body augmented constantly, instead of diminishing. Hence it is evident, that it is the hydrogen which is oxidized at the expense of the oxygen of the air; while the carbonic acid is formed from the elements of the wood. Carbon never combines at common temperatures with oxygen, so as to form carbonic acid.

In whatever stage of decay wood may be, its elements must always be capable of being represented by their equivalent numbers.

The following formula illustrates this fact with great clearness :

C 36 H 22 O 22-oak wood, according to Gay-Lussac and Thénard.* C 35 H20 O20 - humus from oak wood (Meyer). $t$

C34 H18018- "6" " (Dr. Will).

It is evident from these numbers, that for every two equivalents of hydrogen which are oxidized, two atoms of oxygen and one of carbon are set free.

Under ordinary circumstances, woody fibre requires a very long time for its decay; but this process is of course much accelerated by an elevated temperature and free unrestrained access of air. The decay, on the contrary, is much retarded by absence of moisture, and by the wood being surrounded with an atmosphere of carbonic acid, which prevents the access of air to the decaying matters.

Sulphurous acid, and all antiseptic substances, arrest the decay of woody fibre. It is well known, that corrosive sublimate is employed for the purpose of protecting the timber of ships from decay; it is a substance which completely deprives vegetable or animal matters, the most prone to decomposition, of

* The calculation gives 52.5 carbon, and 47.5 water.

† The calculation gives 54 carbon, and 46 water.

$\ddagger$ The calculation gives 56 carbon, and 44 water. 
their property of entering into fermentation, putrefaction, or decay.*

But the decay of woody fibre is very much accelerated by contact with alkalies or alkaline earths; for these enable substances to absorb oxygen, which do not possess this power themselves; alcohol, gallic acid, tannin, the vegetable coloring matters, and several other substances, are thus affected by them. Acids produce quite an opposite effect; they greatly retard decay.

Heavy soils, consisting of loam, retain longest the most important condition for the decay of the vegetable matter contained in them, viz., water; but their impermeable nature prevents contact with the air.

In moist sandy soils, particularly such as are composed of a mixture of sand and carbonate of lime, decay proceeds very quickly, it being aided by the presence of the slightly alkaline lime.

Now let us consider the decay of woody fibre during a very long period of time, and suppose that its cause is the gradual removal of the hydrogen in the form of water, and the separation of its oxygen in that of carbonic acid. It is evident, that if we subtract from the formula $\mathrm{C}^{36}, \mathrm{H}^{22}, \mathrm{O}^{22}$, the 22 equivalents of oxygen, with 11 equivalents of carbon, and 22 equivalents of hydrogen, which are supposed to be oxidized by the oxygen of the air, and separated in the form of water; then from 1 atom of oak wood, 25 atoms of pure carbon will remain as the final product of the decay. In other words, 100 parts of oak, which contain 52.5 parts of carbon, will leave as a residue 37 parts of carbon, which must remain unchanged, since carbon does not combine with oxygen at common temperatures.

But this final result is never attained in the decay of wood under common circumstances; and for this reason, that with the increase of the proportion of

* See an account of the process for "kyanizing" timber in the Farm. er's Register, Vol. III. p. 368. 
carbon in the residual humus, as in all decompositions of this kind, its attraction for the hydrogen, which still remains in combination, also increases, until at length the affinity of oxygen for the hydrogen is equalled by that of the carbon for the same element.

In proportion as the decay of woody fibre advances, its property of burning with flame, or in other words, of developing carburetted hydrogen on the application of heat, diminishes. Decayed wood burns without flame; whence no other conclusion can be drawn, than that the hydrogen, which analysis shows to be present, is not contained in it in the same form as in wood.

Decayed oak contains more carbon than fresh wood, but its hydrogen and oxygen are in the same proportion.

We should naturally expect that the flame given out by decayed wood would be more brilliant, in proportion to the increase of its carbon; but we find, on the contrary, that it burns like tinder, exactly as if no hydrogen were present. For the purposes of fuel, decayed or diseased wood is of little value, for it does not possess the property of burning with flame, a property upon which the advantages of common wood depend. The hydrogen of decayed wood must consequently be supposed to be in the state of water; for had it any other form, the characters we have described would not be possessed by the decayed wood.

If we suppose decay to proceed in a liquid, which contains both carbon and hydrogen, then a compound containing still more carbon must be formed, in a manner similar to the production of the crystalline colorless naphthalin from a gaseous compound of carbon and hydrogen. And if the compound thus formed were itself to undergo further decay, the final result must be the separation of carbon in a crystalline form.

Science can point to no process capable of accounting for the origin and formation of diamonds, 
except the process of decay. Diamonds cannot be produced by the action of fire, for a high temperature and the presence of oxygen gas, would call into play their combustibility. But there is the greatest reason to believe that they are formed in the humid way, that is, in a liquid, and the process of decay is the only cause to which their formation can with probability be ascribed.

Amber, fossil resin, and the acids in mellite, are the products of vegetable matter which has suffered decomposition. They are found in wood or brown coal, and have evidently proceeded from the decomposition of substances which were contained in quite a different form in the living plants. They are all distinguished by the proportionally small quantity of hydrogen which they contain. The acid from mellite (mellitic acid) contains precisely the same proportions of carbon and oxygen as that from amber (succinic acid); they differ only in the proportion of their hydrogen. M. Bromeis* found, that succinic acid might be artificially formed by the action of nitric acid on stearic acid, a true process of eremacausis; the experiment was made in this laboratory (Giessen).

\section{CHAPTER XI.}

VEGETABLE MOULD.

The term vegetable mould, in its general signification, is applied to a mixture of disintegrated minerals, with the remains of animal and vegetable substances. It may be considered as earth in which humus is contained in a state of decomposition. Its action upon the air has been fully investigated by Ingenhouss and De Saussure.

When moist vegetable mould is placed in a vessel 
full of air, it extracts the oxygen therefrom with greater rapidity than decayed wood, and replaces it by an equal volume of carbonic acid. When this carbonic acid is removed and fresh air admitted, the same action is repeated.

Cold water dissolves only ${ }_{10,000} \frac{1}{00}$ th of its own weight of vegetable mould; and the residue left on its evaporation consists of common salt with traces of sulphate of potash and limé, and a minute quantity of organic matter, for it is blackened when heated to redness. Boiling water extracts several substances from vegetable mould, and acquires a yellow or yeliowish brown color, which is dissipated by absorption of oxygen from the air,' a black flocculent deposit being formed. When the colored solution is evaporated, a residue is left which becomes black on being heated to redness, and afterwards yields carbonate of potash when treated with water.

A solution of caustic potash becomes black when placed in contact with vegetable mould, and the addition of acetic acid to the colored solution causes no precipitate or turbidness. But dilute sulphuric acid throws down a light flocculent precipitate of a brown or black color, from which the acid can be removed with difficulty by means of water. When this precipitate, after having been washed with water, is brought whilst still moist under a receiver filled with oxygen, the gas is absorbed with great rapidity; and the same thing takes place when the precipitate is dried in the air. In the perfectly dry state it has entirely lost its solubility in water, and even alkalies dissolve only traces of it.

It is evident, therefore, that boiling water extracts a matter from vegetable mould, which owes its solubility to the presence of the alkaline salts contained in the remains of plants. This substance is a product of the incomplete decay of woody fibre. Its composition is intermediate between woody fibre and humus, into which it is converted, by being exposed in a moist condition to the action of the air. 


\section{CHAP'TER XII.}

ON THE MOULDERING OF BODIES. - PAPER, BROWN COAL, AND MINERAL COAL.

The decomposition of wood, woody fibre, and all vegetable bodies when subjected to the action of water, and excluded from the air, is termed mouldering.

Wood, or brown coal and mineral coal, are the remains of vegetables of a former world; their appearance and characters show, that they are products of the processes of decomposition termed decay and putrefaction. We can easily ascertain by analysis the manner in which their constituents have been changed, if we suppose the greater part of their bulk to have been formed from woody fibre.

But it is necessary, before we can obtain a distinct idea of the manner in which coal is formed, to consider a peculiar change which woody fibre suffers by. means of moisture, when partially or entirely excluded from the air.

It is known, that when pure woody fibre, as linen, for example, is placed in contact with water, considerable heat is evolved, and the substance is converted into a soft friable mass, which has lost all coherence. This substance was employed in the fabrication of paper before the use of chlorine, as an agent for bleaching. The rags employed for this purpose were placed in heaps, and it was observed, that on their becoming warm a gas was disengaged, and their weight diminished from 18 to 25 per cent.

When sawdust moistened with water is placed in a closed vessel, carbonic acid gas is evolved in the same manner as when air is admitted. A true putrefaction takes place, the wood assumes a white color, loses its peculiar texture, and is converted into a rotten friable matter. 
The white decayed wood found in the interiors of trunks of dead trees which have been in contact with water, is produced in the way just mentioned.

An analysis of wood of this kind, obtained from the interior of the trunk of an oak, yielded, after having been dried at $212^{\circ}$,

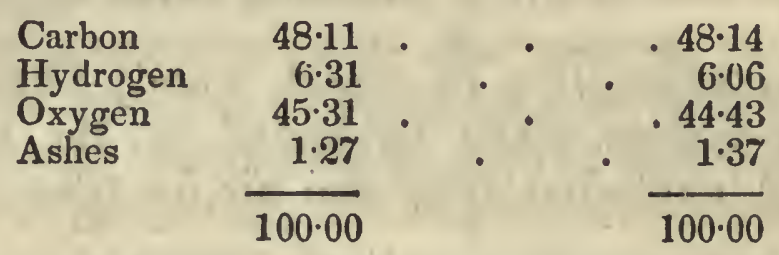

Now, on comparing the proportions obtained from these numbers with the composition of oak wood, according to the analysis of Gay-Lussac and Thénard, it is immediately perceived, that a certain quantity of carbon has been separated from the constituents of wood, whilst the hydrogen is, on the contrary, increased. The numbers obtained by the analysis correspond very nearly to the formula C33 $\mathrm{H} 27$ O24.*

The elements of water have, therefore, become united with the wood, whilst carbonic acid is disengaged by the absorption of a certain quantity of oxygen.

If the elements of 5 atoms of water and 3 atoms of oxygen be added to the composition of the woody fibre of the oak, and 3 atoms of carbonic acid deducted, the exact formula for white mouldered wood is obtained.

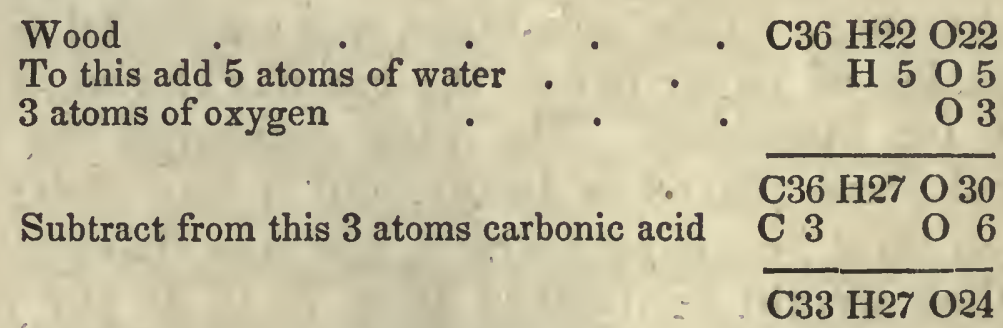

The process of mouldering is, therefore, one of putrefaction and decay, proceeding simultaneously, in which the oxygen of the air and the component

* The calculation from this formula gives in 100 parts 47.9 carbon, $6 \cdot 1$ hydrogen, and 46 oxygen. 
parts of water take part. But the composition of mouldered wood must change according as the access of oxygen is more or less prevented. White mouldered beech-wood yielded on analysis 47.67 carbon, 5.67 hydrogen, and 46.68 oxygen; this corresponds to the formula C33 $\mathrm{H} 25 \mathrm{O} 24$.

The decomposition of wood assumes, therefore, two different forms, according as the access of the air is free or restrained. In both cases carbonic acid is generated; and in the latter case, a certain quantity of water enters into chemical combination.

It is highly probable, that in this putrefactive process, as well as in all others, the oxygen of the water assists in the formation of the carbonic acid.

Wood coal (brown coal of Werner) must have been produced by a process of decomposition similar to that of mouldering. But it is not easy to obtain wood coal suited for analysis; for it is generally impregnated with resinous or earthy substances, by which the composition of those parts which have been formed from woody fibre is essentially changed.

The wood coal, which forms extensive layers in the Wetterau (a district in Hesse Darmstadt), is distinguished from that found in other places, by possessing the structure of wood unchanged, and by containing no bituminous matter. This coal was subjected to analysis, a piece being selected upon which the annual circles could be counted. It was obtained from the vicinity of Laubach; 100 parts contained

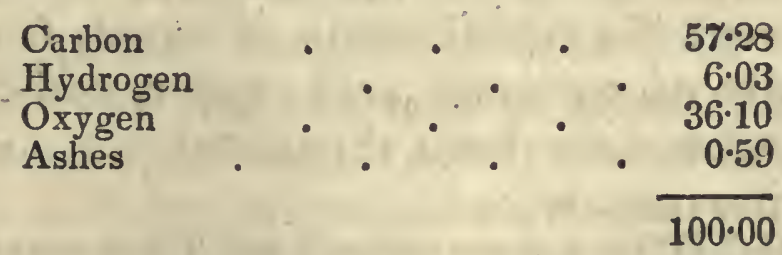

The large amount of carbon, and small quantity of oxygen, constitute the most obvious difference between this analysis and that of wood. It is evident, that the wood which has undergone the change into coal must have parted with a certain portion of 
its oxygen. The proportion of these numbers is expressed by the formula C33 H21 O16.*

When these numbers are compared with those obtained by the analysis of oak, it would appear that the brown coal was produced from woody fibre by the separation of one equivalent of hydrogen, and the elements of three equivalents of carbonic acid.

1 atom wood

Minus 1 atom hydrogen and 3 atoms car-? bonic acid

C36 H22 O22

C 3 H 106

\section{Wood Coal . . $\overline{\text { C33 H21 O16 }}$}

All varieties of wood coal, from whatever strata they may be taken, contain more hydrogen than wood does, and less oxygen than is necessary to form water with this hydrogen; consequently, they must all be produced by the same process of decomposition. The excess of hydrogen is either hydrogen of the wood which has remained in it unchanged, or it is derived from some exterior source. The analysis of wood coal from Ringkuhl, near Cassel, where it is seldom found in pieces with the structure of wood, gave, when dried at $212^{\circ}$,

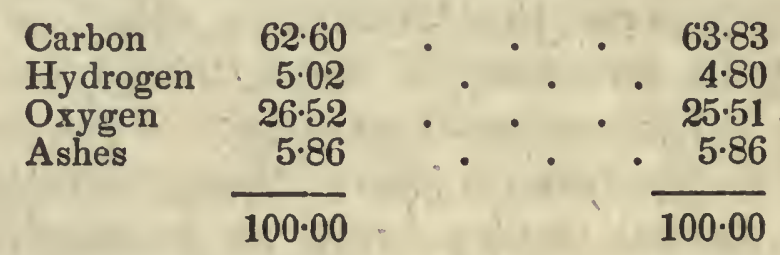

The proportions derived from these numbers correspond very closely to the formula $\mathrm{C}^{32} \mathrm{H}^{15} \mathrm{O}^{9}$, or they represent the constituents of wood, from which the elements of carbonic acid, water, and 2 equivalents hydrogen, have been separated.

C36 H22 $022=$ Wood.

Subtract $\mathrm{C} 4 \mathrm{H} 7 \mathrm{O} 13=4$ atoms carbonic acid +5 atoms of water +2 atoms of hydrogen.

$\overline{\text { C32 H15 O } 9}=$ Wood coal from Ringkuhl.

The formation of both these specimens of wood

* 'The calculation gives 57.5 carbon, and 5.98 hydrogen. 
coal appears from these formulæ to have taken place under circumstances which did not entirely exclude the action of the air, and consequent oxidation and removal of a certain quantity of hydrogen. Now the Laubacher coal is covered with a layer of basalt, and the coal of Ringkuhl was taken from the lowest seam of layers, which possess a thickness of from 90 to 120 feet; so that both may be considered as well protected from the air.

During the formation of brown coal, the elements of carbonic acid have been separated from the wood either alone, or at the same time with a certain quantity of water. It is quite possible, that the difference in the process of decomposition may depend upon the high temperature and pressure under which the decomposition took place. At least, a piece of wood assumed the character and appearance of Laubacher coal, after being kept for several weeks in the boiler of a steam-engine, and had then precisely the same composition. The change in this case was effected in water, at a temperature of from $334^{\circ}$ to $352^{\circ} \mathrm{F}$. $\left(150^{\circ}-160^{\circ}\right.$ C. $)$, and under a corresponding pressure. The ashes of the wood amounted to 0.51 per cent.; a little less, therefore, than those of the Laubacher coal; but this must be ascribed to the peculiar circumstances under which it was formed. The ashes of plants examined by Berthier amounted always to much more than this.

The peculiar process by which the decomposition of these extinct vegetables has been effected, namely, a disengagement of carbonic acid from their substance, appears still to go on at great depths in all the layers of wood coal. At all events it is remarkable, that springs impregnated with carbonic acid occur in many places, in the country between Meissner, in the electorate of Hesse, and the Eifel, which are known to possess large layers of wood coal. These springs of mineral water are produced on the spot at which they are found; the springs of com- 
mon water meeting with carbonic acid during their ascent, and becoming impregnated with it.

In the vicinity of the layers of wood coal at Salzhausen (Hesse Darmstadt) an excellent acidulous spring of this kind existed a few years ago, and supplied all the inhabitants of that district; but it was considered advantageous to surround the sides of the spring with sandstone, and the consequence was, that all the outlets to the carbonic acid were closed, for this gas generally gains access to the water from the sides of the spring. From that time to the present this valuable mineral water has disappeared, and in its place is found a spring of common water.

Springs of water impregnated with carbonic acid occur at Schwalheim, at a very short distance from the layers of wood coal at Dorheim. M. Wilhelmi observed some time since, that they are formed of common spring water which ascends from below, and of carbonic acid which issues from the sides of the spring. The same fact has been shown to be the case in the famed Fachinger spring, by M. Schapper.

The carbonic acid gas from the springs in the Eifel, is, according to Bischoff, seldom mixed with nitrogen or oxygen, and is probably produced in a manner similar to that just described. At any rate the air does not appear to take any part in the formation of these acidulous springs. Their carbonic acid has evidently not been formed either by a combustion at high or low temperatures; for if it were so, the gas resulting from the combustion would necessarily be mixed with $\frac{4}{5}$ of nitrogen, but it does not contain a trace of this element. The bubbles of gas which escape from these springs are absorbed by caustic potash, with the exception of a residuum. too small to be appreciated.

The wood coal of Dorheim and Salzhausen must have been formed in the same way as that of the neighboring village of Laubach; and since the latter contains the exact elements of woody fibre, minus a 
certain quantity of carbonic acid, its composition indicates very plainly the manner in which it has been produced.

The coal of the upper bed is subjected to an incessant decay by the action of the air, by means of which its hydrogen is removed in the same manner as in the decay of wood. This is recognised by the way in which it burns, and by the formation of carbonic acid in the mines.

The gases which are formed in mines of wood coal, and cause danger in their working, are not combustible or inflammable as in mines of mineral coal; but they consist generally of carbonic acid gas, and are very seldom intermixed with combustible gases.

Wood coal from the middle bed of the strata at Ringkuhl gave on analysis $65 \cdot 40,-64.01$ carbon and $4.75,-4.76$ * hydrogen; the proportion of carbon here is the same as in specimens procured from greater depths, but that of the hydrogen is much less.

Wood and mineral coal are always accompanied by iron pyrites (sulphuret of iron) or zinc blende (sulphuret of zinc); which minerals are still formed from salts of sulphuric acid, with iron or zinc, during the putrefaction of all vegetable matter. It is possible, that the oxygen of the sulphates in the layers of wood coal is the means by which the removal of the hydrogen is effected, since wood coal contains less of this element than wood.

According to the analysis of Richardson and Regnault, the composition of the combustible materials in splint coal from Newcastle, and cannel coal from Lancashire, is expressed by the formula C24 H13 O. When this is compared with the composition of woody fibre, it appears that these coals are formed from its elements, by the removal of a certain quantity of carburetted hydrogen and carbonic acid in

* The analysis of brown coal from Ringkuhl, as well as all those of the same substance given in this work, have been executed in this laboratory by $\mathbf{M}$. Kühnert of Cassel. $-L$. 
the form of combustible oils. The composition of both of these coals is obtained by the subtraction of 3 atoms of carburetted hydrogen, 3 atoms of water, and 9 atoms of carbonic acid from the formula of wood.

3 atoms of carburetted hydrogen $\mathrm{C} 3 \mathrm{H} 6$

3 atoms of water . $\mathrm{H} 3 \mathrm{O} 3$

9 atoms of carbonic acid.

C 9018

C36 $\mathrm{H} 22 \mathrm{O} 22=$ wood

Mineral coal $\mid \begin{array}{cc}\text { C24 H13 O } \\ \text { H12 }\end{array}$

Carburetted hydrogen generally accompanies all mineral coal; other varieties of coal contain volatile oils, which may be separated by distillation with water. (Reichenbach.) The origin of naphtha is owing to a similar process of decomposition. Caking coal from Caresfield, near Newcastle, contains the elements of cannel coal, minus the constituents of olefiant gas $\mathrm{C} 4 \mathrm{H} 4$.

The inflammable gases which stream out of clefts in the strata of mineral coal, or in rocks of the coal formations, always contain carbonic acid, according to a recent examination by Bischoff, and also carburetted hydrogen, nitrogen, and olefiant gas; the last of which had not been observed, until its existence in these gases was pointed out by Bischoff. 'The analysis of fire-damp, after it had been treated with caustic potash, showed its constituents to be,

Gas from an

abandoned

mine near

Wallesweiler.

Vol.

Light carburetted hydrogen $91 \cdot 36$

Olefiant gas .

$6 \cdot 32$

Nitrogen gas

$.2 \cdot 32$

$100 \cdot 00$

Gerbard's passage near Luisenthal.

Vol.

83.08

1.98

14.94

$100 \cdot 00$
Gas from a mine near Liekwege.

Vol.

$79 \cdot 10$

$16 \cdot 11$

4.79

$100 \cdot 00$

The evolution of these gases proves, that changes are constantly proceeding in the coal.

It is obvious from this, that a continual removal of oxygen in the form of carbonic acid is effected from layers of 'wood coal, in consequence of which the wood must approach gradually to the composition 
of mineral coal. Hydrogen, on the contrary, is disengaged from the constituents of mineral coal in the form of a compound of carbo-hydrogen; a complete removal of all the hydrogen would convert coal into anthracite.

The formula C36 H22 O22, which is given for wood, has been chosen as the empirical expression of the analysis, for the purpose of bringing all the transformations, which woody fibre is capable of undergoing, under one common point of view.

Now, although the correctness of this formula must be doubted, until we know with certainty the true constitution of woody fibre, this cannot have the smallest influence on the account given of the changes to which woody fibre must necessarily be subjected in order to be converted into wood or mineral coal. The theoretical expression refers to the quantity, the empirical merely to the relative proportion in which the elements of a body are united. Whatever form the first may assume, the empirical. expression must always remain unchanged.

\section{CHAPTER XIII.}

ON POISONS; CONTAGIONS, AND MIASMS.

A GREAT many chemical compounds, some derived' from inorganic nature, and others formed in animals and plants, produce peculiar changes or diseases in the living animal organism. They destroy the vital functions of individual organs; and when their action attains a certain degree of intensity, death is the consequence.

The action of inorganic compounds, such as acids, alkalies, metallic oxides, and salts, can in most cases be easily explained. They either destroy the continuity of particular organs, or they enter into com- 
bination with their substance. The action of sulphuric, muriatic, and oxalic acids, hydrate of potash, and all those. substances which produce the direct destruction of the organs with which they come into contact, may be compared to a piece of iron, which can cause death by inflicting an injury on particular organs, either when heated to redness; or when in the form of a sharp knife. Such substances are not poisons in the limited sense of the word, for their injurious action depends merely upon their condition.

The action of the proper inorganic poisons is owing, in most cases, to the formation of a chemical compound by the union of the poison with the constituents of the organ upon which it acts; it is owing to an exercise of a chemical affinity more powerful than the vitality of the organ.

It is well to consider the action of inorganic substances in general, in order to obtain a clear conception of the mode of action of those which are poisonous. We find that certain soluble compounds, when presented to different parts of the body, are absorbed by the blood, whence they are again eliminated by the organs of secretion, either in a changed or in an unchanged state.

Iodide of potassium, sulpho-cyanuret of potassium, ferro-cyanuret of potassium, chlorate of potash, silicate of potash, and all salts with alkaline bases, when administered internally to man and animals in dilute solutions, or applied externally, may be again detected in the blood, sweat, chyle, gall, and splenic veins; but all of them are finally excreted from the body through the urinary passages.

Each of these substances, in its transit, produces a peculiar disturbance in the organism, -in other words, they exercise a medicinal action upon it, but they themselves suffer no decomposition. If any of these substances enter into combination with any part of the body, the union cannot be of a permanent kind; for their reappearance in the urine shows 
that any compounds thus formed must have been again decomposed by the vital processes.

Neutral citrates, acetates, and tartrates of the alkalies, suffer change in their passage through the organism. Their bases can indeed be detected in the urine, but the acids have entirely disappeared, and are replaced by carbonic acid which has united with the bases. (Gilbert Blane and Wöhler.)

The conversion of these salts of organic acids into carbonates, indicates that a considerable quantity of oxygen must have united with their elements. In order to convert 1 equivalent of acetate of potash into the carbonate of the same base, 8 equivalents of oxygen must combine with it, of which either 2 or 4 equivalents (according as an acid or neutral salt is produced) remain in combination with the alkali ; whilst the remaining 6 or 4 equivalents are disengaged as free carbonic acid. There is no evidence presented by the organism itself, to which these salts have been administered, that any of its proper constituents have yielded so great a quantity of oxygen as is necessary for their conversion into carbonates. Their oxidation can, therefore, only be ascribed to the oxygen of the air.

During the passage of these salts through the lungs, their acids take part in the peculiar process of eremacausis which proceeds in that organ; a certain quantity of the oxygen gas inspired unites with their constituents, and converts their hydrogen into water, and their carbon into carbonic acid. Part of this latter product ( 1 or 2 equivalents) remains in combination with the alkaline base, forming a salt which suffers no further change by the process of oxidation; and it is this salt which is separated by the kidneys or liver.

It is manifest, that the presence of these organic salts in the blood must produce a change in the process of respiration. A part of the oxygen inspired, which usually combines with the constituents of the blood, must, when they are present, combine with 
their acids, and thus be prevented from performing its usual office. The immediate consequence of this must be the formation of arterial blood in less quantity, or in other words, the process of respiration must be retarded.

Neutral acetates, tartrates, and citrates placed in contact with the air, and at the same time with animal or vegetable bodies in a state of eremacausis, produce exactly the same effects as we have described them to produce in the lungs. They participate in the process of decay, and are converted into carbonates just as in the living body. If impure solutions of these salts in water are left exposed to the air for any length of time, their acids are gradually decomposed, and at length entirely disappear.

Free mineral acids, or organic acids which are not volatile, and salts of mineral acids with alkaline bases, completely arrest decay' when added to decaying matter in sufficient quantity; and when their quantity is small, the process of decay is protracted and retarded. They produce in living bodies the same phenomena as the neutral organic salts, but their action depends upon a different cause.

The absorption by the blood of a quantity of an inorganic salt sufficient to arrest the process of eremacausis in the lungs, is prevented by a very remarkable property of all animal membranes, skin, cellular tissue, muscular fibre, \&c.; namely, by their incapability of being permeated by concentrated saline solutions. It is only when these solutions are diluted to a certain degree with water that they are absorbed by animal tissues.

A dry bladder remains more or less dry in saturated solutions of common salt, nitre, ferro-cyanuret of potassium, sulpho-cyanuret of potassium, sulphate of magnesia, chloride of potassium, and sulphate of soda. These solutions run off its surface in the same manner as water runs from a plate of glass besmeared with tallow. 
Fresh flesh, over which salt has been strewed, is found after 24 hours' swimming in brine, although not a drop of water has been added. The water has been yielded by muscular fibre itself, and having dissolved the salt in immediate contact with it, and thereby lost the power of penetrating animal substances, it has on this account separated from the flesh. The water still retained by the flesh contains a proportionally small quantity of salt, having that degree of dilution at which a saline fluid is capable of penetrating animal substances.

This property of animal tissues is taken advantage of in domestic economy for the purpose of removing so much water from meat that a sufficient quantity is not left to enable it to enter into putrefaction.

In respect of this physical property of animal tissues, alcohol resembles the inorganic salts. 'It is incapable of moistening, that is, of penetrating, animal tissues, and possesses such an affinity for water as to extract it from moist substances.

When a solution of a salt, in a certain degree of dilution, is introduced into the stomach, it is absorbed; but a concentrated saline solution, in place of being itself absorbed, extracts water from the organ, and a violent thirst ensues. Some interchange of water and salt takes place in the stomach; the coats of this viscus yield water to the solution, a part of which having previously become sufficiently diluted, is, on the other hand, absorbed. But the greater part of the concentrated solution of salt remains unabsorbed, and is not removed by the urinary passages; it consequently enters the intestines and intestinal canal, where it causes a dilution of the solid substances deposited there, and thus acts as a purgative.

Each of the salts just mentioned possesses this purgative action, which depends on a physical property shared by all of them; but besides this they exercise a medicinal action, because every part of 
the organism with which they come in contact absorbs a certain quantity of them.

The composition of the salts has nothing to do with their purgative action; it is quite a matter of indifference as far as the mere production of this action is concerned (not as to its intensity), whether the base be potash or soda, or in many cases lime and magnesia; and whether the acid be phosphoric, sulphuric, nitric, or hydrochloric.

Besides, these salts, the action of which does not depend upon their power of entering into combination with the component parts of the organism, there is a large class of others which, when introduced into the living body effect changes of a very different kind, and produce diseases or death, according to the nature of these changes, without effecting a visible lesion of any organs.

These are the true inorganic poisons, the action of which depends upon their power of forming permanent compounds with the substance of the membranes, and muscular fibre.

Salts of lead, iron, bismuth, copper, and mercury, belong to this class.

When solutions of these salts are treated with a sufficient quantity of albumen, milk, muscular fibre, and animal membranes, they enter into combination with those substances, and lose their own solubility; while the water in which they were dissolved loses all the salt which it contained.

The salts of alkaline bases extract water from animal substances; whilst the salts of the heavy metallic oxides are, on the contrary, extracted from the water, for they enter into combination with the animal matters.

Now, when these substances are administered to an animal, they lose their solubility by entering into combination with the membranes, cellular tissue, and muscular fibre; but in very few cases can they reach the blood. All experiments instituted for the purpose of determining whether they pass into the urine 
have failed to detect them in that secretion. In fact, during their passage through the organism, they come into contact with many substances by which they are retained.

The action of corrosive sublimate and arsenious acid is very remarkable in this respect. It is known that these substances possess, in an eminent degree, the property of entering into combination with all parts of animal and vegetable bodies, rendering them at the same time insusceptible of decay or putrefaction. Wood and cerebral substance are both bodies which undergo change with great rapidity and facility when subject to the influenee of air and water; but if they are digested for some time with arsenious acid or corrosive sublimate, they may subsequently be exposed to all the influences of the atmosphere without altering in color or appearance.

It is further known, that those parts of a body which come in contact with these substances during poisoning, and which therefore enter into combination with them, do not afterwards putrefy; so that there can be no doubt regarding the cause of their poisonous qualities.

It is obvious, that if arsenious acid and corrosive sublimate are not prevented by the vital principle from entering into combination with the component parts of the body, and consequently from rendering them incapable of decay and putrefaction, they must deprive the organs of the principal property which appertains to their vital condition, viz. that of suffering and effecting transformations; or, in other words, organic life must be destroyed. If the poisoning is merely superficial, and the quantity of the poison so small that only individual parts of the body which are capable of being regenerated have entered.into combination with it, then eschars are produced, - a phenomenon of a secondary kind, - the compounds of the dead tissues with the poison being thrown off by the healthy parts. From these considerations it may readily be inferred, that all internal signs of 
poisoning are variable and uncertain; for cases may happen, in which no apparent indication of change can be detected by simple observations of the parts, because, as has been already remarked, death may occur without the destruction of any organs.

When arsenious acid is administered in solution, it may enter into the blood. If a vein is exposed and surrounded with a solution of this acid, every blood-globule will combine with it, that is, will become poisoned.

The compounds of arsenic, which have not the property of entering into combination with the tissues of the organism, are without influence on life, even in large doses. Many insoluble basic salts of arsenious acid are known not to be poisonous. The substance called alkargen, discovered by Bunsen, has not the slightest injurious action upon the organism; yet it contains a very large quantity of arsenic, and approaches very closely in composition to the organic arsenious compounds found in the body.

These considerations enable us to fix with tolerable certainty the limit at which the above substances cease to act as poisons. For since their combination with organic matters must be regulated by chemical laws, death will inevitably result, when the organ in contact with the poison finds sufficient of it to unite with atom for atom; whilst if the poison is present in smaller quantity, a part of the organ will retain its vital functions.

According to the experiments of Mulder,* the equivalent in which fibrin combines with muriatic acid, and with the oxides of lead and copper, is expressed by the number 6361. It may be assumed, therefore, approximatively, that a quantity of fibrin corresponding to the number 6361 combines with 1 equivalent of arsenious acid, or 1 equivalent of corrosive sublimate.

When 6361 parts of anhydrous fibrin are combined

* Poggendorff's Annalen, Band xl. S. 259. 
with 30,000 parts of water, it is in the state in which it is contained in muscular fibre or blood in the human body. 100 grains of fibrin in this condition would form a neutral compound of equal equivalents with $3 \frac{4}{10}$ grains of arsenious acid, and 5 grains of corrosive sublimate.

The atomic weight of the albumen of eggs and of the blood deduced from the analysis of the compound which it forms with oxide of silver is 7447 , and that of animal gelatin 5652 .

100 grains of albumen containing all the water with which it is combined in the living body, should consequently combine with $1 \frac{1}{4}$ grain of arsenious acid.

These proportions, which may be considered as the highest which can be adopted, indicate the remarkably high atomic weights of animal substances, and at the same time teach us, what very small quantities of arsenious acid or corrosive sublimate are requisite to produce deadly effects.

All substances administered as antidotes in cases of poisoning, act by destroying the power which arsenious acid and corrosive sublimate possess, of entering into combination with animal matters, and of thus acting as poisons. Unfortunately no other body surpasses them in that power, and the compounds which they form can only be broken up by affinities so energetic, that their action is as injurious as that of the above-named poisons themselves. The duty of the physician consists, therefore, in his causing those parts of the poison which may be free and still uncombined, to enter into combination with some other body, so as to produce a compound incapable of being decomposed or digested in the same conditions. Hydrated peroxide of iron is an invaluable substance for this purpose.*

When the action of arsenious acid or corrosive sublimate is confined to the surface of an organ,

* On the preparation, \&c., of this antidote, see Appendix. 
those parts only are destroyed which enter into combination with it; an eschar is formed, which is gradually thrown off.

Soluble salts of silver would be quite as deadly a poison as corrosive sublimate, did not a cause exist in the human body by which their action is prevented, unless their quantity is very great. This cause is the presence of common salt in all animal liquids. Nitrate of silver, it is well known, combines with animal substances, in the same manner as corrosive sublimate, and the compounds formed by both are exactly similar in the character of being incapable of decay or putrefaction.

When nitrate of silver in a state of solution is applied to skin or muscular fibre, it combines with them instantaneously; animal substances dissolved in any liquid are precipitated by it, and rendered insoluble, or, as it is usually termed, they are coagulated. The compounds this formed are colorless, and so stable, that they cannot be decomposed by other powerful chemical agents. They are blackened by exposure to light, like all other compounds of silver, in consequence of a part of the oxide of silver which they contain being reduced to the metallic state. Parts of the body which have united with salts of silver no longer belong to the living organism, for their vital functions have been arrested by combination with oxide of silver; and if they are capable of being reproduced, the neighboring living structures throw them off in the form of an eschar.

When nitrate of silver is introduced into the stomach, it meets with common salt and free muriatic acid; and if its quantity is not too great, it is immediately converted into chloride of silver, - a substance which is absolutely insoluble in pure water. In a solution of salt or muriatic acid, however, chloride of silver does dissolve in extremely minute quantity; and it is this small part which exercises a medicinal influence when nitrate of silver is administered; the remaining chloride of silver is elimi- 
nated from the body in the ordinary way. Solubility is necessary to give efficacy to any substance in the human body.

The soluble salts of lead possess many properties in common with the salts of silver and mercury; but all compounds of lead with organic matters are capable of decomposition by dilute sulphuric acid. The disease called painter's colic is unknown in all manufactories of white lead in which the workmen are accustomed to take as a preservative sulphuric acid-lemonade (a solution of sugar rendered acid by sulphuric acid).

The organic substances which have combined in the living body with metallic oxides or metallic salts, lose their property of imbibing water and retaining it, without at the same time being rendered incapable of permitting liquids to penetrate through their pores. A strong contraction and shrinking of the surface is the general effect of contact with these metallic bodies. But corrosive sublimate, and several of the salts of lead, possess a peculiar property, in addition to those already mentioned. When they are present in excess, they dissolve the first formed insoluble compounds, and thus produce an effect quite the reverse of contraction, namely, a softening of the part of the body on which they have acted.

Salts of oxide of copper, even when in combination with the most powerful acids, are reduced by many vegetable substances, particularly such as sugar and honey, either into metallic copper, or into the red suboxide, neither of which enters into combination with animal matter. It is well known that sugar has been long employed as the most convenient antidote for poisoning by copper.

With respect to some other poisons, namely, hydrocyanic acid and the organic bases strychnia and brucia, we are acquainted with no facts calculated to elucidate the nature of their action. It may, however, be presumed with much certainty, that experiments upon their mode of action on different animal 
substances would very quickly lead to the most satisfactory conclusions regarding the cause of their poisonous effects.

There is a peculiar class of substances, which are generated during certain processes of decomposition, and which act upon the animal economy as deadly poisons, not on account of their power of entering into combination with it, or by reason of their containing a poisonous material, but solely by virtue of their peculiar condition.

In order to attain a clear conception of the mode of action of these bodies, it is necessary to call to mind the cause on which we have shown the phenomena of fermentation, decay, and putrefaction to depend.

This cause may be expressed by the following law, long since proposed by La Place and Berthollet, although its truth with respect to chemical phenomena has only lately been proved. " $A$ molecule set in motion by any power can impart its own motion to another molecule with which it may be in contact."

This is a law of dynamics, the operation of which is manifest in all cases, in which the resistance (force, affinity, or cohesion) opposed to the motion is not sufficient to overcome it.

We have seen that ferment or yeast is a body in the state of decomposition, the atoms of which, consequently, are in a state of motion or transposition. Yeast placed in contact with sugar communicates to the elements of that compound the same state, in consequence of which, the constituents of the sugar arrange themselves into new and simpler forms, namely, into alcohol and carbonic acid. In these new compounds the elements are united together by stronger affinities than they. were in the sugar, and therefore under the conditions in which they were produced further decomposition is arrested.

We know, also, that the elements of sugar assume totally different arrangements, when the substances which excite their transposition are in a different 
state of decomposition from the yeast just mentioned. Thus, when sugar is acted on by rennet or putrefying vegetable juices, it is not converted into alcohol and carbonic acid, but into lactic acid, mannite, and gum.

Again, it has been shown, that yeast added to a solution of pure sugar gradually disappears, but that when added to vegetable juices which contain gluten as well as sugar, it is reproduced by the decomposition of the former substance.

The yeast with which these liquids are made to ferment, has itself been originally produced from gluten.

The conversion of gluten into yeast in these vegetable juices is dependent on the decomposition (fermentation) of sugar; for, when the sugar has completely disappeared, any gluten which may still remain in the liquid does not suffer change from contact with the newly-deposited yeast, but retains all the characters of gluten.

Yeast is a product of the decomposition of gluten; but it passes into a second stage of decomposition when in contact with water. On account of its being in this state of further change, yeast excites fermentation in a fresh solution of sugar, and if this second saccharine fluid should contain gluten, (should it be wort, for example,) yeast is again generated in consequence of the transposition of the elements of the sugar exciting a similar change in this gluten.

After this explanation, the idea that yeast reproduces itself as seeds reproduce seeds, cannot for a moment be entertained.

From the foregoing facts it follows, that a body in the act of decomposition (it may be named the exciter), added to a mixed fluid in which its constituents are contained, can reproduce itself in that fluid, exactly in the same manner as new yeast is produced when yeast is added to liquids containing gluten. This must be more certainly effected when the liquid acted upon contains the body by the met-. 
amorphosis of which the exciter has been originally formed.

It is also obvious, that if the exciter be able to impart its own state of transformation to one only of the component parts of the mixed liquid acted upon, its own reproduction may be the consequence of the decomposition of this one body.

This law may be applied to organic substances forming part of the animal organism. We know that all the constituents of these substances are formed from the blood, and that the blood by its nature and constitution is one of the most complex of all existing matters.

Nature has adapted the blood for the reproduction of every individual part of the organism; its principal character consists in its component parts being subordinate to every attraction. 'These are in a perpetual state of change or transformation, which is effected in the most various ways through the influence of the different organs.

The individual organs, such as the stomach, cause all the organic substances conveyed to them which are capable of transformation to assume new forms. The stomach compels the elements of these substances to unite into a compound fitted for the formation of the blood. But the blood possesses no power of causing transformations; on the contrary, its principal character consists in its readily suffering transformations; and no other matter can be compared in this respect with it.

Now it is a well-known fact, that when blood, cerebral substance, gall, pus, and other substances in a state of putrefaction, are laid upon fresh wounds, vomiting, debility, and at length death, are occasioned. It is also well known, that bodies in anatomical rooms frequently pass into a state of decomposition which is capable of imparting itself to the living body, the smallest cut with a knife, which has been used in their dissection, producing in these cases dangerous consequences. 
The poison of bad sausages belongs to this class of noxious substances. Several hundred cases are known in which death has occurred from the use of this kind of food. In Würtemberg, especially, these cases are very frequent, for there the sausages are prepared from very various materials. Blood, liver, bacon, brains; milk, meal, and bread, are mixed together with salt and spices; the mixture is then put into bladders or intestines, and after being boiled is smoked.

When these sausages are well prepared, they may be preserved for months, and furnish a nourishing, savoury food; but when the spices and salt are deficient, and particularly when they are'smoked too late or not sufficiently, they undergo a peculiar kind of putrefaction, which begins at the centre of the sausage. Without any appreciable escape of gas taking place they become paler in color, and more soft and greasy in those parts which have undergone putrefaction, and they are found to contain free lactic acid, or lactate of ammonia, products which are universally formed during the putrefaction of animal and vegetable matters.

The cause of the poisonous nature of these sausages was ascribed at first to hydrocyanic acid, and afterwards to sebacic acid, although neither of these substances had been detected in them. But sebacic acid is no more poisonous than benzoic acid, with which it has so many properties in common; and the symptoms produced are sufficient to show that hydrocyanic acid is not the poison.

The death which is the consequence of poisoning by putrefied sausages succeeds very lingering and remarkable symptoms. There is a gradual wasting of muscular fibre, and of all the constituents of the body similarly composed; the patient becomes much emaciated, dries to a complete mummy, and finally dies. The carcass is stiff as if frozen, and is not subject to putrefaction. During the progress of the 
disease the saliva becomes viscous and acquires an offensive smell.

Experiments have been made for the purpose of ascertaining the presence of some matter in the sausages to which their poisonous action could be ascribed; but ,no such matter has been detected. Boiling water and alcohol completely destroy the poisonous properties of the sausages, without themselves acquiring similar properties.

Now this is the peculiar character of all substances which exert an action by virtue of their existing condition, - of those bodies the elements of which are in the state of decomposition or transposition; a state which is destroyed by boiling water and alcohol without the cause of the influence being imparted to those liquids; for a state of action or power cannot be preserved in a liquid.

Sausages, in the state here described, exercise an action upon the organism, in consequence of the stomach and other parts with which they come in contact not having the power to arrest their decomposition; and entering the blood in some way or other, while still possessing their whole power, they impart their peculiar action to the constituents of that fluid.

The poisonous properties of decayed sausages are not destroyed by the stomach as those of the smallpox virus are. All the substances in the body capable of putrefaction are gradually decomposed during the course of the disease, and after death nothing remains except fat, tendons, bones, and a few other substances, which are incapable of putrefying in the conditions afforded by the body.

It is impossible to mistake the modus operandi of this poison, for Colin has clearly proved that muscle, urine, cheese, cerebral substance, and other matters, in a state of putrefaction, communicate their own state of decomposition to substances much less prone to change of composition than the blood. When placed in contact with a solution of sugar, 
they cause its putrefaction, or the transposition of its elements into carbonic acid and alcohol.

When putrefying muscle or pus is placed upon a fresh wound, it occasions disease and death. It is obvious that these substances communicate their own state of putrefaction to the sound blood from which they were produced, exactly in the same manner as gluten in a state of decay or putrefaction causes a similar transformation in a solution of sugar.

Poisons of this kind are even generated by the body itself in particular diseases. In small-pox, plague, and syphilis, substances of a peculiar nature are formed from the constituents of the blood. These matters are capable of inducing in the blood of a healthy individual a decomposition similar to that of which they themselves are the subjects; in other words, they produce the same disease. The morbid virus appears to reproduce itself just as seeds appear to reproduce seeds.

The mode of action of a morbid virus exhibits such a strong similarity to the action of yeast upon liquids containing sugar and gluten, that the two processes have been long since compared to one another, although merely for the purpose of illustration. But when the phenomena attending the action of each respectively are considered more closely, it will in reality be seen that their influence depends upon the same cause.

In dry air, and in the absence of moisture, all these poisons remain for a long time unchanged; but when exposed to the air in the moist condition, they lose very rapidly their peculiar properties. In the former case, those conditions are afforded which arrest their decomposition without destroying it; in the latter, all the circumstances necessary for the completion of their decomposition are presented.

The temperature at which water boils, and contact with alcohol, render such poisons inert. Acids, salts of mercury, sulphurous acid, chlorine, iodine, bro- 
mine, aromatic substances, volatile oils, and particularly empyreumatic oils, smoke, and a decoction of coffee, completely destroy their contagious properties, in some cases combining with them or otherwise effecting their decomposition. Now all these agents, without exception, retard fermentation, putrefaction and decay, and when present in sufficient quantity, completely arrest these processes of decomposition.

A peculiar matter to which the poisonous action is due, cannot, we have seen, be extracted from decayed sausages; and it is equally impossible to obtain such a principle from the virus of small-pox or plague, and for this reason, that their peculiar power is due to an active condition recognisable by our senses, only through the phenomena which it produces.

In order to explain the effects of contagious matters, a peculiar principle of life has been ascribed to them, - a life similar to that possessed by the germ of a seed, which enables it under favorable conditions to develop and multiply itself. It would be impossible to find a more correct figurative representation of these phenomena; it is one which is applicable to contagions, as well as to ferment, to animal and vegetable substances in a state of fermentation, putrefaction or decay, and even to a piece of decaying wood, which by mere contact with fresh wood, causes the latter to undergo gradually the same change and become decayed and mouldered.

If the property possessed by a body of producing such a change in any other substance as causes the reproduction of itself, with all its properties, be regarded as life, then, indeed, all the above phenomena may be ascribed to life. But in that case they must not be considered as the only processes due to vitality, for the above interpretation of the expression embraces the majority of the phenomena which occur in organic chemistry. Life would, according to that view, be admitted to exist in every body in which chemical forces act. 
If a body $\mathrm{A}$, for example oxamide (a substance scarcely soluble in water, and without the slightest taste), be brought into contact with another compound B, which is to be reproduced; and if this second body be oxalic acid dissolved in water; then the following changes are observed to take place:The oxamide is decomposed by the oxalic acid, provided the conditions necessary for their exercising an action upon one another are present. The elements of water unite with the constituents of oxamide, and ammonia is one product formed, and oxalic acid the other, both in exactly the proper proportions to combine and form a neutral salt.

Here the contact of oxamide and oxalic acid induces a transformation of the oxamide, which is decomposed into oxalic acid and ammonia. The oxalic acid thus formed, as well as that originally added, are shared by the ammonia, - or in other words, as much free oxalic acid exists after the decomposition as before it, and is of course still possessed of its original power. It matters not whether the free oxalic acid is that originally added, or that newly produced; it is certain that it has been reproduced in an equal quantity by the decomposition.

If we now add to the same mixture a fresh portion of oxamide, exactly equal in quantity to that first used, and treat it in the same manner, the saine decomposition is repeated; the free oxalic acid enters into combination, whilst another portion is liberated. In this manner a very minute quantity of oxalic acid may be made to effect the decomposition of several hundred pounds of oxamide; and one grain of the acid to reproduce itself in unlimited quantity.

We know that the contact of the virus of smallpox causes such a change in the blood, as gives rise to the reproduction of the poison from the constituents of the fluid. This transformation is not arrested until all the particles of the blood which are susceptible of the decomposition have undergone the met- 
amorphosis. We have just seen that the contact of oxalic acid with oxamide caused the production of fresh oxalic acid, which in its turn exercised the same action on a new portion of oxamide. The transformation was only arrested in consequence of the quantity of oxamide present being limited. In their form both these transformations belong to the same class. But no one except a person quite unaccustomed to view such changes will ascribe them to a vital power, although we admit they correspond remarkably to our common conceptions of life; they are really chemical processes dependent upon the common chemical forces.

Our notion of life involves something more than mere reproduction, namely, the idea of an active power exercised by virtue of a definite form, and production and generation in a definite form. By chemical agency we can produce the constituents of muscular fibre, skin, and hair; but we can form by their means no organized tissue, no organic cell.

The production of organs, the coopperation of a system of organs, and their power not only to produce their component parts from the food presented to them, but to generate themselves in their original form and with all their properties, are characters belonging exclusively to organic life, and constitute a form of reproduction independent of chemical powers.

The chemical forces are subject to the invisible cause by which this form is produced. Of the existence of this cause itself we are made aware only by the phenomena which it produces. Its laws must be investigated just as we investigate those of the other powers which effect motion and changes in matter.

The chemical forces are subordinate to this cause of life, just as they are to electricity, heat, mechanical motion, and friction. By the influence of the latter forces, they suffer changes in their direction, an increase or diminution of their intensity, or a complete cessation or reversal of their action. 
Such an influence and no other is exercised by the vital principle over the chemical forces; but in every case where combination or decomposition takes place, chemical affinity and cohesion are in action.

The vital principle is only known to us through the peculiar form of its instruments, that is, through the organs in which it resides. Hence, whatever kind of energy a substance may possess, if it is amorphous and destitute of organs from which the impulse, motion or change proceeds, it does not live. Its energy depends in this case on a chemical action. Light, heat, electricity, or other influences may increase, diminish, or arrest this action, but they are not its efficient cause.

In the same way the vital principle governs the chemical powers in the living body. All those substances to which we apply the general name of food, and all the bodies formed from them in the organism, are chemical compounds. The vital principle has, therefore, no other resistance to overcome, in order to convert these substances into component parts of the organism, than the chemical powers by which their constituents are held together. If the food possessed life, not merely the chemical forces, but this vitality, would offer resistance to the vital force of the organism it nourished.

All substances adapted for assimilation are bodies of a very complex constitution; their atoms are highly complex, and are held together only by a weak chemical action. They are formed by the union of two or more simple compounds; and in proportion as the number of their atoms augments, their disposition to enter into new combinations is diminished; that is, they lose the power of acting chemically upon other bodies.

Their complex nature, however, renders them more liable to be changed, by the agency of external causes, and thus to suffer decomposition. Any external agency, in many cases even mechanical friction, is sufficient to cause a disturbance in the equilibrium 
of the attraction of their constituents; they arrange themselves either into new, more simple, and permanént combinations, or if a foreign attraction exercise its influence upon it, they arrange themselves in accordance with that attraction.

The special characters of food, that is, of substances fitted for assimilation, are absence of active chemical properties, and the capability of yielding to transformations.

The equilibrium in the chemical attractions of the constituents of the food is disturbed by the vital principle, as we know it may be by many other causes. But the union of its elements, so as to produce new combinations and forms, indicates the presence of a peculiar mode of attraction, and the existence of a power distinct from all other powers of nature, namely, the vital principle.

All bodies of simple composition possess a greater or less disposition to form combinations. Thus oxalic acid is one of the simplest of the organic acids, while stearic acid is one of the most complex; and the former is the strongest, the latter one of the weakest, in respect to active chemical character. By virtue of this disposition, simple compounds produce changes in every body which offers no resistance to their action; they enter into combination and cause decomposition.

The vital principle opposes to the continual action of the atmosphere, moisture and temperature upon the organism, a resistance which is, in a certain degree, invincible. It is by the constant neutralization and renewal of these external influences that life and motion are maintained.

The greatest wonder in the living organism is the fact, that an unfathomable wisdom has made the cause of a continual decomposition or destruction, namely, the support of the process of respiration, to be the means of renewing the organism, and of resisting all the other atmospheric influences, such as those of moisture and changes of temperature. 
When a chemical compound of simple constitution is introduced into the stomach, or any other part of the organism, it must exercise a chemical action upon all substances with which it comes in contact; for we know the peculiar character of such a body to be an aptitude and power to enter into combinations and effect decompositions.

The chemical action of such a compound is of course opposed by the vital principle. The results produced depend upon the strength of their respective actions; either an equilibrium of both powers is attained, a change being effected without the destruction of the vital principle, in which case $a$ medicinal effect is occasioned; or the acting body yields to the superior force of vitality, that is, it is digested; or lastly, the chemical action obtains the ascendency and acts as a poison.

Every substance may be considered as nutriment, which loses its former properties when acted on by the vital principle, and does not exercise a chemical action upon the living organ.

Bodies of another class change the direction, the strength, and intensity of the resisting force (the vital principle), and thus exert a modifying influence upon the functions of its organs. They produce a disturbance in the system, either by their presence, or by themselves undergoing a change; these are medicaments.

Compounds of a third class are called poisons, when they possess the property of uniting with organs or with their component parts, and when their power of effecting this is stronger than the resistance offered by the vital principle.

The quantity of a substarce and its condition must obviously completely change the mode of its chemical action.

Increase of quantity is known to be equivalent to superior affinity. Hence a medicament administered in excessive quantity may act as a poison, and a poison in small doses as a medicament. 
Food will act as a poison, that is, it will produce disease, when it is able to exercise a chemical action by virtue of its quantity; or, when either its condition or its presence retards, prevents, or arrests the motion of any organ.

A compound acts as a poison when all the parts of an organ with which it is brought into contact enter into chemical combination with it, while it may operate as a medicine, when it produces only a partial change.

No other component part of the organism can be compared to the blood, in respect of the feeble resistance which it offers to exterior influences. The blood is not an organ which is formed, but an organ in the act of formation; indeed, it is the sum of all the organs which are being formed. The chemical force and the vital principle hold each other in such perfect equilibrium, that every disturbance, however trifling, or from whatever cause it may proceed, effects a change in the blood. This liquid possesses so little of permanence, that it cannot be removed from the body without immediately suffering a change, and cannot come in contact with any organ in the body, without yielding to its attraction.

The slightest action of a chemical agent upon the blood exercises an injurious influence; even the momentary contact with the air in the lungs, although effected through the medium of cells and membranes, alters the color and other qualities of the blood. Every chemical action propagates itself through the mass of the, blood; for example, the active chemical condition of the constituents of a body undergoing decomposition, fermentation, putrefaction, or decay, disturbs the equilibrium between the chemical force and the vital principle in the circulating fluid. Numerous modifications in the composition and condition of the compounds produced from the elements of the blood, result from the conflict of the vital force with the chemical affinity, in their incessant endeavor to overcome one another. 
All the characters of the phenomena of contagion tend to disprove the existence of life in contagious matters. They without doubt exercise an influence very similar to some processes in the living organism; but the cause of this influence is chemical action, which is capable of being subdued by other chemical actions, by opposed agencies.

Several of the poisons generated in the body by disease lose all their power when introduced into the stomach, but others are not thus destroyed.

It is a fact very decisive of their chemical nature and mode of action, that those poisons which are neutral or alkaline, such as the poisonous matter of the contagious fever in cattle (typhus contagiosus ruminantium), or that of the smallpox, lose their whole power of contagion in the stomach; whilst that of sausages, which has an acid reaction, retains all its frightful properties under the same circumstances.

In the former of these cases, the free acid present in the stomach destroys the action of the poison, the chemical properties of which are opposed to it; whilst in the latter it strengthens, or at all events does not offer any impediment to poisonous action.

Microscopical examination has detected peculiar bodies resembling the globules of the blood in malignant putrefying pus, in the matter of vaccine, \&c. The presence of these bodies has given weight to the opinion, that contagion proceeds from the development of a diseased organic life; and these formations have been regarded as the living seeds of disease.

This view, which is not adapted to discussion, has led those philosophers, who are accustomed to search for explanations of phenomena in forms, to consider the yeast produced by the fermentation of beer as possessed of life. They have imagined it to be composed of animals or plants, which nourish themselves from the sugar in which they are placed, and at the 
same time yield alcohol and carbonic acid as excrementitious matters.*

It would perhaps appear wonderful if bodies, possessing a crystalline structure and geometrical figure, were formed during the processes of fermentation and putrefaction from the organic substances and tissues of organs. We know, on the contrary, that the complete dissolution into organic compounds is preceded by a series of transformations, in which the organic structures gradually resign their forms.

Blood, in a state of decomposition may appear to the eye unchanged; and when we recognise the globules of blood in a liquid contagious matter, the utmost that we can thence infer is, that those globules have taken no part in the process of decomposition. All the phosphate of lime may be removed from bones, leaving them transparent and flexible like leather, without the form of the bones being in the smallest degree lost. Again, bones may be burned until they be quite white, and consist merely of a skeleton of phosphate of lime, but they will still possess their original form. In the same way processes of decomposition in the blood may affect individual constituents only of that fluid, which will become destroyed and disappear, whilst its other parts will maintain the original form.

Several kinds of contagion are propagated through the air: so that, according to the view already mentioned, we must ascribe life to a gas, that is, to an aeriform body.

All the supposed proofs of the vitality of contagions are merely ideas and figurative representations, fitted to render the phenomena more easy of apprehension by our senses, without explaining them These figurative expressions, with which we are so willingly and easily satisfied in all sciences, are the foes of all inquiries into the mysteries of nature; they are like the fata morgana, which show us deceitful 
views of seas, fertile fields, and luscious fruits, but leave us languishing when we have most need of what they promise.

It is certain, that the action of contagions is the result of a peculiar influence dependent on chemical forces, and in no way connected with the vital principle. This influence is destroyed by chemical actions, and manifests itself wherever it is not subdued by some antagonist power. Its existence is recognised in a connected series of changes and transformations, in which it causes all substances capable of undergoing similar changes to participate.

An animal substance in the act of decomposition, or a substance generated from the component parts of a living body by disease, communicates its own condition to all parts of the system capable of entering into the same state, if no cause exist in these parts by which the change is counteracted or destroyed.

Disease is excited by contagion.

The transformations produced by the disease assume a series of forms.

In order to obtain a clear conception of these transformations, we may consider the changes which substances, more simply composed than the living body, suffer from the influence of similar causes. When putrefying blood or yeast in the act of transformation is placed in contact with a solution of sugar, the elements of the latter substance are transposed, so as to form alcohol and carbonic acid.

A piece of the rennet-stomach of a calf in a state of decomposition occasions the elements of sugar to assume a different arrangement. The sugar is converted into lactic acid without the addition or loss of any element. ( 1 atom of sugar of grapes C12 $\mathrm{H} 12 \mathrm{O} 12$ yields two atoms of lactic acid $=2$ (C6 H6 06.)

When the juice of onions or of beet-root is made to ferment at high temperatures, lactic acid, mannite, and gum are formed. Thus, according to the differ- 
ent states of the transposition of the elements of the exciting body, the elements of the sugar arrange themselves in different manners, that is, different products are formed.

The immediate contact of the decomposing substance with the sugar is the cause by which its particles are made to assume new forms and natures. The removal of that substance occasions the cessation of the decomposition of the sugar, so that, should its transformation be completed before the sugar, the latter can suffer no further change.

In none of these processes of decomposition is the exciting body reproduced; for the conditions necessary to its reproduction do not exist in the elements of the sugar.

Just as yeast, putrefying flesh, and the stomach of a calf in a state of decomposition, when introduced into solutions of sugar, effect the transformation of this substance, without being themselves regenerated; in the same manner, miasms and certain contagious matters produce diseases in the human organism, by communicating the state of decomposition, of which they themselves are the subject, to certain parts of the organism, without themselves being reproduced in their peculiar form and nature during the progress of the decomposition.

The disease in this case is not contagious.

Now when yeast is introduced into a mixed liquid containing both sugar and gluten, such as wort, the act of decomposition of the sugar effects a change in the form and nature of the gluten, which is, in consequence, also subjected to transformation. As long as some of the fermenting sugar remains, gluten continues to be separated as yeast, and this new matter in its turn excites fermentation in a fresh solution of sugar or wort. If the sugar, however, should be first decomposed, the gluten which remains in solution is not converted into yeast. We see, therefore, that the reproduction of the exciting body here depends, - 
1. Upon the presence of that substance from which it was originally formed;

2. Upon the presence of a compound which is capable of being decomposed by contact with the exciting body.

If we express in the same terms the reproduction of contagious matter in contagious diseases, since it is quite certain that they must have their origin in the blood, we must admit that the blood of a healthy individual contains substances, by the decomposition of which the exciting body or contagion can be produced. It must further be admitted, when contagion results, that the blood contains a second constituent capable of being decomposed by the exciting body. It is only in consequence of the conversion of the second constituent, that the original exciting body can be reproduced.

A susceptibility of contagion indicates the presence of a certain quantity of this second body in the blood of a healthy individual. The susceptibility for the disease and its intensity must augment according to the quantity of that body present in the blood; and in proportion to its diminution or disappearance, the course of the disease will change.

When a quantity, however small, of contagious matter, that is, of the exciting body, is introduced into the blood of a healthy individual, it will be again generated in the blood, just as yeast is reproduced from wort. Its condition of transformation will be communicated to a constituent of the blood; and in consequence of the transformation suffered by this substance, a body identical with or similar to the exciting or contagious matter will be produced from another constituent substance of the blood. The quantity of the exciting body newly produced must constantly augment, if its further transformation or decomposition proceeds more slowly than that of the compound in the blood, the decomposition of which it effects.

If the transformation of the yeast generated in 
the fermentation of wort proceeded with the same rapidity as that of the particles of the sugar contained in it, both would simultaneously disappear when the fermentation was completed. But yeast requires a much longer time for decomposition than sugar, so that after the latter has completely disappeared, there remains a much larger quantity of yeast than existed in the fluid at the commencement of the fermentation, - yeast which is still in a state of incessant progressive transformation, and therefore possessed of its peculiar property.

The state of change or decomposition which effects one particle of blood, is imparted to a second, a third, and at last to all the particles of blood in the whole body. It is communicated in like manner to the blood of another individual, to that of a third person, and so on, - or in other words, the disease is excited in them also.

It is quite certain, that a number of peculiar substances exist in the blood of some men and animals, which are absent from the blood of others.

The blood of the same individual contains, in childhood and youth, variable quantities of substances, which are absent from it in other stages of growth. The susceptibility of contagion by peculiar exciting bodies in childhood, indicates a propagation and regeneration of the exciting bodies, in consequence of the transformation of certain substances which are present in the blood, and in the absence of which no contagion could ensue. The form of a disease is termed benignant, when the transformations are perfected on constituents of the body which are not essential to life, without the other parts taking a share in the decomposition; it is termed malignant when they affect essential organs.

It cannot be supposed, that the different changes in the blood, by which its constituents are converted into fat, muscular fibre, substance of the brain and nerves, bones, hair, \&c., and the transformation of food into blood, can take place without the simulta- 
neous formation of new compounds which require to be removed from the body by the organs of excretion.

In an adult these excretions do not vary much either in their nature or quantity. The food taken is not employed in increasing the size of the body, but merely for the purpose of replacing any substances which may be consumed by the various actions in the organism; every motion, every manifestation of organic properties, and every organic action being attended by a change in the material of the body, and by the assumption of a new form by its constituents.*

But in a child this normal condition of sustenance is accompanied by an abnormal condition of growth and increase in the size of the body, and of each individual part of it. Hence there must be a much larger quantity of foreign substances, not belonging to the organism, diffused through every part of the blood in the body of a young individual.

When the organs of secretion are in proper action, these substances will be removed from the system; but when the functions of those organs are impeded, they will remain in the blood or become accumulated in particular parts of the body. The skin, lungs; and other organs, assume the functions of the diseased secreting organs, and the accumulated substances are eliminated by them. If, when thus exhaled, these substances happen to be in the state of progressive transformation, they are contagious; that is, they are able to produce the same state of disease in another healthy organism, provided the latter organism is susceptible of their action, - or in other words, contains a matter capable of suffering the same process of decomposition.

* The experiments of Barruel upon the different odors emitted from blood on the addition of sulphuric acid, prove that peculiar substances are contained in the blood of different individuals; the blood of a man of a fair complexion and that of a man of dark complexion were found to yield different odors; the blood of animals also differed in this respect yery perceptibly from that of man. $-L$. 
The production of matters of this kind, which render the body susceptible of contagion, may be occasioned by the manner of living, or by the nutriment taken by an individual. A superabundance of strong and otherwise wholesome food may produce them, as well as a deficiency of nutriment, uncleanliness, or even the use of decayed substances as food.

All these conditions for contagion must be considered as accidental. Their formation and accumulation in the body may be prevented, and they may even be removed from it without disturbing its most important functions or health. Their presence is not necessary to life.

The action, as well as the generation of the matter of contagion is, according to this view, a chemical process participated in by all substances in the living body, and by all the constituents of those organs in which the vital principle does not overcome the chemical action. The contagion, accordingly, either spreads itself over every part of the body, or is confined particularly to certain organs, that is, the disease attacks all the organs or only a few of them, according to the feebleness or intensity of their resistance.

In the abstract chemical sense, reproduction of a contagion depends upon the presence of two substances, one of which becomes completely decomposed, but communicates its own state of transformation to the second. The second substance thus thrown into a state of decomposition is the newlyformed contagion.

The second substance must have been originally a constituent of the blood: the first may be a body accidentally present; but it may also be a matter necessary to life. If both be constituents indispensable for the support of the vital functions of certain principal organs, death is the consequence of their transformation. But if the absence of the one substance which was a constituent of the blood do not 
cause an immediate cessation of the functions of the most important organs, if they continue in their action, although in an abnormal condition, convalescence ensues. In this case the products of the transformations still existing in the blood are used for assimilation, and at this period secretions of a peculiar nature are produced.

When the constituent removed from the blood is a product of an unnatural manner of living, or when its formation takes place only at a certain age, the susceptibility of contagion ceases upon its disappearance.

The effects of vaccine matter indicate, that an accidental constituent of the blood is destroyed by a peculiar process of decomposition, which does not affect the other constituents of the circulating fluid.

If the manner in which the precipitated yeast of Bavarian beer acts (page 350 ) be called to mind, the modus operandi of vaccine lymph can scarcely be matter of doubt.

Both the kind of yeast here referred to and the ordinary ferment are formed from gluten, just as the vaccine virus and the matter of smallpox are produced from the blood. Ordinary yeast and the yirus of human smallpox, however, effect a violent tumultuous transformation, the former in vegetable juices, the latter in blood, in both of which fluids respectively their constituents are contained, and they are reproduced from these fluids with all their characteristic properties. The precipitated yeast of Bavarian beer on the other hand acts entirely upon the sugar of the fermenting liquid and occasions a very protracted decomposition of it, in which the gluten which is also present takes no part. But the air exercises an influence upon the latter substance, and causes it to assume a new form and nature, in consequence of which this kind of yeast also is reproduced.

The action of the virus of cow-pox is analogous to that of the low yeast; it communicates its own 
state of decomposition to a matter in the blood, and from a second matter is itself regenerated, but by a totally different mode of decomposition; the product possesses the mild form, and all the properties of the lymph of cow-pox.

The susceptibility of infection by the virus of human smallpox must cease after vaccination, for the substance to the presence of which this susceptibility is owing has been removed from the body by a peculiar process of decomposition artificially excited. But this substance may be again generated in the same individual, so that he may again become liable to contagion, and a second or a third vaccination will again remove the peculiar substance from the system.

Chemical actions are propagated in no organs so easily as in the lungs, and it is well known that diseases of the lungs are above all others frequent and dangerous.

If it is assumed, that chemical action and the vital principle mutually balance each other in the blood, it must further be supposed that the chemical powers will have a certain degree of preponderance in the lungs, where the air and blood are in immediate contact; for these organs are fitted by nature to favor chemical action; they offer no resistance to the changes experienced by the venous blood.

The contact of air with venous blood is limited to a very short period of time by the motion of the heart, and any change beyond a determinate point is, in a certain degree, prevented by the rapid removal of the blood which has become arterialized. Any disturbance in the functions of the heart, and any chemical action from without, even though weak, occasions a change in the process of respiration. Solid substances, also, such as dust from vegetable, animal, or inorganic bodies, act in the same way as they do in a saturated solution of a salt in the act of crystallization, that is, they occasion a deposition 
of solid matters from the blood, by which the action of the air upon the latter is altered or prevented.

-When gaseous and decomposing substances, or those which exercise a chemical action, such as sulphuretted hydrogen and carbonic acid, obtain access to the lungs, they meet with less resistance in this organ than in any other. The chemical process of slow combustion in the lungs is accelerated by all substances in a state of decay or putrefaction, by ammonia and alkalies; but it is retarded by empyreumatic substances, volatile oils, and acids. Sulphuretted hydrogen produces immediate decomposition of the blood, and sulphurous acid combines with the substance of the tissues, the cells, and membranes.

When the process of respiration is modified by contact with a matter in the progress of decay, when this matter communicates the state of decomposition, of which it is the subject, to the blood, disease is produced.

If the matter undergoing decomposition is the product of a disease, it is called contagion; but if it is a product of the decay or putrefaction of animal and vegetable substances, or if it acts by its chemical properties, (not by the state in which it is,) and therefore enters into combination with parts of the body, or causes their decomposition, it is termed miasm.

Gaseous contagious matter is a miasm emitted from blood, and capable of generating itself again in blood.

But miasm properly so called, causes disease without being itself reproduced.

All the observations hitherto made upon gaseous contagious matters prove, that they also' are substances in a state of decomposition. When vessels filled with ice are placed in air impregnated with gaseous contagious matter, their outer surfaces become covered with water containing a certain quantity of this matter in solution. This water soon becomes turbid, and in common language putrefies, 
or, to describe the change more correctly, the state of decomposition of the dissolved contagious matter is completed in the water.

All gases emitted from putrefying animal and vegetable substances in processes of disease, generally possess a peculiar nauseous offensive smell, a circumstance which, in most cases, proves the presence of a body in a state of decomposition. Smell itself may in many cases be considered as a reaction of the nerves of smell, or as a resistance offered by the vital powers to chemical action.

Many metals emit a peculiar odor when rubbed, but this is the case with none of the precious metals, - those which suffer no change when exposed to air and moisturé. Arsenic, phosphorus, musk, the oils of linseed, lemons, turpentine, rue, and peppermint, posséss an odor only when they are in the act of eremacausis (oxidation at common temperatures).

The odor of gaseous contagious matters is owing to the same cause; but it is also generally accompanied by ammonia, which may be considered in many cases as the means through which the contagious matter receives a gaseous form, just as it is the means of causing the smell of innumerable substances of little volatility, and of many which have no odor. (Robiquet.)*

Ammonia is very generally produced in cases of disease ; it is always emitted in those in which contagion is generated, and is an invariable product of the decomposition of animal matter. The presence of ammonia in the air of chambers in which diseased patients lie, particularly of those afflicted with a contagious disease, may be readily detected; for the moisture condensed by ice in the manner just described, produces a white precipitate in a solution of corrosive sublimate, just as a solution of ammonia does. The ammoniacal salts also, which are obtained by the evaporation of rain water after an acid has 
been added, when treated with lime so as to set free their ammonia, emit an odor most closely resembling that of corpses, or the peculiar smell of dunghills.

By evaporating acids in air containing gaseous contagions, the ammonia is neutralized, and we thus prevent further decomposition, and destroy the powser of the contagion, that is, its state of chemical change. Muriatic and acetic acids, and in several cases nitric acid, are to be preferred for this purpose before all others. Chlorine also is a substance which destroys ammonia and organic bodies with much facility; but it exerts such an injurious and prejudicial influence upon the lungs, that it may be classed amongst the most poisonous bodies known, and should never be employed in places in which men breathe.

Carbonic acid and sulphuretted hydrogen, which are frequently evolved from the earth in cellars, mines, wells, sewers, and other places, are amongst the most pernicious miasms. - The former may be removed from the air by alkalies; the latter, by burning sulphur (sulphurous acid), or by the evaporation of nitric acid.

The characters of many organic compounds are well worthy of the attention and study both of physiologists and pathologists, more especially in relation to the mode of action of medicines and poisons.

Several of such compounds are known, which to all appearance are quite indifferent substances, and yet cannot be brought into contact with one another in water without suffering a complete transformation. All substances which thus suffer a mutual decomposition, possess complex atoms; they belong to the highest order of chemical compounds. For example, amygdalin, a constituent of bitter almonds, is a perfectly neutral body, of a slightly bitter taste, and very easily soluble in water. But when it is introduced into a watery solution of synaptas, (a constituent of sweet almonds, ) it disappears completely without the disengagement of any gas, and the wa- 
ter is found to contain free hydrocyanic acid, hydruret of benzule (oil of bitter almonds), a peculiar acid and sugar, all substances of which merely the elements existed in the amygdalin. The same decomposition is effected when bitter almonds, which contain the same white matter as the sweet, are rubbed into a powder and moistened with water. Hence it happens that bitter almonds pounded and digested in alcohol, yield no oil of bitter almonds containing hydrocyanic acid, by distillation with water; for the substance which occasions the formation of those volatile substances, is dissolved by alcohol without change, and is therefore extracted from the pounded almonds. Pounded bitter almonds contain no amygdalin, also, after having been moistened with water, for that substance is completely decomposed when they are thus treated.

No volatile compounds can be detected by their smell in the seeds of the Sinapis alba and S. nigra. A fixed oil of a mild taste is obtained from them by pressure, but no trace of a volatile substance. If, however, the seeds are rubbed to a fine powder, and subjected to distillation with water, a volatile oil of a very pungent taste and smell passes over along with the steam. But if, on the contrary, the seeds are treated with alcohol previously to their distillation with water, the residue does not yield a volatile oil. The alcohol contains a crystalline body called sinapin, and several other bodies. These do not possess the characteristic pungency of the oil, but it is by the contact of them with water, and with the albuminous constituents of the seeds, that the volatile oil is formed.

Thus bodies regarded as absolutely indifferent in inorganic chemistry, on account of their possessing no prominent chemical characters, when placed in contact with one another, mutually decompose each other. Their constituents arrange themselves in a peculiar manner, so as to form new combinations; a complex atom dividing into two or more atoms of 
less complex constitution, in consequence of a mere disturbance in the attraction of their elements.

The white constituents of the almonds and mustard which resemble coagulated albumen, must be in a peculiar state in order to exert their action upon amygdalin, and upon those constituents of mustard from which the volatile pungent oil is produced. If almonds, after being blanched and pounded, are thrown into boiling water, or treated with hot alcohol, with mineral acids, or with salts of mercury, their power to effect a decomposition in amygdalin is completely destroyed. Synaptas is an azotized body which cannot be preserved when dissolved in water. Its solution becomes rapidly turbid, deposits a white precipitate, and acquires the offensive smell of putrefying bodies.

It is exceedingly probable, that the peculiar state of transposition into which the elements of synaptas are thrown when dissolved in water, may be the cause of the decomposition of amygdalin, and formation of the new products arising from it. The action of synaptas in this respect is very similar to that of rennet upon sugar.

Malt, and the germinating seeds of corn in general, contain a substance called diastase, which is formed from the gluten contained in them, and cannot be brought in contact with starch and water, without effecting a change in the starch.

When bruised malt is strewed upon warm starch made into a paste with water, the paste after a few minutes becomes quite liquid, and the water is found to contain, in place of starch, a substance in many respects similar to gum. But when more malt is added and the heat longer continued, the liquid acquires a sweet taste, and all the starch is found to be converted into sugar of grapes.

The elements of diastase have at the same time arranged themselves into new combinations.

The conversion of the starch contained in food into sugar of grapes in diabetes indicates, that amongst 
the constituents of some one organ of the body, a substance or substances exist in a state of chemical action, to which the vital principle of the diseased organ opposes no resistance. The component parts of the organ must suffer changes simultaneously with the starch, so that the more starch is furnished to it, the more energetic and intense the disease must become; while if only food which is incapable of suffering such transformations from the same cause is supplied, and the vital energy is strengthened by stimulant remedies and strong nourishment, the chemical action may finally be subdued, or in other words, the disease cured.

The conversion of starch into sugar may also be effected by pure gluten, and by dilute mineral acids.

From all the preceding facts, we see that very various transpositions, and changes of composition and properties, may be produced in complex organic molecules, by every cause which occasions a disturbance in the attraction of their elements.

When moist copper is exposed to air containing carbonic acid, the contact of this acid increases the affinity of the metal for the oxygen of the air in so great a degree that they combine, and the surface of the copper becomes covered with green carbonate of copper. Two bodies, which possess the power of combining together, assume, however, opposite electric conditions at the moment at which they come in contact.

When copper is placed in contact with iron, a peculiar electric condition is excited, in consequence of which the property of the copper to unite with oxygen is destroyed, and the metal remains quite bright.

When formate of ammonia is exposed to a temper-. ature of $388^{\circ} \mathrm{F} .\left(180^{\circ} \mathrm{C}\right.$.) the intensity and direction of the chemical force undergo a change, and the conditions under which the elements of this compound are enabled to remain in the same form cease to be present." The elements, therefore, arrange 
themselves in a new form; hydrocyanic acid and water being the results of the change.

Mechanical motion, friction, or agitation, is sufficient to cause a new disposition of the constituents of fulminating silver and mercury, that is, to effect another arrangement of their elements, in consequence of which, new compounds are formed.

We know that electricity and heat possess a decided influence upon the exercise of chemical affinity; and that the attractions of substances for one another are subordinate to numerous causes which change the condition of these substances, by altering the direction of their attractions. In the same manner, therefore, the exercise of chemical powers in the living organism is dependent upon the vital principle.

The power of elements to unite together, and to form peculiar compounds, which are generated in animals and vegetables, is chemical affinity; but the cause by which they are prevented from arranging themselves according to the degrees of their natural attractions, - the cause, therefore, by which they are made to assume their peculiar order and form in the body, - is the vital principle.

After the removal of the cause which forced their union, - that is, after the extinction of life, - most organic atoms retain their condition, form, and nature, only by a vis inertia; for a great law of nature proves, that matter does not possess the power of spontaneous action. A body in motion loses its motion only when a resistance is opposed to it; and a body at rest cannot be put in motion, or into any action whatever, without the operation of some exterior cause.

The same numerous causes which are opposed to the formation of complex organic molecules, under ordinary circumstances, occasion their decomposition and transformations when the only antagonist power, the vital principle, no longer counteracts the influence of those causes. Contact with air and the most feeble chemical action now effect changes in the com- 
plex molecules; even the presence of any body the particles of which are undergoing motion or transposition, is often sufficient to destroy their state of rest, and to disturb the statical equilibrium in the attractions of their constituent elements. An immediate consequence of this is, that they arrange themselves according to the different degrees of their mutual attractions, and that new compounds are formed in which chemical affinity has the ascendancy, and opposes any further change, while the conditions under which these compounds were formed remain unaltered. 


\section{APPENDIX TO PART II.}

\section{ANTIDOTE TO ARSENIC.}

The following is from a letter of Samuel L. Dana, M. D., of Lowell, to Dr. Bartlett, published in the "Boston Daily Advertiser." August 3d, 1842.

"According to the experiments of M. Guibourt, white oxide of arsenic, (or white arsenic) digested with hydrated peroxide of iron, forms a compound, whose proportions differ from that of arsenite of iron, by containing a larger portion of iron. It is this salt, which forms in the stomach, when peroxide of iron is administered as an antidote to arsenic. It contains $3 \frac{1}{2}$ times as much iron as arsenic. It is perfectly insoluble and innocuous. Three things are essential to the action of this antidote.

" 1 st. Perfect freedom from protoxide of iron.

" 2 d. Perfect freedom from free alkali, or alkali combined with the oxide of iron.

" 3 d. It must be freshly prepared without drying.

" 1 st. If the antidote contains protoxide of iron, then that combines with the arsenic and forms a compound which, though of sparing solubility, is yet poisonous and prevents the ulterior good action of the peroxide of iron. A mixture of prot and peroxides of iron is no antidote to arsenic.

" $2 \mathrm{~d}$. If carbonate of potash is used to precipitate a solution of persalt of iron, a portion falls, combined with alkali. Hence Berzelius recommends bicarbonate of potash, cold, to be used for this purpose. The effect of alkali, free, or thus combined with peroxide of iron, will be, to form soluble poisonous arsenites as above noticed.

" $3 \mathrm{~d}$. The effect depends on the antidote being freshly prepared. I would therefore, in order to insure the $2 \mathrm{~d}$ and $3 \mathrm{~d}$ conditions, recommend the solution of pernitrate of iron to be taken dilute, followed by aq. am. and wet by a 
little vinegar or tartaric acid, or cream of tartar; remedies always at hand.

"To insure perfect freedom from protoxide of iron, I would always pass a current of chlorine, through the solution of prepared nitrate of iron, before that is considered as fit, to be kept on hand, for the ready formation of hydrated peroxide of iron.

\section{T A B L E S :}

SHOWING THE PROPORTION BETWEEN THE HESSIAN AND ENGLISH STANDARD OF WEIGHTS AND MEASURES.

In general all the weights and measures employed in this edition are those of the English standard. In a few cases only, the Hessian weights and measures have been retained. In these the numbers do not represent absolute quantites, but are merely intended to denote a proportion to other numbers. This has been done to avoid any unnecessary intricacy in the calculations, and to present whole numbers to the reader, without distracting his attention by decimal parts. For those, however, who wish to be acquainted with the exact English quantities, a table is here given below.

$1 \mathrm{lb}$. English is equal to $0.90719 \mathrm{lb}$. Hessian; hence, about one-tenth less than the latter.

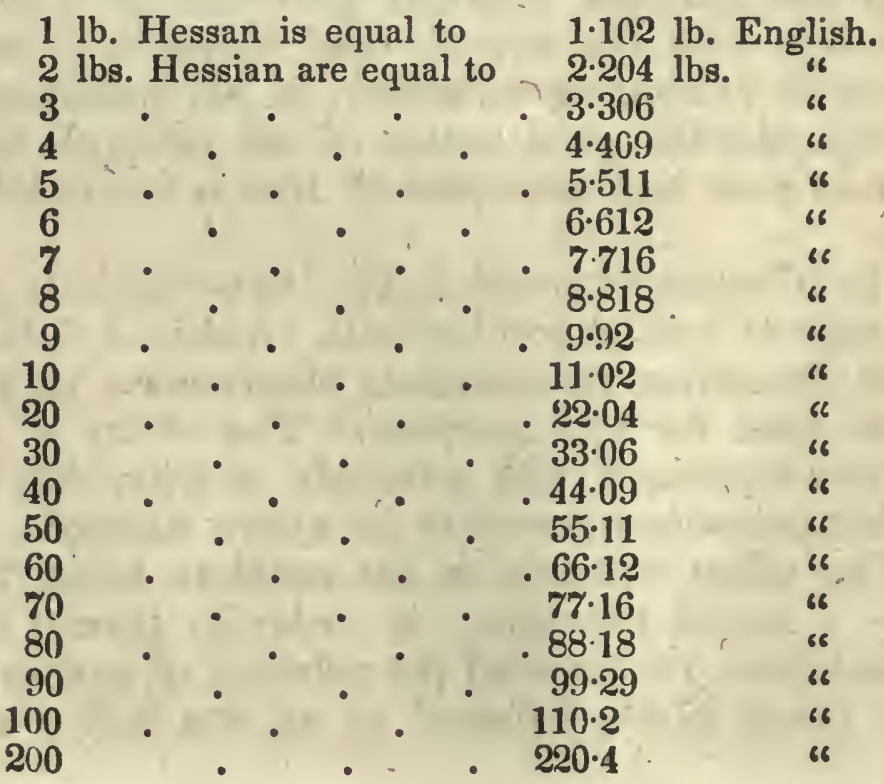


TABLE OF THE CORRESPONDING DEGREES ON THE SCALES OF FAHRENHEIT, REAUMUR, AND CELSIUS, OR CENTIGRADE.

\begin{tabular}{|c|c|c|c|c|c|c|c|c|}
\hline 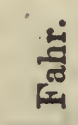 & تِّ & $\begin{array}{l}\overrightarrow{0} \\
0 \\
\tilde{J}\end{array}$ & 壱 & 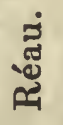 & $\begin{array}{l}\overrightarrow{0} \\
\stackrel{0}{0} \\
\end{array}$ & 丞 & 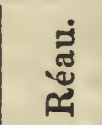 & نٍ \\
\hline 212 & 80 & 100 & 149 & 52 & 65 & 50 & 8 & 10 \\
\hline 20 & 76 & 95 & & 48 & 60 & 41 & 4 & \\
\hline 19 & 72 & 90 & & 44 & 5 & & 0 & 0 \\
\hline 185 & 68 & 85 & 122 & 40 & 50 & 23 & & -5 \\
\hline 176 & 64 & 80 & 11 & 36 & 45 & 14 & -8 & -10 \\
\hline 167 & 60 & 75 & 104 & 32 & 40 & 5 & -12 & -15 \\
\hline 158 & 56 & 70 & 95 & 28 & 35 & 4 & -16 & -20 \\
\hline & & & 8 & 24 & 30 & $-13^{*}$ & -20 & -25 \\
\hline & & & 7 & 20 & 25 & -22 & -24 & -30 \\
\hline & & & & 16 & 20 & -31 & -28 & -35 \\
\hline & & & & 12 & 15 & -40 & -32 & -40 \\
\hline
\end{tabular}

* - Denotes below the cipher on Fahrenheit's scale. 


\section{INDEX.}

ACI

ALK

ABNORMAL, meaning of the term, 140.

Absorption, by roots, 107 .

Of salts, 116.

Acetone, 306.

Acid, acetic, emitted by plants, 150 .

- compound atom of, 301.

transformation of, 306.

formation of, $329-334$.

Apocrenic, 31 .

Boracic, 122.

Carbonic, 24 - 70.

sphere, 28.

- decomposed by plants, 43. from respiration, 44. from springs, 29.

plants, 105.

why necessary to

Crenic, 31.

Cyanic, transformation of, 310 .

Cyanuric, 70.

Formic, 71, 86, 290.

Hippuric, 97.

Humic, 31 .

- properties of, 34 .

Hydrocyanic, 70, 290.

Hydromellonic, 70 .

Hy pochlorous, 293.

Kinic, 114.

Kinovic, 301.

Lactic, 190.

production of, 321.

Meconic, 115.

Melanic, 326.

Mellitic, 363.

Nitric, source of, 88.

Oxalic, 70.

Phosphoric, in ashes of plants, 155.

Rocellic, in plants, 108.
Acid, succinic, 363 .

Sulphuric, action of, on soils, $208,248$.

Tartaric, in grapes, 108.

Acids, action of upon sugar, 303 .

Arrest decay, 361.

Capacity for saturation, 108.

Organic, in plants, 27, 107.

Acre, Hessian, 36.

Adipocire, 88.

Affinity, action of, 71 .

Chemical, examples of, 292.

Weak, example of, 293.

Agave Americana, absorbs oxygen, 51.

Agriculture, in China, 193.

Object of, 100, 145, 172.

how attained, 146.

Its importance, $\mathbf{1 4 3 .}$

A principle in, 187.

Air, access of, favored, 65.

Ammonia in, 29, 91.

Carbonic acid in, 41.

Effect of upon juices, 330 . on soils, 167.

Expired in phthisis, 73.

Improved by plants, 47.

Necessary to plants, 130 .

Albumen, 96, contains nitrogen, 27.

Alcohol, effect of heat on, 306 .

Exhaled, 72.

Products of its oxidation, 327.

From sugar, 313.

Aldehyde, 327.

Alkalies, 69 , from granitic soils, 117 .

Presence of, indicated, 215.

Promote decay of wood, 361.

Quantity in aluminous minerals, 148.

Alkaline Bases, in plants, on what their existence depends, 112. 
Alkaline Bases, salts contained in fertile soils, 153.

Salts in plants, sources of, 151. Allantoin, 70. Alloxan, 352.

Alloxantin, 352.

Alumina, in fertile soils, 147.

Its influence on vegetation, 147.

Mistaken in ashes, 148.

Amber, origin of, 363 .

Ammelin, 70 .

Ammonia, 70, 86, carbonate of, from urine, 191.

Chow fixed, 191.

Cause of nitrification, 338 .

Changes colors, 87.

Condensed by charcoal, 104.

Conversion of, into nitric acid, 338.

Decomposition of by plants, 266 .

Early existence of, 123.

Fixed by gypsum, 191.

From animals, 174.

Contained in beet-root, \&c., 93.

— maple juice, 94 .

Furnishes nitrogen, 104.

Loss from evaporation, 99.

- prevented, 280.

Produced by animal organism, 123.

Product of decay, 88.

Properties of, 88 .

Quantity absorbed by charcoal, 104.

wood, 104.

In rain water, 90 .

How detected, 91.

Separated from soils by rain; 104 .

In snow water, 91.

Solubility of, 89.

Sulphate of, 281.

Transformation of, 86 .

Ammoniacal Liquor, 283.

Amylin, its effect, $\boldsymbol{\gamma} 4$.

Analysis of decayed wood, 359.

Of fire-damp, 372.

Of fishes, 177.

Of horse-dung, 177.

Of peat, 185.

Of guano, 201.

Of lentils, 159.

Of oak-wood, 358.

Of night.soil, 179.

Of salt water, 124.
Analysis, of soils, 217, 245.

Of wood coal, 367,368 .

Animal food, preservation of, 330 .

Life, connexion of, with plants, 22.

Bodies, products of decay, 88 . complex, 302.

Animals, excrements of, 189.

Nutriment of, 22.

Annual plants, how nourished, 135. Anthoxanthum Odoratum, acid in, 97.

Anthracite, 373.

Antidotes to Poisons, 381.

Apatite, 156.

Apotheme, $3 \mathrm{~L}$.

Arable Land, 146.

Aromatics, their influence on fermentation, 343.

Argillaceous Earth, its origin, 147. Arrugonite, transformation of, 298. Arrow Root, 140.

Arsenious Acid, action of, 381 .

Artificial Manure, 199, 287.

Ashes, as manure, 182, 198.

Comparative value of, 182.

Of fir-wood, 111.

Of pine trees, 110.

Of plants, origin of salt in, 125 .

Importance of examination of, 112.

Of wheat, 158.

Of bones, 183 .

Of peat, 185 .

Of coals, 198.

Phosphate of lime in, 183.

Assimilation, of carbon, 30 .

Of carbonic acid, and ammonia, 131.

Of hydrogien, $80-84$.

Of nitrogen, 85-105.

Its power, 140 .

Atmosphere, ammonia in, 29, 92.

'Composition of, 27.

How maintained, 44.

Composition is invariable, 40.

Carbonic acid in the, 28-41.

Motion of, 46.

Oxygen in, 26.

Atoms, motions of, 297.

Permanence in position of, 297.

Attraction, powerful, overcome, 309 .

Azores, glairin found there, 34 .

Carbonic acid at the, 79.

Silica in hot springs of, 170 . 
Azotized matter in juices of plants, 137.

Substances, combustion of, 334 . Azulmin, 70 .

B.MB OO, silica in, 171.

Bark of trees, products in, 49 .

Barilla, 118.

Barley, analysis of, 155 .

Barruel, his experiments on the blood, 403.

Base, what; 69, 106.

Bases, alkaline, in plants, on what their existence depends, 112.

\section{Organic, 27.}

Oxygen contained in, 106.

In plants, 108.

Substitution of, 109.

Beans, alkalies in, 159.

Nutritive power of, 159 .

Becquerel, experiments of, 150 .

Beech, ashes of, 182.

Beer, 347 - 357 .

Bavarian, 348.

Varieties of, 347.

Beet-root sugar, 38.

Ammonia from, 93.

From sandy soils, 140.

Belgium, soils of, 241.

Benignant Disease, 402.

Benzoic acid, formed, 97.

Berzelius, humic extract of, 34 .

His analysis of bones, 158 .

Birch Tree, ammonia from, 94.

Bischoff, estimate of carbonic acid, \&c., 29.

Bluke, on nitrate of soda, 270.

Bleaching Salts, 141.

Blood, its office, 135.

Action of chemical agents upon, 396.

Its feeble resistance to exterior influences, 396.

Organic salts in, 375.

Its character, 386.

Blossoms, when produced, 68 .

Increased, 132.

Removal of, from potatoes, 134.

Bones, dust of, 183.

Durability of, 204.

Gelatine in, 203.

Use in composts, 212.

Composition of, 157, 158.

Bouquet of wines, 342.

Boracic Acid, 122.

Botanists, neglect of chemistry by, 55.
Bran, use of, 185.

Brandy, from corn, 342.

Oil of, 342.

Brazil, wheat in, 153.

Bread, from wood, 133.

Brown Coal, 185.

Buckrcheat, ashes of, 159.

Bulbs, how nourished, 76.

CALCAREOUS SPAR, 298.

Calcium, fluoride of, 157.

Chloride of, 192.

Calculous Disorders, 74.

Calico Printing, use of cow-dung in, 186.

Use of phosphate of soda in, 286.

Substitute for, 186, 286.

Caoutchouc, in plants, 78.

Carbon, 24.

Afforded to the soil by plants, 76 .

Assimilation of, $30-63$.

Combination of, with oxygen, 24 .

Of decaying substances seldom affected by oxygen, 360 .

Derived from air, 44 .

In decaying wood, 360 .

In decaying woody fibre, 361 .

In sea-water, 45 .

Oxide of, formed, 305.

Quantity in grain, 38.

in land, 39.

in straw, 38.

Restored to the soil, 76 .

Received by leaves, 43 .

Its affinity for oxygen, 328.

Carbonate of ammonia decomposed by gypsum, 100.

Of soda, 207.

Of lime in caverns and vaults, 128.

Carbonic acid, 70, in the atmosphere, 28.

In St. Michaels, 79.

Changes in leaves, 142.

Decomposed by plants, 43.

Emission of, at night, 49.

Evaporation of, 56 .

Evolution from decaying bodies, 328.

From decaying plants, 84.

- excrements, 99.

- humus, 65.

— respiration, 72.

- springs, 29, 85.

- woody fibre, 64 .

Quantity extracted from air, 45. 
Carbonic Acid, influence of light on its decomposition, 53.

Increase of, prevented, 42.

Carbon of Plants, source of, 260 285.

Carburetted hydrogen with coal, 372.

Caverns, stalactites in, 127.

Charcoal, what, 24.

Condenses ammonia, 104.

Experiments of Lucas on, 249.

May replace humus, 78.

Theory of its action, 78 .

Promotes growth of plants, 249.

Chelmsford, analysis of soil of, 246.

Chemical effects of light, 141.

Forces can replace the vital prin. ciple, 75.

Processes in nutrition of vegetables, 22.

Transformations, 69, 289.

Chemistry, definition of, 21 .

Organic, what is, 22 .

Neglected by botanists, 55 ; and physiologists, 56 .

China, its agriculture, 193.

Collection and use of manure in, 193.

Chlorine gas, 141 : effect of, 101.

Chloride of calcium, $19 \%$.

Of nitrogen, 293.

Of potassium, its effect, 116 .

Of sodium, its volatility, 123.

Clay, burned, advantages of, as a manure, 102.

Clays, potash in, 148.

Clay slate, 157.

Coal, formation of, 369.

Ammoniacal liquor from, 205.

Inflammable gases from, 372.

Origin of substances in, 363.

Of humus, 30, 129.

Wood or brown, 185.

Colors of flowers, 96.

Combustion at low temperatures, 327.

Of decayed wood, 362 .

Induction of, 332.

Removes oxygen, 42.

Spontaneous, 324 .

Compost manure, 118, 212, 279.

Concretions from horses, 156.

Constituents of plants, 24.

Consumption, 73 .

Contagion, reproduction of, on what dependent, 389.
Contagion, susceptibility to, how occasioned, $4(1) 1$.

Contagions, how produced, 389.

Propagation of, 398.

Contagious matters, action of, 394 , $399,413$.

Their effects explained, 390 .

Life in, disproved, 392.

Reproduction of, 392.

Copper alloy, its action, on sulphu. ric acid, 292.

Corn, how cultivated in Italy, 152

Phosphate of magnesia in, 156.

Effect of carbonic acid on, 79.

Corn brandy, 342 .

Corrosive sublimate, action of, 381 .

Cowo, excrements of the, 120,176 , 178.

Variable in value, 179.

Urine of the, 177; rich in potash, 119.

Coro-pox, action of virus of, 405 .

Crops, rotation of, 161 .

Favorable effects of, 16 ?.

Principles regulating, 174, 275.

Cubic nitre, 270.

Cultivation, its benefits, 47 .

Different methods of, 144 .

Object of, 145.

Culture, art of, 126.

Of plants, principles of the, 144 .

Cyanic acid, transformation of, 311. Cyanogen, combustion of, 335 .

A compound base, 70 .

Transformation of, 311.

Cyanuric acid, 70.

D.ANA, Dr. S. L., on geine, 31.

On phosphate of lime, 182.

On ammonia, 259.

On phosphate of soda in calico printing, 286.

Daniel's manure, $28 \%$.

Darroin, on nitrate of soda, 270 .

Daubeny, experiments of, 105 .

On forest trees, 164.

On nutritive qualities of plants, 265.

On source of carbon, 285.

On source of carbon of plants, 260.

On source of hydrogen of plants; 263.

Carbon of, 260.

Experiments at Oxford, 257.

Experiments on his farm, 273.

Source of hydrogen, 263. 
Davis, his account of Chinese manure, 193.

Death from nutritious substances, 59.

The source of life, 105.

Decundolle, his theory of excretion, 163.

Difference of his views and those of Macaire-Princep, 167.

Decay, 292.

A source of ammonia, 88 .

Of wood, 361 .

Of plants restores oxygen, 84 . and putrefaction, 291 .

Decomposition, 68, 289.

Organic, chemical, 291.

Dextrine, $56,57$.

Diamond, its origin, 363.

Diastase, 136.

Contains nitrogen, 136.

Disease, how excited, 386.

Dog, excrement of the, 175.

Dung hills, liquid from, 191 .

Reservoirs, 191.

Substitute, 187.

EBONY WOOD, oxygen and hydrogen in, 53.

Effete matters separated, 68.

Eifel, springs evolve carbonic acid, 29.

Elements of plants, 24

Not generated by organs, 59 .

Elphinstone, Sir Honoard, on sodaash as a manure, 207.

England, analysis of soils in, 242. Equilibrium of attractions disturbed, 298.

Equisetacece contain silica, 171 .

Eremacausis, 63, 299.

Analogous to putrefaction, 328.

Arrested, 323 .

Definition of, 299.

Necessary to nitrification, 335 .

Of bodies containing nitrogen, 334.

Of bodies destitute of nitrogen, 329.

Ether, œnanthic, 344.

Etiolation, 46.

Eudiometer, 90.

Excrementitious matter, production of, illustrated, 71 .

Excrement, animal, its chemical nature, 175.

Of the dog, cow, \&c., 175.

Influence of, as mianure, 180.
Excréments of plants, 163.

Conversion of, into humus, 35.

Of man, amount of, 195.

Value of, 189.

Preservation of, 193.

Excretion, organs of, 72.

Of plants, theory of, 163.

Experiments in physiology, object of, 56.

Of physiologists not satisfactory, 61.

Extract of humus, 31.

FALLOW, changes from, 152.

Crops, 159.

Time, 159.

Fattening of animals, 146.

Faces, analysis of, 179.

Ferment, 313, :314.

Fermentation, 299, 300.

Causes of, 292.

Of Bavarian beer, 348.

Of beer, 349.

Gay-Lussac's experiments in, 330.

Of sugar, 313.

Of vegetable juices, 314 .

Vinous, 338.

Of wort, 339.

Fertility of fields, how preserved, 181.

Fires, plants on localities of, 154 .

Firs, succeed oaks, 164.

Fir-roood, analysis of its ashes, 111.

Fishes, in salt-pans, 121.

As manure, 259

Flanders, manure in, 193.

Fleabane, 160.

Flesh, composition of, 177.

Effect of salt on, 377 .

Flour, bran of, 185.

Flowers, colors due to ammonia, 96 .

Fluorine, 157; in ancient bones, 158.

Foliage, increased, 101.

Food, effect on products of plants, 139.

Of young plants, $\mathbf{J} 31$.

Transformation and assimilation. of, 72.

Formation of wood, 138.

Formic acid, 70, 290.

Theory of its formation, 71 .

From hydrocyanic acid, 71 .

Fossil resin, origin of, 363 .

Franconia, caverns in, 127.

Fruit, increased, 132. 
Fruit, ripening of, 83 .

132.

Fulminating silver, 233.

GASEOUS substances in the lungs, effect of, 407.

Gasterosteus aculeatus, in salt-pans, 121.

Gasworks, liquor of, 205, 283.

Gay-Lussac, his experiments, 330.

Geine, 31 .

Germany, cultivation in, 181 .

Germination of potatoes, 133.

Of grain, 137.

Glairin, 34 .

Glass, as a manure, 187.

Effect of heat on, 297.

Glue, manure from, 184.

Gluten, conversion of, into yeast, $348-356$.

Decomposition of, 321.

Gas from, 339.

Grain, germination of, 137 .

Manure for, 119.

Rust in, 220.

Granitic, soil affords alkalies, 117.

Grapes, fermentation of, 338 .

Juice of, differences in, 346 .

Potash in, 112.

Grasses, seeds of, follow man, 121.

Silica in, 170.

Valued in Germany, 169.

Compost for, 118.

Grauwacke, soil from, 147.

Growth of plants, conditions for the, 144 .

Guano, 95, 199.

Gypsum, decomposition of, 100 , 280.

Its influence, 101.

Use of, 191.

Theory of, 280.

Substitutes for, 282.

Action of, 247, 280.

Replaced, 248.

HAILSTONES, 91.

Hay, carbon in, 38.

Contains nitrogen, 176 . silica, 155.

Analysis of, 38 .

Haystack, effect of lightning upon a, 155.

Hesse, custom in, 213.

Hessian and English weights and measures, 416.
Hessian acre, 36.

Hibernating animals, 134.

Hippuric ucid, 97.

Horse, urine of the, 102

Concretions in the, 157 .

Horse-dung, action of water upon, 177.

Analysis of, 178 .

Human feces, analysis of, 179 .

Humate of lime, quantity received by plants, 37 .

Humic acid, 31, 65, 90.

Action of, 129 .

Properties of, 34 .

Is not contained in soils, 90 .

Quantity received by plants, 37 .

Insolubility of, 128.

Humus, 30, 90.

Action of, 63.

Analysis of, 32 .

Erroneous' opinions concerning, 49.

Extract of, 31.

Action upon oxygen, 127.

Coal of, 12!).

Conversion of woody fibre into, 358.

How produced, 358 .

Its insolubility, 127.

Properties of, 34 .

Replaced by charcoal, 78.

Source of carbonic acid, 65.

Theory of its action, 65 .

Unnecessary for plants, $33,77$.

Hungary, soils of, 240.

Hydrates, 31.

Hydrocyanic acid, 70, 290.

Hydrogen, assimilation of, 80-82.

Properties of, 25.

Excess of in wood accounted for, 81.

Of decayed wood, 359.

In plants, 263.

Of plants, source of, 82, 263.

Peroxide of, 294.

Hyett, $\boldsymbol{M r}$., on nitrate of soda, 206 , 271.

ICE. bubbles of gas in, 54 .

Indian corn, analysis of, 98.

Indifferent substances, 27 .

Infiammable air, 25.

Ingenhouss, his experiments, 49.

Inorganic compounds, 301 .

Action of, 374 .

In what they differ from organic, 302. 
Inorganic constituents of plants,|Manure, components of, should be $105,126$.

Compounds, stability of, 301 .

Iodine, 126.

Iron, oxide of, attracts ammonia, 103.

Irrigution of meadows, effect of, 127,169 .

Itch insect, 122.

JACKSON, analysis of horse-dung, 177.

On peat compost, 258.

Java, soil of, 244.

Juices of vegetables, 27.

LACTIC ACID, production of, 321 . Lava, soil from, 149.

Lead, salts of, compounds with organic matter, 383.

Leaves, absorb carbonic acid, 43.

Ashes of, contain alkalies, 154 .

Cessation of their functions, 68.

Change color from absorption of oxygen, 68.

Consequence of the production of their green principle, 173.

Decompose carbonic acid, 142.

Their office, 135.

Power of absorbing nutriment, how increased, 67 .

Quantity of carbon received by, 45.

Contain azotized matter, 188.

Lentils, analysis of, 159.

Life, notion of, 392 .

Light, absence of, its effect, 49 .

Chemical effects of, 105, 142.

Influences decomposition of carbonic acid, 53 .

Lime, phosphate of, 183, 184, 212.

Limestone, analysis of, 153 .

Lixiviation, 182.

Lucern, phosphate of lime in, 159.

Benefits attending its culture, 172.

Lucas, his experiments, 249.

MACAIRE-PRINCEP, his experiments, $164,256$.

Magnesia, phosphate of, in seeds, 62.

Maine, analysis of soil of, 246 .

Mannite, 139.

Manure, 174, 208.

Animal, yields ammonia, 95, 278 .

Artificial, 204, 212, 237.

known, 144.

Carbonic acid from, 99.

Human, 284.

Of the Chinese, 193.

Effect of, 173.

Bone, 183.

Daniell's artificial, 237.

Manuring of vines, 253,254 .

Maple juice, ammonia from, 94 .

Trees, sugar of, 94.

Meadons, irrigation of, 127, 169.

Medicine, action of, remedies in, 186.

Meconic acid, 115.

Melam, 70.

Melamin, 70.

Melitic acid, 363.

Mellon, 70 .

Merrimack Manuf. Co., first use of phosphate of soda by, 286 .

Metallic compounds required by plants, 60 .

Metamorphosis, 291.

Miasm, defined, 407.

Michaels, St., carbonic acid at, 79.

Minerals attract ammonia, 103.

Morbid poisons, 389.

Motion, its influence on chemical forces, 296.

Mould, vegetable, 363.

Conversion of woody fibre into, 364.

Condenses ammonia, 104.

Mouldering of bodies, 365 .

Must, fermentation of, 340 .

N.APLES, soils of, 152, 285.

Night-soil, 193, 199, 259, 234.

Nile, soil of its vicinity, 168.

Nitrate of soda as a manure, 206.

Theory of its formation, 277.

Of Peru, 270, 277.

Experiments with, 271.

Nitrated wheat, 272.

Flour, 275.

Nitric acid from ammonia, 336 .

- A nimals, 88.

How formed, 335.

Nitrification; 334 .

Condition for, 336.

Nitrogen from animals, 87.

Absorption of by plants, 267 .

Account of, 2.5

Application of substances con-

taining it, 99.

Assimilation of, 85, 97 . 
Nitrogen, chloride of, 293.

Characteristic of, 25 .

Compounds of, $25,27$. 319.

In albumen, 27.

From the atmosphere, 88.

In plants, 25, 27, 265 .

Source of, 28:3, 285 .

Production of, the object of agriculture, 99.

Transformation of bodies containing, 305. containing, 305.

In rice, 98 .

In solid excrements, 189.

In urine, 189.

Nutrition, conditions essential to, 22,59 .

Inorganic substances required in, 60 .

Superfluous, how employed, 67.

Of young plants, 172 .

OAKS, ashes of, 154 .

Excretions of, 49.

Dwarf, 66.

Followed by firs, 164 .

Oak-zoood affords humic acid, 35.

Composition of, 358.

Mouldered, analysis of, 359 .

Odor of substances, 345 .

Of gaseous contagious matter, 408.

Enanthic ether, 344.

Ohio, analysis of soils of, 245 .

Orcin, 325 .

Organs of excretion, 72.

Organic acids, 26.

Decomposition of, 295.

Chemistry, 21, 22.

Compounds, 82.

Compared with inorganic salts in plants, 301.

Orgunized bodies do not generate substances, 68 .

Osmazome, 317.

Oxalic acid, 70.

Oxford, experiments at, 257.

Oxamide, decomposition of, 391.

Oxides, metallic, in fir-wood, 111.

Oxygen, 26.

Äction on alcohol, 327.

Properties of, 26.

Absorption of, at night, 51
Oxygen, absorption by respiration, 72.

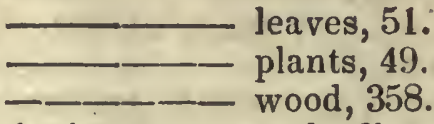

Action upon woody fibre, 359.

Its action in decomposition, 331.

Emitted by leaves, 43.

Given to air by land, 80 .

Extracted from air by mould, 364 .

In air, 28.

Consumption of, 40, 41 .

In water, 82.

Promotes decay, 130.

Separated during the formation of acids, 83.

Is furnished by the decomposition of water, 81 .

PAYEN, his table of composition of woods, 264.

Peat, compost of, 118, 258.

Analysis of, 185.

Perennial plants, how nourished, 135.

Peroxide, what, 295.

Peroxide of hydrogen, 294.

Peterson and Schodler; their analysis of woods, 52.

Phosphates necessary to plants, 155 .

Phosphate of iron, the probable cause of rust, 221.

In pollen, 182.

Phosphate of lime in teak wood, 156.

In forest soils, 182.

Phosphoric acid in ashes of plants, 155.

Phthisis, remedies in, 73.

Physiologists, their experiments not satisfactory, 62 .

Neglect of chemistry by, 56 .

Pipe-clay, ammonia in, 103.

Plants absorb oxygen, 50.

A shes of, salts in, 110.

Conditions necessary for their life, 62 .

Constituents of, 24.

Decay of, a source of oxygen, 84 .

Decompose carbonic acid, 43.

Development of, requisites for, 27, 117, 136, 143.

Effect of, on rocks, 150.

Elements of, 24.

Emit acetic acid, 150.

Exhalation of carbonic acid from, 53 . 
Plants, of a former world, 76 .

Formation of their components, 83.

Functions of, 44.

Improve the air, 47.

Influence of gases on, 50 .

- of shade, 50 .

Inorganic constituents of, 105 .

Life of, connected with that of animals, 22.

Milky-juiced, in barren soils, 78.

Nutritive qualities of, dependence on nitrogen, 265.

Organic acids in, 26, 106.

- salts in, 108.

Perennial, nourished, 135.

Products of, vary, 139.

Size of, proportioned to organs of nourishment, 66 .

Sources of their nourishment, 27.

Succession of, its advantage, 162.

Vital processes of, 84 .

Wild, obtain nitrogen from the air, 99.

Yield oxygen, 48.

Platinum does not decompose nitric acid, 292.

Ploughing, its use, 130.

Recornmended by Cato, 270 .

Poisons generated by disease, 374 .

Inorganic, 374-379.

Peculiar class of, 384 .

Rendered inert by heat, 389 .

Poisoning, superficial, 379 .

By sausages, 387.

Pompeii, air from, 41.

Bones from, 158.

Potush, action of, upon mould, 364 .

In limestones, 153.

In grapes, 112.

-Ley of, its effects on excrements, 99.

Presence of, in plants, accounted for, 148.

Replaced by soda, 113.

Required by plants, 62 .

Quantity in soils, 148.

Silicate of, in soils, 62 .

Sources of, 148.

Potatocs, oil of, 341.

Effect of, as food, 139.

Analysis of, 114.

Germination of, 133.

Produce of, increased, 134.

Poudrette, 199.

Products of transformations, 69.
Prince, $J$. $D$., first to apply phosphate of soda, \&c., 286.

Purgative effect of salts explained, 377.

Pus, globules in, 397.

Pusey, Mr., on nitrate of soda, 206.

Putrefaction, 63, 299, 300.

Of animals, 174.

Causes of, 292.

Communicated, 389.

Source of ammonia, 104.

- carbonic acid, 99.

Putrefying sausages, death from, 387 ; their mode of action, 388 . Substances, their effect on wounds, 389 .

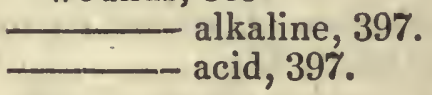

RADICAL, what, 69.

Rain-water, alkali extracted by, 150 .

Reduction of orides, 294.

Reeds and canes require silica, 155 .

Removal of branches, effects of, 132.

Reservoirs of dung, 191.

Respiration, oxygen consumed by, 41.

Rhine, soils in its vicinity, 168.

Wines, 342.

Rice, analysis of, 98 .

Ripening of fruit, 132.

Root secretions, 163, 256

Roots absorb, 107.

Emit excrementitious matter, 163.

Their office, 125.

Secretions of, 256 .

Rotation of crops, 161, 174.

SAL AMMONIAC, as manure, 282.

Saliculite of potash, 326.

Saline plants, 121.

Salsola kali, 113.

Salt, volatilization of, 123 .

Salts, absorption of, 116.

Effect of, on the organism, 375.

on flesh, 377.

on the stomach, 377 .

Organic, in plants, 27.

- in the blood, 376.

Passage of through the lungs, 376.

Salt-works, loss in, 124.

Saltroort, 122.

Sand, plants in, 78 .

Sandy soil, decay of wood in, 361 .

Situration, capacity of, 106. 
Sausages, poisonous, 387.

Saussure, his experiments on air, 42. Analysis of pines, 110.

On the growth of plants, 158.

Schubler, his observations on rain, 90.

Sea-vouter, analysis of, 124.

Contains carbon, 45.

Contains ammonia, 125.

Secretions, root, 256.

Silica, 170, in grasses, 155.

Solution of, 170.

In reeds and canes, 155.

Silicrte of potash in plants, 62.

As a manure, 187, 212, 213.

Siliceous sinter, 170.

Silver, carbonate of, action on organic acids, 295.

Salts, poisonous effects of, 382 .

Simple bodies, 21.

Sinapis alba, 410.

Size of plants proportional to organs of nourishment, 66 .

Smell, what, 345.

Snow-zoater, ammonia in, 91.

Soda may replace potash, 113.

Nitrate of, theory of its formation, $27 \%$.

Phosphate of, in calico printing, 286.

Soda-ash, 207.

Soils, advantage of loosening, 65 , 130.

Chemical constituents of, 208.

Best for meadow-land, 118.

Carbon restored to, 75.

Chemical nature of its influence, 167.

Constituents of, 208.

Exhaustion of, 151.

Ferruginous, improved, 130.

Fertile, contain phosphoric acid, potash, \&c., 242, 243.

Fertile, of Vesuvius, 149.

From lava, 149.

Of heaths, 223.

Imbibe ammonia, 99 .

Improved by crops, 161.

Impoverished by crops, 161.

Various kinds of, 208.

Stagnant water, effect of, 130.

Stalactites in caverns, 127.

Starch, 56; composition of, 83.

Accumulation of, in plants, 132.

Development of plants influenced by, 134.

Effect of, on malt, 74 .
Starch, vesicles in, 56

Product of the life of plants, 49.

In willows, 133.

Staunton, Sir G., on Chinese manure, 194.

Straw, analysis of, 38 .

Struve, experiments of, 151.

Substitution of bases, 109.

Subsoil ploughing, 215, 269.

Succession of crops, 275.

Succinic acid, 363.

Sugar, action of alkalies upon, 303.

Cocids upon, 303.

Composition of, 313.

Carbon in sugar, 38.

Contained in the maple-tree, 93.

In clerodendron fragrans, \&c., 138.

Devolopment of plants, influence on; 134.

Fermentation of, 313.

Formic acid from, 86.

In beet-roots, 93 .

Metamorphosis of, 313.

Organic compounds, all form sugar, 302.

Product of the life of plants, 49 .

Transformation of, 304 .

When produced, 67.

Sulphur, crystallized, dimorphous, 297.

In plants, 214 .

Sulphuric Acid, action of, on soils, 208, 248.

Sulphurous Acid arrests decay, 360 .

Swamp muck, 185.

Sweden, soils of, 243.

Swine, urine of, 202.

Synaptas, 411.

TABASHEER, 171.

Tables, of Hessian and English weights, 416.

Tannic Acid, 83.

Tartaric Acid, 83.

Converted into sugar, 83.

In wine, 342 .

Teak Tree, salts found in, 155.

Teeth, analysis of, 158.

Teltozo Parsnep, 66, 140.

Thenard, his experiments on yeast, 313.

Thermometers, scales of, 418 .

Tin, action on nitric acid, 292.

Tobacco, juice contains ammonia, 94.

Leaves of, 345 . 
Tobacco, value of, proportional to quantity of potash in the soil, 215.

Nitric acid in, 97.

In Virginia, 151.

Transformation, by heat, 306.

Chemical, 71, 2y1.

Chemical transformations differ from decompositions, 71 .

Of acetic acid, $3(16$.

Of arragonite, 298.

Of carbonic acid, 142.

Of meconic acid, 306.

Not affected by the vital principle, 74.

Explained, 74.

Of bodies containing nitrogen, 305.

Of boaiies destitute of nitrogen, 305.

Results of, 75.

Of sugar, 303 .

Of wood, 306 .

Of cyanic acid, 311 .

Of cyanogen, 311 .

Of gluten, 339 .

Transplantation, effect of, 132.

Trees, diseases of, 137.

Require alkalies, 154.

ULMIN, 30.

Urea, 70, 87; converted into carbonate of ammonia, 97 .

In urine, 189.

Uric Acid, yields ammonia, 192.

Transformations of, 193.

Urinary calculi, treatment of, 74.

Organs, eliminate nitrogen, 73.

Urine, contains nitrogen, 97.

Its use as a manure, 95, 201, 211.

Of men, \&c., 190.

Of horses, 202.

Human, analysis of, 190.

Of cows, 202 .

Its use in China and Flanders, $95,194$.

Of swine, 202.

$V A C C I N A T I O N$, its effect, 405.

Vegetable Albumen, 96.

Life, one end of, 23.

Mould, 36:3.

Juices, fermentation of, 314 .

Vesuvius, fertile soil of, 149.

Vines, new mode of manuring, 253. Juice of, yields ammonia, 94 .

Vinous Fermentution, 338.
Virginia, early products of its soils, 151.

Virus, of small pox, 405 .

Vaccine, 405.

Vitality, what, 59.

Vital Principle, 73.

Value of the term, 75.

How balanced in the blood, 374 .

Vital Processes of plants, 166.

Voelckel, his analysis of guano, 96 .

WATER, carbonic acid of, absorbed, 44.

Composition of, 81 .

Dissolves mould, 364 .

Freezing of, 296.

Plants, their action upon, 56.

Rain, contains ammonia, 91 .

— required by plants, 28 .

- required by gypsum, 102.

Hard, made soft, 92.

Salt, analysis of, 124.

Wavellite, 156.

West Indies, soil of, 244.

Wheat, analysis of, 154.

Ashes of, used as a manure, 213.

Exhausts, 152.

Nitrated, 272.

Gluten of, 94.

Manure for, 213.

Why it does not thrive on certain soils, 153.

In Virginia, 151.

Willrand, Dr., on maple sugar, 93. Willows, growth of, 133.

Wine, effect of gluten upon, 347.

Fermentation of. 347 .

Properties of, 347.

Substances in, 341 .

Taste and smell, 342.

Varieties of, 342.

Woad, decomposition of, 320 .

Wöhler, his analysis of limestone, 153.

Wood charcoal, may replace humus, 78.

Absorbs ammonia, 104.

Analysis of, 52.

Bread from, 133.

Composition of, 264.

Conversion of, into humus, 335.

Decay of, 357.

Requires air, 358.

Decomposition of, 320 .

Elements of, $358,360$. 
Wood, transformation of, 306.

Effect of moisture and air on, 358.

Formation of, 138.

Source of its carbon, 39.

Wood Coal, how produced, 365.

Analysis of, 367, 368.

Woody Fibre, changes in, 358.

Composition of, 358 .

Decomposition of, 358.

Formation of, 48.

Moist, evolves carbonic acid, 358.

Mould from, 364.

Wormwood, effect of its culture, 120.
Wort, fermentation of, 350 .

Wounds, effect of putrefying substances on, 386.

YEAST, 315.

Destroyed, 341.

Experiments on, 316.

Formed, 340.

Its mode of action, 318 .

Its production, 315 .

Two kinds of, 350 .

ZEINE, 98.

Zinc, decomposition of water with, 84. 
$+$
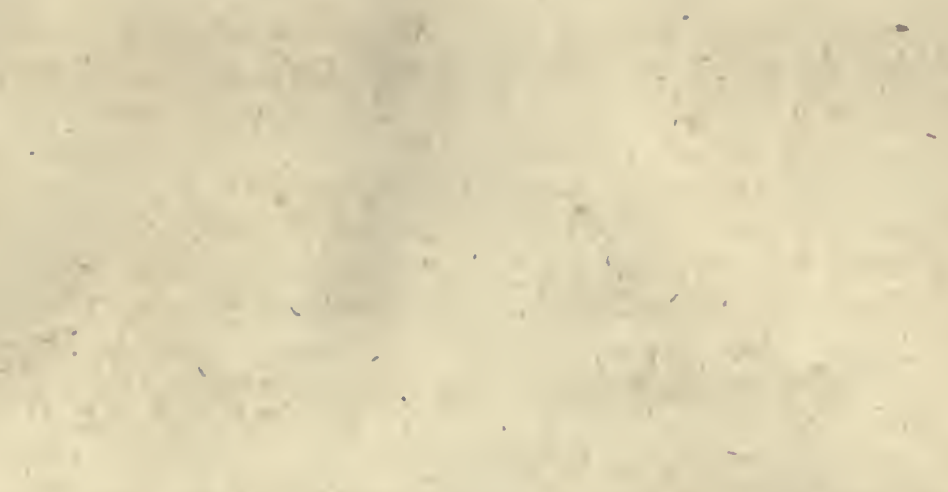

$\cdots$

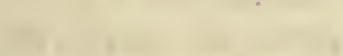
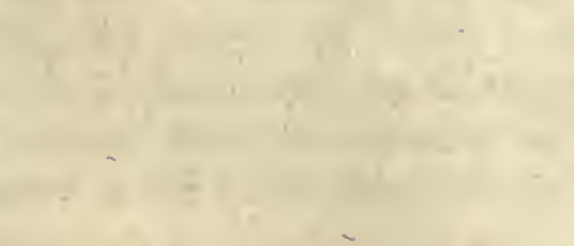

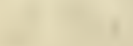
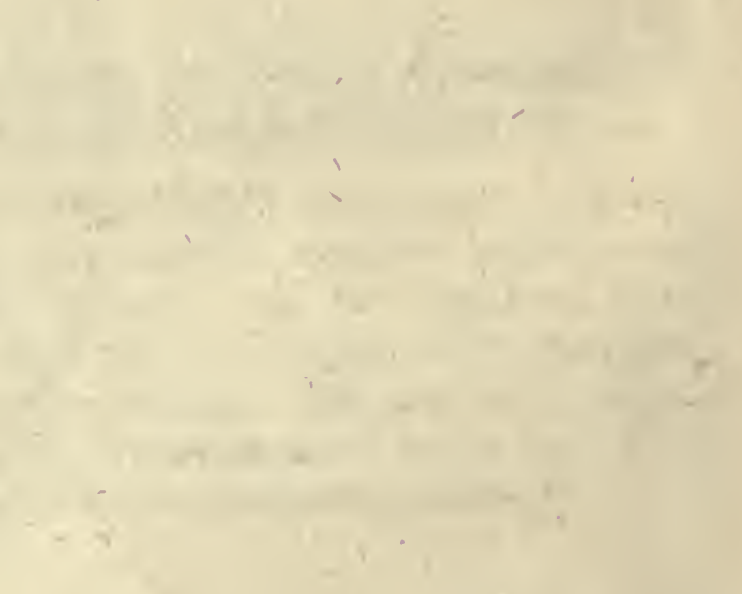

REPORT ON ORGANIC CHEMISTRY.

\section{NOTICES OF PART I.}

A GRICULTURAL CHEMISTRY.

This work has already acquired great reputation in Great Britain, and several notices and reviews of it have appeared in the foreign journals, all of which unite in expressing their high estimation of its contents. Three lectures have been recently delivered on Agriculture at Oxford by Dr. Daubeny, the distinguished Professor of Chemistry and Botany, in which he has illustrated and adopted Professor Liebig's views.

"Every page contains a mass of information. I would earnestly advise all practical men, and all interested in cultivation, to have recourse to the book itself. The subject is vastly important, and we cannot estimate how much may be added to the produce of our fields by proceeding on correct principles." - Loudon's Gardener's Magazine for March, 1841.

In alluding to this work, before the British Association for the Advancement of Science, Dr. Gregory remarked; -

"Every thing was simply and clearly explained. It was the first attempt to apply the newly created science of Organic Chemistry to Agriculture. In his opinion, from this day might be dated a new era in the art, from the principles established by Professor Liebig. He was of opinion, that the British Association had just reason to be proud of such a work, as originating in their recommendation."

The following is from the address at the Anniversary Meeting of the Royal Society, November 30, 1840, when one of the Copley medals was awarded to Professor Liebig, in presenting which, the President, the Marquis of Northampton, thus addressed Professor Daniell, who, in the absence of Professor Liebig, received for him the medal; - 
"I hold in my hand and deliver to you one of the Copley medals, which has been awarded by us to Professor Liebig. My principal difficulty, in the present exercise of this, the most agreeable part of my official duty, is to know, whether to consider M. Liebig's inquiries as most important in a chemical or in a physiological light; however that may be, he has a double claim on the scientific world, enhanced by the practical and useful ends to which he has turned his discoveries."

"It is the best book," writes. Mr. Nuttall, "ever published on Vegetable Chemistry as applied' to Agriculture, and calculated undoubtedly to produce a new era in the science."

Extract from a letter from Mr. Colman, Commissioner for the Agricultural Survey of Massachusetts, dated February 15 th, 1841 ; -

"It is the most valuable contribution to Agricultural science, which has come within my knowledge. It takes new views on many subjects, which have been long discussed without any progress towards determinate conclusions; and reveals principles, which are of the highest importance. Some of these principles require further elucidation and proof; but, in general, they are so well established by facts within my own observation, that in my opinion the truth, if not already reached, is not far distant."

From Silliman's Journal, January, 1841 ; -

"It is not too much to say, that the publication of Professor Liebig's Organic Chemistry of Agriculture, constitutes an era of great importance in the history of Agricultural science. Its acceptance as a standard is unavoidable, for, following closely in the straight path of inductive philosophy, the conclusions which are draun from its data are incontrovertible." - "To some, the style of this work may seem somewhat obscure ; but it will be found, on a re-perusal, that great condensation, brevity, and terseness, have been mistaken for obscurity." - "We can truly say, that we have never risen from the perusal of a book with a more thorough conviction of the profound knowledge, extensive reading, and practical research of its author, and of the invincible power and importance of its reasonings and conclusions, than we have gained from the present volume." 
In the notice from which the foregoing is extracted, the learned editors enumerate among the most important chapters, those on manure, the composition of animal manure, the essential elements of manure, bone manure, the supply of nitrogen by animal matter, mode of applying urine, value of human excrements, \&c.

The Second Part of the work is a masterpiece of condensed reasoning on chemical transformations, fermentation, decay, and putrefaction, and on contagion, poisons, and miasms.

From the Farmer's Register, Petersburg, Va., August, 1841 ; -

"This work of Professor Liebig has received more respectful attention and applause, than any on Agriculture that has issued from the press." - "No work have we yet seen that furnished to Agriculturists a more abundant store of scientific facts." - "We earnestly recommend to scientific Agriculturists and to Chemists to study Liebig."

"By the perusal of such works as this, the farmer need no longer be groping in the dark, and liable to mistakes; nor would the not unnatural odium of farming by the book, be longer existent.

"In conclusion, we recommend the work to the Agriculturist and to the Fiorticulturist, to the amateur florist, and to the curious student into the mysteries of organic life, - assured that they will find matter of interest and of profit in their several tastes and pursuits."- Hovey's Magazine of Horticulture, \&c., September, 1841.

"We regard the work of Liebig as a work of extraordinary philosophical acumen, and conferring upon him the highest honor. The more it is examined, the deeper will be the interest which it will create, and the stronger the admiration of the ability with which it is written. It is not a work to be read, but studied; and if further inquiries and experiments should demonstrate, as seems to us from many facts within our own knowledge in the highest degree probable, the soundness of his views, his work, not merely as a matter of the most interesting philosophical inquiry, but of the highest practical utility, will be invaluable." - North American Review, July, 1841. 
"Dr. Webster has rendered an important service to the agricultural community, by presenting an edition of this now well known and highly esteemed work. Professor Liebig has for some time been known as one of the most eminent chemists of Europe, and the publication of this work in England has excited general and unqualified approbation. Almost all the scientific and literary periodicals have been loud in its praise, and all concur in the opinion, that a new era in agriculture must date from its appearance. The present edition has been greatly increased in value and utility by the additions which it has received from the American editor. 'The Notes and Appendix contain much important information for the agriculturist, and the explanations which have been added of chemical terms, render it intelligible to all. It should be in the hands of every farmer. The typography and general appearance of the volume is such as might be expected from the University Press." - Christian Examiner, July, 1841.

"In the present work, Dr. L. has pointed out the path to be pursied, and has amply vindicated the claim of science to be considered the best guide, by correcting the erroneous views hitherto prevailing, of the sources whence plants derive their nourishment, by developing the true causes of fertility in soils, and finally, by establishing, on a firm basis, the true doctrine of manures." - Quarterly Review, March, 1842.

NOTICE OF PART II.

\section{A N I M A L C H E I S T R Y.}

"While we have given but a very imperfect sketch of this original ard profound work, we have endeavored to convey to the reader some notion of the rich store of interesting matter which it contains. The chemist, the plyssiologist, the medical man, and the agriculturist, will all find in this volume many new ideas and many useful practical remarks. It is the first specimen of what modern organic chemistry is capable of doing for physiology; and we have no doubt that, from its appearance, physiology will date a new era in her advance. We have reason to know that the work, when in progress, at all events the more important parts of it, were submitted to Müller of Berlin, Tiedemann of Heidelberg, and Wagner of Göttingen, the most distinguished physiologists of Germany; and without inferring that these gentlemen are in any way pledged to the author's opinions, we may confidently state that there is but one feeling among them as to the vast importance of Chemistry to Physiology at the present period; and that they are much gratified to see the subject in such able hands." - Quarterly Review. 


\title{
THE
}

\section{HISTORY OF HARVARD UNIVERSITY.}

\author{
By JOSIAH QUINCY, LL. D.,
}

PRESIDENT OF THE UNIVERSITY.

CAMBRIDGE :

PUBLISHED BY JOHN OWEN.

[Royal 8vo. Vols. I. and II. pp. 612 and 728.]

21 Engravings.

"This History is a monument of patient and unwearied investigation, - of rigid impartiality and discrimination in deductions from time-worn records. It embraces the events of two centuries, and historical and biographical notices of nearly every individual whose name is found connected with any important incident in the annals of the University." Boslon Courier.

"There is no hazard in saying, that this work is rich in materials, many of which have escaped the notice of even extensive readers, and that it bears marks of thorough research, and great care in the collection and verification of facts, and judgment and skill in the arrangement and developement of the narrative." - Daily Advertiser.

"The American press has rarely, if ever before, sent forth two such beautiful volumes in typographical execution, as these, containing an admirable and interesting history of the venerable University of Cambridge. To the numerous Alumni of Harvard, these volumes will be precious indeed." - New York American.

"The history of the University is now written; and it needs no prophetic sagacity or boldness to assert, that it will 
endure. For the indefatigable diligence and learned research with which the materials have been assembled; for the fullness, candor, and impartiality, with which they are now exhibited; for the light reflected thus on the history, not only of the College, but of the times; in fine, for what he has here done to establish the claims of Harvard College, in the successive periods of its history, to the gratitude and veneration of her sons in all coming time,.-we offer him, in their name, nor will they deem it presumptuous, our cordial thanks." - Christian Examiner.

"We expected to find in these volumes the authentic results of diligent research, and accordingly, a valuable contribution to the completeness of existing aids to an acquaintance with the men and doings of the ancient times. But we confess we did not expect to find them so fruitful in entertainment, and in materials for engaging and profitable, as well as (to a patriot) complacent reflection. We did not expect to see a record of the fortunes of a single institution of learning, taking the place, which this seems to us destined to take, arnong works of historical literature.

"This is not a book to be welcomed and enjoyed by the friends of Harvard College alone, nor by either of the small classes of New England, or of academical antiquaries, but one which will sustain permanent claims on the attention of the general student of history." - North American Review.

This work is, in fact, not simply the history of one of our most ancient literary institutions, but a history of the progress of letters in New England from the earliest days of the Puritan colonists ; the history of the most illustrious minds, for heroism and genius, which have adorned the annals of Massachusetts for the last two centuries.

The whole net proceeds of the sale of these volumes will be devoted to assist indigent students. 


\section{WORKS RECENTLY PUBLISHED BY}

\section{J. OWEN, CAMBRIDGE.}

THE HISTORY OF HARVARD UNIVERSITY, by JOSIAH Quincr, LL. D., President of the University. With 21 Engravings. 2 vols. royal $8 \mathrm{vo}$. cloth.

VOICES OF THE NIGHT, by HENRY WADSWORTH LONGFELLOW. 6th edition. 16mo. boards.

THE SAME. royal 8vo. fine paper. boards.

THE CLOUDS OF ARISTOPHANES. With Notes by C. C. FeLton, Professor of Greek Literature in Harvard University. 12mo. cloth.

LECTURES ON MODERN HISTORY, from the Irruption of the Northern Nations to the close of the American Revolution. By William Sмiтth, Professor of Modern History in the University of Cambridge. From the Second London Edition, with a Preface, List of Books on American History, \&c., by JARED Sparks, LL. D., Professor of Ancient and Modern History in Harvard University. 2 vols. 8vo. cloth.

BALLADS AND OTHER POEMS, by HENRY WADSWORTH LONGFELLow. 4th edition. $16 \mathrm{mo}$. boards.

THE SAME. royal 8vo. fine paper. boards.

A NARRATIVE OF-VOYAGES and Commercial Enterprises, by R. J. Cleveland. 2 vols. $12 \mathrm{mo}$. cloth.

AN-INQUIRY into the Foundation, Evidences, and Truths of Religion. By Henry Ware, D. D., late Hollis Professor of Divinity in Harvard College. 2 vols. 12mo. cloth.

HENRY OF OFTERDINGEN : A Romance. From the German of Novalis (Friedrich von Hardenberg). 12mo. cloth.

PROF. LIEBIG'S REPORT ON ORGANIC CHEMISTRY.

PART I.

\section{AGRICULTUR.AL CHEMISTRY.}

CHEMISTRY in its Application to Agriculture and Physiology. By Justus Liebig, M.D., Ph. D., F. R. S., M. R. I. A., Professor of Chemistry in the University of Giessen, \&c. Edited from the Manuscript of the Author, by Lyon Playfair, Ph. D. With numerous Additions, and a New Chapter on Soils. Third American from the Second English Edition, with Notes and Appendix, by John W. Webśter, M. D., Erving Professor of Chemistry in Harvard University. $12 \mathrm{mo}$. 
PART II.

\section{ANIMAL CHEMISTRY.}

ANIMAL CHEMISTRY, or Organic Chemistry in its Application to Physiology and Pathology. By Justus LieBig, M. D., Ph. D., F.R.S., M. R.I. A., Professor of Chemistry in the University of Giessen, \&c. Edited from the Author's Manuscript, by WiLliam Gregory, M. D., F.R. S. E., M.R.I. A., Professor of Medicine and Chemistry in the University and King's College, Aberdeen. With Additions, Notes, and Corrections, by Dr. Gregory, and others by John W. Webster, M.D., Erving Professor of Chemistry in Harvard University. 1 vol. $12 \mathrm{mo}$.

\section{N P R ES,}

A TREATISE ON MINERALOGY, on the Basis of Thomson's Outlines, with Numerous Additions; comprising the Description of all the new American and Foreign Minerals, their Localities, \&c. Designed as a Text-Book for Students, Travellers, and Persons attending Lectures on the Science. By J. W. WEBSTER, M. D., Professor of Chemistry and Mineralogy in Harvard University. 8vo.

THE EVIDENCES OF THE GENUINENESS OF THE GOSPELS. By Andrews Norton. Vols. II. and III. (being the completion of the work). 8 vo.

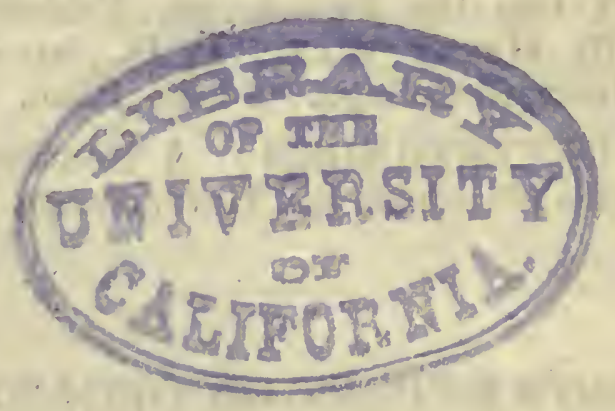





RETURN TO the circulation desk of any

University of California Library

or to the

NORTHERN REGIONAL LIBRARY FACILITY Bldg. 400, Richmond Field Station

University of California

Richmond, CA 94804-4698

ALL BOOKS MAY BE RECALLED AFTER 7 DA

2-month loans may be renewed by calling

510 (475) 642-6753

1-year loans may be recharged by bringing boc to NRLF

Renewals and recharges may be made 4 de prior to due date

DUE AS STAMPED BELOW

\section{MAR 121992}

FEB 171994

JAN 022001

OCT 2928984

Rec. JUN $21^{\circ} 05$ 


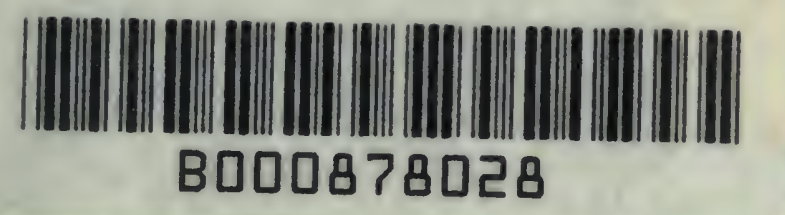

$5013 / 4$

The (ja) 3) H. 9.

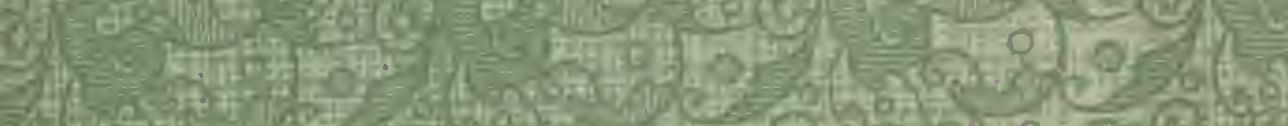
2.5.

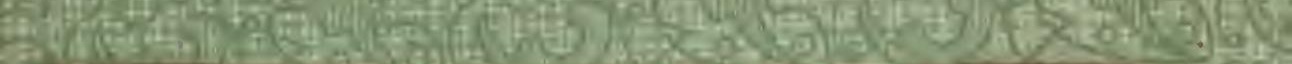

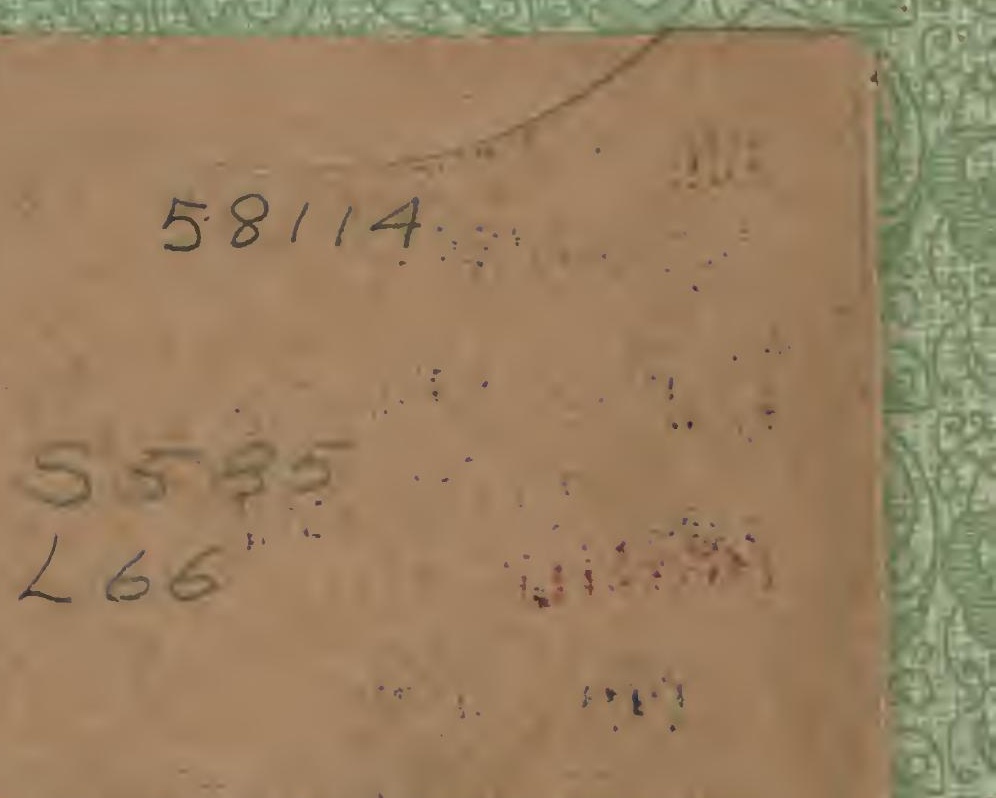

UNIVERSITY OF CALIFORNIA LIBRARY KA

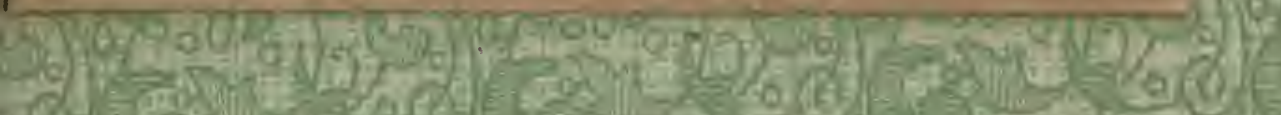
2.

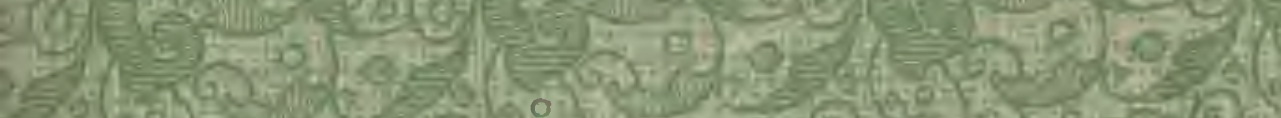

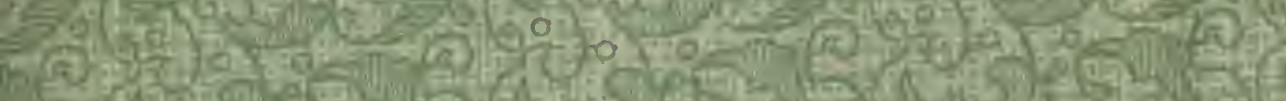
(5)

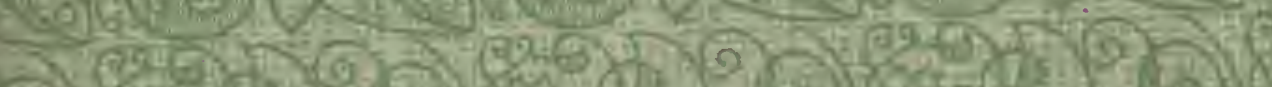

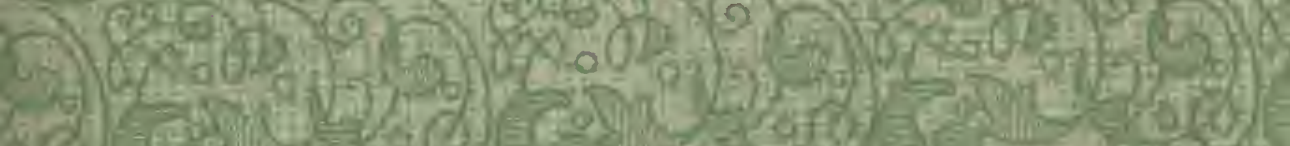
Q(2) I.

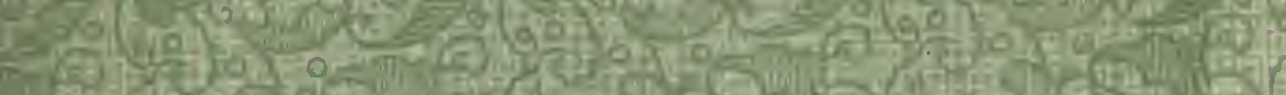
7. 5 en

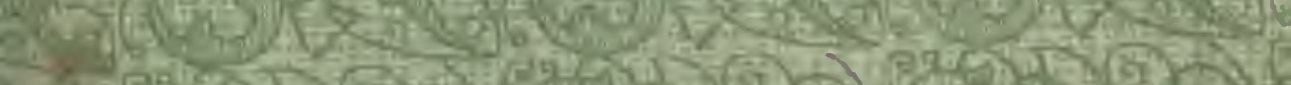

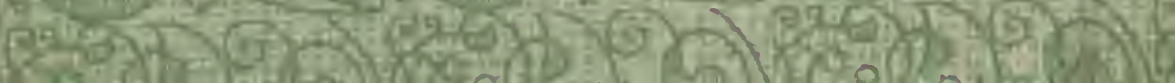


